Iron and Copper Active Sites in Zeolites and their Correlation to Metalloenzymes

Benjamin E. R. Snyder ${ }^{1}$, Max L. Bols ${ }^{2}$, Robert A. Schoonheydt ${ }^{2 *}$, Bert F. Sels, ${ }^{2 *}$ and Edward I. Solomon ${ }^{1,3 *}$

1 Department of Chemistry, Stanford University, Stanford, California 94305, USA.

2 Department of Microbial and Molecular Systems, Centre for Surface Chemistry and Catalysis, KU Leuven - University of Leuven, Celestijnenlaan 200F, B-3001 Leuven, Belgium.

3 Photon Science, SLAC National Accelerator Laboratory, 2575 Sand Hill Road, Menlo Park, California 94025, USA

Abstract. Metal-exchanged zeolites are a class of heterogeneous catalysts that perform important functions ranging from selective hydrocarbon oxidation to remediation of $\mathrm{NO}_{x}$ pollutants. Among these, copper and iron zeolites are remarkably reactive, hydroxylating methane and benzene selectively at low temperature to form methanol and phenol, respectively. In these systems, reactivity occurs at well-defined molecular transition metal active sites, and in this review we discuss recent advances in the spectroscopic characterization of these active sites and their reactive intermediates. Site-selective spectroscopy continues to play a key role, making it possible to focus on active sites that exist within a distribution of inactive spectator metal centers. The definition of the geometric and electronic structure of metallozeolites has advanced to the level of bioinorganic chemistry, enabling direct comparison of metallozeolite active sites to functionally analogous $\mathrm{Fe}$ and $\mathrm{Cu}$ sites in biology. We identify significant parallels and differences in the strategies used by each to achieve high reactivity, highlighting potentially interesting mechanisms to tune the performance of synthetic catalysts.

*E-mail: edward.solomon@stanford.edu, bert.sels@biw.kuleuven.be, robert.schoonheydt@biw.kuleuven.be 
Contents 2

1. Introduction 4

1.1. Challenges of Industrial Selective Partial Oxidation 5

1.1.1. Conversion of Methane to Methanol 6

1.1.2. Conversion of Benzene to Phenol 10

1.2. Enzymes as Industrial Oxidation Catalysts $\quad 12$

1.3. TMI Zeolites as Industrial Oxidation Catalysts $\quad 14$

2. Copper and Iron Zeolites 18

2.1. Zeolite Structure and Properties 18

2.2. TMI Exchange $\quad 25$

2.3. Activation and Autoreduction $\quad 27$

2.4. Factors Influencing Active Site Nuclearity 31

2.5. Coordination of Divalent Metal lons in Dehydrated Zeolites 36

3. Active Site Spectroscopic Probes 43

3.1. Ground and Excited State Properties of Transition Metal Sites 44

3.1.1. Paramagnetic Ground States $\quad 45$

3.1.2. Ligand Field Excited States $\quad 50$

3.1.3. Charge Transfer Excited States 52

3.2. Resonance Raman Spectroscopy 54

3.3. Magnetic Circular Dichroism Spectroscopy 56

3.3.1. $A$ - and $B$-term Mechanisms $\quad 58$

3.3.2. The $C$-term Mechanism $\quad 59$

3.3.3. Saturation Magnetization: Variable-Temperature 62

Variable-Field MCD

3.4. Other Spectroscopic Methods with Single Site Resolution 64

3.4.1. Electron Paramagnetic Resonance Spectroscopy 65

3.4.2. Mössbauer Spectroscopy 69

3.4.3. Nuclear Resonance Vibrational Spectroscopy 71

4. Cu/O Active Sites in Zeolites 73

4.1. Methane Hydroxylation by Cu-ZSM-5 73

4.2. Reactivity of $\left[\mathrm{Cu}_{2} \mathrm{O}\right]^{2+}$ Active Sites in Zeolites $\quad 78$

4.3. Activation of $\mathrm{O}_{2} \quad 82$

4.4. Activation of $\mathrm{N}_{2} \mathrm{O}$

4.5. Other $\mathrm{Cu}_{x} \mathrm{O}_{y}$ Active Sites of Methane Hydroxylation 90

4.5.1. Trinuclear $\left[\mathrm{Cu}_{3} \mathrm{O}_{3}\right]^{2+}$ Active Sites 92

4.5.2. Other $\mathrm{Cu}_{n} \mathrm{O}_{x}$ Copper Clusters, $\mathrm{n}>3$. 95

4.5.3. Comparative Reactivity of $\mathrm{Cu}_{n} \mathrm{O}_{x}$ Clusters 96

4.5.4. Mononuclear $[\mathrm{Cu}(\mathrm{OH})]^{+}$Active Sites 100

5. Fe/O Active Sites in Zeolites 102

5.1. Introduction 102

5.2. The $\alpha-\mathrm{Fe}(\mathrm{II})$ Active Site 103

5.2.1. $\alpha$-Fe(II) Structure 103

$\begin{array}{ll}\text { 5.2.2. } \alpha-\mathrm{Fe}(\mathrm{II}) \text { Spectroscopy } & 105\end{array}$ 
5.2.3. Reactivity of $\alpha-\mathrm{Fe}(\mathrm{II}) \quad 108$

5.3. The $\alpha$-O Reactive Intermediate 110

5.3.1. $\alpha$-O Structure 110

5.3.2. $\alpha$-O Spectroscopy 112

5.3.3. Reactivity of $\alpha-\mathrm{O} \quad 114$

5.4. Selective Hydrocarbon Oxidation with $\mathrm{H}_{2} \mathrm{O}_{2}$. 116

6. Correlation of Metallozeolites to Metalloenzymes 118

6.1. Cu-Zeolites and pMMO 119

6.2. Fe-Zeolites and sMMO 124

6.3. From Bioinorganic to Zeolite Catalysis: Tuning the Reactivity 129 of Metal Active Sites

6.3.1. Metal-Oxygen Covalency 129

6.3.2. Second Sphere Effects 131

6.3.3. The Entatic State 132

7. Concluding Comments 136

Author Information 138

Corresponding Authors 138

Notes 138

Acknowledgements 138

$\begin{array}{lr}\text { Abbreviations } & 139\end{array}$

$\begin{array}{ll}\text { References } & 140\end{array}$

Author Biographies 172 
1 - Introduction. Based on promising applications in the remediation of noxious NOx and $\mathrm{N}_{2} \mathrm{O}$ from combustion engines and the mild partial oxidation of unreactive hydrocarbons, research into redox catalysis for selective partial oxidation using copper and iron exchanged zeolites has moved forward at an appreciable pace. The general challenge associated with the latter application is the formation of highly reactive oxygen species, combined with the selectivity towards partial oxidation products over thermodynamically favoured full oxidation into $\mathrm{CO}_{2}$ and $\mathrm{H}_{2} \mathrm{O}$. In addition, the catalysts should preferably contain earth-abundant transition metals $(\mathrm{Fe}, \mathrm{Cu}, \mathrm{Co}, \mathrm{Ni}, \ldots)$ and make use of oxidants (e.g., $\mathrm{O}_{2}, \mathrm{~N}_{2} \mathrm{O}, \mathrm{NO}, \ldots$ ) which are cheap and benign, or which can be recovered from waste streams. The activation of the strong $(104 \mathrm{kcal} / \mathrm{mol})$ methane $\mathrm{C}-\mathrm{H}$ bond under mild conditions can be seen as a model reaction requiring such reactive oxygen species. The performance in the partial oxidation of methane under mild conditions will function as a benchmark for defining the zeolitic $\mathrm{Cu} / \mathrm{O}$ and $\mathrm{Fe} / \mathrm{O}$ species as active sites in this review. Another industrially relevant conversion, the partial oxidation of benzene to phenol, faces similar challenges and will also be treated.

Over the past years, previously reported active sites in $\mathrm{Cu}$ and $\mathrm{Fe}$ exchanged zeolites have been characterized in detail and, simultaneously, new observations have opened up novel tracks of research. Combinations of spectroscopic techniques and the use of site-selective spectroscopy complementing bulk techniques and reactivity studies as well as computational models have had a significant hand in this progress. Focus is put on the formation, stability and characterization of $\mathrm{Cu} / \mathrm{O}$ and $\mathrm{Fe} / \mathrm{O}$ reactive species in zeolites and on the relation between their structural properties and their exceptional 
reactivity for inert hydrocarbon oxidation. In section 2, a general introduction on zeolites is given and transition metal ions (TMI) in zeolites are discussed.

Parallels can be drawn between these zeolitic systems and metalloenzymes which catalyse similar reactions, both on the level of catalytic function and on the level of characterization. Characterization techniques which will be discussed in this review (section 3), including resonance Raman spectroscopy, variable-temperature-variablefield magnetic circular dichroism, and nuclear resonance vibrational spectroscopy, have recently crossed over from the bio-inorganic world to the field of heterogeneous catalysis. In sections 4 and 5 , these techniques are applied to reactive copper and iron active sites for methane activation. Spectroscopic data are coupled to electronic structure calculations, showing how the unique geometric and electronic structures of metallozeolite active sites lead to high reactivity. Finally, a comparative study of oxidation chemistry by metalloenzymes and metallozeolites is presented in section 6 . Structure-function relationships are well established for iron and copper active sites in biology, and here we extend concepts from bioinorganic chemistry to metallozeolite catalysis.

1.1 - Challenges of industrial selective partial oxidation. The partial oxidation of inert hydrocarbons, such as methane and benzene, presents inherent challenges for catalysis. High selectivity towards partially oxygenated products of inert hydrocarbons is hampered by their decreased $\mathrm{C}-\mathrm{H}$ bond strengths. This causes a preference for the formation of over oxidized products through consecutive oxygenation reactions, and imposes an upper limit to the achievable selectivity. Due to the high activation barriers 
common for these reactions, attaining high yields tends to require an extended contact time with the oxidation catalyst. This leads to a tradeoff between conversion, requiring long contact times, and selectivity, requiring short contact times. Workarounds to this issue involve the synthesis of stable, partially oxidized intermediates, which are converted to the desired products in subsequent steps. These intermediates include syngas and strongly adsorbed (coordinated) reactive species on the heterogeneous (homogeneous) catalyst surface (active site).

1.1.1 - Conversion of Methane to Methanol. To valorize methane for chemical synthesis, the notoriously inert $\mathrm{CH}_{4}$ molecule must first be functionalized. The difficulty of methane functionalization stems from the molecule's electronic structure, shown in figure 1. Due to the high strength of the methane $\mathrm{C}-\mathrm{H}$ bond, the occupied bonding levels $\left(1 a_{1}, 1 t_{2}\right)$ are significantly stabilized (figure $\left.1 A\right)$. This is evident from the high ionization potential of methane $(12.61 \mathrm{eV}) .{ }^{1}$ The high symmetry of methane also results in bonding molecular orbitals that are delocalized over many atoms (1 $\mathrm{a}_{1}$ and $1 \mathrm{t}_{2}$ in figure 1B). These are resistant to electrophilic attack due to poor energy match and overlap

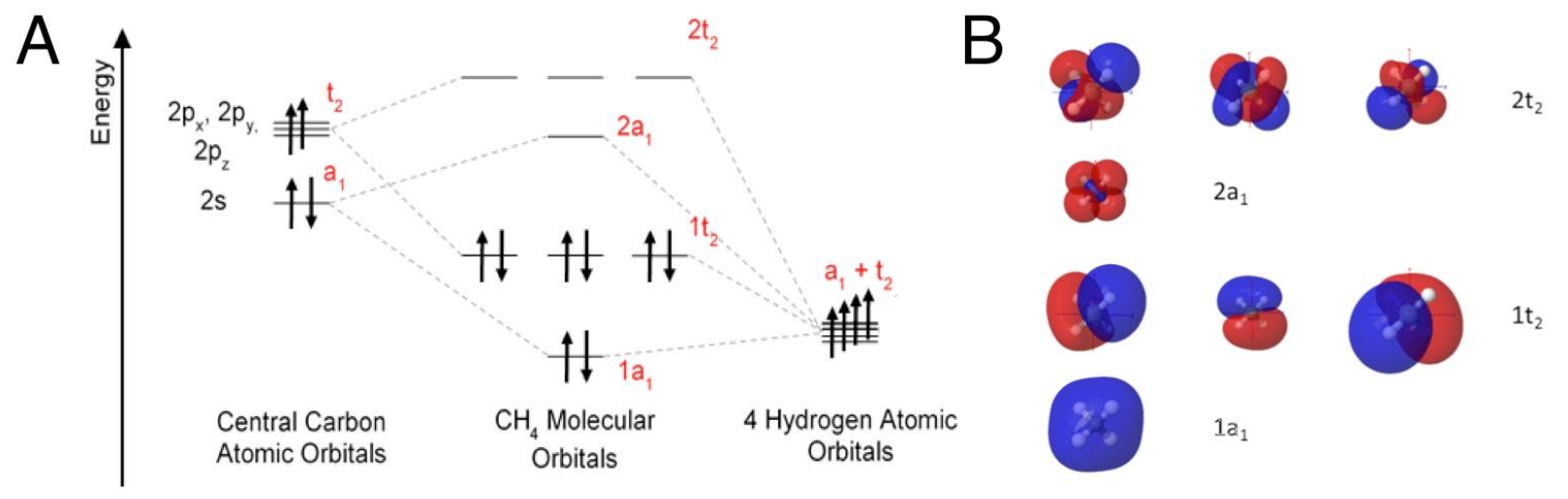

Figure 1. A) Molecular orbital diagram for methane derived from symmetry adapted linear combinations of carbon and hydrogen valence atomic orbitals. B) Molecular orbitals of methane, showing bonding ( $1 \mathrm{a}_{1}$, $\left.1 \mathrm{t}_{2}\right)$ and antibonding levels $\left(2 \mathrm{a}_{1}, 2 \mathrm{t}_{2}\right)$. 
with molecular orbitals of incoming electrophiles. The unoccupied antibonding levels $\left(2 \mathrm{a}_{1}, 2 \mathrm{t}_{2}\right.$ in figure 1$)$ are significantly destabilized and delocalized, and this makes methane similarly resistant to nucleophilic attack. Finally, methane is resistant to deprotonation $\left(\mathrm{pK}_{\mathrm{a}}=48\right)$ due to the high covalency of its $\mathrm{C}-\mathrm{H}$ bonds. ${ }^{2}$

Several strategies have been developed to tackle the problem of selective methane activation under mild conditions, and these can be divided into four classes shown in figure $2 .^{3}$ In oxidative addition (figure $2 \mathrm{i}$ ), a low-valent metal reductively cleaves the $\mathrm{C}-\mathrm{H}$ bond by donating two electrons into the $\mathrm{C}-\mathrm{H} \sigma^{*}$ antibonding orbital. In electrophilic activation (e.g. Shilov chemistry, figure 2ii), a C-H bonding orbital donates into a vacant metal $d$ orbital, and this weakens and polarizes the $\mathrm{C}-\mathrm{H}$ bond so that methane can be deprotonated. In sigma bond metathesis (figure 2iii), binding to the $\mathrm{M}$ $\mathrm{X}$ fragment plays a similar role in activating the $\mathrm{C}-\mathrm{H}$ bond. Finally, reactive metaloxygen species (figure 2iv) are highly electrophilic, with low-lying unoccupied molecular orbitals that are localized on a metal-oxygen fragment. These are activated as acceptor orbitals for $\mathrm{H}$-atom abstraction (HAA), even from the stable $\mathrm{C}-\mathrm{H}$ bonding levels of methane shown in figure 1 . This is the strategy employed by nature and metallozeolites to activate strong aliphatic $\mathrm{C}-\mathrm{H}$ bonds. (Activation of aromatic $\mathrm{C}-\mathrm{H}$ bonds proceeds through a different mechanism, discussed below.)

\begin{tabular}{|c|c|c|c|}
\hline \multicolumn{4}{|c|}{ Low Temperature $\mathrm{CH}_{4}$ activation with transition metals } \\
\hline (i) Oxidative Addition & (ii) Electrophilic Activation & (iii) $\sigma$-bond metathesis & (iv) Reactive O-species \\
\hline 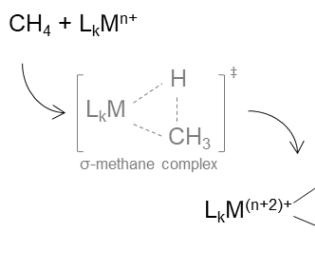 & 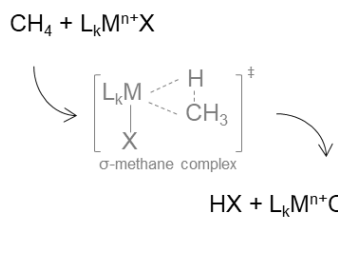 & 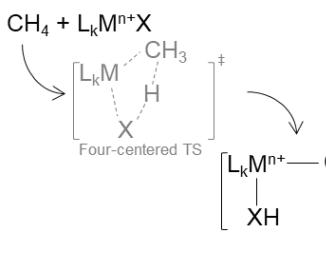 & $\longrightarrow \mathrm{L}_{k} \mathrm{M}^{(\mathrm{n}+2)+}=\mathrm{O}+\mathrm{D}$ \\
\hline
\end{tabular}

Figure 2. The four main mechanisms for $\mathrm{C}-\mathrm{H}$ bond activation in methane (i) oxidative addition, (ii) electrophilic activation, (iii) sigma-bond metathesis, and (iv) oxidation with a reactive oxygen species. 
Specific catalytic systems have been developed to selectively oxygenate methane while avoiding excessive oxidation to thermodynamically favored products (e.g. formate, $\mathrm{CO}_{2}$ ). Shilov systems operate through the electrophilic activation of the C$\mathrm{H}$ bond by a $\mathrm{Pt}^{\| \prime}$ complex. ${ }^{4,5}$ Stronger $\mathrm{C}-\mathrm{H}$ bonds have higher electron density than weaker $\mathrm{C}-\mathrm{H}$ bonds, and are therefore more susceptible to electrophilic activation, suppressing over-oxidation. Oxidation with soft electrophiles, such as sulfur, is also proposed. ${ }^{6}$ All have their limitations, however, for which we refer the reader to specific literature on the topic. ${ }^{4,7-9}$ The use of a reactive oxygen center for the $\mathrm{C}-\mathrm{H}$ bond activation shifts the challenge from the $\mathrm{C}-\mathrm{H}$ cleavage to the formation of isolated, highly reactive oxygen species. This requires the activation of an oxidant - ideally $\mathrm{O}_{2}$. Binding dioxygen to a reduced metal center results in activation at the oxy, superoxy, or peroxy level. With the participation of more than one reduced metal, the O-O bond can be cleaved resulting in activated metal-oxo species. The reaction of triplet $\mathrm{O}_{2}$ (oxy) species with singlet organic substrates is spin-forbidden and therefore slow. Superoxy, peroxy, and oxo species do not suffer from this limitation, however, thus reductive activation of $\mathrm{O}_{2}$ can overcome the spin-forbiddenness of its reaction with organic substrates. Reactive metal-oxygen species can also be generated in zeolites using nitrous oxide or hydrogen peroxide. This is particularly important in the chemistry of Fe-zeolites, where methane-reactive sites cannot be formed with $\mathrm{O}_{2}$. (Although we note copper zeolites are activated equivalently by both $\mathrm{N}_{2} \mathrm{O}$ and $\mathrm{O}_{2}$.) For $\mathrm{N}_{2} \mathrm{O}$, there is an entropic driving force associated with irreversible $\mathrm{N}_{2}$ release during $\mathrm{O}$-atom transfer, and this can be used to drive the generation of unstable (and therefore reactive) metal-oxo sites. However, the use of $\mathrm{N}_{2} \mathrm{O}$ as the final oxidant for methane activation inevitably implies a 
bottleneck for future upscaling to an industrially relevant scale. Industrially available side streams of $\mathrm{N}_{2} \mathrm{O}$ are currently dwarfed by the intended scale of methane upgrading. ${ }^{10}$ Alternatively, other oxidants can be used $\left(\mathrm{O}_{3}\right.$, tert-butyl-hydroperoxide, $\mathrm{HNO}_{3}, \mathrm{H}_{2} \mathrm{SO}_{4}$, $\mathrm{NaClO}, \mathrm{NaClO}_{2}, \mathrm{H}_{2} \mathrm{O}_{2} \ldots$...) but these cannot compete with freely available atmospheric $\mathrm{O}_{2}$. A strongly related challenge in catalysis is therefore the activation of molecular oxygen, either to form intermediary oxidants, or to directly perform the desired oxidation reaction in the next step.

One candidate platform chemical obtained from methane is methanol. Methanol is subsequently converted to a broad range of petrochemical products such as formaldehyde, dimethyl-ether and propylene, among others. Moreover, methanol is an easily transported liquid at room temperature. Methane transport, on the other hand, requires extensive infrastructure (liquefied natural gas (LNG) terminals, LNG carriers and natural gas pipeline systems) with a high risk of leakages contributing to the greenhouse effect. ${ }^{11}$ Currently implemented methane to methanol processes all pass over syngas as an intermediate, requiring large scale plants which operate at high pressures and temperatures $(\mathrm{ICl}$ process - figure 3 , top). ${ }^{12}$ For syngas production, three well established, heterogeneously catalyzed processes exist: 1) steam reforming over nickel catalysts, 2) autothermal reforming over nickel supported on magnesium aluminium spinel and 3) dry reforming over nickel or

\begin{tabular}{|c|c|}
\hline INDIRECT SYNGAS PATHWAY & \\
\hline 1) Steam Reforming: & \\
\hline $\mathrm{CH}_{4}(\mathrm{~g})+\mathrm{H}_{2} \mathrm{O}(\mathrm{g}) \rightarrow \mathrm{CO}(\mathrm{g})+3 \mathrm{H}_{2}(\mathrm{~g})$ & $\Delta \mathrm{H}_{\mathrm{r}}^{\circ}=206 \mathrm{~kJ} / \mathrm{mol}$ \\
\hline 2) Methanol Formation: & \\
\hline $\mathrm{CO}+2 \mathrm{H}_{2} \rightarrow \mathrm{CH}_{3} \mathrm{OH}$ & $\Delta \mathrm{H}_{\mathrm{r}}^{\circ}=-90.7 \mathrm{~kJ} / \mathrm{mol}$ \\
\hline DIRECT PARTIAL OXIDATION PATI & \\
\hline $\mathrm{CH}_{4}(\mathrm{~g})+1 / 2 \mathrm{O}_{2}(\mathrm{~g}) \rightarrow \mathrm{CH}_{3} \mathrm{OH}(\mathrm{g})$ & $\begin{aligned} \Delta \mathrm{H}^{\circ}{ }_{\mathrm{r}} & =-126.43 \mathrm{~kJ} / \mathrm{mol} \\
\Delta \mathrm{S}^{\circ}{ }_{\mathrm{r}} & =-49.3 \mathrm{~J} / \mathrm{mol} / \mathrm{K}\end{aligned}$ \\
\hline
\end{tabular}

Figure 3. Thermodynamics of methane-to-methanol conversion using the syngas route (top) and direct partial oxidation (bottom). 
noble metal catalysts (pilot stage). ${ }^{12}$ The syngas is then converted to methanol using copper and zinc oxides on alumina as the catalyst. Although very efficient, and operating at high yields with high selectivity, the current technology is unsuitable for valorizing small scale methane sources. Given that an estimated $30-60 \%$ of sources are designated as 'stranded natural gas' under current economic conditions, ${ }^{13,14}$ this is an important issue. Technology to address the valorization of oil-associated gas, biogas and natural gas from remote wells is therefore highly desirable from an economic and an environmental point of view. Ideally, the oxidation of methane to methanol would be achieved in a single step at mild conditions, using oxygen as the ultimate electron acceptor (figure 3, bottom). With all products and reagents in the gas phase, the net conversion is exothermic, with $\Delta \mathrm{H}^{\circ}{ }_{\mathrm{r}}=-30.22 \mathrm{kcal} / \mathrm{mol}$ and $\Delta \mathrm{S}^{\circ}{ }_{\mathrm{r}}=-11.8 \mathrm{cal} / \mathrm{molK}$. Due to the negative reaction entropy, this process is favored at low temperatures (and feasible below $2500 \mathrm{~K}$ ). This is in contrast to the steam reforming of methane syngas (figure 3 , top), which is only thermodynamically possible above $1000 \mathrm{~K}$.

1.1.2 - Conversion of Benzene to Phenol. Phenol is an important precursor molecule for chemical synthesis due to its high reactivity toward electrophilic aromatic substitution. Downstream uses of phenol include production of bisphenol-A, ketonealcohol-oil (KA-oil) for nylon 66, and various polymers and pharmaceuticals. About $90 \%$ of phenol is currently produced from benzene using the cumene process. ${ }^{15}$ First, benzene undergoes a Friedel-Crafts alkylation with propylene (sometimes catalyzed using acidic zeolites). The resulting cumene is oxidized with molecular oxygen to form cumene hydroperoxide, which subsequently decomposes to a 1:1 mixture of acetone 
and phenol. ${ }^{16}$ Cumene hydroperoxide functions as a stable, partially oxidized intermediate, preventing over-oxidation. Drawbacks of the cumene process include the simultaneous production of acetone, which must be separated and for which a market must be found, as well as safety and environmental concerns regarding the intermediates (e.g., hydroperoxide) and solvents. As with the methane-to-methanol conversion, many of these issues can be addressed using a direct partial oxidation route, in which benzene is hydroxylated directly to form phenol.

The challenges associated with direct partial oxidation of benzene parallel those associated with the methane-tomethanol conversion. Like methane, benzene has an electronic structure that is chemically inert. The frontier molecular orbitals of benzene are shown in figure 4. The highest occupied molecular orbitals $\left(e_{g}\right)$ are $\pi$ bonding

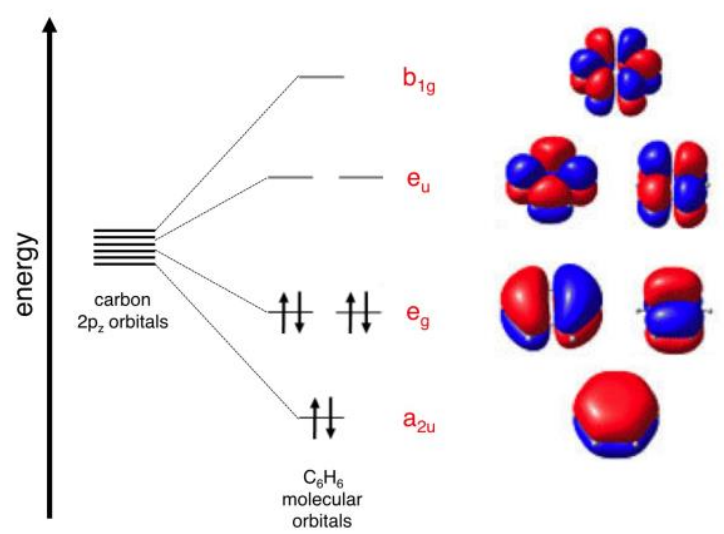

Figure 4. $\pi$ frontier molecular orbitals of benzene derived from out-of-plane carbon $2 p$ orbitals.

levels formed from linear combinations of the out-of-plane carbon $2 p$ orbitals. These orbitals are highly stable and delocalized due to aromatic $\pi$ bonding in the benzene ring. They are therefore resistant to electrophilic attack. The unoccupied antibonding orbitals ( $e_{u}$ in figure 4) are significantly destabilized and also delocalized, and this makes benzene resistant to nucleophilic attack. The $\mathrm{C}-\mathrm{H}$ bonds of benzene are even stronger than methane $\mathrm{C}-\mathrm{H}$ bonds $\left(113 \mathrm{kcal} / \mathrm{mol}\right.$ versus $104 \mathrm{kcal} / \mathrm{mol}$ for $\left.\mathrm{CH}_{4}\right)$, and as a result they are not susceptible to direct electrophilic attack through $\mathrm{H}$-atom abstraction. (On the other hand, the $\mathrm{C}-\mathrm{H}$ bonds of benzene are more susceptible to oxidative addition at 
low-valent metals than the $\mathrm{C}-\mathrm{H}$ bonds of methane due to the participation of aromatic $\pi$ orbitals in bonding to the metal. ${ }^{17}$ ) As with methane, metalloenzymes and metallozeolites overcome the chemical inertness of benzene using electrophilic reactive oxygen species. ${ }^{18,19}$ (Oxidative addition of aromatic $\mathrm{C}-\mathrm{H}$ bond is most facile at lowvalent $2^{\text {nd }}$ and $3^{\text {rd }}$ row transition metals, however these are scarce in biology and difficult to stabilize using protein-derived ligands). A key difference here is that these intermediates attack the $\pi$ bonding electrons of benzene ( $e_{g}$ in figure 4$)$ through an electrophilic aromatic substitution mechanism, and they do not interact directly with the $\mathrm{C}-\mathrm{H}$ bonds (in contrast to $\mathrm{H}$-atom abstraction from methane). A common mechanistic proposal for benzene hydroxylation by electrophilic metal-oxo species, not yet evaluated in the context of metallozeolite catalysis, involves initial formation of a so-called $\sigma$ complex. This intermediate is characterized by a single bond between the active oxygen and ipso carbon of the aromatic ring, which becomes $\mathrm{sp}^{3}$ hybridized. The formation of the new C-O bond is coupled to the one- or two-electron oxidation of the aromatic ring resulting in radical or cationic $\sigma$-complexes, respectively. Decay of the $\sigma$ complex to phenol may proceed through an inter- or intramolecular mechanism. Notable among these is the ' $\mathrm{NIH}$ ' shift, ${ }^{20}$ first defined for biological aromatic oxidations, in which the ipso proton of the $\sigma$-complex is transferred to the ortho carbon, forming 1,4-cyclohexadienone that subsequently tautomerizes to phenol.

1.2 - Enzymes as Industrial Oxidation Catalysts. In biology, enzymes exist that carry out selective hydrocarbon oxidation rapidly under ambient conditions. Methanotrophs have evolved iron and copper methane monooxygenases (particulate 
methane monooxygenase $-\mathrm{Cu}$, soluble methane monooxygenase $-\mathrm{Fe}$ ) to catalyze the hydroxylation of methane to methanol using atmospheric $\mathrm{O}_{2} \cdot{ }^{21}$ In all organisms, a range of iron and copper enzymes exist that perform aromatic hydroxylation with atmospheric $\mathrm{O}_{2}$, including cytochrome $\mathrm{p} 450$, phenylalanine hydroxylase, and tyrosinase. ${ }^{18,19}$ Comparison of current industrial oxidation catalysts to these enzymes demonstrates the considerable shortcomings of commercial synthetic systems. In terms of the catalytic activity per active site, measured by single site turnover frequencies, enzymatic systems are dramatically more efficient than catalysts used in today's industrial reactors. Typical turnover frequencies for enzymes range between $10^{3}$ and $10^{7} \mathrm{~s}^{-1}$ versus $10^{-2}$ to $10^{2} \mathrm{~s}^{-1}$ for synthetic catalysts. ${ }^{22}$ Moreover, enzymes showcase impressive product and reagent selectivity, often near $100 \%$, carefully tailored by evolution to exactly meet their host organism's needs. On the reagent side, this implies a reliance on readily available chemicals already present as, or synthesized from, ambient chemical compounds. On the product side, this implies largely innocuous side-products, if any. Bioreactors, using whole enzymes as catalysts, exploit these advantages, enabling certain processes, mainly relevant to the food industry, but also in the polymer industry, waste treatment industry, biomass conversion, and others, to run at mild conditions and attain high selectivity.

On the downside, enzymes suffer from a limited stability range with respect to numerous environmental parameters that are important in industrial oxidation chemistry. Having evolved to specifically support life at typically ambient conditions, enzymes lack the temperature, pressure and solvent tolerance required in most industrial processes. Especially environmental and energy related oxidation catalysis is often performed at 
elevated temperature and/or pressure and/or in the presence of highly concentrated contaminants not usually encountered in nature. This places prohibitive constraints on the use of enzymes in such applications. Additionally, precursors and products are generally not the same for industrial synthesis and biosynthesis, requesting some modifications of enzymatic processes at the catalyst level. Moreover, biocatalysts operate within the complex synergy provided by its host cell, often requiring specific cofactors such as ATP/ADP and $N A D(P)+/ N A D(P) H$. It is unfeasible to introduce such complexity in a reliable, large scale industrial reactor. Product separation from the multicomponent aqueous mixture in the bioreactor presents another complication. A review on alkane biohydroxylation covering the current status and prospects was published by Soussan et al. in $2016 .^{23}$

To overcome these challenges, while retaining the benefits of high activity and selectivity, a biomimetic approach has been followed in the past decades leading to interesting results with homogeneous complexes, ${ }^{24}$ synthetic enzymes, ${ }^{25}$ metal organic frameworks $s^{26,27}$ and encapsulated complexes in inorganic matrices, ${ }^{28-31}$ mimicking the ligand environment provided by the functional residues in the active pockets of enzymes. These systems have, along with the study of enzymes themselves, provided essential insight into the inner workings of enzymes, and in the methodology of the field. They still, however, lack the stability and simplicity required for supporting commercially viable, large-scale applications in industry.

1.3 - TMI Zeolites as Industrial Oxidation Catalysts. Zeolites offer many of the advantages associated with enzyme catalysis, including reactivity at well-defined 
transition metal active sites that can cycle through a range of oxidation states. Unlike enzymes, however, these inorganic materials are highly robust and can accommodate harsh reaction conditions. Zeolites have established a strong track record as industrial catalysts with initial applications as large scale acid catalysts for the petro-chemical industry in the 1960s. Incorporating extra framework transition metal ions into their inner pore structure (section 2) introduces these materials as highly stable oxidation catalysts, with properties reminiscent of those displayed by transition metal containing metalloenzymes (section 6). With this in mind, enzyme-inspired TMl-zeolite catalysts are promising materials to support the next generation of industrial oxidation processes. Here, we move from an enzyme mimicking approach towards one driven by concepts that have evolved from metalloenzyme chemistry.

The first TMI-zeolite active in the selective oxidation of methane to methanol was discovered by Panov et al. in $1990 .{ }^{32}$ They discovered the formation of an active Fe/O species on the ZSM-5 zeolite upon oxidation with $\mathrm{N}_{2} \mathrm{O}$. The reactive intermediate, named $\alpha-O$, has a remarkable activity towards the activation of methane. A bound intermediate is formed which can be extracted with water to yield methanol. Follow-up studies by the same group revealed the reactivity of $\alpha-O$ towards other hydrocarbons, including benzene. ${ }^{33}$ In contrast to methanol, no steam is required to release the phenol product, which desorbs spontaneously from the catalyst at $673 \mathrm{~K}$. The methane to methanol conversion over $\alpha-O$ continues to be burdened by practical limitations; the conversion is stoichiometric, requiring an additional steaming step for product desorption, and the requirement of $\mathrm{N}_{2} \mathrm{O}$ as oxidant limits the catalysts applicability. Meanwhile, the catalytic conversion of benzene to phenol has been translated into a 
pilot scale reactor by Solutia (now Ascend) in the AlphOx process, ${ }^{34}$ however the project was discontinued due to excessive deactivation of the catalyst by coking and a mismatch in recyclable $\mathrm{N}_{2} \mathrm{O}$ versus required amounts. Despite this, the practical potential of the catalyst is demonstrated by the AlphOx process, and new developments in research on the Fe-zeolites may well provide answers to the catalysts' current limitations including limited turnover numbers and low tolerance for trace impurities. Recently, for example, the geometric and electronic structure of $\alpha-O$ was defined by Snyder et al. ${ }^{35}$ (see section 5) - an essential step in enabling further application-directed engineering of the material.

Copper-containing zeolites show similar reactivity towards methane and benzene. ${ }^{36}$ While they are less reactive compared to the Fe-zeolites, Cu-zeolites have the distinct advantage of using $\mathrm{O}_{2}$ as oxidant. The reactive intermediate in selective hydrocarbon oxidation over $\mathrm{O}_{2}$-activated Cu-zeolites was first identified in 2005 by Groothaert et al. ${ }^{36}$, and its geometric and electronic structure was elucidated by Woertink et al. in $2009 .{ }^{37}$ As with the Fe-zeolites, issues related to product trapping and catalyst deactivation have presented barriers to commercialization. Emerging insight into $\mathrm{Cu}$ active sites in zeolites, discussed in section 4, will play an important role in resolving these issues.

For both iron and copper zeolites, parallels have been drawn to metalloenzyme chemistry with respect to function, but also with respect to active site geometric and electronic structure. Reliable experimental data on metallozeolite active sites that would enable this type of comparison are limited, however. Perhaps the most significant barrier with respect to experimental characterization of these materials is the presence 
of inactive spectator metals, which in some cases make up the majority of the metal content in TMI zeolites. As a result, data from bulk techniques such as X-ray absorption spectroscopy and magnetic susceptibility are not reliable. Despite this, bulk data have been interpreted extensively in the literature, resulting in a range of contradictory assignments for active sites in zeolites. In this review, we focus on recent advances enabled by site-selective spectroscopy, which limits interference from spectator metals. Insights into reactivity based on rigorous spectroscopy coupled to electronic structure calculations are presented, along with a critical comparison to iron and copper active sites in biology. 


\section{Copper and Iron Zeolites}

2.1 - Zeolite Structure and Properties. Zeolites are crystalline microporous silicates belonging to the group of the tectosilicates. The website of the International Zeolite Association (IZA) lists over 200 frameworks whose structures have been solved and recognized by IZA. The majority of these structures are synthesized, but some can be found in nature. The structures are labeled with a framework type code of 3 capital letters. The structures that are relevant to this review are shown in figure 5 together with their corresponding 3-letter codes. ${ }^{38}$ The ${ }^{*}$ BEA topology generally occurs as a disordered structure, consisting of an intergrowth of three polymorphs $\mathrm{A}, \mathrm{B}$ and $\mathrm{C}$, and is therefore given an asterisk prefix.

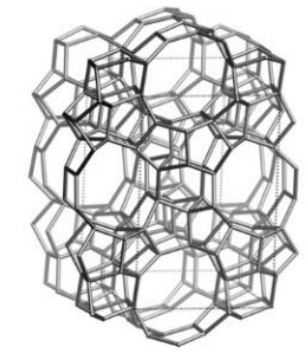

MFI

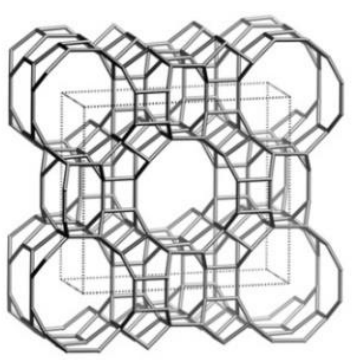

MOR

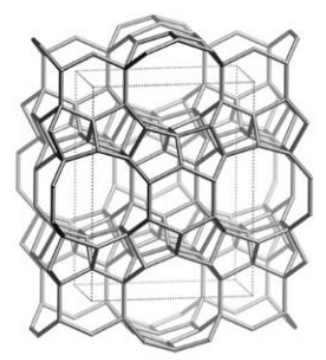

FER

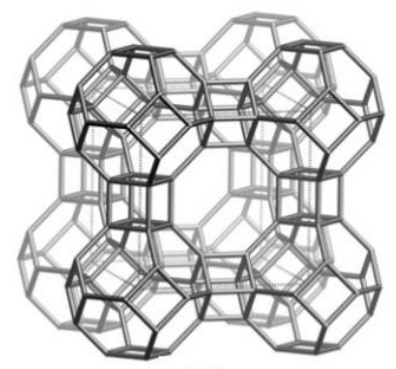

LTA

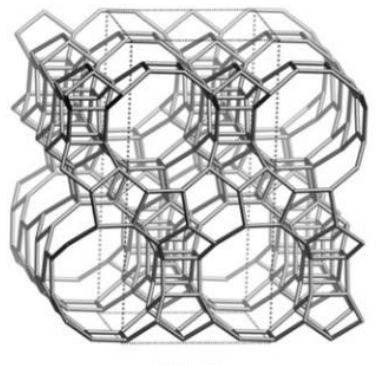

*BEA

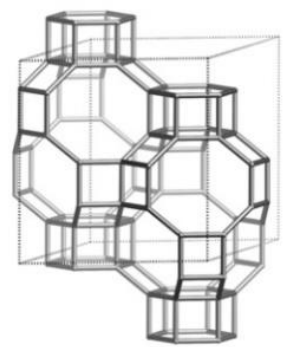

CHA

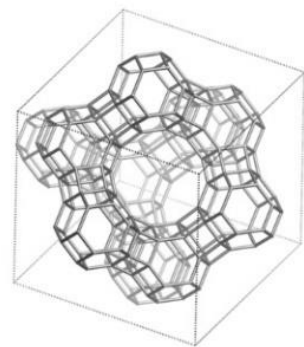

FAU

Figure 5. Framework structures of the MFI (includes ZSM-5), MOR, FER, LTA (includes zeolite A), *BEA, CHA (includes SSZ-13) an FAU (includes zeolites $X$ and $Y$ ) zeolite topologies. Adapted with permission from reference ${ }^{38}$. Copyright 2017 International Zeolite Association

A defining property of zeolites is the presence of channels and cages with free diameters of less than $2 \mathrm{~nm}$. These pores give the zeolites their characteristic microporosity. Channels may run in one, two or three directions, forming respectively 

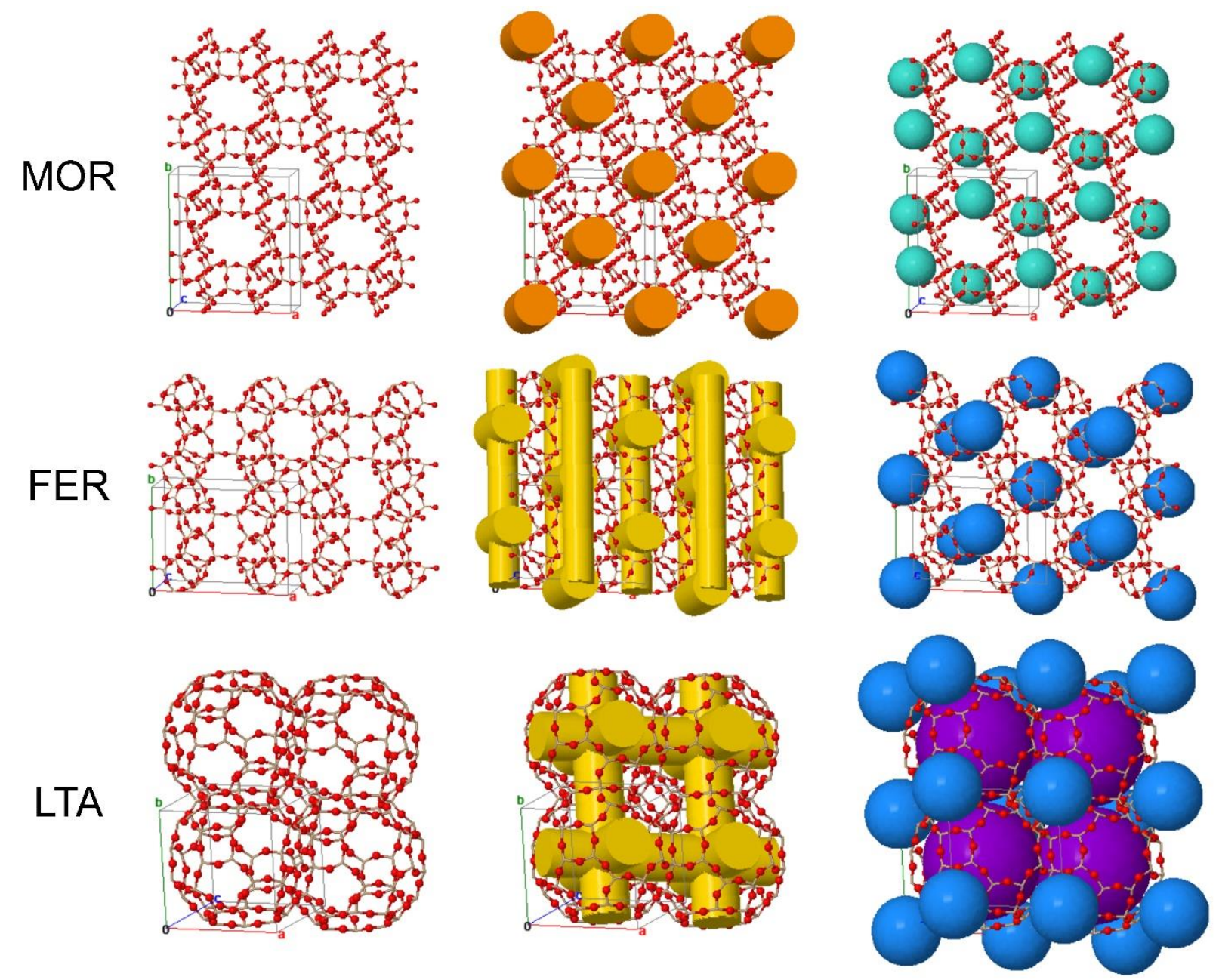

Figure 6. left: frameworks of the MOR, FER and LTA topologies; middle: channels forming the pore systems of the MOR (one-dimensional), FER (two-dimensional) and LTA (three-dimensional) topologies; right: and their cages, for MOR the side pockets are indicated (top right structure). ${ }^{39}$

one-, two, or three-dimensional pore systems, and the channels may or may not intersect. Access to the cages is dependent on passage through windows of $\mathrm{O}$ atoms, connecting the cages to the channels of the pore system. Channels and windows are characterized by the number of $\mathrm{O}$ atoms that form them, since this determines their free diameter. The pore systems of MOR (one-dimensional pore system), FER (twodimensional pore system) and LTA (tree-dimensional pore system) are shown in figure 6. ${ }^{39}$ The rings are symbolized with the notation $n \mathrm{MR}$ with $n$ the number of $\mathrm{O}$ atoms and MR abbreviating "membered ring". Figure 7 shows 6-, 8-, 10- and 12MRs and their 
corresponding free diameters. The latter differ somewhat depending on the Si:Al ratio of the zeolite lattice and on the structure type of the zeolite (vide infra). Thus a 6MR is
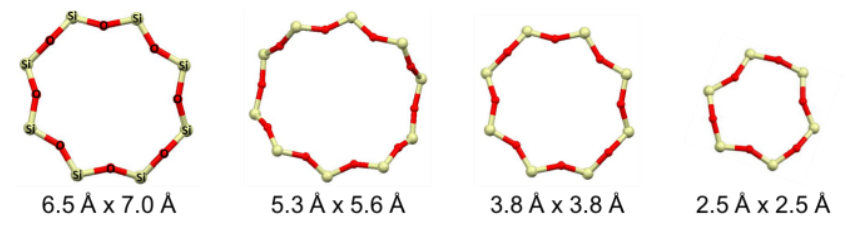

composed of six $(\mathrm{Si}, \mathrm{Al}) \mathrm{O}_{4}$ tetrahedra or

T-sites. For 8,10 and 12MRs, the number

Figure 7. typical rings and their free diameters of tetrahedra forming the ring or window (12MR, 10MR, 8MR, 6MR)

is 8,10 and 12 respectively. A molecule can only pass through a ring when the ring's free diameter equals or exceeds the dimensions of the molecule. This imposes steric constraints on the diffusion of molecules into the microporous network of the zeolite crystals towards the interior of the crystal, where the adsorption or catalytic sites are. Zeolites are therefore described as molecular sieves. An additional feature of zeolites is the isomorphous substitution of $\mathrm{Si}^{4+}$ by $\mathrm{Al}^{3+}$ in the lattice. This has two consequences; first, $\mathrm{Al}^{3+}$ is tetrahedrally coordinated to four lattice oxygen atoms and, because $\mathrm{Al}-\mathrm{O}$ bonds are longer than Si-O bonds (typically $0.187 \mathrm{~nm}$ vs. $0.163 \mathrm{~nm}$ ), this locally distorts the lattice and influences the shape and free diameter of the above mentioned rings. Second, as $\mathrm{Al}^{3+}$ carries one positive charge less than $\mathrm{Si}^{4+}$, the lattice becomes locally negatively charged. One formal negative charge is introduced per $\mathrm{Al}^{3+}$ substitution in the lattice. By Loewenstein's rule, $\mathrm{Al}^{3+}$ tetrahedra may never be in directly neigbouring positions in the framework, so that no Al-O-Al linkages are allowed. The upper limit of isomorphous substitution of $\mathrm{Si}^{4+}$ by $\mathrm{Al}^{3+}$ therefore yields a maximum $\mathrm{Si} / \mathrm{Al}$ ratio $=1$. Furthermore, the Takaishi and Kato rule dictates that only one $\mathrm{Al}^{3+}$ substitution is allowed in one 5MR and Dempsey's rule states that $\mathrm{Al}^{3+}$ substitution strives to an even distribution over the lattice. For synthetic zeoltites, however, kinetic parameters can cause deviations from the latter rule in zeolite crystallization. For selected zeolites, 
experimental studies have indicated that $\mathrm{Al}^{3+}$ substitutions are inclined to be in closer proximity than expected for a random distribution, but Loewenstein's rule remains valid. ${ }^{40}$ An illustration of the four possible aluminium configurations for the $6 \mathrm{MR}$ with $5>$ $\mathrm{Si} / \mathrm{Al}>1$ in the ${ }^{*} \mathrm{BEA}$ topology is given in figure 8 .

The negative lattice charge is compensated by exchangeable cations, giving rise to a cation exchange capacity (CEC) in agreement with the lattice Si/Al ratio. In principle, any cation can be exchanged provided its size is smaller than the free diameter of the channels and cages of the zeolite concerned. When protons are exchanged, Brönsted acid sites (BAS) are introduced as bridging [Al-OH-Si] hydroxyls between neighbouring $\mathrm{Si}^{4+}$ and $\mathrm{Al}^{3+}$.

The verification of Al distribution in the framework of silicon-rich zeolites is an experimental challenge, and is the subject of a 2012 review by Dedecek et al. ${ }^{41}$ They identify four types of Al substitution, based on the number of $\mathrm{Si}$ atoms $(n)$ separating

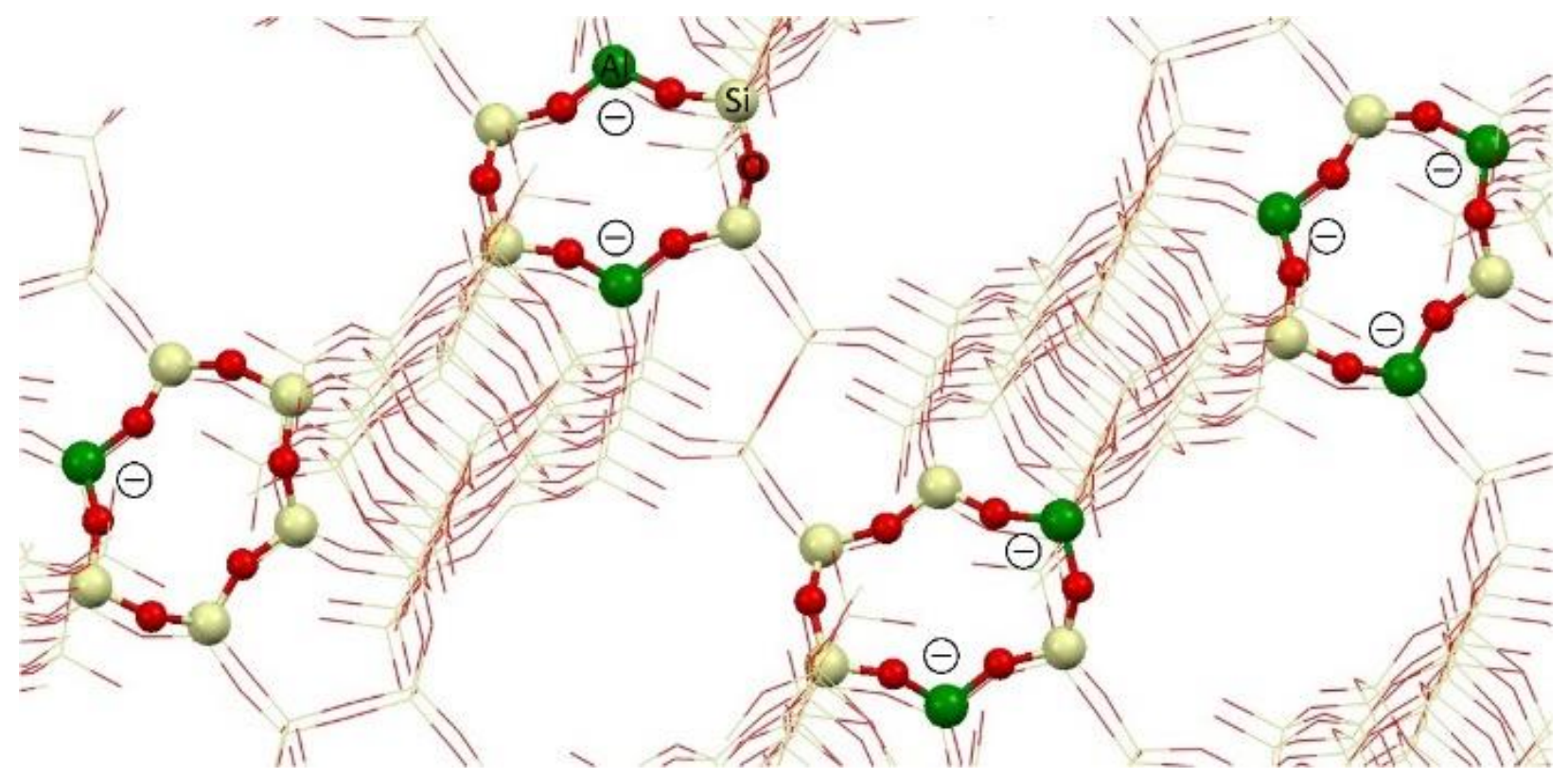

Figure 8. Examples of allowed $\mathrm{Al}$ distributions in $\beta-6 \mathrm{MR}$ sites, where qualitative rules on $\mathrm{Al}$ distributions in zeolites allow for one, two, or three Al T-sites. 
two nearest $\mathrm{Al}$ substitutions: (1) (Al-O-(Si-O) $\left.)_{n=1}-\mathrm{Al}\right)$ sequences, which are quantified with ${ }^{29} \mathrm{Si}-\mathrm{NMR}$; (2) $\left(\mathrm{Al}-\mathrm{O}-(\mathrm{Si}-\mathrm{O})_{2 \leq n \leq 3}-\mathrm{Al}\right)$ sequences in a single ring, which are quantified by NIR-UV-Vis spectroscopic quantification of $\mathrm{Co}^{2+}$ after subtraction of type (1); (3) (Al$\left.\mathrm{O}-(\mathrm{Si}-\mathrm{O})_{n \geq 2}-\mathrm{Al}\right)$ sequences not in a single ring, but within close distance, which are

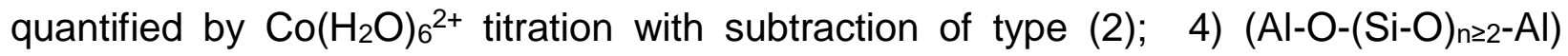
sequences not in a single ring and with the Al substitutions too separate to accommodate $\mathrm{Co}\left(\mathrm{H}_{2} \mathrm{O}\right)_{6}{ }^{2+}$, which make up the balance of total lattice Al. Note that the distinction between these four types is dependent on the assumption that $\mathrm{Co}^{2+}$ coordination only occurs at $\left(\mathrm{Al}-\mathrm{O}-(\mathrm{Si}-\mathrm{O})_{n \leq 3}-\mathrm{Al}\right)$ sequences in a single ring, which the authors based on a periodic DFT study on $\mathrm{Zn}^{2+}$ in MOR. Additionally, the distinction between types (3) and (4) is arbitrarily defined based on $\mathrm{Co}\left(\mathrm{H}_{2} \mathrm{O}\right)^{2+}$ exchange, and not directly on Al positioning.

Also for the exchange of TMI other than cobalt, (Al-O-(Si-O) $n \leq 3-\mathrm{Al})$ in a single ring, and (Al-O-(Si-O) $2-\mathrm{Al})$ sequences in 6MRs in particular, are of importance. These are particularly apt to accommodate divalent TMI. The occurrence of (Al-O-(Si-O)2-Al) sequences has been quantified for selected zeolite synthesis conditions by the method described in the previous paragraph, and is dependent on the Si:Al ratio, as illustrated in Table 1. 
Table 1. Number of Al-O-Si-O-Si-O-Al sequences for selected zeolites. ${ }^{40}$

zeolite Si:Al Unit cell composition ${ }^{a}$

ZSM5 12

ZSM5 30

FER 10

*BEA 15
$\mathrm{N}^{\circ}$ of Al-O-Si-O-Si-O-Al per unit cell

3.10

0.93

1.08

1.30

a:The variable cations are indicated by $\mathrm{M}$, where each $\mathrm{M}$ stands for a singly positively charged cation equivalent.

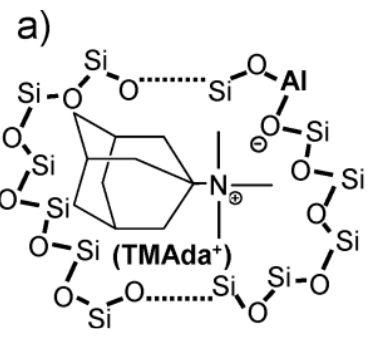

b)

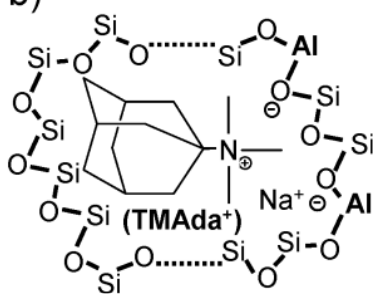

Di lorio et al. recently suggested a strategy for influencing the relative position of $\mathrm{Al}^{3+}$ substitution in CHA based on a steric / electrostatic effect. ${ }^{42}$

Figure 9. A) isolated $\mathrm{Al}^{3+}$ substitution in $\mathrm{CHA}$ synthesis in absence small cations $\left(\mathrm{Na}^{+}\right)$; $\left.\mathrm{B}\right)$ Nearby $\mathrm{Al}^{3+}$ substitution in $\mathrm{CHA}$ synthesis in presence of small cations $\left(\mathrm{Na}^{+}\right)$. Reproduced with permission from reference ${ }^{42}$. Copyright 2016 American Chemical Society.
Balancing the localized lattice charges in the growing crystallites with bulky N,N,Ntrimethyl-1-adamantylammonium

$\left(\mathrm{TMAda}^{+}\right)$cations would obstruct the approach of other TMDA+ cations and prohibit a second nearby lattice charge to be incorporated. Consequently, $\mathrm{Al}^{3+}$ substitutions are forced into a more separated configuration. Inclusion of smaller cations, such as $\mathrm{Na}^{+}$, on the other hand, allows the balancing of two charges in a single 8MR, thus allowing a nearly statistical distribution of $\mathrm{Al}^{3+}$ substitution (figure 9). The hypothesis was tested using the $\mathrm{Co}\left(\mathrm{H}_{2} \mathrm{O}\right)^{2+}$ titration method mentioned earlier, and is subject to the same assumptions. 
The crystallographic position of $\mathrm{Al}^{3+}$ substitution in the zeolite framework determines the siting of extra-framework cations in the pore system. While through refinement of X-rayand neutron diffractograms the positioning of T-sites is known for most zeolite topologies ${ }^{38}$, it remains a challenge to distinguish between $\mathrm{Al}$ and $\mathrm{Si} \mathrm{T}$-atoms with these methods. Zegenhagen et $\mathrm{al}^{43}$ succeeded in determining the $\mathrm{Al}^{3+}$ substitution in a scolecite zeolite using X-ray standing waves with a synchrotron source, but they did so on a highly perfect single crystal. For powder spectra, Rietveld refinement ${ }^{44,45}$, although seldom straightforward on zeolites due to intergrowth of different crystallographic phases and often large, complex unit cells, remains the best method to solve the structure. An indirect approach is to look at the crystallographic positioning of exchanged cations. Similarly, these can be located through XRD refinement methods such as Rietveld refinement and the maximum entropy method. ${ }^{46,47}$ Possible exchange sites, regardless of $\mathrm{Al}^{3+}$ position have been identified and tabulated for most common zeolites. $^{48,49}$ However, fractional occupancy of exchange sites, high diversity of exchange sites and the small number of extra-framework cations compared to atoms in the lattice prohibit a full account of the cations. ${ }^{48}$ Especially challenging are materials with high Si/Al and multivalent cationic species. High energy synchrotron sources alleviate these problems in part, but remain largely insufficient. Short range experimental techniques such as solid-state NMR, EXAFS, XANES, XPS, EPR, infrared spectroscopy, Raman, DR UV-Vis-NIR, and the use of probe molecules have assisted the interpretation of long range PXRD data. ${ }^{40,47,50,51}$ Lastly, theoretical work with Monte Carlo optimizations and periodic DFT models are used to further complement experiment. ${ }^{40,52}$ 
2.2 - TMI exchange. The most frequently applied method of loading TMI in zeolites is aqueous ion exchange of soluble TMI complexes. In dilute aqueous solution, TMI ions are fully hydrated (e.g. $\left.\left[\mathrm{M}\left(\mathrm{H}_{2} \mathrm{O}\right)_{6}\right]^{2+}\right)$ and can pass through the $8 \mathrm{MR}, 10 \mathrm{MR}$ and $12 \mathrm{MR}$ windows and channels but cannot pass through the 6MR windows. Aqueous ion exchange must be performed in strictly controlled $\mathrm{pH}$ conditions to avoid unwanted side reactions. At high $\mathrm{pH}$, silanol groups (point of zero charge $=6.0$ ) on the zeolite surface and defects are deprotonated and participate in the exchange. Besides this, TMI may precipitate at elevated $\mathrm{pH}$, and oligomeric $\mathrm{TMl}$ complexes formed in the solution are exchanged. These two phenomena lead to non-stoichiometric or over-exchanged zeolites. Stoichiometric exchange means that the positive charge of the TMI exchanged into the material exactly balances the negative charge of the lattice. For a divalent TMI, this implies that the molar ratio $\mathrm{TMI}: \mathrm{Al}=0.5$. Upon over-exchange, this ratio exceeds 0.5 , and the excess charge is balanced by other negative charges, not related to framework aluminium. At exceedingly low $\mathrm{pH}$, exchange is hampered by competitive exchange of $\mathrm{H}^{+}$and TMI. As a result, mixed $\left(\mathrm{H}^{+}, \mathrm{TMI}\right)$-zeolite is obtained.

$\mathrm{Cu}^{2+}$ precipitates at $\mathrm{pH}>7.0$. The recommended $\mathrm{pH}$ for aqueous $\mathrm{Cu}^{2+}$ ion exchange is $4.0<\mathrm{pH}<6.0$, which is a feasible range for material preparation. For $\mathrm{Fe}^{3+}$, however, aqueous exchange of $\left[\mathrm{Fe}\left(\mathrm{H}_{2} \mathrm{O}\right)_{6}\right]^{3+}$ is inevitably accompanied with unwanted side reactions because $\mathrm{Fe}^{3+}$ precipitates at $\mathrm{pH} \geq 2.2$.

To avoid side reactions, three other strategies have been explored. (1) Exchange of $\mathrm{Cu}^{+}$ and $\mathrm{Fe}^{2+}$. This is a cumbersome procedure because these exchanges have to be 
performed in the strict absence of $\mathrm{O}_{2}$. (2) Solid state ion exchange or heating a mechanical mixture of - preferably - a H-zeolite and a salt of the TMI with a relatively low melting point. The exchange reactions can be written as:

$\mathrm{CuCl}_{2}+\mathrm{H}$-zeolite $\rightarrow$ Cu-zeolite $+2 \mathrm{HCl}_{(\mathrm{g})}$

$\mathrm{FeCl}_{3}+\mathrm{H}$-zeolite $\rightarrow$ Fe-zeolite $+3 \mathrm{HCl}_{(\mathrm{g})}$

Water must be avoided, as it leads to an acidic solution in the channels and cavities that may attack the lattice resulting in local lattice breakdown. (3) the third technique is impregnation of a TMI complex dissolved in an organic solvent into the zeolite. The ligands of the complex have to be removed by calcination. Impregnation of $\mathrm{Fe}(\mathrm{acac})_{3}$ $($ acac $=$ acetylacetonate $)$ in toluene, for example, was employed to prepare the $\alpha-F e$ sites discussed in section 5.2.1. Upon calcination, the acac ligands are decomposed to $\mathrm{CO}_{2}$ and $\mathrm{H}_{2} \mathrm{O}$, and $3 \mathrm{H}^{+}$of the zeolite are consumed in this process:

$\mathrm{Fe}(\mathrm{acac})_{3} / \mathrm{H}$-zeolite $+17.5 \mathrm{O}_{2} \rightarrow \mathrm{Fe}^{3+}$-zeolite $+15 \mathrm{CO}_{2}+11 \mathrm{H}_{2} \mathrm{O}$.

At high Fe loadings, where the zeolite charge is insufficient to host the impregnated TMI, $\mathrm{Fe}_{2} \mathrm{O}_{3}$ and $\mathrm{Fe}_{3} \mathrm{O}_{4}$ nanoparticles are formed.

Regardless of the strategy to load TMI into the zeolite, the TMI complexes have to diffuse through channels and cavities to ensure a homogeneous distribution of the TMI in the zeolitic crystals. 
2.3 - Activation and Autoreduction. Activation is a high temperature treatment aimed at removing all volatile inorganic and organic molecules adsorbed in the microporous system of zeolites. As ion exchange is usually performed in water, activation results in most cases in the removal of adsorbed water as well.

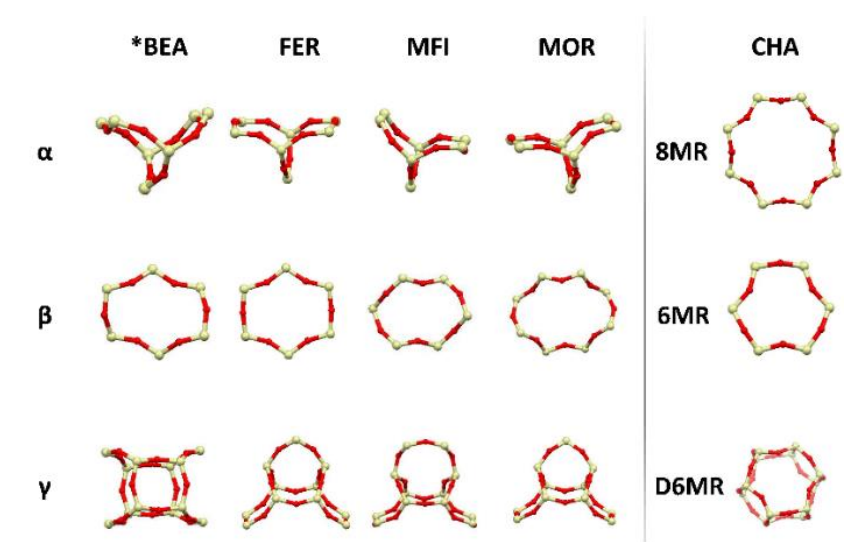

Figure 10. Common exchange sites for TMI after calcination for the ${ }^{*} \mathrm{BEA}, \mathrm{FER}, \mathrm{MFI}, \mathrm{MOR}$ and $\mathrm{CHA}$ topologies. Reproduced from reference ${ }^{53}$ with permission. Copyright 2000 Elsevier.

Organic molecules originating from ion exchange with organic salts and organic templates from zeolite synthesis are desorbed and/or decomposed. In the latter case the decomposition products are desorbed. When the ligands are removed by calcination/activation, the naked TMI coordinate to lattice oxygen atoms in the vicinity of the negative lattice charges - i.e. the Al tetrahedral (vide supra). Common TMI exchange sites for relevant topologies are shown in figure $10^{40,53}$, and these are subject to aluminium substitution as described in section 2.1.

After the removal of organic ligands and water, further temperature elevation in vacuo or in inert atmosphere results in bare TMI coordinated to lattice oxygens. Two side reactions can occur 
First, in over-exchanged zeolites, condensation leads to polynuclear TMI species of the type $\left[\mathrm{Mx}^{\mathrm{n}+} \mathrm{O}_{\mathrm{y}}\right]^{\mathrm{xn}-2 \mathrm{y}}(\mathrm{x}=1,2,3)$, and a zeolitic material containing a range of $\mathrm{TMl}$ clusters from monoatomic to oxidic crystals at the external surface of the zeolite crystals. Thermodynamic calculations indicate that larger copper oxide clusters, at least up to pentamers, are more stable compared to smaller clusters when embedded in Cu-MOR and Cu-MFI due to the extra stabilization by an increased degree of oxygen coordination. ${ }^{54}$ Consequently, copper clusters with increasing nuclearity become increasingly abundant at higher copper loadings. At over-exchange $\left(\mathrm{Cu}^{2+} / \mathrm{Al}>0,5\right)$ on $\mathrm{H}-\mathrm{MOR}, \mathrm{CuO}_{x}$ nanocrystals were detected with transmission electron microscopy (TEM) and single Al sites were compensated by monovalent $\left[\mathrm{Cu}^{2+} \mathrm{OH}^{-}\right]^{+}$species. ${ }^{55}$ Cluster growth in the MOR zeolite was found to be most favourable in the side-pockets (figure 6) with two symmetrically oriented aluminium tetrahedral on opposite sides of the 8-MR channel. ${ }^{54}$ In applications, it is commonly desirable to specifically stabilize a single TMI species. Other TMI species, not relevant to the intended purpose, are seen as 'spectator' species and are best avoided by careful control of the exchange parameters. Often, high active site purity is achieved by limiting the loading of the TMI on the zeolite to very dilute concentrations. Developing strategies to extrapolate high active site purity to elevated metal loadings will be key in improving space-time yield and catalyst productivity for TMI zeolites to industrially relevant levels.

In the second side reaction, $\mathrm{Fe}^{3+}$ and $\mathrm{Cu}^{2+}$ zeolites undergo an autoreduction upon activation in inert atmosphere; this is the reduction of $\mathrm{Cu}^{2+}$ to $\mathrm{Cu}^{+}$and $\mathrm{Fe}^{3+}$ to $\mathrm{Fe}^{2+}$ 
accompanied by oxidation of $\mathrm{O}^{2-}$ ions to $\mathrm{O}_{2}$. The $\mathrm{O}^{2-}$ ions may originate from the lattice,

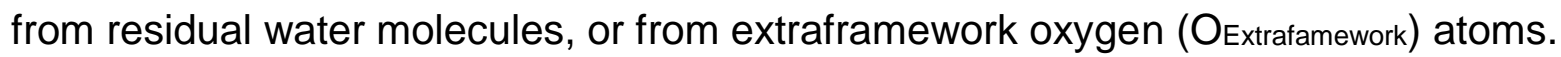

For the autoreduction of $\mathrm{Fe}^{3+}$, one may formally write:

$2[\mathrm{Fe}-\mathrm{OH}]^{2+} \rightarrow[\mathrm{Fe}-\mathrm{O}-\mathrm{Fe}]^{2+}+\mathrm{H}_{2} \mathrm{O}(\mathrm{g})$

$[\mathrm{Fe}-\mathrm{O}-\mathrm{Fe}]^{2+} \rightarrow 2 \mathrm{Fe}^{2+}+0.5 \mathrm{O}_{2}(\mathrm{~g})$

Autoreduction of $\mathrm{Fe}^{3+}$ to $\mathrm{Fe}^{2+}$ in zeolites was determined to range between 63 and $67 \%$ of the total Fe content ${ }^{56,57}$, although Sobalik et al. ${ }^{58}$ obtained $81-96 \%$. This difference can probably be ascribed to differences in introduction of Fe in the zeolite. These values are, however, obtained from zeolites with low Fe loadings of $7-14 \%$ of the cation exchange capacity (CEC) and even then several $\mathrm{Fe}^{2+}$ species were identified. Higher levels of Fe loading lead to the formation of oligomeric Fe species, complicating the chemistry of autoreduction strongly since both isolated $\mathrm{Fe}^{3+}$ ions and $\mathrm{Fe}_{2} \mathrm{O}_{3}$ and $\mathrm{Fe}_{3} \mathrm{O}_{4}$ are subject to autoreduction.

$6 \mathrm{Fe}_{2} \mathrm{O}_{3} \rightarrow 4 \mathrm{Fe}_{3} \mathrm{O}_{4}+\mathrm{O}_{2}(\mathrm{~g})$

$2 \mathrm{Fe}_{3} \mathrm{O}_{4} \rightarrow 6 \mathrm{FeO}+\mathrm{O}_{2}(\mathrm{~g})$

To avoid these complications, Snyder et al. ${ }^{35}$ obtained $\mathrm{Fe}^{2+}$ zeolites with close to $100 \%$ of $\mathrm{Fe}^{2+}$ by autoreduction in $\mathrm{He}$ at $900^{\circ} \mathrm{C}$, followed by $\mathrm{H}_{2}$ reduction at $700^{\circ} \mathrm{C}$. The total amount of $\mathrm{Fe}^{2+}$ was determined by Mössbauer to represent more than $90 \%$ of the total 
Fe content. Three types of $\mathrm{Fe}^{2+}$ species were found: $\mathrm{Fe}^{2+}(1)$ with a $\mathrm{d}-\mathrm{d}$ band envelope around $5000 \mathrm{~cm}^{-1} ; \mathrm{Fe}^{2+}(2)$ with a d-d band envelope in the region around $9000 \mathrm{~cm}^{-1}$ and $\mathrm{Fe}^{2+}(3)$ with a d-d band at $15900 \mathrm{~cm}^{-1}$. The former two species haven't been studied in detail, but $\mathrm{Fe}^{2+}(3)$ has been characterized by magnetic circular dichroism (MCD) and Mossbauer spectroscopy and assigned to a square planar-coordinated $\mathrm{Fe}^{2+}$. (see section 5.2.2).

For the autoreduction of $\mathrm{Cu}^{2+}$, the following reactions have been proposed in literature 59-61:

$2[\mathrm{Cu}-\mathrm{OH}]^{+} \rightarrow[\mathrm{Cu}-\mathrm{O}-\mathrm{Cu}]^{2+}+\mathrm{H}_{2} \mathrm{O}(\mathrm{g})$

$[\mathrm{Cu}-\mathrm{O}-\mathrm{Cu}]^{2+} \rightarrow 2 \mathrm{Cu}^{+}+0.5 \mathrm{O}_{2}(\mathrm{~g})$

Alternatively, one may write

$2[\mathrm{Cu}-\mathrm{OH}]^{+} \rightarrow \mathrm{Cu}^{+}+\mathrm{Cu}^{2+}-\mathrm{O}^{-}+\mathrm{H}_{2} \mathrm{O}(\mathrm{g})$

$\mathrm{Cu}^{+}+\mathrm{Cu}^{2+-} \mathrm{O}^{-} \rightarrow 2 \mathrm{Cu}^{+}+0.5 \mathrm{O}_{2}(\mathrm{~g})$

The released oxygen molecules are derived from the $[\mathrm{Cu}-\mathrm{OH}]^{+}$species above $523 \mathrm{~K}$ in vacuo or inert atmosphere. In early research on $\mathrm{Cu}-\mathrm{Y}$ zeolites (FAU topology figure 5, $\mathrm{Si} / \mathrm{Al} \geq 1.5)$ Beyer and Jacobs ${ }^{62}$ proposed that the reduction of $\mathrm{Cu}^{2+}$ to $\mathrm{Cu}^{+}$is 
accompanied by release of lattice oxygens and Al from the lattice. The lattice oxygens desorb as $\mathrm{O}_{2}$ and $\mathrm{Al}$ takes an extraframework position.

The autoreduction has been quantified for Cu-MOR and Cu-MFI. Cu-MOR, activated in $\mathrm{O}_{2}$, contains three distinct $\mathrm{Cu}^{2+}$ species ${ }^{63}$ : two are isolated (non-interacting) $\mathrm{Cu}^{2+}$ ions with respectively $g_{\|}=2.32$ and $g_{\|}=2.27$. The third is a bent dinuclear oxo complex: $[\mathrm{Cu}-\mathrm{O}-\mathrm{Cu}]^{2+}$. Upon heating in vacuo $(\mathrm{He})$ at $180^{\circ} \mathrm{C}(453 \mathrm{~K}), \mathrm{O}_{2}$ is released and both $\mathrm{Cu}^{2+}$ with $\mathrm{g}_{\|}=2.27$ and $[\mathrm{Cu}-\mathrm{O}-\mathrm{Cu}]^{2+}$ are reduced to $\mathrm{Cu}^{+}$in agreement with the reactions given above. On a sample with $\mathrm{Cu} / \mathrm{Al}=0.43,0.50 \mathrm{mmol} / \mathrm{g}$ of $\mathrm{Cu}^{2+}$ are reduced in total: $0.07 \mathrm{mmol} / \mathrm{g}$ isolated $\mathrm{Cu}^{2+}$ and $0.43 \mathrm{mmol}$ of $\mathrm{Cu}^{2+}$ in the form of the dinuclear complex. This amounts to $70 \%$ of the total Cu content.

Moretti et al. ${ }^{64}$ quantified the amount of $\mathrm{Cu}^{+}$formed in the autoreduction reaction of $\mathrm{Cu}$ ZSM-5 (MFI topology, figure 6) by measuring the amount of $\mathrm{Cu}-\mathrm{CO}$ complexes and the amount of irreversibly adsorbed $\mathrm{N}_{2}$. The CO measurement resulted in $80 \%$ of the total amount of $\mathrm{Cu}$ as monovalent $\mathrm{Cu}^{+}$, irrespective of the exchange level or the $\mathrm{Cu}$ content.

2.4 - Factors Influencing Active Site Nuclearity. The formation of a bent dinuclear $[\mathrm{Cu}-\mu-\mathrm{O}-\mathrm{Cu}]^{2+}$ complex upon $\mathrm{O}_{2}$ activation contrasts with the formation of $\left[\mathrm{Cu}_{3}(\mu-\mathrm{O})_{3}\right]^{2+}$ in absence of spectators (i.e. single-site tricopper) reported by Grundner et al. ${ }^{55}$ upon $\mathrm{O}_{2}$ activation of $\mathrm{Cu}-\mathrm{MOR}$ and $\mathrm{Cu}-\mathrm{MFI}$. Both were ion-exchanged with $0.01 \mathrm{M}$ aqueous copper(II)acetate. Two differences in the preparation of both materials can, however, be identified. First, the $\left[\mathrm{Cu}_{3}(\mu-\mathrm{O})_{3}\right]^{2+}$ materials were exchanged from a parent material in the proton form (H-MOR and $\mathrm{H}-\mathrm{MFI})$, as opposed to a parent material in the sodium form (Na-MOR and Na-MFI) for the $[\mathrm{Cu}-\mu-\mathrm{O}-\mathrm{Cu}]^{2+}$ materials. ${ }^{36}$ Second, the exchange 
was buffered to a $\mathrm{pH}=5.7$ to obtain the $\left[\mathrm{Cu}_{3}(\mu-\mathrm{O})_{3}\right]^{2+}$ material. For the $[\mathrm{Cu}-\mu-\mathrm{O}-\mathrm{Cu}]^{2+}$ materials, the exchange was not buffered. ${ }^{36}$ The $\left[\mathrm{Cu}_{3}(\mu-\mathrm{O})_{3}\right]^{2+}$ materials were reported to stabilize only single-site tricopper clusters in $\mathrm{Cu}, \mathrm{H}-\mathrm{MOR}$ from 0 up to $400 \mu \mathrm{mol}$ copper per gram of catalyst $(\mathrm{Si} / \mathrm{Al} \approx 12) \cdot{ }^{55}$ Compared to previous literature, this is a high concentration of single copper active sites to obtain on a zeolite. The dinuclear $\left[\mathrm{Cu}_{2} \mathrm{O}\right]^{2+}$ sites in ZSM-5 are present in concentrations levelling off around $720 \mu \mathrm{mol}$ copper per gram ${ }^{65}$, but with less than $10 \%$ of this copper forming the active sites. ${ }^{66}$

Titration of Brönsted acid sites on H-MOR with pyridine and hexane before and after copper exchange indicates a selective stabilization of the $\left[\mathrm{Cu}_{3}(\mu-\mathrm{O})_{3}\right]^{2+}$ clusters in an $8 \mathrm{MR}$ in the MOR side pocket (figure 6). The high concentration of Al substitution in the $8 \mathrm{MR}$ of MOR is expected to play a key role in the selective stabilization of the $\left[\mathrm{Cu}_{3}(\mu-\right.$ O) $\left.{ }_{3}\right]^{2+}$ clusters and the specific lattice environment is hypothesized to act as a directing agent towards the trinuclear clusters. ${ }^{67}$ This is in agreement with observations on $\mathrm{Cu}, \mathrm{H}$ ZSM-5, where Al pairs are mainly located in the $6 \mathrm{MR}$ and other rings tend to hold only a single substitution with Al. ${ }^{40,68,69}$ In this case, the self-organization into trinuclear sites is only initiated at a $0.20 \mathrm{Cu} / \mathrm{Al}$ atomic ratio. Similarly, a $\mathrm{Cu} / \mathrm{Al} \geq 0.20$ ratio on $\mathrm{Na}-\mathrm{ZSM}-5$ is required to form the $[\mathrm{Cu}-\mu-\mathrm{O}-\mathrm{Cu}]^{2+} \cdot{ }^{36}$ Below this threshold, isolated $\mathrm{Cu}^{2+}$ are energetically preferred in the doubly $\mathrm{Al}^{3+}$ substituted $6 \mathrm{MR}$ of MFI. ${ }^{67}$ The importance of aluminium pairing was further demonstrated by comparing copper speciation on $\mathrm{H}$ ZSM-5 samples with a different population of exchange sites containing Al-O-(Si-O)n ${ }_{n \leq 3-}$ Al sequences. At optimal copper loading, the concentration of $\left[\mathrm{Cu}_{3}(\mu-\mathrm{O})_{3}\right]^{2+}$ was lower on the sample with less such sequences and the speciation was more heterogeneous. ${ }^{67}$ 
Similarly, in Cu-SSZ-13 (CHA topology, Si/Al > 5), bare divalent $\mathrm{Cu}^{2+}$ cations first occupy the most stable positions at doubly substituted 6MR sites. At higher loadings, $[\mathrm{Cu}(\mathrm{OH})]^{+}$complexes, associated with low temperature SCR activity ${ }^{70-72}$ and methane activation ${ }^{73,74}$, are formed in the $8 \mathrm{MR}$ exchange sites of $\mathrm{CHA}$ with a single aluminium substitution..$^{70-72,75,76}$

In ZSM-5, a decrease in the formation of trinuclear clusters was observed in the presence of alkali- $\left(\mathrm{Na}^{+}, \mathrm{K}^{+}\right)$and alkali-earth metal $\left(\mathrm{Mg}^{2+}, \mathrm{Ca}^{2+}\right)$ cations. ${ }^{77,78}$ Instead, a more heterogeneous mixture of copper species was obtained ${ }^{67}$. The formation of dinuclear copper clusters is observed in spite of ab initio thermodynamic calculations which indicate a higher stability of trinuclear species in Cu-MOR ${ }^{55}$ and Cu-ZSM-5 ${ }^{79}$. It is known that, like $\mathrm{Cu}^{2+}, \mathrm{Na}^{+}$preferentially coordinates in the MOR side-pockets ${ }^{80}$, while Brönsted acid sites are better accommodated in the unconfined 12MR channels of MOR. ${ }^{81-83}$ Possibly, the presence of $\mathrm{Na}^{+}$avoids further self-assembly of dinuclear copper into trinuclear species.

$A b$ initio thermodynamic analyses reveal that TMI behaviour under operating conditions may differ significantly from standard characterization conditions. Atmosphere composition and temperatures are expected to be of influence. ${ }^{76}$ Furthermore, the presence of water hydrating the copper cations may mobilize the extra-framework cations enabling a redistribution over the lattice. ${ }^{61,71,75,77}$ The $[\mathrm{Cu}(\mathrm{OH})]^{+}$species in $\mathrm{Cu}$ SSZ-13 is suggested to only be stable at high temperatures $\left(>250^{\circ} \mathrm{C}\right)$ in oxidizing conditions. In inert atmosphere tri-coordinated $[\mathrm{Cu}(\mathrm{OH})]^{+}$autoreduces to form bare, two- 
coordinated $\mathrm{Cu}^{+}$. The autoreduction can be reversed in an $\mathrm{O}_{2}$ atmosphere with $5 \% \mathrm{H}_{2} \mathrm{O}$ at $400^{\circ} \mathrm{C} .{ }^{72}$ It is interesting to note here that $[\mathrm{Cu}(\mathrm{OH})]^{+}$is predicted by DFT to be present in a limited amount $(\sim 10 \%)$ within a temperature range up to $450^{\circ} \mathrm{C}$ in $\mathrm{O}_{2}$ atmosphere with 0.5 to $5 \% \mathrm{H}_{2} \mathrm{O}$. This is in agreement with the fraction of active copper estimated as water-resistant active sites in methane to methanol conversion estimated on Cu-SSZ-13 by Wulfers et al. ${ }^{84}$ 

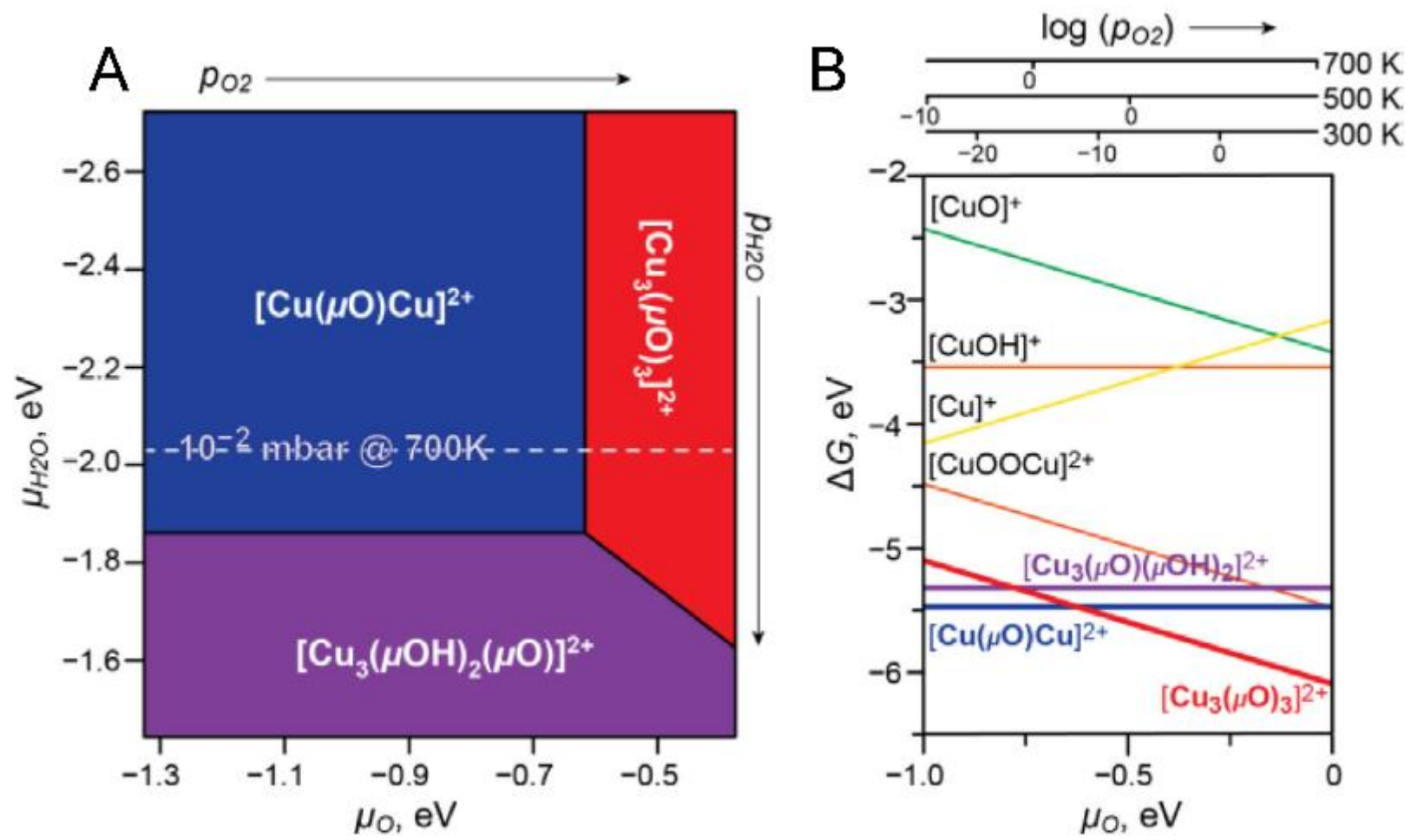

Figure 11. A) Most thermodynamically stable Cu-oxo clusters in Cu-ZSM-5 zeolite under different conditions of temperature, oxygen partial pressure and steam partial pressure as determined by ab initio thermodynamic analysis. B) Cross-section of the 3D phase diagram with $\mu_{\mathrm{H} 2 \mathrm{O}}$ fixed at $10^{-2} \mathrm{mbar}$ at $700 \mathrm{~K}$ (dashed line in A). Reproduced from reference ${ }^{79}$ with permission. Copyright 2016 Elsevier.

On a comparable note, trinuclear copper cluster stability on $\mathrm{Cu}, \mathrm{H}-\mathrm{ZSM}-5$ is calculated to exceed the stability of binuclear complexes in $\mathrm{O}_{2}$ atmosphere. The order of stability is, however, reversed in an $\mathrm{O}_{2}$ deprived atmosphere (figure 11). ${ }^{79}$ This result can be expected to have an impact on the correct characterization of these sites when they are implemented in reducing reaction conditions.

For iron zeolites, the factors which govern the formation of multinuclear sites remain largely unknown, but it is known that heat treatment, atmosphere composition and iron loading are influential for iron dispersion. ${ }^{85-87}$ Moreover, the exact nuclearity of active oligomers has, to our knowledge, never been directly detected with site selective spectroscopy. Dimeric iron sites have long been proposed as the active sites for $\mathrm{N}_{2} \mathrm{O}$ decomposition and $\mathrm{CH}_{4}$ activation. ${ }^{57,58,88,89}$ This proposal was inspired by the dimeric 
active site in the sMMO enzyme, and further support was given by the average nuclearity derived from EXAFS results as well as TPR experiments. ${ }^{90,91}$ At elevated iron loading, speciation between different oligomers becomes prohibitively complex for characterization and any connection between specific iron sites and reactivity becomes difficult to determine. In Fe-ZSM-5, Hammond et al. ${ }^{92,93}$ have suggested the presence of a binuclear di- $\mu$-hydroxo bridged Fe complex $\left(\left[\mathrm{Fe}_{2}\left(\mu_{2}-\mathrm{OH}\right)_{2}\left(\mathrm{H}_{2} \mathrm{O}\right)_{2}\right]^{2+}\right)$ with an Fe-O-Fe symmetric stretch at $521 \mathrm{~cm}^{-1}$ in the Raman spectrum, though larger nuclearity sites could not be excluded. The $521 \mathrm{~cm}^{-1}$ feature was observed to dissipate after reaction with aqueous $\mathrm{H}_{2} \mathrm{O}_{2}$, after which the material is active in the low temperature oxidation of methane. Can Li et al. ${ }^{94}$ linked a Raman feature at $867 \mathrm{~cm}^{-1}$ to a v(O-O) stretch of a peroxo bridged binuclear iron site active in benzene hydroxylation on Fe-ZM-5. Similarly, a $730 \mathrm{~cm}^{-1}$ was assigned to a peroxo stretch associated with a binuclear iron complex in ZSM-5 by Sachtler et al. ${ }^{95}$ The large variability in sample preparation and pretreatment makes comparison difficult, and more study on the aspects of iron introduction and pretreatment is needed for better understanding. Reliable spectroscopic probes of various iron sites would be instrumental to derive the workings of iron speciation in zeolites.

2.5 - Coordination of Divalent Metal Ions in Dehydrated Zeolites The study of the coordination of TMI in zeolites was initiated by Klier and coworkers. ${ }^{96,97}$ They developed a ligand field model to explain the $d-d$ spectra of the $3 d$ series of TMI based on the ligand field transitions of $\mathrm{Co}^{2+}$ and $\mathrm{Ni}^{2+}$ in dehydrated zeolite A (LTA topology figure 5). The TMI were located on the trigonal axis of a symmetrical $6 \mathrm{MR}$ with three $\mathrm{O}$ atoms of 
the $6 \mathrm{MR}$ in their first coordination sphere. The point symmetry is $\mathrm{D}_{3 \mathrm{~h}}$ if the $\mathrm{TMI}$ are located in the plane of the three $\mathrm{O}$ atoms; while it is $\mathrm{C}_{3 v}$ for the TMI located slightly above the plane of the three $\mathrm{O}$ atoms. This model was extended by the group of Schoonheydt using a spin Hamiltonian analysis to include the EPR parameters of $\mathrm{Cu}^{2+}$ in FAU, LTA and MOR. ${ }^{98}$ In $\mathrm{C}_{3 \mathrm{v}}, \mathrm{Cu}^{2+}$ acquires a doubly degenerate ground state and is subject to the Jahn-Teller (JT) effect. This was visualized by allowing $\mathrm{Cu}^{2+}$ ions to move off the trigonal axis.

These Ligand Field (LF) models had two major disadvantages: First, no distinction was made between Al- and Si-tetrahedra forming the 6MR; second, the zeolite lattice was strictly rigid; only the TMI were allowed to move so as to find a match between the theoretical $d-d$ bands and the experimental spectra. The groups of Schoonheydt and Pierloot ${ }^{99-102}$ developed a more detailed coordination model for TMI in zeolites, in particular for $\mathrm{Cu}^{2+}$ and $\mathrm{Co}^{2+}$. These efforts were later extended to $\mathrm{Fe}^{2+}$ coordination in the $6 \mathrm{MRs}$ of zeolite beta (see section 5.2 for detail). In this section, the focus will be on coordination of $\mathrm{Cu}^{2+}$ to $6 \mathrm{MRs}$ in dehydrated zeolites, where models have been optimized within the frame of DFT (with all the Si and Al tetrahedra included). D-d transitions and the EPR g-parameters were calculated on these optimized structures using complete active space perturbation theory (CASPT2). Typical DFT optimized coordination geometries are shown in figure 12, with the corresponding CASPT2predicted g-values and ligand field transitions given in table 2. 

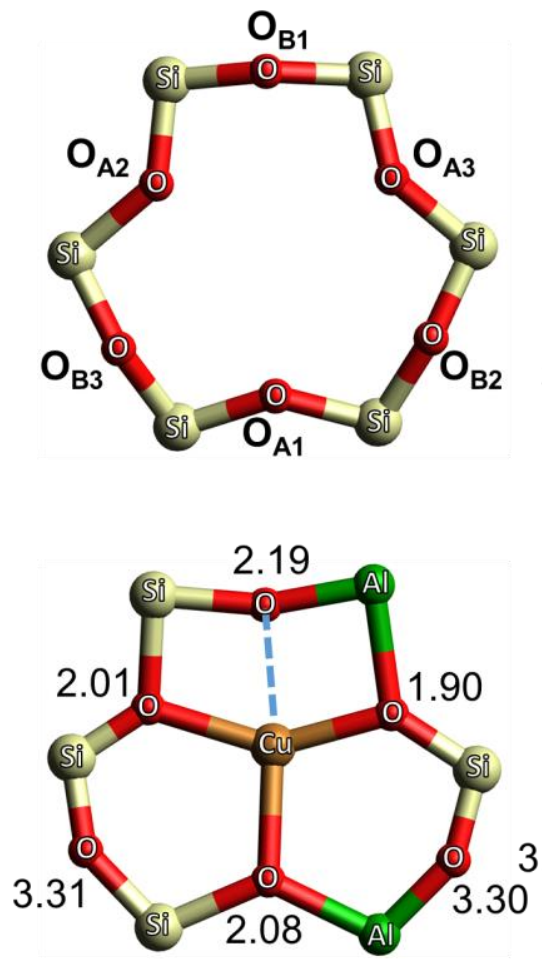

C

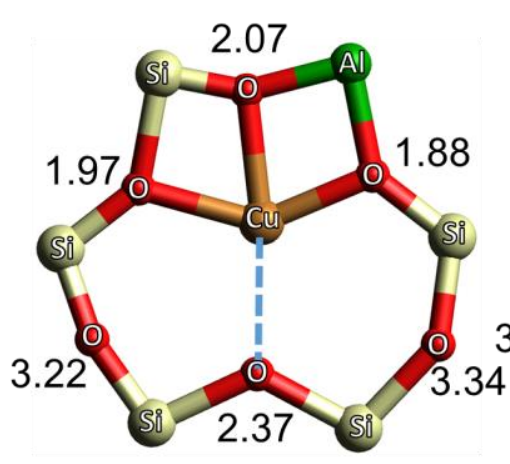

A

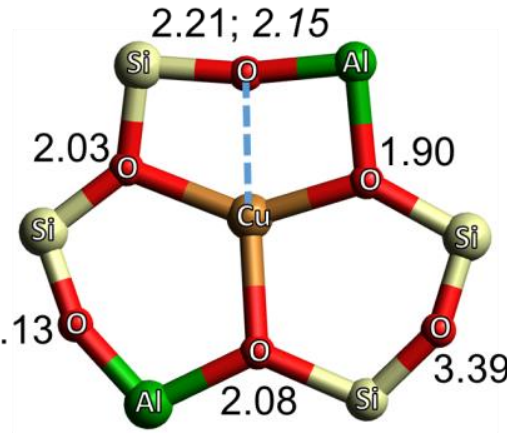

$\mathrm{D}$

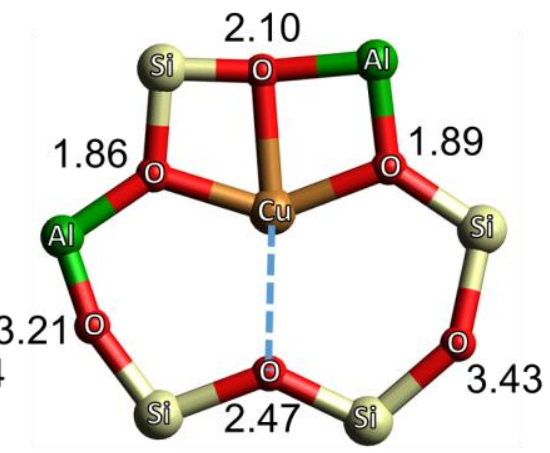

B

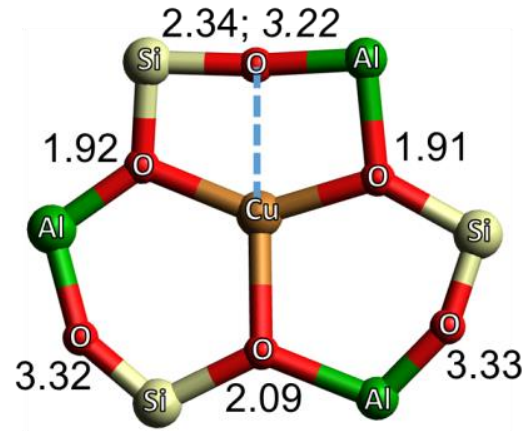

E

Figure 12. Coordination of $\mathrm{Cu}^{2+}$ in $6 \mathrm{MRs}$ of $\mathrm{FAU}$ with one (A), $2(\mathrm{~B}-\mathrm{D})$, and $3(\mathrm{E}) \mathrm{Al}$ atoms (in green), calculated by DFT. Bond distances $(\AA)$ are given. The italic distances in $D$ and $E$ are obtained by CASPT2. ${ }^{102}$

The following conclusions could be drawn: (1) Instead of a trigonal coordination, $\mathrm{Cu}^{2+}$ adopted a fourfold coordination, thus maximizing its coordination number; (2) $\mathrm{O}$ atoms of $\mathrm{Al}$ tetrahedra (Al-O-Si) are more basic than those of $\mathrm{Si}$ tetrahedra ( $\mathrm{Si}-\mathrm{O}-\mathrm{Si})$ and are preferentially taken up in the first coordination sphere; (3) As a consequence of (1) and (2), the 6MR is significantly distorted by the TMI. The geometry of the coordination complex is no longer $\mathrm{D}_{3 \mathrm{~h}}$ or $\mathrm{C}_{3 \mathrm{v}}$. (4) Therefore, the lattice is not rigid but quite flexible so as to fulfill the need of the TMI to maximize its coordination number; (5) the EPR parameters and the $d-d$ transitions depend significantly on the number and the siting of $\mathrm{Al}$ in the $6 \mathrm{MR}$. 
Table 2. CASPT2 calculated spectra $\left(\mathrm{cm}^{-1}\right)$ and g-factors for $\mathrm{Cu}^{2+}$ in $6 \mathrm{MRs}$ of FAU (Figure 12 A-E). clusters A-E represent B3LYP-DFT structures, while clusters D', E' contain a CASPT2 optimized $\mathrm{Cu}-\mathrm{O}_{B 1}$ bond.

$\begin{array}{llllllll} & \text { A } & \text { B } & \text { C } & \text { D } & \text { E } & \text { D' } & \text { E' }^{\prime} \\ \mathrm{X}^{2} \mathrm{~A}\left(\mathrm{~d}_{\mathrm{x}^{2}-\mathrm{y}^{2}}\right) & 0 & 0 & 0 & 0 & 0 & 0 & 0 \\ \mathrm{~b}^{2} \mathrm{~A}\left(\mathrm{~d}_{\mathrm{xy}}\right) & 9476 & 9334 & 7923 & 7828 & 6632 & 8411 & 7750 \\ \mathrm{c}^{2} \mathrm{~A}\left(\mathrm{~d}_{\mathrm{xz}}\right) & 10779 & 10341 & 10178 & 10141 & 9118 & 10644 & 9943 \\ \mathrm{~d}^{2} \mathrm{~A}\left(\mathrm{~d}_{\mathrm{yz}}\right) & 12348 & 12775 & 11132 & 10827 & 10501 & 11303 & 11361 \\ \mathrm{e}^{2} \mathrm{~A}\left(\mathrm{~d}_{z^{2}}\right) & 15353 & 15346 & 14776 & 14575 & 13924 & 15108 & 14850 \\ \mathrm{~g}_{z z} & 2.33 & 2.31 & 2.41 & 2.42 & 2.48 & 2.39 & 2.41 \\ \mathrm{~g}_{x x}, \mathrm{~g}_{y y} & 2.112 .04 & 2.122 .05 & 2.10 & 2.10 & 2.13 & 2.09 & 2.11 \\ & & & 2.06 & 2.06 & 2.05 & 2.06 & 2.05\end{array}$

Table 2 summarizes the energies of the ligand field excited states relative to the $d_{x^{2}-y^{2}}$ ground state of $\mathrm{Cu}^{2+}$ coordinated to the $\mathrm{O}$ atoms of the $6 \mathrm{MRs}$ found in figure $12(\mathrm{~A}-\mathrm{E})$, which can be compared to the experimental $d$ - $d$ transitions and EPR parameters for CuFAU in table 3 (along with experimental values for $\mathrm{Cu}^{2+}$ sites in other dehydrated zeolites). The calculated $g_{\|}$-values fall into two ranges: $g_{\|}=2.31-2.32$ for $6 \mathrm{MRs} A$ and $B$ and $g_{\|}=2.39-2.41$ for 6 MRs $C$, D' and E' (the latter in agreement with experiment). The calculated $d-d$ transitions fall in the experimental range (see table 3 ), but the experimental resolution is too low to resolve the two $\mathrm{Cu}^{2+}$ species. D' and E', which have CASPT2-optimized $\mathrm{Cu}-\mathrm{O}_{\mathrm{B} 1}$ bonds, were therefore chosen as models of the $\mathrm{Cu}^{2+}$ sites in FAU based on agreement with experiment. This demonstrated that the spectroscopic signatures of $\mathrm{Cu}^{2+}$ coordinated to lattice oxygens in 6MRs of zeolites is determined by the number and distribution of the Al tetrahedra in the ring. 
Table 3. Experimental EPR parameters and $d-d$ transitions of $\mathrm{Cu}^{2+}$ in dehydrated zeolites.

zeolite

EPR: $g_{\|}{ }^{a}$

EPR: $A_{\|} / \mathrm{cm}^{-1}$ a

$\mathrm{d}-\mathrm{d} / \mathrm{cm}^{-1}$

LTA

$2.37-2.41$

$(130-145) \times 10^{-4}$

$\underline{10500} ; 12200 ; 15100$

FAU

$2.36-2.41$

$(120-145) \times 10^{-4}$

10300-10700; $12600 ; 15000$

$$
2.30-2.34
$$

$(167-188) \times 10^{-4}$

MOR

2.27

$191 \times 10^{-4}$

16750

2.32

$169 \times 10^{-4}$

$\underline{13600}$

$\mathrm{CHA} \quad 2.358$

$155 \times 10^{-4}$

$\underline{10700}, \underline{12900}, \underline{14800}$

2.325

$162 \times 10^{-4}$

MFI

$$
\text { 2.26-2.28 }
$$

$180 \times 10^{-4}$

13400 (broad)

2.30-2.33

$(157-173) \times 10^{-4}$

${ }^{a} g_{\perp}$ and $A_{\perp}$ are not included because they are - within experimental error - the same for all $\mathrm{Cu}^{2+}$ species $\left(g_{\perp}=2.06\right.$ - 2.075). This is because of the low resolution of the EPR powder spectra in the perpendicular region of the spectrum; The most intense $d$-d transitions are underlined.

Conclusions drawn from Cu-bound 6MRs in FAU were found to map onto the spectroscopy of $\mathrm{Cu}^{2+}$ coordinated to $6 \mathrm{MRs}$ in MOR shown in table $3 .{ }^{99} \mathrm{Two} \mathrm{Cu}^{2+}$ species are observed with distinct $d-d$ transitions at $13500 \mathrm{~cm}^{-1}$ and $16500 \mathrm{~cm}^{-1}$. The former corresponds to a $\mathrm{Cu}^{2+}$ species with $\mathrm{g}_{\|}=2.32$; the latter to $\mathrm{C}^{2+}$ species with $\mathrm{g}_{\|}$ $=2.27 . \mathrm{Cu}^{2+}$ species with similar EPR parameters are observed for Cu-ZSM-5, however d-d bands corresponding to individual species could not be resolved. A 6MR model with one Al gives an almost perfect square planar geometry with $\mathrm{g}_{\|}=2.27$ and the $\mathrm{E}\left(\mathrm{d}_{z^{2}}\right)-$ $E\left(d_{x^{2}-y^{2}}\right)$ transition around $16500 \mathrm{~cm}^{-1}$. For the other $\mathrm{Cu}^{2+}$ species, a $6 \mathrm{MR}$ with 2 Al gives agreement between theoretical and experimental data, i.e. a $d$-d band maximum around $13500 \mathrm{~cm}^{-1}$ and $\mathrm{g} \|=2.32$, again demonstrating that the Al T-sites within a $6 \mathrm{MR}$ have a significant impact on the electronic structure of the resulting metal-bound site. 
The above results from spectroscopy and calculations enabled quantitative comparison of the ligand fields provided by $6 \mathrm{MRs}$ in MOR and FAU. For these lattices, the ligand field excited states of $\mathrm{Cu}^{2+}$ in the $6 \mathrm{MRs}$ are characteristic of sites with nearly square planar geometry. This is shown by the small difference between the $d_{x z}$ and $d_{y z}$ states, which are degenerate in rigorous D4h symmetry. (As shown in table 2, this difference is in the range $400-600 \mathrm{~cm}^{-1}$ for MOR ${ }^{99}$ and $2400-650 \mathrm{~cm}^{-1}$ for $6 \mathrm{MRs}$ of FAU). The ligand field strength of square planar sites is expressed by $10 \mathrm{Dq}=\left(E\left(d_{x y}\right)-\right.$ $\left.E\left(d_{x^{2}-y^{2}}\right)\right) .10 D q$ values and average Cu-O distances are summarized in Table 4.

Table 4. $10 \mathrm{Dq}$ values and average $\mathrm{Cu}-\mathrm{O}$ distances of $\mathrm{Cu}^{2+}$ in various six-membered rings of FAU (figure 12) and MOR (figure 10). ${ }^{99}$

$\begin{array}{llll}\text { Sites of FAU } & \text { Number of Al } & \text { 10Dq }\left(\mathbf{c m}^{-1}\right) & \text { Average Cu-O (nm) } \\ \text { A } & 1 & 9476 & 0.2073 \\ \text { B } & 2 & 9334 & 0.2080 \\ \text { C } & 2 & 7923 & 0.2045 \\ \text { D } & 2 & 7828 & 0.2055 \\ \text { D' } & 2 & 8411 & 0.2040 \\ \text { E } & 3 & 6632 & 0.2064 \\ \text { E' } & 3 & 7750 & 0.2035\end{array}$

\section{Sites of MOR}

$\begin{array}{llll}\boldsymbol{\alpha}, \mathbf{Y} & 1 & 11020-11623 & 0.1980-0.2000 \\ \boldsymbol{\alpha} & 2 & 10400-10600 & 0.2055\end{array}$

From table 4, the $6 \mathrm{MRs}$ of MOR are associated with stronger ligand fields than in 6MRs of FAU. This is in line with the observation that the average $\mathrm{Cu}-\mathrm{O}$ distance is also shorter in 6MRs of MOR than in 6MRs of FAU. In both cases the ligand field strength decreases with increasing number of $\mathrm{Al}$ tetrahedra in the $6 \mathrm{MRs}$, although the average 
Cu-O distance also decreases. One would have expected the reverse, as $\mathrm{Cu}^{2+}$ preferentially coordinates to the $\mathrm{O}$ atoms of the $\mathrm{Al}$ tetrahedra and these $\mathrm{O}$ atoms are more basic than the $\mathrm{O}$ atoms bridging two $\mathrm{Si}$ atoms (Si-O-Si). ${ }^{103-105}$ However, this is counteracted by distortions away from square planar geometry. Deviations of O-M-O angles from 90 degrees, lone pairs on the lattice $O$ ligands becoming misaligned with the lobes of $d_{x^{2}-y^{2}}$ (misdirected valence) and out-of-plane distortions of the Fe atom could all contribute to a diminished $d_{x^{2}-y^{2}} / d_{x y}$ splitting. 
3. Active Site Spectroscopic Probes. In bioinorganic chemistry, many spectroscopic techniques have been developed to focus on metal active sites in a dominant protein matrix. The goal in metallozeolite chemistry is similar: to focus on a metal active site within a dominant aluminosilicate matrix. A critical difference here is the presence of spectator metal centers. These can be difficult (or impossible) to distinguish from active sites using bulk techniques such as EXAFS and magnetic susceptibility. On the other hand, methods with single-site resolution (e.g. diffuse reflectance UV-vis, magnetic circular dichroism, electron paramagnetic resonance, and Mössbauer spectroscopy vide infra) enable correlation of spectroscopic features to individual metal centers. This is often accomplished by tracking spectral changes associated with reactivity. Active site spectroscopic handles can then be probed selectively using e.g. resonance Raman spectroscopy and variable-temperature variable-field magnetic circular dichroism spectroscopy (VTVH-MCD). This section provides an overview of the methods and associated experimental strategy used to elucidate $\mathrm{Fe}$ and $\mathrm{Cu}$ active sites in zeolites and their reactive metal-oxo intermediates. For most of the studies covered in this review, these techniques are applied to samples in the gas phase that operate under mild conditions (i.e. gas-solid operation). In most cases, their extension to catalysts operating in the liquid-solid or gas-liquid-solid regime is straightforward. For systems that operate at elevated temperatures and pressures, a current challenge is developing in situ or 'operando' techniques to probe active sites that may only exist under operating conditions. ${ }^{106}$ 


\section{1 - Ground and Excited State}

Properties of Transition Metal Sites. Spectroscopic methods spanning ten decades of photon energy are currently used to probe ground- and excited-state properties of transition metal complexes (e.g. for $\mathrm{CuCl}_{4}^{2-}$ in figure 13). At low energy, microwave photons are used to drive transitions between ground state sublevels split in the presence of a magnetic field, providing insight into molecular magnetism (see 3.1.1). Moving up in energy, NIR-Vis-UV photons excite electrons among valence levels, resulting in ligand field and charge transfer transitions, which encode information related to metal-

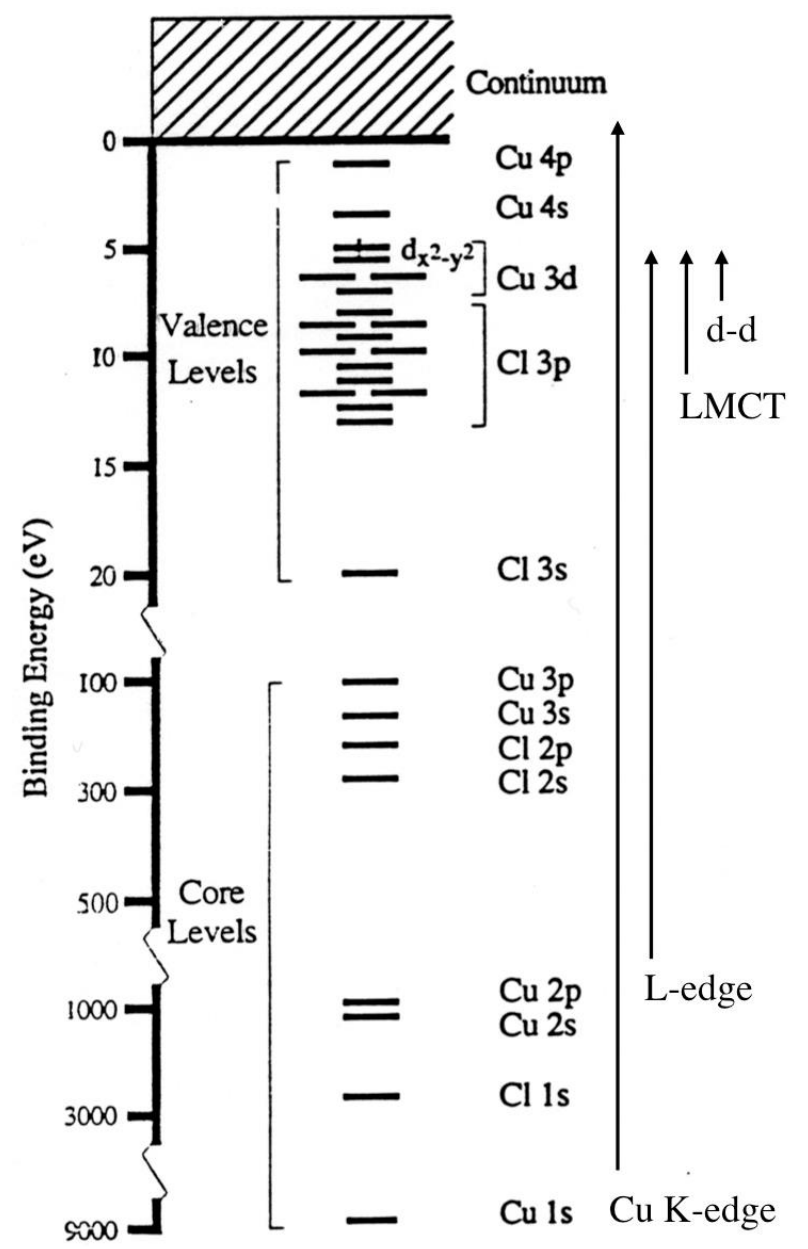

Figure 13. Electronic structure of $\mathrm{D}_{4 \mathrm{~h}}\left[\mathrm{CuCl}_{4}\right]^{2-}$, showing excited state spectroscopic transitions ranging from 1-10,000 eV. Ground state methods probe transitions on the order of $0.0001 \mathrm{eV}$ (not shown).

ligand bonding (see 3.1.2, 3.1.3). X-ray photons excite electrons from core levels to valence levels and into the continuum. (While core excitations provide extensive chemical insight, XAS does not directly provide the single-site resolution required for metallozeolite chemistry.) Finally, at still higher photon energies $(\gamma)$, it is possible to probe nuclear excited states through resonant (Mössbauer spectroscopy - 3.4.2) and non-resonant absorption methods (NRVS - 3.4.3). 
3.1.1 - Paramagnetic Ground States. The ground state of a high spin transition metal center with $n$ unpaired $d$ electrons will have a total spin $S=n / 2$, resulting in an effective magnetic moment $\mu=g_{\mathrm{e}} \beta(\mathrm{S}(\mathrm{S}+1))^{1 / 2}$ in the absence of spin-orbit coupling (SOC), where $g_{\mathrm{e}}=2.0023$ is the free electron $g$-value and $\beta$ is the Bohr magneton. This magnetic moment can be probed using a number of techniques (e.g. magnetic susceptibility, EPR, and VTVH-MCD - see 3.3.3, 3.4.1). Its magnitude reflects the spin state of the metal, and this provides key insight into the metal center's oxidation state and nuclearity (vide infra).

The magnetism of transition metal ions encodes additional information arising from the spin-orbit interaction - a relativistic effect that couples electron spin and orbital angular momenta. SOC effects on ground state magnetism reflect orbital properties that include ligand field splittings and metal-ligand covalency. These effects can be determined experimentally, and then related to molecular electronic structure using ligand field theory. This is generally accomplished using the spin Hamiltonian formalism - a phenomenological description of ground state spectral features arising in EPR (3.4.1) and VTVH-MCD (3.3.3). The spin Hamiltonian for an effective spin-1/2 system is given in equation 1 :

$$
\hat{\mathrm{H}}=\beta \vec{H} \cdot \tilde{g} \cdot \vec{S}+\vec{l}^{M} \cdot \tilde{A}^{M} \cdot \vec{S}+\sum_{L} \vec{l}^{L} \cdot \tilde{A}^{L} \cdot \vec{S}
$$

This Hamiltonian describes the Zeeman splitting of an $S=1 / 2$ system in an external magnetic field $\vec{H}$, illustrated in figure 14 (including nuclear spin $I=1 / 2$ ). In this expression, $\beta$ is the Bohr magneton, $\tilde{g}$ is the molecular $g$ tensor, $\vec{S}$ is the effective electron spin operator, $\vec{I}^{M}$ is the nuclear spin operator of the metal, $\tilde{A}^{M}$ is the metal 
hyperfine tensor, $\vec{I}^{L}$ is the nuclear spin operator of ligand $L$, and $\tilde{A}^{L}$ is the hyperfine tensor of ligand $L$. Molecular $g$-values (components of the diagonalized $g$-tensor) can be measured

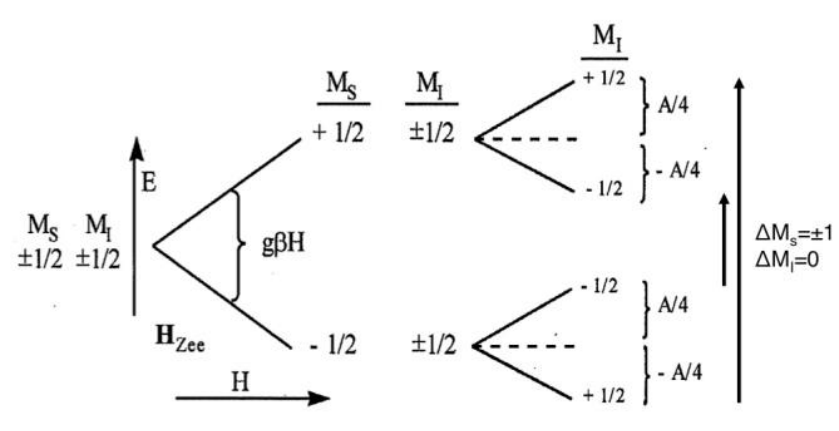
Electron Zeeman

Hyperfine

EPR

using a number of techniques, including Figure 14. Effects of Zeeman splitting (left, center) and hyperfine coupling $A$ (right) on a system with EPR (3.4.1), VTVH-MCD (3.4.3), and electron spin $\mathrm{S}=1 / 2$ and nuclear spin $\mathrm{I}=1 / 2$.

magnetic susceptibility. For transition metal ions, these may deviate significantly from the free electron $g$ value of 2.0023 due to SOC with low-lying ligand field excited states. These deviations can be related to ligand field splittings and metal-ligand covalencies using equations from ligand field theory. ${ }^{107-111}$

For systems with non-zero nuclear spin (either on the metal or ligands), the ground state will undergo additional smaller splittings due to the hyperfine interaction between the nuclear spin I and the electron spin $S$ (figure 14). Metal hyperfine coupling (described by the second term in equation 1) can be resolved in EPR, where it splits resonances into $2 /+1$ evenly spaced lines separated by an amount determined by the components of the metal hyperfine tensor $\tilde{A}^{M}$ (see 3.4.1 for detail). There are three contributions to $\tilde{A}^{M}$, each reflecting the nature of the ground state and its covalency. ${ }^{108,110,111}$ For a metal complex with unpaired $d$ electrons, the core $2 s$ and $3 s$ electrons are spin polarized through s/d exchange, resulting in net negative spin density at the nucleus. This leads to an indirect isotropic Fermi contact interaction between the nuclear spin and the electron spin density at the nucleus, given as

$$
A_{\text {Fermi }}=\frac{8 \pi}{3} g_{e} \beta_{e} g_{n} \beta_{n}\left[\left|\Psi_{\uparrow}(0)\right|^{2}-\left|\Psi_{\downarrow}(0)\right|^{2}\right]
$$


where $g_{\mathrm{e}}$ is the free electron $\mathrm{g}$-value, $g_{\mathrm{n}}$ is the nuclear $\mathrm{g}$ value, $\beta_{\mathrm{e}}$ is the electron (Bohr) magneton, $\beta_{\mathrm{n}}$ is the nuclear magneton, and the bracketed term is the net spin density at the nucleus. Covalent delocalization of valence $d$ electrons decreases $\mathrm{s} / \mathrm{d}$ exchange, resulting in a smaller Fermi contact term. Anisotropic contributions to $\tilde{A}^{M}$ arise from spin-dipolar interactions between the spin magnetic moments of the electron and nucleus:

$$
A_{\text {spin-dipolar }}=\frac{g_{e} \beta_{e} g_{n} \beta_{n}}{\left\langle r^{3}\right\rangle} \frac{1}{2}\left\langle 3 \cos ^{2} \theta_{e}-1\right\rangle\left(3 \cos ^{2} \theta_{n}-1\right)
$$

In this expression, $r$ is the radial position of the electron, $\theta_{\mathrm{e}}$ is the angular position of the electron with respect to the molecular $z$ axis, and $\theta_{n}$ is the angle between the nuclear spin (oriented along $\vec{H}$ ) and the molecular $z$ axis. The expectation values in angled brackets are evaluated over the shape of the occupied $d$ orbital. Since the spin-dipolar interaction is localized on the metal, this contribution also decreases with covalent delocalization. A second anisotropic contribution arises from the dipolar interaction between the orbital magnetic moment of the electron and the spin magnetic moment of the nucleus. This interaction is described by the orbital-dipolar ter in the spin Hamiltonian:

$$
\widehat{H}_{\text {orbital-dipolar }}=\frac{g_{e} \beta_{e} g_{n} \beta_{n}}{\left\langle r^{3}\right\rangle}\left[\vec{L} \cdot \vec{I}-\frac{3}{2} \xi[(\vec{L} \cdot \vec{S})(\vec{L} \cdot \vec{I})+(\vec{L} \cdot \vec{I})(\vec{L} \cdot \vec{S})]\right]
$$

where $\xi=(2 \ell+1-4 S) /[S(2 \ell-1)(2 \ell+3)(2 \mathrm{~L}-1)]$. The orbital-dipolar interaction therefore reflects spin-orbit coupling to ligand field excited states, evident from the ground state gvalues deviating from 2.0023 (see 3.4.1 for detail). 
Superhyperfine coupling, described by the third term in equation 1, arises mostly from delocalization of the electron spin over the nuclear spin of the ligands. As with metal hyperfine coupling, the hyperfine tensor of ligand $L\left(\tilde{A}^{L}\right)$ includes contributions from Fermi contact and magnetic dipolar interactions. These interactions are weak relative to the hyperfine interaction with the metal. They can often be resolved using EPR, or with higher resolution experiments such as electron nuclear double resonance (ENDOR) and pulsed EPR via electron spin echo envelope modulation (ESEEM). ${ }^{112}$

For systems with more than one unpaired electron $(S \geq 1)$, the $2 S+1$ components the ground state will generally be split in the absence of a magnetic field. ${ }^{108,109,111}$ This zero-field splitting (ZFS) is described by an additional term in the spin Hamiltonian:

$$
H_{Z F S}=D\left(\widehat{S}_{Z}^{2}-\mathrm{S}(\mathrm{S}+1) / 3\right)+E\left(\widehat{\mathrm{S}}_{X}^{2}-\widehat{\mathrm{S}}_{y}^{2}\right)
$$

where $D$ and $E$ are the axial and rhombic ZFS parameters, respectively. The effects of axial and rhombic ZFS are different for Kramers (odd number of electrons, 1/2-integer spin) and non-Kramers systems (even number of electrons, integer spin). For Kramers ions, axial ZFS $(D \neq 0)$ splits the spin manifold into $\pm M_{s}$ pair states known as Kramers doublets (figure 15, left). Rhombic ZFS $(E \neq 0)$ mixes $M_{s}$ states with $\Delta M_{s}= \pm 2$. By doublegroup symmetry, the two components of a Kramers doublet are affected identically by this mixing, and they remain degenerate in the absence of an external magnetic field. For non-Kramers ions, axial ZFS separates the spin manifold into a singly-degenerate $M_{s}=0$ state along with $\pm M_{s}$ pair states known as non-Kramers doublets (figure 15 , right). 
As before, rhombic ZFS mixes $M_{s}$ states with $\Delta M_{s}= \pm 2$, but this has an additional effect on a non-Kramers ion. In this case, this mixes and splits the $M_{s}= \pm 1$ doublet by the amount $\mathrm{S}(\mathrm{S}+1)|E| \quad(6|E|$ in figure 15). For nonKramers ions with $S \geq 2$, the $M_{s}= \pm 2, \pm 3, \pm 4 \ldots$ doublets will also be split, but by incrementally smaller amounts due to higherorder rhombic ZFS (e.g. $\delta$ in figure 15). As a result, the ground state of a non-Kramers

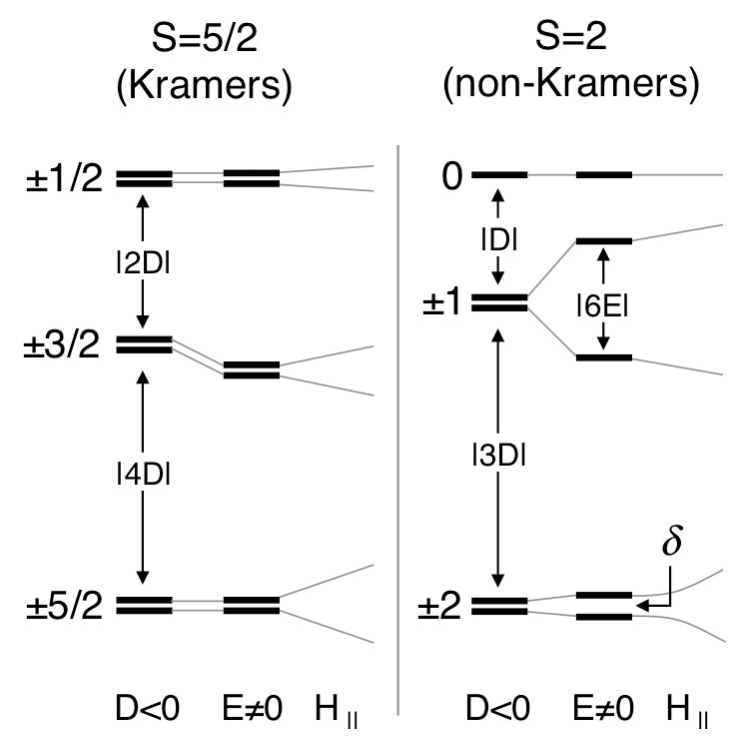
system will be non-degenerate even in the absence of an external magnetic field (except in the special case where $\mathrm{D} \leq 0, \mathrm{E}=0$ ).

For transition metal ions, SOC provides the dominant mechanism for ZFS. $D$ and $E$ therefore contain information about the ground state electronic wavefunction, including contributions from low-lying excited states with $\Delta S=0, \pm 1$. Zero-field splittings can be measured directly using EPR, or inferred from the combined effects of temperature and magnetic field on magnetization (via SQUID magnetic susceptibility or VTVH-MCD). ${ }^{19,113}$ For some techniques - EPR in particular - different approaches are required for Kramers versus non-Kramers ions (see 3.4.1). ${ }^{108,110}$ Experimental values of $D$ and $E$ can then be correlated to ligand field splittings reflecting coordination geometry and covalency using ligand field theory. For systems with two or more metal centers coupled by bridging ligands, additional terms describing exchange coupling must be 
incorporated into the spin Hamiltonian (including isotropic, anisotropic, and antisymmetric exchange). ${ }^{114,115}$

3.1.2 - Ligand Field Excited States. Moving up in energy from the ground state to the NIR-vis spectral region, the first electronic excited states of a transition metal complex are generally ligand field transitions, which involve excitations of electrons between valence molecular orbitals with dominantly metal $d$ character. Ligand field transitions can be challenging spectroscopic targets, as they are parity (Laporte) forbidden and therefore weak in absorption $\left(\varepsilon<100 \mathrm{~mol}^{-1} \mathrm{~cm}^{-1}\right)$. Techniques such as CD (for chiral sites) and MCD (for all sites, see 3.3) have different selection rules, and ligand field transitions can be observed with considerable intensity in these experiments, particularly in low-temperature MCD (see 3.3.2). ${ }^{19,116}$

Since the $d$ orbitals participate directly in metal-ligand bonding, ligand field transitions are highly sensitive to coordination geometry and ligand identity, and correlate to each in predictable ways as described by ligand field theory (vide infra). Coordination geometries that may be encountered in metallozeolite and metalloenzyme chemistry are shown in figure 16, along with characteristic $d-$ orbital splittings. These reflect differences in $\sigma$ versus $\pi$ bonding interactions with ligands. For an octahedral metal center with $\sigma / \pi$ donor ligands (figure 16a), the d orbitals split
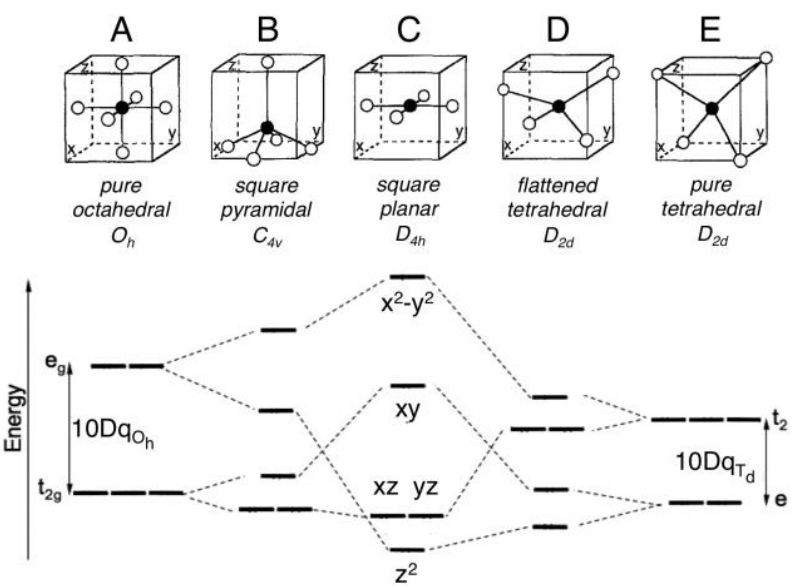

Figure 16. Five commonly-encountered coordination geometries for transition metal complexes (top), and their associated ligand field splittings (bottom). 
into a triply-degenerate $t_{2 g}$ set and a doubly-degenerate $e_{g}$ set at higher energy separated by the amount 10Dqon. Removing one axial ligand results in a square pyramidal site (figure 16b). This removes a strongly destabilizing $\sigma$-antibonding interaction with $d_{z^{2}}$ and weaker $\pi$-antibonding interaction with $d_{x z / y z}$. To compensate for axial ligand loss, the equatorial ligands contract, destabilizing $d_{x^{2}-y^{2}}$ and $d_{x y}$. Removing the second axial ligand results in a square planar site (figure 16c). A key feature of square planar geometry is the large energetic separation between $d_{z^{2}}$ and $d_{x^{2}-y^{2}}$. This is a consequence of a strong $\sigma$-antibonding interaction with the ligands (the lobes of $d_{x^{2}-y^{2}}$ are directed along the M-L bonds), as well as the unique stability of $d_{z^{2}}$ in square planar geometry due to $4 s$ mixing in the absence of axial ligands. The effects of a $D_{2 d}$ distortion toward tetrahedral geometry - bringing one trans pair of ligands above the molecular plane and one pair below the plane - is shown in figure $16 \mathrm{~d}$. This eventually leads to a tetrahedral structure (figure $16 \mathrm{e}$ ), with a t2 level above an e level separated by the amount $10 \mathrm{Dq} \mathrm{Td}_{\mathrm{d}}=-4 / 9(10 \mathrm{Dqoh})$. This distortion toward tetrahedral geometry has been particularly significant in bioinorganic chemistry due to its contribution to the properties of the type 1 "blue copper" active sites (see 6.3 .3 for detailed discussion). ${ }^{117,118}$ 
3.1.3 - Charge Transfer Excited States. Moving to higher energy, the next electronic excited states for first row transition metal centers are charge transfer transitions. For first row metals in zeolites, these are typically ligand to metal charge transfer (LMCT) transitions, which involve excitation of electrons from filled ligand-based valence molecular orbitals into unoccupied or halfoccupied metal d orbitals (figure 17A). Unlike ligand field transitions, LMCT transitions are electric dipole allowed, and can be intense in Abs spectroscopy $\left(\varepsilon>1000 \mathrm{~mol}^{-1} \mathrm{~cm}^{-1}\right)$. They may also be strongly coupled to metal-ligand vibrations through excited state distortions, as LMCT transitions involve a large change in bonding moving from the ground state to the excited state. This effect is probed using resonance Raman spectroscopy (see 3.2).
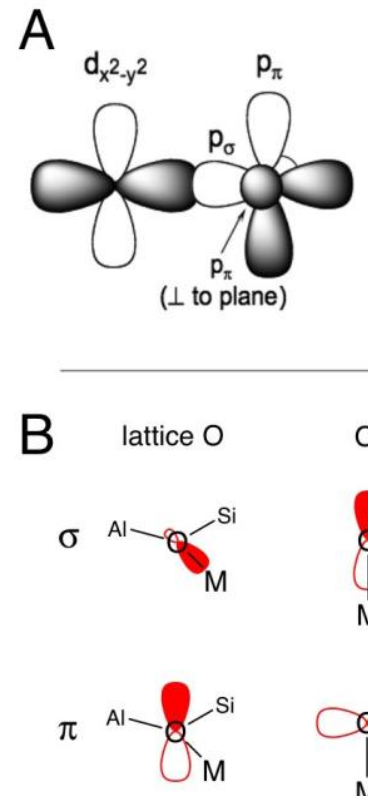

Figure 17. (A) The difference in energy and overlap between empty metal $d$ and filled ligand valence orbitals leads to weak low- energy $\pi$ and intense high-energy $\sigma$ charge transfer transitions. (B) Metal-oxygen bonding interactions encountered in metallozeolite chemistry. For $\mathrm{O}_{2}$-derived ligands (right), there are two perpendicular $\pi^{*}$ orbitals that can participate in pseudo- $\sigma$ (top) and $\pi$ interactions (bottom), as well as an empty $\sigma^{*}$ orbital that can

\section{LMCT transitions occur between participate in $\sigma$ backbonding.}

orbitals directly involved in metal-ligand bonding. They therefore contain information about donor/acceptor orbital overlap and covalency. For ligands commonly encountered in metallozeolite chemistry (figure 17b), there are both $\sigma$ and $\pi$ lone pairs available for bonding. Zeolite lattice $\mathrm{O}$ atoms, which bridge adjacent $\mathrm{Al}$ and $\mathrm{Si} \mathrm{T}$-sites, have an in-plane $\sigma$ hybrid lone pair and an out-of-plane $p_{\pi}$ lone pair available for bonding. The in-plane $p$ orbital participates in bonding with the T-sites, and is not 
available as a donor orbital. Oxo ligands (derived from $\mathrm{O}_{2}$ or $\mathrm{N}_{2} \mathrm{O}$ ) may bind in a bridging or terminal fashion, with donor interactions from three filled p orbitals (one $\sigma$, two $\pi$ ). For $\mathrm{O}_{2}$-derived ligands (superoxide, peroxide), there is a lone pair available for pseudo- $\sigma$ donation derived from the filled $\pi^{*}$ orbital, a perpendicular $\pi^{*}$ orbital (filled or half-filled) available for $\pi$ donation, and an empty $\sigma^{*}$ orbital available for backbonding from the metal and for electrophilic reactivity. Since $\sigma$ bonding is stronger than $\pi$ bonding due to directional orbital overlap, $\sigma$ lone pairs will be stabilized relative to the $\pi$ lone pair(s) through bonding with the metal (figure 17b). $\sigma$-LMCT transitions therefore occur at high energy relative to $\pi$-LMCT bands, and because LMCT intensity is proportional to donor/acceptor orbital overlap, $\sigma$-LMCT transitions are generally more intense. ${ }^{119}$

Bonding interactions are dominated by occupied ligand donor orbitals and unoccupied metal valence orbitals that are close in energy and have significant overlap. Thus highly covalent ligand-metal bonds exhibit intense, low energy LMCT transitions. These are often associated with ligands having high energy lone pairs (e.g. oxides) and metals with low energy $d$ orbitals (high $Z_{\text {eff }}$ metals). Relevant to this review are metaloxygen intermediates, where covalent bonding leads to intense, low energy oxygen $\rightarrow$ metal CT transitions. (This is in contrast to ionic complexes, which exhibit weak CT transitions at higher energy, reflecting low covalency.) For these intermediates, charge transfer spectroscopy provides a direct connection between electronic structure and function, as metal-oxygen covalency contributes significantly to reactivity (see sections $4-6)$. 
3.2 - Resonance Raman Spectroscopy. Like Raman spectroscopy, resonance Raman $(\mathrm{rR})$ spectroscopy is used to study molecular vibrations excited through inelastic scattering of laser photons. ${ }^{107,119} \mathrm{In} \mathrm{rR}$, the photon energy is tuned to match an intense absorption band associated with a transition to an electronic excited state, resulting in resonant enhanced vibrational scattering. Depending on the specific structural and electronic properties of the excited state, this leads to resonant enhancement of certain vibrations by up to $3-4$ orders of magnitude. From an experimental standpoint, this offers two key advantages. First, resonantly enhanced features of an active site are intense and easily resolved above background non-resonant contributions. Second, resonant enhancement is selective for vibrations coupled to a particular absorption feature, making $\mathrm{rR}$ a site-selective technique. This is particularly important in metallozeolite chemistry, where $\mathrm{rR}$ can be used to resolve the vibrations of a minority species that exists in a heterogeneous distribution of metal sites.

The dominant mechanism for resonant Raman scattering, known as $A$ term enhancement for an electric dipole allowed transition, derives from the first term in the Kramers-Heisenberg-Dirac dispersion equation for the Raman polarizability tensor, shown in equation 6. ${ }^{120}$ (Other intensity mechanisms exist, but these reflect vibronic coupling and are generally less important in metallozeolite and non-heme metalloenzyme spectroscopy). ${ }^{119}$

$$
A^{\rho \sigma}=\sum_{e v} \frac{\left\langle\Psi_{g}\left|\hat{M}_{\rho}\right| \Psi_{e}\right\rangle\left\langle\chi_{g f} \mid \chi_{e v}\right\rangle\left\langle\Psi_{e}\left|\hat{M}_{\sigma}\right| \Psi_{g}\right\rangle\left\langle\chi_{e v} \mid \chi_{g i}\right\rangle}{v_{e v}-v_{g i}-v_{L}+i \Gamma_{e v}}
$$


In this expression, $\rho, \sigma$ are Cartesian coordinates $(\mathrm{x}, \mathrm{y}, \mathrm{z}), M_{\rho}, M_{\sigma}$ are components of the electric dipole operator, $\Psi_{g}, \Psi_{e}$ are the ground and excited state electronic wavefunctions (respectively), $\chi_{g i}, \chi_{g f}$ are the initial and final ground state vibrational wavefunctions, and $\chi_{\mathrm{ev}}$ is the excited state vibrational wavefunction. In the denominator, $v_{\mathrm{gi}}, v_{\mathrm{gf}}, v_{\mathrm{ev}}$ are the initial/final frequencies (energies) of the ground state and excited state, while $V L$ is the photon frequency. $\Gamma_{\mathrm{ev}}$ is a damping factor inversely proportional to the excited state lifetime. Note that $A^{\rho \sigma}$ in equation 6 becomes very large when the photon energy matches the allowed excited state absorption energy, and it is this behavior in the energetic vicinity of an intense absorption band that leads to resonant enhancement.

From this expression, which includes the square of electric dipole operator, $A$ term enhancement $\left(\propto A^{2}\right)$ of vibrational mode $\chi$ is proportional to the square of the intensity of the electronic absorption band. It is also proportional to the square of the overlap between the ground and excited state vibrational wavefunctions. For this to be non-

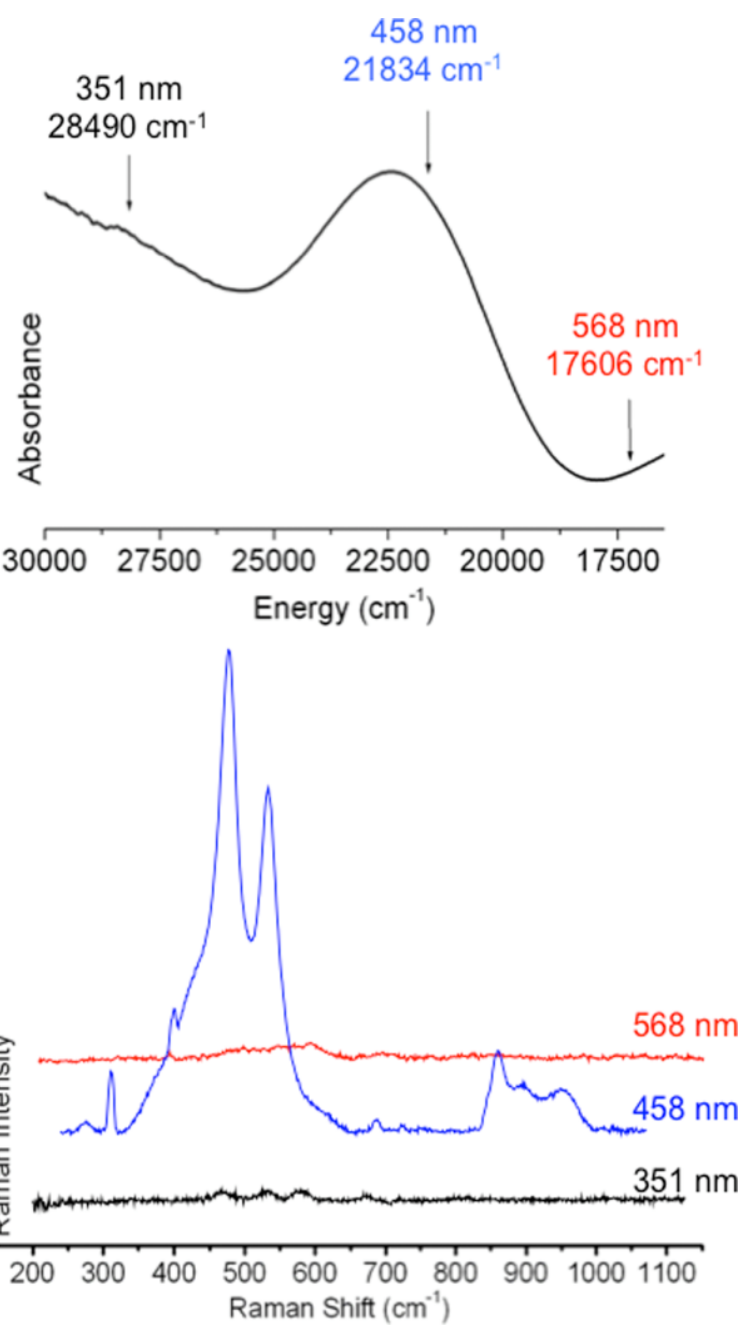

Figure 18. Effect of excitation energy on resonance intensity (bottom), which is maximized for photons tuned to an absorption feature (458 nm, top). Adapted with permission from reference ${ }^{18}$. Copyright 2014 American Chemical Society. 
zero, there must be a geometric distortion in the excited state relative to the ground state along the $\chi$ normal mode, and since excited state distortions can only occur along totally symmetric normal modes of vibration, only totally symmetric vibrations experience $A$ term enhancement. ${ }^{120}$ The energy dependence of this integral follows the band shape since they have the same origin (figure 18).

$\mathrm{rR}$ spectroscopy is often used to probe charge transfer transitions, as these are associated with high absorption intensities and large distortions of the ligand-metal bond involved in the charge transfer transition. This leads to efficient $A$ term enhancement of these ligand-metal vibrations. Large excited state distortions typically derive from weaker $M-L$ bonding in the excited state, leading to $M-L$ elongation. This can be used to assign charge transfer transitions to a specific L-M bond, including the $\sigma$ (large ES distortion) versus $\pi$ (smaller ES distortion) contributions to the bonding interaction. ${ }^{121}$

3.3 - Magnetic Circular Dichroism Spectroscopy. Magnetic circular dichroism spectroscopy (MCD) involves the induction of circular dichroism by the presence of a longitudinal magnetic field ( $\mathrm{H}$ parallel to the propagation of the circularly polarized light). This experiment probes Zeeman splittings of paramagnetic ground and excited states, as well as magnetic field-induced mixing between states. From the general expression for MCD intensity (equation 7), there are three contributions by which an MCD transition can gain intensity. ${ }^{122-125}$

$$
\frac{\Delta A}{E}=\text { const }: \beta H\left[A_{1}\left(\frac{\partial f(E)}{\partial E}\right)+\left(B_{0}+\frac{C_{0}}{k_{B} T}\right) f(E)\right]
$$


In this expression, $\Delta \mathrm{A}$ is the difference in absorption of left versus right circularly polarized light of energy $E=h v$ in the presence of a magnetic field of magnitude $H . f(E)$ is the absorption band shape, and $\partial f(\mathrm{E}) / \partial \mathrm{E}$ its first derivative. The constants $\mathcal{A}_{1}, \mathcal{B}_{0}$, and $C_{d} / \mathrm{k}_{\mathrm{B}} \mathrm{T}$ quantify the magnitude of MCD intensity arising from the linear $\mathcal{A}-, \mathcal{B}$-, and $C$-term mechanisms, described below. $\mathcal{A}_{1}$ is the contribution of any derivative-shaped contribution to MCD intensity, $\mathscr{B}_{0}$ is the contribution of any temperature-independent feature with an absorption bandshape, and $C_{0}$ is the contribution of any feature with the absorption bandshape where the magnitude increases in intensity with decreasing temperature (as 1/T). MCD studies generally focus on paramagnetic metal centers and measure $C$-term intensity, as this dominates at low temperature and has rich information content. ${ }^{116}$ MCD intensity also correlates to a specific chromophore, and it can therefore be site-selective in heterogeneous systems. ${ }^{35}$ 
3.3.1 - $\mathcal{A}$ - and $\mathcal{B}$-term mechanisms. For

a non-degenerate ground state and a degenerate electronic excited state (figure 19a) in the absence of a magnetic field, LCP and RCP transitions occur at
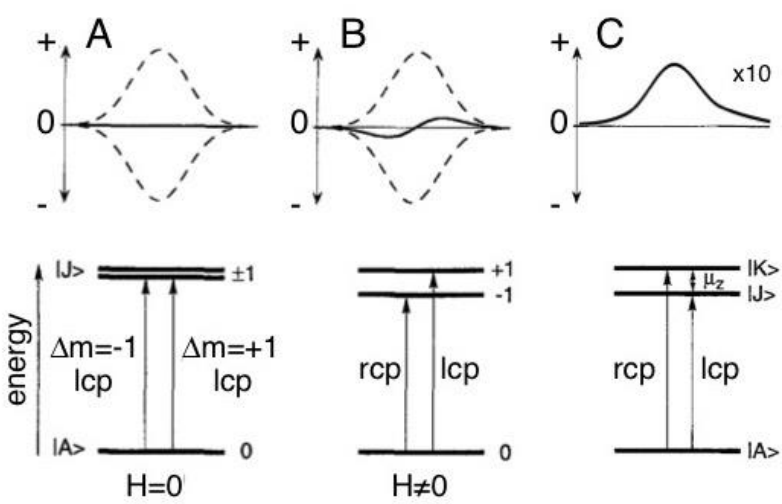

the same energy with equal and opposite

Figure 19. $\quad M C D \quad \mathcal{A}$-term $(A, B)$ and $\mathcal{B}$-term mechanisms (C, with 10x intensity scale).

magnitude, and no net MCD intensity is

observed. In the presence of a magnetic field (figure 19b), the excited state experiences a Zeeman splitting determined by its $g$ values. If the excited state $g$ value is non-zero (i.e. for states carrying angular momentum), LCP and RCP transitions from the ground state occur with equal and opposite magnitude, but at different energies (split on the order of $10 \mathrm{~cm}^{-1}$ for a $6 \mathrm{~T}$ magnetic field). The net effect is in a temperature independent, derivative shaped feature known as an $\mathcal{A}$-term. $\mathfrak{B}$-term intensity has a similar origin, but in this case the MCD signal arises through field-induced mixing between non-degenerate states (states $J$ and $K$ in figure 19c). The net effect is a temperature independent, absorption shaped feature known as a $\mathfrak{B}$-term. If the fieldinduced mixing occurs exclusively between the two excited states, the result is a pair of B-terms with equal magnitude and opposite sign known as a (temperature-independent) pseudo $\mathcal{A}$-term. Note that temperature-dependent $\mathfrak{B}$-term intensity can arise when field induced mixing occurs between the ground state and a thermally accessible low-lying excited state (vide infra). 
3.3.2 - The c-term mechanism. For systems with degenerate ground states, in particular paramagnetic metal centers, temperature-dependent MCD intensity will generally be observed. ${ }^{116,122,123}$ In the absence of a magnetic field, LCP and RCP transitions from a degenerate ground state occur at the same energy with equal and opposite magnitude, resulting in no net MCD intensity (figure 20a). In the presence of a magnetic field, the ground state experiences a Zeeman splitting determined by the ground state's $g$ values. LCP and RCP transitions occur at with equal and opposite magnitude, but at different energies (split by $g \beta H)$. At high temperature $\left(\mathrm{k}_{\mathrm{B}} \mathrm{T}>>g \beta H\right)$, the components of the ground state are equally Boltzmann-populated, and the net effect is a weak derivative-shaped feature (an $\mathcal{A}$-term, figure 20b). At low temperature $\left(\mathrm{k}_{\mathrm{B}} \mathrm{T} \sim g \beta \mathrm{H}\right)$, the lowest Zeeman-split component of the ground state becomes preferentially Boltzmann-populated, resulting in a temperature-dependent, absorption shaped MCD feature called a $C$-term (figure 20c). $C$-term intensity generally dominates at low temperature (by a factor of 100-1000). This is evident from equation 7 , which
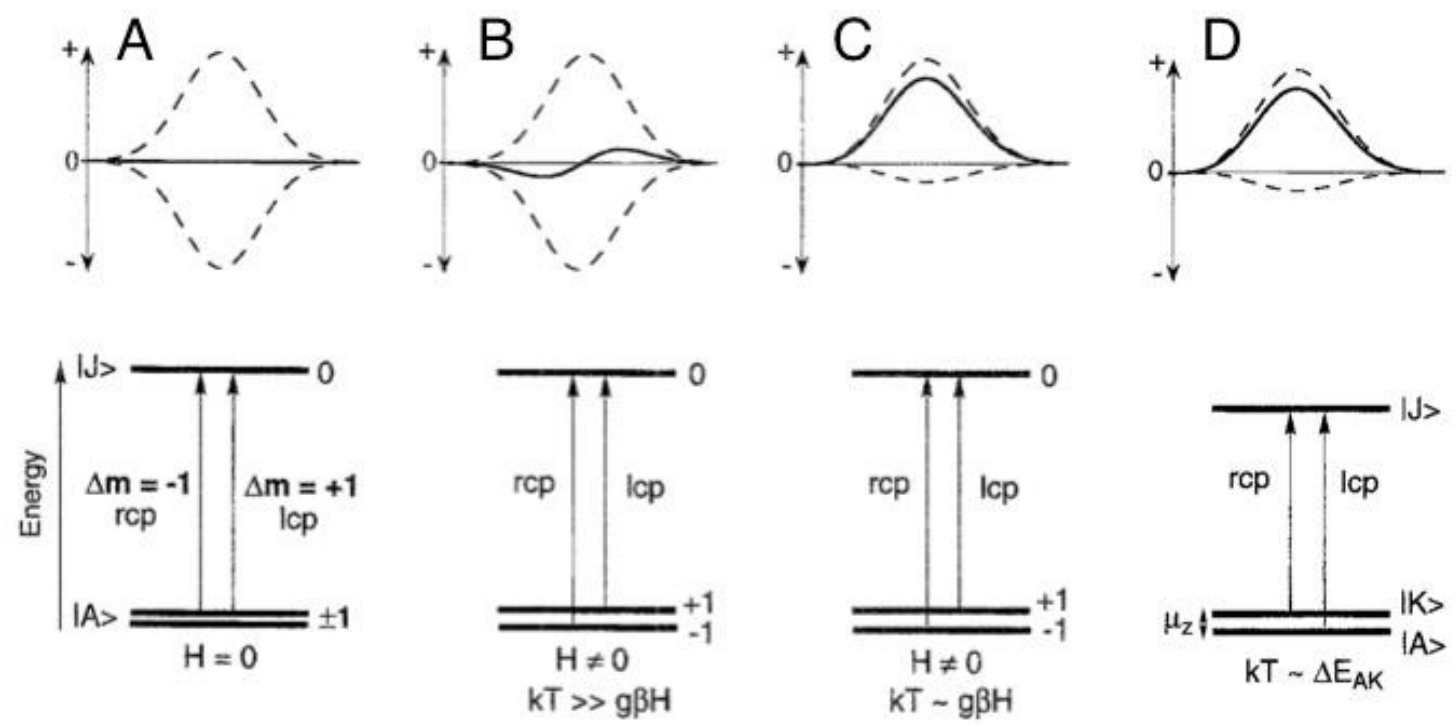

Figure 20. MCD $c$-term $(A-C)$ and temperature-dependent $\boldsymbol{B}$-term mechanisms (D). 
shows $C$-term intensity is inversely proportional to temperature in the linear limit $\left(\mathrm{k}_{\mathrm{B}} \mathrm{T}>g \beta H\right)$. Similar behavior occurs when there is a low-lying thermally accessible excited state that interacts with the ground state through an off-diagonal matrix element

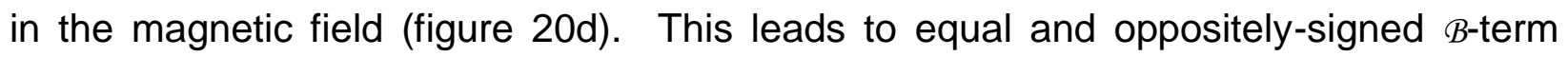
MCD intensity from the interacting levels. Since the ground state is preferentially Boltzmann-populated at low temperature, this leads to a temperature dependent $\mathscr{B}$-term. If the interacting levels are separated by an amount that is comparable to the Zeeman splitting $(\Delta \mathrm{E} \sim \mathrm{g} \beta \mathrm{H})$ this results in a non-linear temperature dependent $\mathfrak{B}$-term (see 3.3.3, figure 22).

The information content of $C$-term MCD intensity is high. ${ }^{122}$ It reflects magnetic properties of the ground state and excited state involved in the MCD transition, including ground state spin Hamiltonian parameters (see 3.3.3) and excited state $g$-values and spin-orbit coupling. This can be seen from the expression for orientation averaged $C^{-}$ term intensity for a transition between the ground state $A$ and excited state $\mathrm{J}$ :

$$
C_{0}=-\frac{1}{\left|3 d_{A}\right|} \sum\langle A|\hat{L}+2 \hat{S}| A\rangle \cdot\left(\left\langle A\left|\vec{\mu}_{e}\right| J\right\rangle \times\left\langle J\left|\vec{\mu}_{e}\right| A\right\rangle\right)
$$

In this expression, $d_{A}$ is the degeneracy of the ground state, $\mu_{\mathrm{e}}$ is the electric dipole operator, and the sum is carried out over all components of the ground state $A$ and excited state $J$. For the cross product in this expression to be non-zero, two non-zero perpendicular transition moments are required. This condition is satisfied if either the ground or excited state carries orbital angular momentum. On the other hand, 
transitions between orbitally non-degenerate states can only have a transition moment in a single direction. Paramagnetic species with orbitally non-degenerate ground states can exhibit significant $C$-term intensity, however. In this case, spin-orbit coupling mixes states with different polarizations, satisfying the requirement for two perpendicular transition moments. (Note that the spin-orbit mixing must occur along a third, mutually perpendicular direction.) The magnitude of spin-orbit coupling is high for transition metal centers relative to e.g. organic ligands. This, combined with the inherently strong spin-orbit mixing of ligand field states means that $d$ - $d$ transitions can be significantly enhanced in low-temperature MCD relative to Abs. The ability to resolve these otherwise weak features is a key advantage of MCD. Since the spin-orbit mixing of charge-transfer states is generally weaker, charge-transfer enhancement in MCD (relative to Abs) is not nearly as pronounced. It is therefore possible to discriminate between ligand field and charge transfer excitations based on their relative intensities in MCD and Abs. Finally from eqn 8, if the g-values (derived from $\langle L+2 S\rangle)$ are known (e.g. from EPR - see 3.4.1), it is possible to experimentally determine the polarization of an MCD transition even in a randomly oriented sample. If the orientation of the g tensor is known relative to the molecular axes, this can greatly facilitate spectral assignments especially for charge-transfer transitions, which tend to be polarized along M-L bonds. 
3.3.3 - Saturation Magnetization: Variable-Temperature Variable-Field MCD. For a paramagnetic transition metal site, $c$-term MCD intensity reflects the magnetization of the metal center. MCD can therefore provide the same ground state information as magnetic susceptibility, but with a number of distinct advantages. First, there is no diamagnetic contribution to $c$-term intensity. It is therefore straightforward to study the magnetization of $<1 \mathrm{wt} \%$ metal in diamagnetic matrix using MCD. This is particularly important for metal centers in metalloenyzmes and metallozeolites. Second, a component in a mixture can be studied selectively through its characteristic MCD features. This makes it possible to focus on the ground state of a single species, even if it exists within a distribution of paramagnetic spectator sites (as is often the case in metallozeolite chemistry). Finally, MCD offers additional information concerning the polarization of electronic transitions. ${ }^{19,113,122}$

Saturation magnetization occurs when the thermal energy is on the order of, or smaller than the Zeeman splitting of the ground state and the system is no longer in the Curie limit. Like magnetic susceptibility, $c$-term MCD intensity is studied using a saturation magnetization experiment conducted over a range of magnetic fields and low temperatures (typically 0-7 Tesla, 2-50 K). This is known as variable-temperature variable field MCD (VTVH-MCD). In the limit of low temperature or high magnetic field, there is a deviation from Curie Law behavior (intensity linear in $1 / T$ ), and at high magnetic fields and low temperature, $C$-term intensity becomes field- and temperatureindependent. This corresponds to the case where the Zeeman splitting $g \beta H$ is large relative to $\mathrm{k}_{\mathrm{B}} \mathrm{T}$, and only the lowest component of the ground state is Boltzmann populated. This approach to this limit is described by the Brillouin curves shown in 
figure 21 , which are sensitive to spin state and molecular $g$-values, but only depend on $H / T$. The saturation magnetization MCD of $\mathrm{S}>1 / 2$ metals is generally more complicated than the behavior shown in figure 21 ,



however. This is a consequence of ZFS Figure 21. Simulated MCD Brillouin curves for isotropic $S=1 / 2-5 / 2$ ground states. Adapted from reference ${ }^{122}$ with permission. Copyright 1999 American Chemical Society.

(see 3.1.1). For systems with axial ZFS $(D \neq 0)$, the Zeeman levels of the ground state will be separated in energy in the absence of a magnetic field. Since MCD intensity from a given Zeeman level is proportional to $\left\langle\mathrm{S}_{\perp}\right\rangle$, the spin projection of the level on the axis mutually perpendicular to the two non-zero

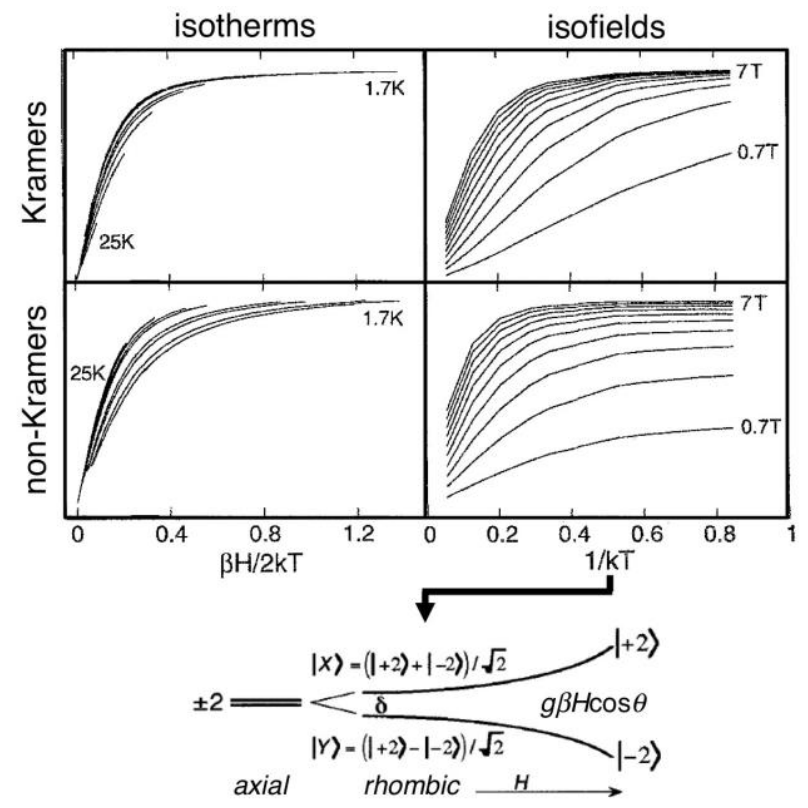

transition moments (e.g. $<\mathrm{S}_{z}>$ for an $x y$ polarized transition), differential Boltzmann population of Zeeman levels with different $\left\langle\mathrm{S}_{\perp}\right\rangle$ leads to nested saturation magnetization isotherms (i.e. non-superimposed curves in the $\mathrm{H} / \mathrm{T}$ plots shown in figure 22). The

Figure 22. VTVH-MCD saturation magnetization isotherms (left) and isofields (right) for a rhombic $S=2$ system, including the effects of rhombic ZFS and magnetic field $\mathrm{H}$ on $a \mathrm{M}_{\mathrm{s}}= \pm 2$ non-Kramers doublet (bottom right). Analagous VTVH-MCD data are presented for an $S=5 / 2$ Kramers system (middle). The $S=2$ and $S=5 / 2$ isotherms display nesting behavior (i.e. do not overlay) due to differential Boltmann population of zero-field split levels with different spin expectation values. The $S=2$ isofields show field dependence in the low temperature saturation limit, reflecting variation of the non-Kramers ground state with field. No such effect is seen in the $\mathrm{S}=5 / 2$ isofields, reflecting the lack of field dependence in the ground state of Kramers ions. 
magnitude of this effect reflects the magnitude of $D$. For non-Kramers ions, there are additional effects due rhombic ZFS $(E \neq 0)$. For these systems, the ground state, which is an equal mixture of $|+2\rangle$ and $|-2\rangle$ (see bottom of figure 22) will have a spin expectation value $\left\langle S_{\perp}>=0\right.$ resulting in no MCD intensity (see 3.1.1). In the presence of a magnetic field, however, the ground state becomes MCD active through a field-induced mixing of the zero-field split levels. ${ }^{126}$ As shown in the bottom right of figure 22, these become the pure $|+2\rangle$ and $|-2\rangle$ states at high magnetic fields. This results in a non-zero $\left\langle S_{\perp}\right\rangle$ when the Zeeman interaction becomes large relative to the rhombic splitting ( $\delta$ in figure 22, bottom right). Evidence for this is seen in the saturation magnetization isofields of integer-spin metals (figure 22, middle right), where variation of the ground state wavefunction with magnetic field leads to magnetic field dependence in the lowtemperature (large 1/T) saturation limit (figure 22, bottom right). This effect can be quantified by VTVH-MCD, and then related to the energetic separation $\delta$ between the field-mixed Zeeman levels (figure 22, bottom right). For both Kramers and non-Kramers systems, Spin Hamiltonian parameters (see 3.1.1) are extracted by fitting VTVH-MCD isotherms/isofields.

3.4 - Other Spectroscopic Methods With Single-Site Resolution. While siteselective spectroscopy provides the most reliable data on metallozeolite active sites, useful insight is also derived from techniques where features of single sites can be resolved from a mixture. In metallozeolite chemistry, electron paramagnetic resonance spectroscopy and Mössbauer spectroscopy have proven to be particularly useful. 
Nuclear resonance vibrational spectroscopy also holds significant potential for Fezeolites.

3.4.1 - Electron Paramagnetic Resonance Spectroscopy. Electron paramagnetic resonance spectroscopy (EPR) is used to probe transitions between Zeeman-split sublevels of a paramagnetic ground state. ${ }^{107,108,110,115}$ EPR can be used to measure $g$ values and ZFS parameters reflecting the electronic structure of the metal center (see 3.1.1) with high accuracy. The high energy resolution of EPR also enables detection of small magnetic couplings between unpaired electrons and metal/ligand nuclear spins that are inaccessible using saturation magnetization experiments (e.g. magnetic susceptibility, VTVH-MCD). These hyperfine and superhyperfine interactions provide a direct experimental handle on metal-ligand covalency (see 3.1.1). 
A
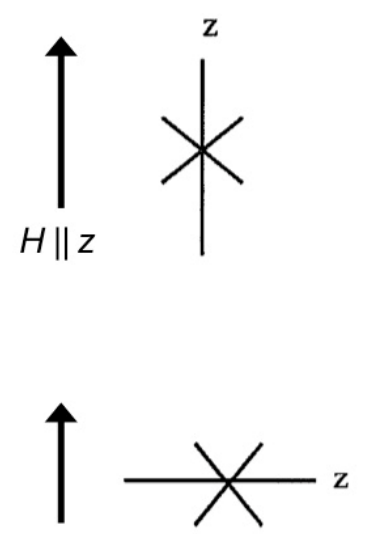

$H \perp z$
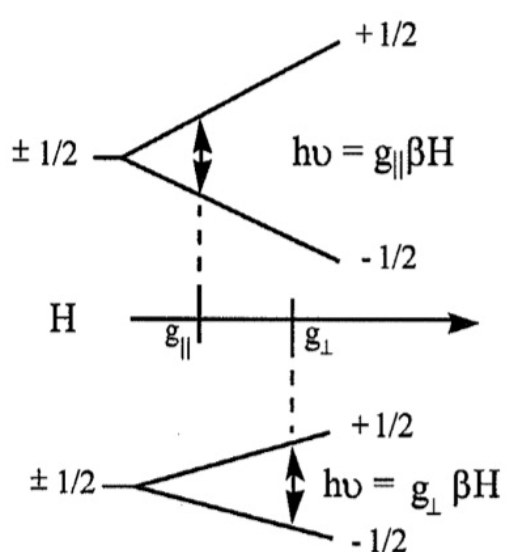

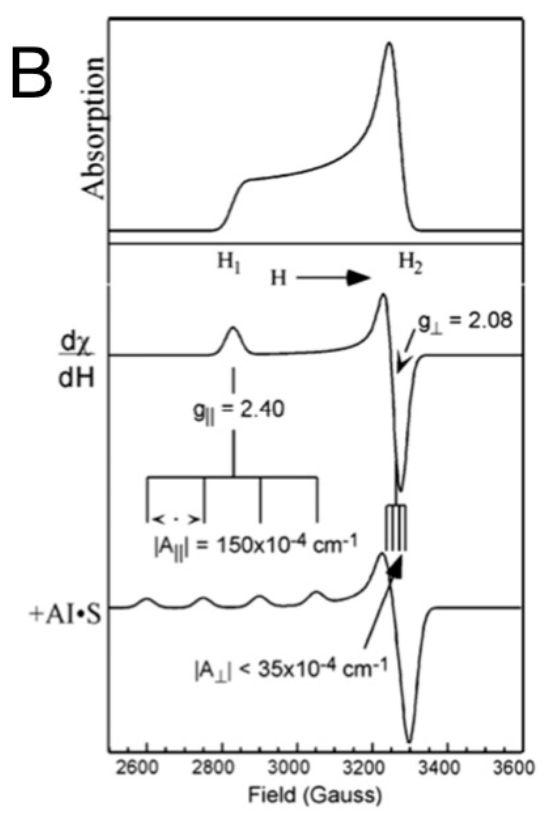

Figure 23. (A) Orientation-dependence of the Zeeman splitting of an $S=1 / 2$ ground state due to $g$ anisotropy. (B) Simulated EPR absorption spectrum of an axial $\mathrm{Cu}$ (II) center (top), its first derivative (middle), including effects from hyperfine coupling to the $\mathrm{I}=3 / 2$ nuclear spin of $\mathrm{Cu}$ (bottom).

In the standard EPR experiment, the sample is irradiated with a fixed microwave frequency $\left(\mathrm{X}\right.$ band, at $9 \mathrm{GHz} \approx 0.3 \mathrm{~cm}^{-1}$ ) in the presence of a magnetic field oriented perpendicular to the microwave $B$ field. The magnetic field is swept, and microwave radiation is absorbed when the Zeeman splitting between levels with $\Delta \mathrm{M}_{s}= \pm 1$ is brought into resonance with the microwave energy. As shown in figure $23 a$, this induces a magnetic dipole transition between Zeeman-split levels, and the resonance field associated with this transition is a function of the molecular $g$ values and the orientation of the molecule with respect to the magnetic field. For a frozen solution or powder, the EPR spectrum is a weighted sum of contributions from all orientations (contribution increasing from $\|$ to $\perp$, as shown in the top of figure 23b). Since each orientation is weighted differently, it is possible to measure anisotropic quantities (e.g. g-values and hyperfine couplings) from a randomly oriented sample. High sensitivity is achieved by modulating the external field and using lock-in detection, which leads to a $1^{\text {st }}$ derivative 
spectrum. For example, a spin $1 / 2$ system such as $\mathrm{Cu}(\mathrm{II})$ will have an absorption spectrum similar to $23 \mathrm{~b}$ (top), whose first derivative $\mathrm{d} \chi / \mathrm{dH}$ (23b, middle) shows distinct features at the resonance fields corresponding to $g_{\|}$and $g_{\perp} .{ }^{18}$ (These deviate from the free electron g-value of 2.0023 due to spin-orbit coupling with ligand field excited states - see 3.1.1.) Additional features arise due to hyperfine interactions between the unpaired electron spin and the nuclear spin of the metal (see 3.1.1). For $\mathrm{Cu}(\mathrm{II})$, the $\mathrm{S}=1 / 2$ ground state couples to the $\mathrm{I}=3 / 2$ nuclear spin of $\mathrm{Cu}$, splitting spectral features into $2 \mathrm{l}+1=4$ lines (figure $23 \mathrm{~b}$, bottom). The hyperfine splittings of the $g_{\|}$and $g_{\perp}$ resonances will in general be different, reflecting the anisotropy of the hyperfine interaction. In some cases, it is also possible to resolve small superhyperfine couplings arising from interactions between the unpaired electron spin and the nuclear spin of ligands (see 3.1.1). Hyperfine and superhyperfine coupling provide a direct probe of the covalency of the ligand-metal bond. For molecules with $S>1 / 2$, the ground state will experience zero-field splitting when the molecule has axial or lower symmetry. This is described by the phenomenological ZFS spin Hamiltonian discussed in 3.1.1, and characterized by the ZFS parameters $E$ and $D$ introduced in equation 5 . In the limit that the zero-field splitting is large relative to the Zeeman splitting $(|\mathrm{D}|>>\mathrm{g} \beta \mathrm{H}$ for magnetic 
field strengths used in X-band EPR), the zero-field split sublevels behave as isolated doublets with unusual effective g-values. For example, for an axial high spin $\mathrm{Fe}(\mathrm{III})$ site with molecular g-values $g_{\perp}=g_{\|}=2$, the $M_{s}= \pm 1 / 2, \pm 3 / 2, \pm 5 / 2$ Kramers doublets will have anisotropic effective $\mathrm{g}$ values ranging from 0-10 (figure 24a). This significantly impacts the resonance positions observed in EPR for high spin $\mathrm{Fe}$ (III) relative to a simple $\mathrm{S}=1 / 2$ system
$A$

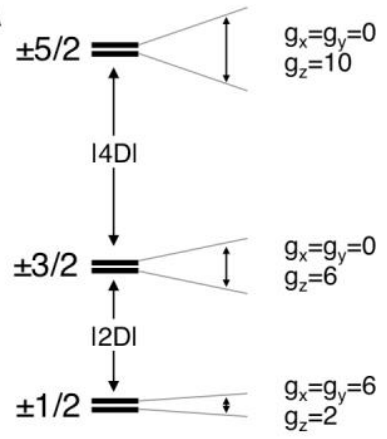

$\mathrm{D}>\mathrm{OH} \longrightarrow$

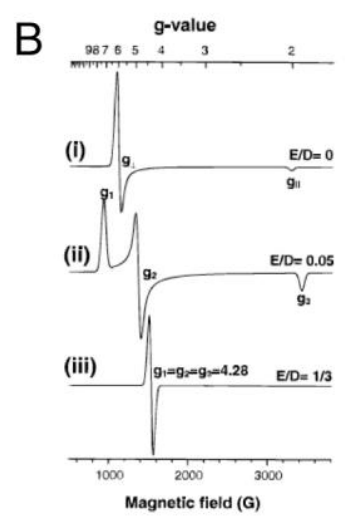

Figure 24. (A) Orientation-dependent response of an axial zero-field split $S=5 / 2$ spin to magnetic field applied along the $x, y, z$ directions. (B) Simulated EPR spectra from axial (i, $E / D=0$ ), rhombic perturbed (ii, $E / D=0.06$ ), and maximally rhombic (iii, $E / D=1 / 3 S=5 / 2$ ground states. The Rhombicity parameter $E / D$ is defined based on the zero-field splitting parameters $E$ and $D$ in equation 5 . E/D deviates from zero for geometries in which the $x$ and $y$ directions are inequivalent.

(figure 24b). The resonance positions of high spin Fe(III) are also highly sensitive to the presence of rhombic ZFS (figure 24b), and EPR enables direct quantification of rhombicity (E/D), and temperature-dependent studies can be used to assign the sign and magnitude of $D$.

In contrast to $\mathrm{Fe}(\mathrm{III})$, integer spin metals such as high spin $\mathrm{Fe}(\mathrm{II})$ are generally inaccessible using the standard X-band EPR experiment. This is because the zero-field splitting between levels with $\Delta M_{s}= \pm 1$ is generally too large to be brought into resonance with the $\mathrm{X}$-band microwave energy $\left(0.3 \mathrm{~cm}^{-1}\right)$. For negative zerofield split non-Kramers ground states, it is in some cases possible to probe the transition between

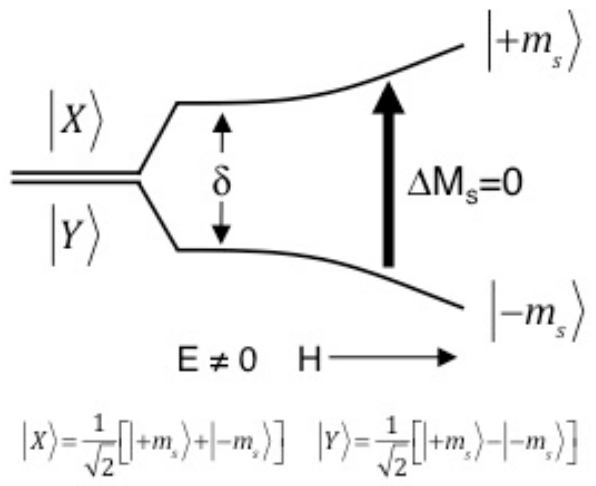

Figure 25. Non-Kramers doublet with rhombic splitting $\delta$ smaller than the microwave frequency, enabling detection of the $\Delta M_{s}=0$ transition. 
components of the lowest non-Kramers doublet due to the rhombic mixing of the spin sublevels (figure 25). ${ }^{127,128}$ This is done using parallel-mode EPR, where the B field of the microwave is oriented parallel to the external magnetic field resulting in the selection rule $\Delta \mathrm{M}_{\mathrm{s}}=0$. Note that, while non-Kramers ions are generally difficult to study using EPR, they are amenable to saturation magnetization experiments such as VTVH-MCD (see 3.3.3). ${ }^{19,116}$

3.4.2 - Mössbauer Spectroscopy. Mössbauer spectroscopy is an element-specific technique that probes the resonant absorption of $\gamma$ photons by Mössbauer-active nuclei. ${ }^{119}$ It is most commonly used to study the chemical environment of the ${ }^{57} \mathrm{Fe}$ nucleus, which is sensitive to oxidation state, spin state, and coordination geometry (vide infra). This experiment uses a ${ }^{57}$ Co source, which decays through electron capture to form a metastable nuclear excited state of ${ }^{57} \mathrm{Fe}$. The excited ${ }^{57} \mathrm{Fe}$ nucleus subsequently relaxes to its ground state, emitting a $14.4 \mathrm{keV}$ gamma ray. This $\gamma$ almost exactly spans the gap between the $I=1 / 2$ ground state and metastable $I=3 / 2$ nuclear excited state of the ${ }^{57} \mathrm{Fe}$ nucleus $\left({ }^{57 \mathrm{~m}} \mathrm{Fe}\right.$, figure $\left.26 \mathrm{a}\right)$. However it will not be in exact resonance due to the differing chemical environments of the source and the absorber. The $\gamma$ can be brought into resonance using the Doppler effect, accomplished by varying the velocity of the ${ }^{57} \mathrm{Co}$ source toward or away from the sample (usually over a range of $\pm 10 \mathrm{~mm} / \mathrm{s}$, corresponding to Doppler shifts ranging over $\left.\pm 3.87 \times 10^{-3} \mathrm{~cm}^{-1}\right)$.

As shown in figure 26b, the Mössbauer spectrum of an isolated (mononuclear) iron center will generally appear as a doublet of Lorentzian lines. (Additional features arise for slowly-relaxing paramagnetic sites, or with the application of an external 

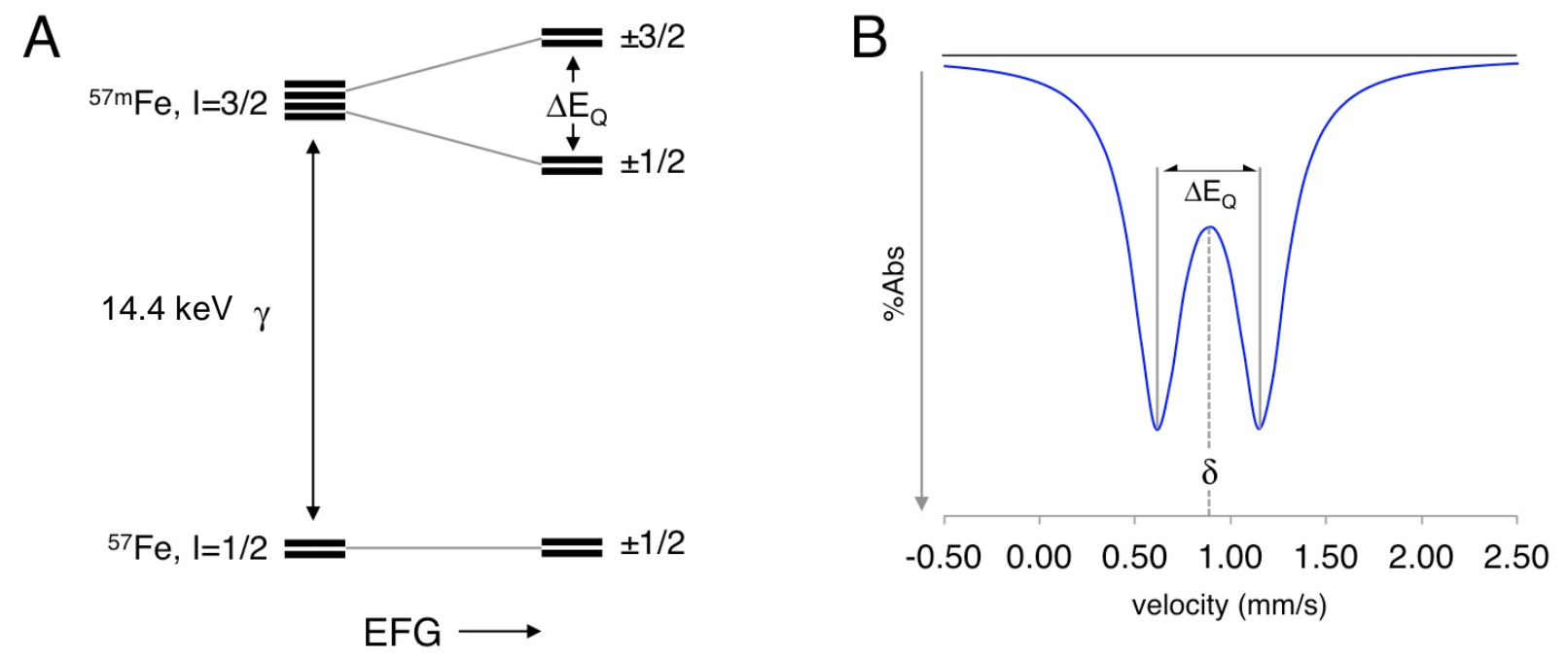

Figure 26. (A) The Mössbauer transition, including effects due to a non-zero electric field gradient (EFG) at the ${ }^{57} \mathrm{Fe}$ nucleus $\left(\Delta \mathrm{E}_{\mathrm{Q}}>0\right)$. (B) Simulated Mössbauer spectral data, with the isomer shift $(\delta)$ and quadrupole splitting $\left(\Delta \mathrm{E}_{\mathrm{Q}}\right)$ indicated.

magnetic field.) The center point of the doublet, $\delta$, is known as the isomer shift. As shown in equation $9, \delta$ reflects the difference in electron density at the nucleus of the absorber $\left|\Psi_{0}\right|^{2} \mathrm{~A}$ versus the source $\left|\Psi_{0}\right|^{2} \mathrm{~s}$, as well as the relative change in nuclear radius upon nuclear excitation $(\partial \mathrm{R} / \mathrm{R})$ :

$$
\delta=(\text { constant })(\partial \mathrm{R} / \mathrm{R})\left(\left|\Psi_{0}\right|^{2} \mathrm{~A}-\left|\Psi_{0}\right|^{2} \mathrm{~S}\right)
$$

For iron, $\partial \mathrm{R} / \mathrm{R}$ is negative. A positive $\delta$ therefore reflects reduced electron density at the nucleus. Since $\left|\Psi_{0}\right|^{2}$ derives mostly from electrons in core $s$ orbitals, electrons in valence $3 d$ orbitals affect $\delta$ indirectly, decreasing $\left|\Psi_{0}\right|^{2}$ through shielding effects (mostly from $3 s$ ). $\delta$ is therefore sensitive to oxidation state and spin state. The Mössbauer transition is generally split into two lines, reflecting the interaction between the electric quadrupole moment of the ${ }^{57 m} \mathrm{Fe}$ nuclear excited state with the electric field gradient 
(EFG) at the nucleus. This interaction splits the $I=3 / 2$ excited state into $M_{l}= \pm 1 / 2, \pm 3 / 2$ doublets separated by the amount $\Delta \mathrm{E}_{\mathrm{Q}}$ (figure 26). The interaction energy, called the quadrupole splitting, will be non-zero for sites with non-cubic distributions of electron density:

$$
\Delta E_{Q}=\frac{e Q}{2} V_{Z Z} \sqrt{\left(1+\frac{\eta^{2}}{3}\right)}
$$

In this expression, $e$ is the electron charge, $Q$ is the nuclear quadrupole moment (positive for $\left.{ }^{57 \mathrm{~m} F e}\right), \quad \eta=\left(V_{x x}-V_{y y}\right) / V_{z z}$ is the asymmetry parameter, and the $V_{i i}$ are eigenvalues of the EFG tensor. For sites with cubic molecular symmetry, differential population of the valence $3 d$ orbitals leads to non-cubic electron density, and the $V_{i i}$ will become non-zero. This 'valence' contribution to $\Delta \mathrm{E}_{\mathrm{Q}}$ is highly sensitive to oxidation state and spin state, as these affect $d$ orbital occupancy. Deviations from cubic site symmetry lead to an additional 'lattice' contribution to $\Delta \mathrm{E}_{\mathrm{Q}}$ arising from a non-cubic distribution of ligand electron density. (While $\Delta \mathrm{E}_{\mathrm{Q}}$ can be separated into valence and lattice contributions for ionic complexes, this distinction is less meaningful for highly covalent active sites.)

\subsection{3 - Nuclear Resonance Vibrational Spectroscopy. Whereas Mössbauer} spectroscopy is used to probe resonant absorption by the ${ }^{57} \mathrm{Fe}$ nucleus, nuclear resonance vibrational spectroscopy (NRVS) is a synchrotron-based technique used to study vibrational side-bands of the Mössbauer transition. ${ }^{129,130}$ Since NRVS involves inelastic absorption, conservation of momentum dictates the excess linear momentum 
of the photon be transferred to the absorbing ${ }^{57} \mathrm{Fe}$ nucleus. As a result, vibrations that contain iron motion are active in NRVS, and NRVS intensity correlates to the magnitude of $\mathrm{Fe}$ displacement in a particular normal mode at a given energy. NRVS is therefore sensitive to low-energy (150$600 \mathrm{~cm}^{-1}$ ) core vibrations of $\mathrm{Fe}$ active sites. ${ }^{131-133}$ These vibrations are highly

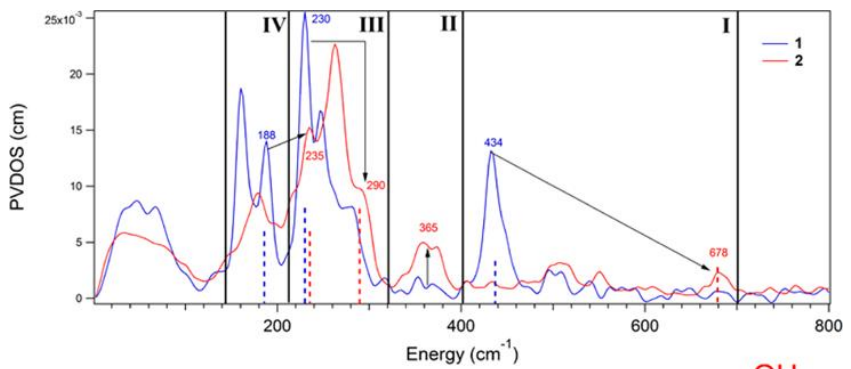
sensitive to coordination geometry, and
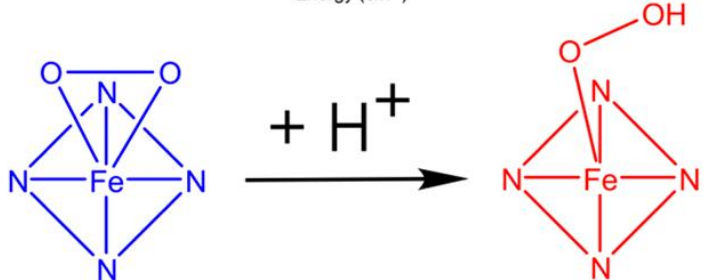

Figure 27. NRVS spectral changes associated with the protonation of the side-on peroxoferric complex [(TAML)Fe $\left.{ }^{\text {III }}(\mathrm{OO})\right]^{+}$(blue) to form the corresponding end-on hydroperoxide complex (red). Reproduced with permission from reference ${ }^{240}$. Copyright 2016 American Chemical Society.

can be difficult to resolve using other vibrational techniques such as resonance Raman spectroscopy (sensitive to higher energy vibrations reflecting excited state distortions). NRVS has therefore been used to evaluate structures for intermediates across many mono- and binuclear Fe enzymes and model complexes (see figure 27 for example). These studies are now being extended to iron active sites in zeolites, and this will provide detailed structural information that is currently inaccessible using other techniques. 
$4-\mathrm{Cu} / \mathrm{O}$ Active Sites in Zeolites. Cu-zeolites carry out diverse functions ranging from reduction of $\mathrm{NO}_{x}$ pollutants to selective hydrocarbon oxidation using atmospheric $\mathrm{O}_{2}$. The best-studied system, Cu-ZSM-5, is notable for hydroxylating methane rapidly at low temperatures $\left(150{ }^{\circ} \mathrm{C}\right)$ to form methanol. ${ }^{36,37}$ Reactivity occurs at a binuclear extralattice $\mathrm{Cu}$ active site, which is activated by either $\mathrm{O}_{2}$ or $\mathrm{N}_{2} \mathrm{O}$ to form the reactive intermediate in methanol synthesis. An experimental approach based on site-selective spectroscopy was developed to distinguish this minority species from 95\% spectator $\mathrm{Cu}$, and coupled to DFT, this defined the geometric and electronic structure of the active site and elucidated its reactivity toward strong $\mathrm{C}-\mathrm{H}$ bonds. ${ }^{37}$ These studies were extended to analogous active sites in other lattice topologies - notably Cu-MOR - as well as the precursor intermediates formed during the activation of $\mathrm{O}_{2} / \mathrm{N}_{2} \mathrm{O}$. ${ }^{66,134-136}$ Different catalyst preparations are also believed to result in methane-reactive $\mathrm{Cu}$ sites with different nuclearities (e.g. trinuclear, mononuclear). ${ }^{55,74}$ However these possibilities have not yet been evaluated using site selective spectroscopy.

\section{1 - Methane Hydroxylation by Cu-ZSM-5. Activating Cu-ZSM-5 under an} atmosphere of either $\mathrm{O}_{2}$ at $450^{\circ} \mathrm{C}$ or $\mathrm{N}_{2} \mathrm{O}$ at $150-200{ }^{\circ} \mathrm{C}$ leads to the formation of an intense absorption feature centered at $22,700 \mathrm{~cm}^{-1}$ (figure 28A) that decays in the presence of methane (figure 28B). ${ }^{36,37}$ This absorption feature is correlated with the production of methanol, and is therefore a spectroscopic handle of the Cu active site of methanol synthesis. ${ }^{37}$ As shown in figure $28 \mathrm{C}$, the decay of the absorption feature can be used to track the kinetics of methane hydroxylation. This provided an apparent 
activation energy of $15.7 \mathrm{kcal} / \mathrm{mol}$, and an H/D kinetic isotope effect that is consistent with $\mathrm{C}-\mathrm{H}$ cleavage in the rate limiting step (see section 4.2 for detail).

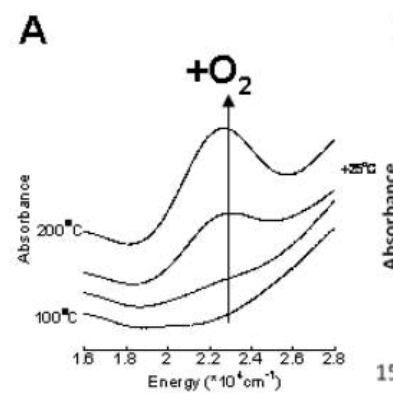

B

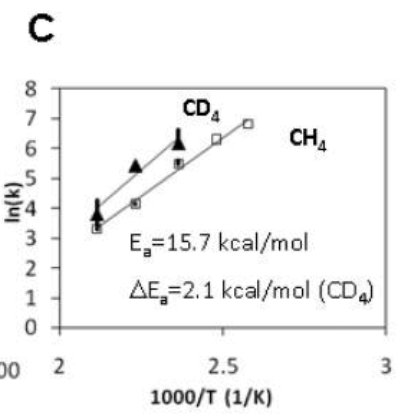

Figure 28. A) Growth of the $22,700 \mathrm{~cm}^{-1}$ absorption feature of the active site during $\mathrm{O} 2$ activation and $\mathrm{B}$ ) its subsequent decay upon interaction with methane. C) kinetics of methane hydroxylation from decay of the $22,700 \mathrm{~cm}^{-1}$ band. Time interval between spectra is 15 seconds. Adapted from reference ${ }^{212}$ with permission from the Royal Society of Chemistry.

The $22,700 \mathrm{~cm}^{-1}$ Abs feature was used to selectively probe this active site that exists in a dominant (95\%) distribution of spectator $\mathrm{Cu} .{ }^{37}$ Tuning a laser into the 22,700 $\mathrm{cm}^{-1}$ band enabled its study by resonance Raman spectroscopy (figure 29, inset $A$ ). This selectively resonance enhances vibrations of the active site, shown in figure 29. Work from the Solomon group has defined the characteristic resonance Raman vibrations of all known structures in $\mathrm{Cu} / \mathrm{O}$ chemistry (table 5), and these were compared to data from activated Cu-ZSM-5. The active site was originally thought to be a bis- $\mu-$ oxo $2 \mathrm{Cu}(\mathrm{III})$ species based on the energy of its $22,700 \mathrm{~cm}^{-1}$ absorption feature, ${ }^{36}$ however this was excluded by $r R$, as there is is no intense ${ }^{16 / 18} \mathrm{O}$-sensitive vibration at $600 \mathrm{~cm}^{-1}$ in figure 29 (characteristic of a bis- $\mu$-oxo core - table 5 ). ${ }^{137,138} \mathrm{Cu}$ (II)superoxide structures were also excluded due to the absence of an isotope sensitive vibration at $\sim 1100 \mathrm{~cm}^{-1}$ (table 5). ${ }^{139,140}$ On the other hand, the vibration at $870 \mathrm{~cm}^{-1}$ with a ${ }^{16 / 18} \mathrm{O}$ isotope shift of $40 \mathrm{~cm}^{-1}$ in figure 29 is in the correct range for a $\mathrm{Cu} / \mathrm{OOH}$ species. ${ }^{141}$ To evaluate this possibility, mixed-isotope ${ }^{16 / 18} \mathrm{O}_{2}$ labeling studies were performed to test for the presence of an O-O bond. Cu-ZSM- 5 was activated with a 
statistical $\quad(1: 2: 1) \quad$ mixture of

${ }^{16} \mathrm{O}_{2} /{ }^{16 / 18} \mathrm{O}_{2} /{ }^{18} \mathrm{O}_{2}$. If an $\mathrm{O}-\mathrm{O}$ bond were

present, vibrations would be observed at

$870 \mathrm{~cm}^{-1}, \sim 845 \mathrm{~cm}^{-1}$, and $830 \mathrm{~cm}^{-1}$ with a

1:2:1 intensity distribution. As shown in

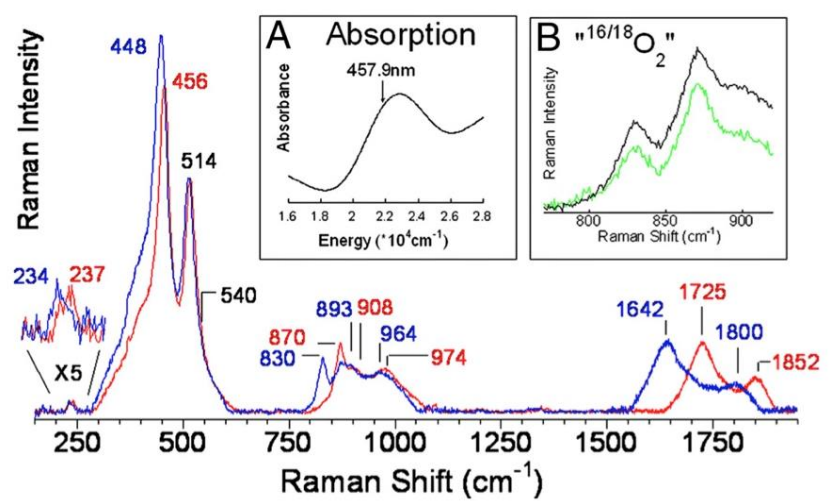

figure 29 inset B (green spectrum), no

intermediate frequency mode is

Figure 29. Resonance Raman spectra $\left(\lambda_{\text {ex }}=458 \mathrm{~nm}\right.$ - see inset A) of Cu-ZSM-5 activated with ${ }^{16} \mathrm{O}_{2}$ (red) and ${ }^{18} \mathrm{O}_{2}$ (blue). Inset (B) shows a comparison of spectra in the $800-900 \mathrm{~cm}^{-1}$ region following activation with a 1:2:1 statistical mixture of ${ }^{16} \mathrm{O}_{2}:{ }^{16 / 18} \mathrm{O}_{2}:{ }^{18} \mathrm{O}_{2}$ (green) with summed spectra following ${ }^{16} \mathrm{O}_{2}$ and ${ }^{18} \mathrm{O}_{2}$ activation (black). Reproduced from reference ${ }^{37}$. present in the reactive intermediate. ${ }^{37}$ All Copyright 2009 United States National Academy of Science.

known structures (table 5) were therefore excluded for Cu-ZSM-5, indicating the active site was a new species in $\mathrm{Cu} / \mathrm{O}$ chemistry.

In the rR spectrum of activated Cu-ZSM-5, the most intense isotope sensitive vibration is observed at $456 \mathrm{~cm}^{-1}\left(\Delta^{16 / 18} \mathrm{O}=8 \mathrm{~cm}^{-1}\right)$. There is also a weak vibration at 870 $\mathrm{cm}^{-1}$ that exhibits a large ${ }^{16 / 18} \mathrm{O}$ isotope shift $\left(40 \mathrm{~cm}^{-1}\right)$. Based on frequency and

Table 5. Characteristic resonance Raman vibrations and their $16 / 18 \mathrm{O}$ isotope shifts for known $\mathrm{Cu} / \mathrm{O}$ intermediates. Reproduced with permission from reference ${ }^{37}$. Copyright 2009 United States National Academy of Sciences

isotope shift, the second quantum of this

vibration is seen at $1725 \mathrm{~cm}^{-1}$ $\left(\Delta^{16 / 18} \mathrm{O}=83 \mathrm{~cm}^{-1}\right)$, and it is six times as intense as the fundamental. The same intensity distribution is seen in mono- $\mu$ oxo bridged diferric sites. ${ }^{142}$ This led to the assignment of the active site as a

\begin{tabular}{|c|c|}
\hline $\mathrm{Cu} / \mathrm{O}_{2}$ species & $\begin{array}{l}\text { rR vibrations } \\
\left(\Delta^{18} \mathrm{O}_{2}\right) / \mathrm{cm}^{-1}\end{array}$ \\
\hline $\mathrm{O}_{2}$ activated $\mathrm{Cu}-\mathrm{ZSM}-5$ & $\begin{array}{c}456(8) \\
870(40) \\
\end{array}$ \\
\hline bis( $\mu$-oxo) dicopper(III) & $v_{\mathrm{Cu}-\mathrm{O}}=606(23)$ \\
\hline$\mu-\left(\eta^{2}: \eta^{2}\right)$ peroxo dicopper(II) & $\begin{array}{l}v_{\mathrm{C}_{1-C u}}=284(0) \\
v_{0-0}=763(40)\end{array}$ \\
\hline$\eta^{1}$-superoxo copper(II) & $\begin{array}{l}v_{C u-0}=472(20) \\
v_{0-0}=1121(63)\end{array}$ \\
\hline$\eta^{2}$-superoxo copper(II) & $\begin{array}{l}v_{\mathrm{C}-0-0}=554(20) \\
v_{0-0}=1043(59)\end{array}$ \\
\hline$\eta^{1}$-hydroperoxo copper(II) & $\begin{array}{l}v_{\mathrm{Cu}-\mathrm{O}}=624(17) \\
v_{\mathrm{O}-\mathrm{O}}=843(44)\end{array}$ \\
\hline trans- $\mu-1,2$-peroxo dicopper(II) & $\begin{array}{l}v_{\mathrm{Cu}-\mathrm{O}}=561(26) \\
v_{\mathrm{O}-\mathrm{O}}=832(44)\end{array}$ \\
\hline$\mu-1,1$-hydroperoxo dicopper(II) & $\begin{array}{l}v_{\text {Cu-O }}=322(10) \\
v_{0-0}=892(52)\end{array}$ \\
\hline
\end{tabular}


mono- $\mu$-oxo bridged dicopper site. The intense $456 \mathrm{~cm}^{-1}$ feature therefore corresponds to the symmetric stretch of the $\mathrm{Cu}_{2} \mathrm{O}$ core, while the $870 \mathrm{~cm}^{-1}$ feature corresponds the antisymmetric stretch, which is forbidden in resonance Raman and therefore weak. At higher frequency, the $1725 \mathrm{~cm}^{-1}$ feature is the second quantum of the antisymmetric stretch, which is symmetric and therefore $r R$ allowed and intense relative to the fundamental. Finally, based on frequency and isotope shifts, the $1852 \mathrm{~cm}^{-1}\left(\Delta^{16 / 18} \mathrm{O}=52\right.$ $\mathrm{cm}^{-1}$ ) feature is assigned as the $4^{\text {th }}$ quantum of the symmetric stretch. This gains intensity through Fermi resonance with the 2 vas feature.

The symmetric and antisymmetric modes of the $\mathrm{Cu}_{2} \mathrm{O}$ core are particularly important as they are sensitive to the bridge angle of the three-atom core. A normal coordinate analysis of these modes and their isotope shifts provided an estimated

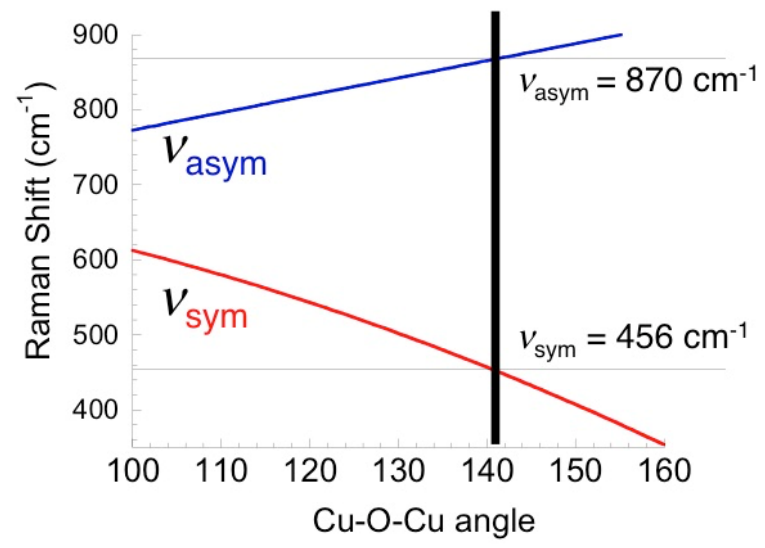
bridge angle of $140^{\circ}$ (figure 30). ${ }^{37}$ There is Figure 30. Predicted $v_{\text {sym }}$ and vasym frequencies as a function of $<\mathrm{CuOCu}$.

only one location in the ZSM-5 lattice where: 1) $\mathrm{Cu}$ is known to bind ${ }^{143}$ and 2) a methane-accessible $\mathrm{Cu}_{2} \mathrm{O}$ site with a $140^{\circ}$ bridge angle can be accommodated. This is the 10-membered ring motif shown in figure 31A. Within this ring, there are two aluminum T-sites separated by two silicon T-sites (i.e. an Al-O-Si-O-Si-O-Al sequence) that mediate metal binding. DFT indicates a $\mathrm{Cu}(\mathrm{III})-\mathrm{O}-\mathrm{Cu}(\mathrm{III})$ site is electronically unstable at this site, oxidizing the lattice ${ }^{37}$ On the other hand, this site does stabilize a $\mathrm{Cu}(\mathrm{II})-\mathrm{O}-\mathrm{Cu}(\mathrm{II})$ core with a $139^{\circ}$ bridge angle (figure $31 \mathrm{~B}$; versus $140^{\circ}$ from experiment) which reproduces the experimental Abs and resonance Raman spectroscopic features 
of the active site (figure $31 \mathrm{C}$ ). In this structure each $\mathrm{Cu}$ is three coordinate, bound in a bidentate fashion by two lattice $\mathrm{O}$ atoms derived from one $\mathrm{Al} \mathrm{T}$-site, as well as the bridging oxo ligand. The lattice modes seen in the experimental $r R$ spectrum at $514 / 540 \mathrm{~cm}^{-1}$ were assigned to the symmetric stretching mode of the bidentate Al T-site ligands. This site is highly covalent, with two electron holes delocalized over the $\left[\mathrm{Cu}_{2} \mathrm{O}\right]^{2+}$ core. TD-DFT predicts an intense oxo $\rightarrow \mathrm{Cu}(\mathrm{II})$ charge transfer at $23,400 \mathrm{~cm}^{-1}$ for the triplet $\left[\mathrm{Cu}_{2} \mathrm{O}\right]^{2+}$ model - in good agreement with the intense $22,700 \mathrm{~cm}^{-1}$ band in Abs spectroscopy. (The singlet and triplet models have nearly identical geometries, energies within $0.5 \mathrm{kcal} / \mathrm{mol}$, and highly similar predicted spectral features). The low energy and high intensity of this charge transfer band reflects the high covalency of the



10-membered ring
B
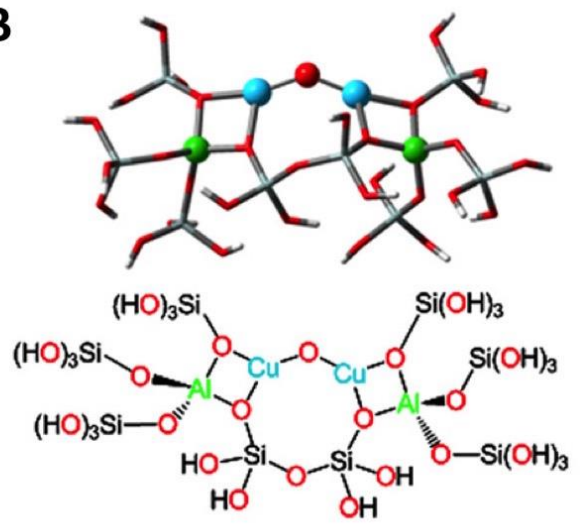

\begin{tabular}{|c|c|c|c|c|c|c|c|}
\hline \multirow{2}{*}{ C } & \multirow{2}{*}{$\begin{array}{c}\text { Abs features } \\
\text { LMCT }\end{array}$} & \multicolumn{3}{|c|}{ rR features } & \multicolumn{3}{|c|}{ Geometric parameters } \\
\hline & & $\begin{array}{c}v_{\text {sym }} \\
\left(\Delta^{18} \mathrm{O}_{2}\right) / \mathrm{cm}^{-1}\end{array}$ & $\begin{array}{c}v_{\text {asym }} \\
\left(\Delta^{18} \mathrm{O}_{2}\right) / \mathrm{cm}^{-1}\end{array}$ & $\begin{array}{c}v_{\text {lattice }} \\
\left(\Delta^{18} \mathrm{O}_{2}\right) / \mathrm{cm}^{-1}\end{array}$ & $<\mathrm{Cu}-\mathrm{O}-\mathrm{Cu}$ & Cu-O $(\AA)$ & Cu-Cu $(\AA)$ \\
\hline experiment & 22,700 & $456(-8)$ & $870(-40)$ & $514 / 540(0)$ & $140^{\circ}$ & - & - \\
\hline $\begin{array}{r}{\left[\mathrm{Cu}_{2} \mathrm{O}\right]^{2+}} \\
\text { model }\end{array}$ & 23,400 & $456(-5)$ & $852(-37)$ & $556 / 568(0)$ & $139^{\circ}$ & $1.75 / 1.76$ & 3.29 \\
\hline
\end{tabular}

Figure 31. A) 10-membered ring motif in the ZSM-5 lattice, with the Al-O-Si-O-Si-O-Al sequence implicated in $\mathrm{Cu}$ binding highlighted and $\mathrm{B}$ ) the $\left[\mathrm{Cu}_{2} \mathrm{O}\right]^{2+}$ cluster model derived from this motif. This model was generated by excising the cluster from the lattice in $A$, and capping its peripheral oxygen atoms with protons. C) Comparison of experimental and DFT-predicted $\left[\mathrm{Cu}_{2} \mathrm{O}\right]^{2+}$ spectral features and geometric structure. 
$\left[\mathrm{Cu}_{2} \mathrm{O}\right]^{2+}$ core, which is important for reactivity (see section 4.2, 6.3.1). ${ }^{37}$

4.2 - Reactivity of $\left[\mathrm{Cu}_{2} \mathrm{O}\right]^{2+}$ Active Sites in Zeolites. The kinetics of methane hydroxylation were evaluated by monitoring the disappearance of the $22,700 \mathrm{~cm}^{-1}$ Abs feature in the presence of methane (figure 28B,C). ${ }^{37}$ The reaction was performed at temperatures ranging from $110-200^{\circ} \mathrm{C}$, and the resulting Arrhenius plot gave an apparent activation barrier of $15.7 \mathrm{kcal} / \mathrm{mol}$. Performing the same experiment with $\mathrm{CD}_{4}$ resulted in a $3.1 \mathrm{kcal} / \mathrm{mol}$ higher barrier, leading to a large $\mathrm{H} / \mathrm{D}$ kinetic isotope effect (KIE) of 3.1 at $175^{\circ} \mathrm{C}$ - consistent with NMR product analysis of the reaction performed with mixed-isotope substrate $\left(\mathrm{CH}_{2} \mathrm{D}_{2}\right)$.

The observation of a large H/D KIE indicates $\mathrm{C}-\mathrm{H}$ cleavage occurs during the rate-limiting step, and this includes a small activation barrier for the strong, $104 \mathrm{kcal} / \mathrm{mol}$ $\mathrm{C}-\mathrm{H}$ bond of methane. The spectroscopically validated model of the Cu-ZSM-5 active site (vide supra) was used to computationally evaluate the ability of a $\left[\mathrm{Cu}_{2} \mathrm{O}\right]^{2+}$ core to cleave the $\mathrm{C}-\mathrm{H}$ bond of methane. On the triplet surface, the $\left[\mathrm{Cu}_{2} \mathrm{O}\right]^{2+}$ model reacts with

methane through $\mathrm{H}$-atom abstraction with a predicted activation barrier of $18.5 \mathrm{kcal} / \mathrm{mol}$ (versus $15.7 \mathrm{kcal} / \mathrm{mol}$ experimentally - see

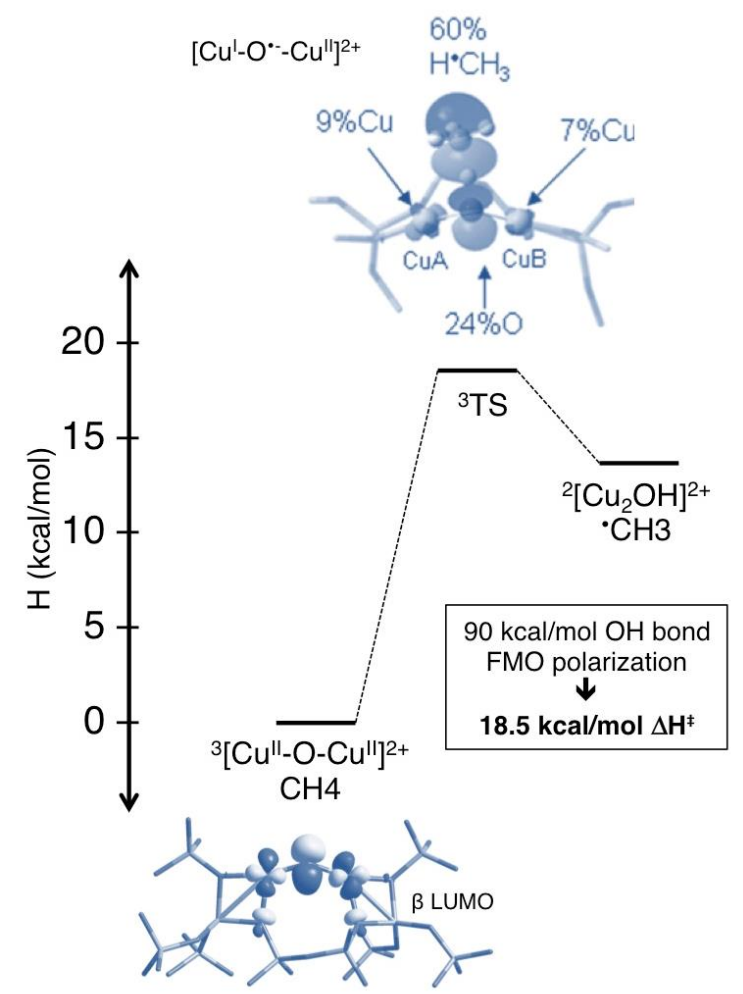

Figure 32. Methane $\mathrm{H}$-atom abstraction reaction coordinate on the triplet surface, highlighting changes in electronic structure that occur at the transition state geometry - in particular, polarization toward $[\mathrm{Cu}(\mathrm{I})-\mathrm{O} \cdot-\mathrm{Cu}(\mathrm{II})]^{2+}$. 
figure 32). ${ }^{37}$ This produces a mixed-valent $\mathrm{S}=1 / 2\left[\mathrm{Cu}_{2} \mathrm{OH}\right]^{2+}$ site coupled to a methyl radical, and the reaction is endothermic by just $13.8 \mathrm{kcal} / \mathrm{mol}$. The $13.8 \mathrm{kcal} / \mathrm{mol}$ reaction enthalpy reflects the difference in the strengths of the $\mathrm{C}-\mathrm{H}$ bond of methane (104 kcal/mol) and the $\mathrm{O}-\mathrm{H}$ bond of the hydroxo-bridged product. The driving force for forming a strong $90 \mathrm{kcal} / \mathrm{mol} \mathrm{O}-\mathrm{H}$ bond lowers the barrier for $\mathrm{H}$-atom abstraction. The electronic structure of the $\left[\mathrm{Cu}_{2} \mathrm{O}\right]^{2+}$ core was also found to be intrinsically activated toward $\mathrm{H}$-atom abstraction. In the triplet ground state, the electron holes from each $\mathrm{Cu}$ are significantly covalently delocalized onto the bridging oxo. These low-lying unoccupied orbitals therefore have high oxo $2 p$ content, and are good acceptor orbitals for $\mathrm{H}$-atom abstraction. As shown in the top of figure 32, methane interacts with the SOMO with oxo $2 p$ character oriented out of the Cu-O-Cu plane. At the transition state geometry, this hole localizes onto the bridging oxo, resulting in a $\mathrm{Cu}(\mathrm{I})$-oxyl-like electronic structure that is highly activated for HAA. ${ }^{37}$

Other Cu-zeolites are known to react with methane at low temperature, ${ }^{36,66,134}$ including $\mathrm{Cu}$ MOR, where methane reactivity is also correlated with an absorption feature at $\sim 22,000 \mathrm{~cm}^{-1} .36,134$ Unlike Cu-ZSM-5, the absorption maximum for $\mathrm{Cu}$ MOR shifts during reaction with methane. ${ }^{134}$ This is also seen in spectral changes accompanying desorption of the Cu-MOR active sites at high temperature. In figure 33 , heating $\mathrm{Cu}-\mathrm{MOR}$ to 450 ${ }^{\circ} \mathrm{C}$ in an atmosphere of $\mathrm{O}_{2}$ results in loss of an Abs

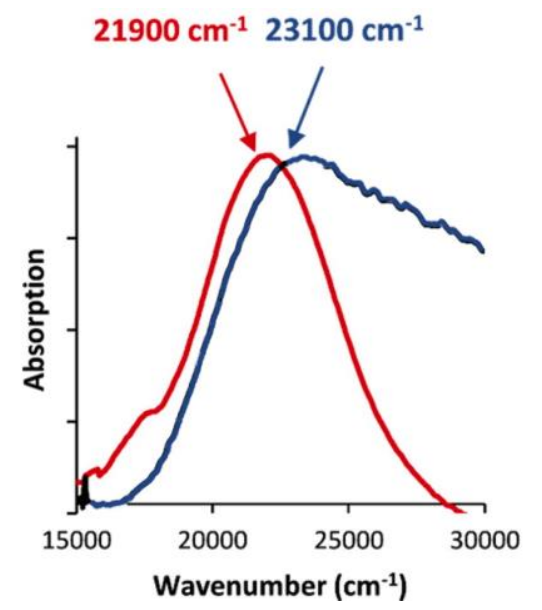

Figure 33. DR-UV-vis difference spectra of activated Cu-MOR showing components lost during heating in $\mathrm{O}_{2}$ atmosphere at $450^{\circ} \mathrm{C}$ (red trace), and

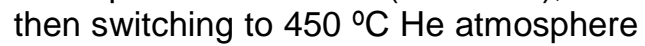
(blue trace). Adapted from reference 134 with permission. Copyright 2015, American Chemical Society. 
feature centered at $21,900 \mathrm{~cm}^{-1}$ (red trace).

The remaining Abs component centered at 23,100 $\mathrm{cm}^{-1}$ (blue trace) decays upon switching from $\mathrm{O}_{2}$ to $\mathrm{He}$ atmosphere (still at $\left.450{ }^{\circ} \mathrm{C}\right)$. This indicates two $\mathrm{Cu} / \mathrm{O}$ components contribute to the $22,000 \mathrm{~cm}^{-1}$ Abs feature, and kinetics studies show these

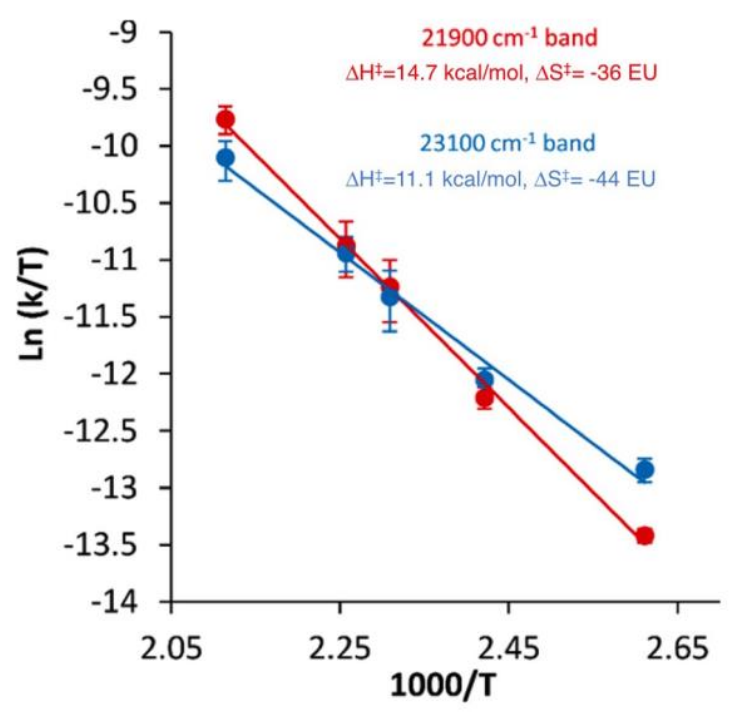

components react with methane at different rates. Two rates are required to fit the Arrhenius plot of the decay of this absorption

Figure 34. Eyring plot for the decay of the $21,900 \mathrm{~cm}-1$ (red) and $23,100 \mathrm{~cm}-1$ (blue) components of the Cu-MOR Abs feature during reaction with methane. Adapted from reference 134 with permission. Copyright 2015, American Chemical Society.

feature shown in figure 34. One of these components (MOR 1, red) has reactivity comparable to ZSM-5, while the other (MOR 2, blue) exhibits a significantly lower activation barrier (by 3-4 kcal/mol) and significantly higher entropy of activation (by 8-9 EU). This showed $\mathrm{Cu} / \mathrm{O}$ active sites with perturbed reactivity exist in Cu-MOR. ${ }^{134}$

Resonance Raman studies summarized in figure 35 were preformed to determine the origin of this difference in reactivity. ${ }^{134}$ From data shown in figure 33 , the $22,000 \mathrm{~cm}^{-1}$ Abs feature was resolved into two components with absorption maxima at 21,900 and 23,100 associated with MOR 1 and MOR 2, respectively. Tuning a laser into the $22,000 \mathrm{~cm}^{-1}$ Abs feature also results in resonance enhancement of vibrations associated with both components. As shown in figure 35, the $r R$ spectrum of $\mathrm{Cu}-\mathrm{MOR}$ strongly resembles that of Cu-ZSM-5. However some features in the Cu-MOR spectrum are clearly split into two components (see marked peaks in figure 35) - consistent with 
two types of $\left[\mathrm{Cu}_{2} \mathrm{O}\right]^{2+}$ active sites in $\mathrm{Cu}-\mathrm{MOR}$.

Since MOR 1 reacts more rapidly at $200 \stackrel{\circ}{C}$ (due to the smaller entropic contribution to the Gibbs free energy of activation), it was possible to enrich samples in MOR 2, and this enabled assignment of the rR features of each component. This showed the rR features and Abs features of MOR 1 and MOR 2 are highly similar to each other, as well as to Cu-ZSM-5. Thus from Abs and rR spectroscopy, the three $\left[\mathrm{Cu}_{2} \mathrm{O}\right]^{2+}$ active sites have highly similar geometric and electronic structures. ${ }^{37,134}$

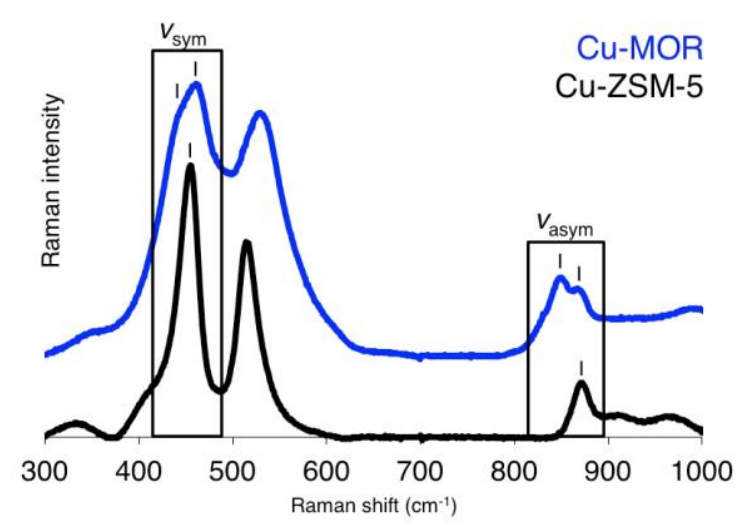

\begin{tabular}{rccc} 
& ZSM-5 & MOR 1 & MOR 2 \\
\hline Abs feature $\left(\mathrm{cm}^{-1}\right)$ & 22,700 & 21,900 & 23,100 \\
$v_{\text {sym }}\left(\mathrm{cm}^{-1}\right)$ & 456 & 465 & 450 \\
$V_{\text {asym }}\left(\mathrm{cm}^{-1}\right)$ & 870 & 850 & 870 \\
$\Delta \mathrm{H}^{\ddagger}(\mathrm{kcal} / \mathrm{mol})$ & 15.7 & 14.7 & 11.1 \\
$\Delta \mathrm{S}^{\ddagger}(\mathrm{cal} / \mathrm{mol} \cdot \mathrm{K})$ & -35 & -36 & -44
\end{tabular}

Figure 35. Comparison of $r R$ spectra from $\mathrm{Cu}$ MOR (blue) and Cu-ZSM-5 (black). Peaks corresponding to $v_{\text {sym }}$ and $v_{\text {asym }}$ are indicated, showing two components are present in $\mathrm{Cu}$ MOR. The table summarizes the spectroscopy and reactivity of $\left[\mathrm{Cu}_{2} \mathrm{O}\right]^{2+}$ cores in $\mathrm{Cu}-\mathrm{ZSM}-5$ and Cu-MOR.

The differences in geometric and electronic structure defined experimentally across the $\left[\mathrm{Cu}_{2} \mathrm{O}\right]^{2+}$ cores in $\mathrm{Cu}-\mathrm{ZSM}-5$ and $\mathrm{Cu}-\mathrm{MOR}$ are too subtle to explain the 25$30 \%$ reduction in activation barrier of MOR 2 . This indicates there is a contribution to reactivity that does not manifest in the ground state spectral features of MOR 2. The differential reactivity of MOR 2 is therefore ascribed to a second-sphere effect, however the precise nature of this interaction is not yet understood. ${ }^{134}$ This raises a parallel to biology, where for example the coupled binuclear $\mathrm{Cu}$ enzymes (i.e. hemocyanin, tyrosinase, catechol oxidase, and $\mathrm{NspF}$ ) carry out diverse functions ranging from $\mathrm{O}_{2}$ binding to aromatic hydroxylation using $\left[\mathrm{Cu}_{2}\left(\mathrm{O}_{2}\right)\right]^{2+}$ intermediates with highly similar geometric and electronic structures (see 6.3 for detail). ${ }^{18}$ 
4.3 - Activation of $\mathrm{O}_{2}$. The ability of $\mathrm{Cu}$ zeolites to activate atmospheric $\mathrm{O}_{2}$ for selective oxidation chemistry is highly significant, as this is the most appealing oxidant for industrial-scale applications. Reduced $\mathrm{Cu}(\mathrm{I})-\mathrm{ZSM}-5$ and $\mathrm{Cu}(\mathrm{I})-\mathrm{MOR}$ bind and reduce $\mathrm{O}_{2}$ by four electrons during $\left[\mathrm{Cu}_{2} \mathrm{O}\right]^{2+}$ formation, ${ }^{36,37,134}$ and an oxygen precursor in the $\left[\mathrm{Cu}_{2} \mathrm{O}\right]^{2+}$ formation reaction

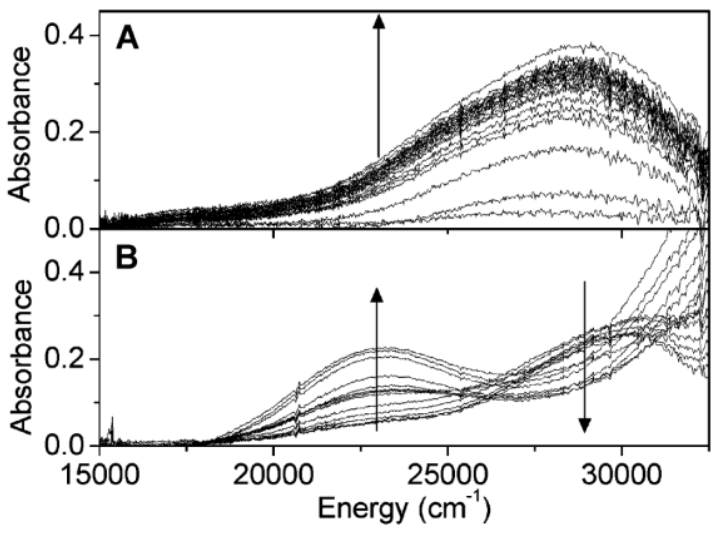

Figure 36. DR-UV-vis spectral changes during $\mathrm{O}_{2}$ activation of $\mathrm{Cu}(\mathrm{I})-\mathrm{ZSM}-5$ (top), and subsequent heating to form $\left[\mathrm{Cu}_{2} \mathrm{O}\right]^{2+}$ (bottom). Reproduced from reference ${ }^{136}$ with permission. Copyright 2010, American Chemical Society. has been trapped in Cu-ZSM-5. ${ }^{136}$ As shown in figure 36 (top), reacting $\mathrm{Cu}(\mathrm{I})-\mathrm{ZSM}-5$ with $\mathrm{O}_{2}$ at room temperature results in formation of an intense absorption feature at $29,000 \mathrm{~cm}^{-1}$. Upon heating, the $29,000 \mathrm{~cm}^{-1}$ absorption feature decays with the concomitant growth of the $22,700 \mathrm{~cm}^{-1}$ band of the $\left[\mathrm{Cu}_{2} \mathrm{O}\right]^{2+}$ intermediate (figure 36 , bottom).

Tuning a laser into the $29,000 \mathrm{~cm}^{-1}$ Abs band selectively resonance enhances vibrations of the oxygen precursor. ${ }^{136}$ The resulting resonance Raman spectrum in figure 37 (left, $\lambda_{\text {ex }}=364 \mathrm{~nm}$ ) shows an intense isotope insensitive vibration at $269 \mathrm{~cm}^{-1}$, as
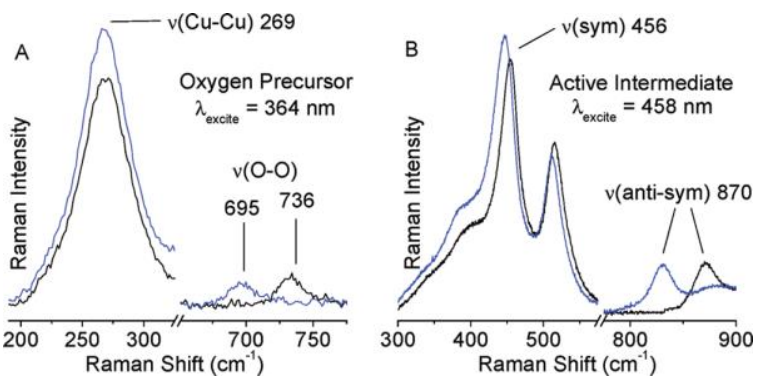

Figure 37. A) $r R$ data from $\mathrm{Cu}(\mathrm{I})-\mathrm{ZSM}-5$ activated at room temperature with ${ }^{16} \mathrm{O}_{2}$ (black) and ${ }^{18} \mathrm{O}_{2}$ (blue), and $\mathrm{B}$ ) after heating the oxygen intermediate to form $\left[\mathrm{Cu}_{2} \mathrm{O}\right]^{2+}$. Reproduced from reference ${ }^{136}$ with permission. Copyright 2010, American Chemical Society.

well as a weak feature at $736 \mathrm{~cm}^{-1}$ that shifts down by $41 \mathrm{~cm}^{-1}$ with ${ }^{18} \mathrm{O}_{2}$. These $\mathrm{rR}$ features decay upon heating, and shifting to $458 \mathrm{~nm}$ laser excitation, the resonance 
Raman vibrations of the $\left[\mathrm{Cu}_{2} \mathrm{O}\right]^{2+}$ intermediate grow in (figure 37, right). The above Abs and $364 \mathrm{~nm} \mathrm{rR}$ features are therefore correlated with the oxygen intermediate, which converts directly to $\left[\mathrm{Cu}_{2} \mathrm{O}\right]^{2+}$.

The $\mathrm{rR}$ and Abs spectral features of the oxygen precursor in Cu-ZSM-5 are characteristic of $\mu-\eta^{2}: \eta^{2}$ peroxo-bridged dicopper sites (see table 5). The $29,000 \mathrm{~cm}^{-1}$ Abs band was therefore assigned as an $\mathrm{O}_{2}{ }^{2-}\left(\pi^{*}{ }_{\sigma} \rightarrow \mathrm{Cu}(\mathrm{II})\right.$ charge transfer transition, while the $269 \mathrm{~cm}^{-1}$ and $736 \mathrm{~cm}^{-1}$ features in the resonance Raman spectrum are assigned as the $\mathrm{Cu}-\mathrm{Cu}$ stretching mode and $\mathrm{O}-\mathrm{O}$ stretch (respectively) of a $\left[\mathrm{Cu}_{2}\left(\mathrm{O}_{2}\right)\right]^{2+}$ core. $\left[\mathrm{Cu}_{2}\left(\mathrm{O}_{2}\right)\right]^{2+}$ cores with highly similar spectral features are stabilized in the coupled binuclear $\mathrm{Cu}$ enzymes (i.e. hemocyanin, tyrosinase, catechol oxidase, and NspF discussed in section 6.1). ${ }^{18}$

Spectral changes in Abs and $\mathrm{rR}$ that occur upon heating demonstrate this $\left[\mathrm{Cu}_{2}\left(\mathrm{O}_{2}\right)\right]^{2+}$ site converts directly into the $\left[\mathrm{Cu}_{2} \mathrm{O}\right]^{2+}$ intermediate. ${ }^{136} \mathrm{O}_{2}$ temperatureprogrammed desorption studies were performed to clarify the mechanism of this conversion - in particular, the fate of the

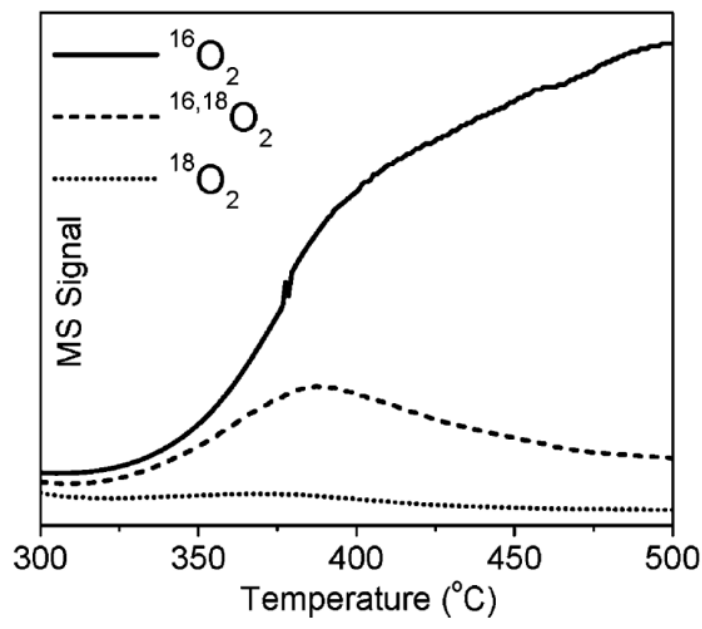

second $\mathrm{O}$ atom of the $\left[\mathrm{Cu}_{2}\left(\mathrm{O}_{2}\right)\right]^{2+}$ precursor. Figure 38. $\mathrm{O}_{2}$ temperature programmed desorption quantifying release of $\mathrm{O}_{2}$ isotopomers Heating activated Cu-ZSM-5 leads to during $\left[\mathrm{Cu}_{2}{ }^{18} \mathrm{O}\right]^{2+}$ desorption. Reproduced from desorption of the bridging oxo of the $\left[\mathrm{Cu}_{2} \mathrm{O}\right]^{2+}$ reference ${ }^{136}$ with permission. Copyright 2010, American Chemical Society.

core, forming the reduced $2 \mathrm{Cu}(\mathrm{I})$ active site and releasing $\mathrm{O}_{2}$. Through microscopic reversibility, the molecular-level details of this process relate directly to the mechanism 
of O-atom loss from the peroxide intermediate. As shown in figure $38, \mathrm{O}_{2}$ TPD of ${ }^{18} \mathrm{O}_{2}$ activated Cu-ZSM-5 results in release of three isotopomers $-{ }^{16} \mathrm{O}_{2},{ }^{16 / 18} \mathrm{O}_{2}$, and ${ }^{18} \mathrm{O}_{2}$. Since ${ }^{18} \mathrm{O}_{2}$ activation is known to produce fully-labeled $\left[\mathrm{Cu}_{2}{ }^{18} \mathrm{O}\right]^{2+}$, only the ${ }^{16 / 18} \mathrm{O}_{2}$ and ${ }^{18} \mathrm{O}_{2} \mathrm{MS}$ signals reflect desorption of $\left[\mathrm{Cu}_{2}{ }^{18} \mathrm{O}\right]^{2+}$. As the core desorbs between $350-420$ ${ }^{\circ} \mathrm{C}$, the ratio of ${ }^{16 / 18} \mathrm{O}_{2}:{ }^{18} \mathrm{O}_{2}$ released is greater than $10: 1$. This indicates most $\left[\mathrm{Cu}_{2}{ }^{18} \mathrm{O}\right]^{2+}$ recombines with a ${ }^{16} \mathrm{O}$ atom, and not an ${ }^{18} \mathrm{O}$ atom (derived from $\left.\left[\mathrm{Cu}_{2}\left({ }^{18} \mathrm{O}_{2}\right)\right]^{2+}\right)$. The isotope scrambling observed in $\mathrm{O}_{2}$ TPD therefore reflects the ability of the zeolite lattice to act as a reservoir of mobile $\mathrm{O}$ atoms. ${ }^{144-146}$ This reservoir includes ${ }^{18} \mathrm{O}$ atoms derived from the $\left[\mathrm{Cu}_{2}\left({ }^{18} \mathrm{O}_{2}\right)\right]^{2+}$ oxygen precursor, but from experiment the ratio of ${ }^{16} \mathrm{O}:{ }^{18} \mathrm{O}$ in this O-atom reservoir is greater than $10: 1$. The ability of the zeolite lattice to accept exogenous atoms is therefore critical to the conversion of $\left[\mathrm{Cu}_{2}\left(\mathrm{O}_{2}\right)\right]^{2+}$ into $\left[\mathrm{Cu}_{2} \mathrm{O}\right]^{2+} \cdot{ }^{136}$ 
The conversion of $\left[\mathrm{Cu}_{2}\left(\mathrm{O}_{2}\right)\right]^{2+}$ into $\left[\mathrm{Cu}_{2} \mathrm{O}\right]^{2+}$ requires two additional electrons to cleave the $\mathrm{O}-\mathrm{O}$ bond and liberate $\mathrm{O}^{2-}$. Oxo loss from $\left[\mathrm{Cu}_{2}\left(\mathrm{O}_{2}\right)\right]^{2+}$ would form a $\left[\mathrm{Cu}(\mathrm{III})_{2} \mathrm{O}\right]^{4+}$ site that is predicted by DFT to be electronically unstable, oxidizing the zeolite lattice to form $\left[\mathrm{Cu}(\mathrm{II})_{2} \mathrm{O}\right]^{2+} .{ }^{37}$ The capacity of the zeolite lattice to serve as an

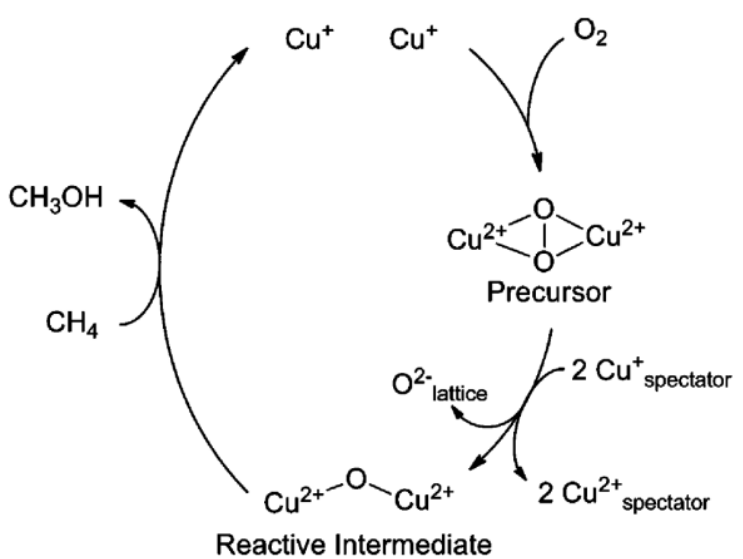

Figure 39. Proposed reaction cycle for $\mathrm{O}_{2}$ activation and $\mathrm{CH}_{4}$ hydroxylation by $\mathrm{Cu}$-zeolites. Reproduced from reference ${ }^{136}$ with permission. Copyright 2010, American Chemical Society.

electron donor/acceptor has been

established, ${ }^{147-149}$ indicating the electron holes generated by $\left[\mathrm{Cu}(\mathrm{II})_{2} \mathrm{O}\right]^{2+}$ formation can propagate over significant distances to spectator $\mathrm{Cu}(\mathrm{I})$ sites. Thus spectator $\mathrm{Cu}(\mathrm{I})$ sites would provide the two exogenous electrons required to cleave the O-O bond to form $\left[\mathrm{Cu}_{2} \mathrm{O}\right]^{2+}$ during $\mathrm{O}_{2}$ activation of $\mathrm{Cu}$-zeolites. This has led to the model of active core formation summarized in figure $39 .{ }^{136}$ In this model, $\mathrm{O}_{2}$ binds to a reduced $2 \mathrm{Cu}(\mathrm{I})$ active site, which reduces $\mathrm{O}_{2}$ by two electrons to form a $\left[\mathrm{Cu}_{2}\left(\mathrm{O}_{2}\right)\right]^{2+}$ oxygen precursor. Upon heating, spectator $\mathrm{Cu}(\mathrm{I})$ centers provide two additional electrons to cleave the $\mathrm{O}-\mathrm{O}$ bond. This results in formation of the $\left[\mathrm{Cu}_{2} \mathrm{O}\right]^{2+}$ reactive intermediate, as well as $\mathrm{Cu}(\mathrm{II})$ spectator sites, and an oxo that enters the pool of 'mobile' lattice $O$ atoms (vide supra). Methane then in principle reduces the active site by two electrons, forming methanol, which is released to return the active site to its reduced $2 \mathrm{Cu}(\mathrm{I})$ state. These steps subsequent to $\mathrm{H}$-atom abstraction still need to be explored as does the role of spectator $\mathrm{Cu}$ in the conversion of $\left[\mathrm{Cu}_{2}\left(\mathrm{O}_{2}\right)\right]^{2+}$ to $\left[\mathrm{Cu}_{2} \mathrm{O}\right]^{2+}$. 
Recently, an oxygen intermediate has also been identified in Cu-SSZ-13 using Raman spectroscopy. ${ }^{150}$ In this system, $\mathrm{O}_{2}$ activation is correlated with the appearance of absorption and Raman features characteristic of a $\mu-1,2$-peroxide-bridged $2 \mathrm{Cu}$ (II) site (see table 5). In the same study, Raman and absorption features assigned to a $\left[\mathrm{Cu}_{2} \mathrm{O}\right]^{2+}$ core with an acute bridge angle of ca. 100 were reported. It will be interesting to evaluate whether this end-on-bridged peroxide intermediate converts to the $\left[\mathrm{Cu}_{2} \mathrm{O}\right]^{2+}$ core, and once quantitative reactivity data on this acute $\left[\mathrm{Cu}_{2} \mathrm{O}\right]^{2+}$ site become available, it will be also interesting to see how this change in geometry alters the reactivity of the $\left[\mathrm{Cu}_{2} \mathrm{O}\right]^{2+}$ core.

4.4 - Activation of $\mathrm{N}_{2} \mathrm{O}$. In addition to $\mathrm{O}_{2}$, the $2 \mathrm{Cu}(\mathrm{I})$ active sites of $\mathrm{Cu}-\mathrm{ZSM}-5$ and $\mathrm{Cu}-$ MOR also activate $\mathrm{N}_{2} \mathrm{O}$, forming $\left[\mathrm{Cu}_{2} \mathrm{O}\right]^{2+}$ intermediates through $\mathrm{O}$-atom transfer with release of $\mathrm{N}_{2} .^{37,134,135}$ In contrast to O-O bond cleavage of $\mathrm{O}_{2}$, which requires four electrons,

\begin{tabular}{|c|c|c|c|c|c|}
\hline & \multicolumn{3}{|c|}{ oxidized form $\left(\left[\mathrm{Cu}_{2} \mathrm{O}\right]^{2+}\right)$} & \multicolumn{2}{|c|}{$\begin{array}{l}\text { reduced form } \\
\left(\mathrm{Cu}^{\mathrm{I}} \cdots \mathrm{Cu}^{\mathrm{I}}\right)\end{array}$} \\
\hline & $\begin{array}{l}\angle \mathrm{Cu}-\mathrm{O}-\mathrm{Cu} \\
(\mathrm{deg})\end{array}$ & $\begin{array}{c}\nu_{\text {symm }} / \nu_{\text {antisym }} \\
\left(\mathrm{cm}^{-1}\right)\end{array}$ & $\begin{array}{c}\mathrm{CT}_{\text {oxo to }} \mathrm{Cu} \\
\left(\mathrm{cm}^{-1}\right)\end{array}$ & $\begin{array}{l}\text { binding } \\
\text { types }\end{array}$ & $d_{\mathrm{Cy}}(\AA) \mathrm{Cu}$ \\
\hline exp. & 139 & $456 / 870$ & 22700 & $\begin{array}{c}2 \text { or } 3 \\
\text { CN }\end{array}$ & 4.43 \\
\hline site 1 & 134 & $445 / 855$ & 23159 & II,III & 4.56 \\
\hline site 2 & 143 & $426 / 857$ & 23502 & II,III & 5.03 \\
\hline site 3 & 146 & $407 / 784$ & 21693 & $\mathrm{I}, \mathrm{I}$ & 4.14 \\
\hline site 4 & 144 & $417 / 850$ & 22280 & II,III & 5.02 \\
\hline site 5 & 133 & $447 / 859$ & 22617 & $\mathrm{I}, \mathrm{I}$ & 2.48 \\
\hline site 6 & 135 & $451 / 842$ & 22926 & III,III & 4.17 \\
\hline site 7 & 145 & $415 / 856$ & 21300 & II,III & 5.85 \\
\hline site 8 & 147 & $391 / 860$ & 22841 & I,III & 3.54 \\
\hline site 9 & 145 & $419 / 843$ & 21077 & $\mathrm{I}, \mathrm{III}$ & 3.57 \\
\hline site 10 & 145 & $408 / 815$ & 22897 & $\mathrm{I}, \mathrm{III}$ & 4.76 \\
\hline
\end{tabular}
only two electrons are required to cleave the $\mathrm{N}-\mathrm{O}$ bond of $\mathrm{N}_{2} \mathrm{O}$. In this case, the $2 \mathrm{Cu}(\mathrm{l})$ active site provides both electrons directly, Table 6. Predicted geometric structures and spectroscopic features of the ten $\left[\mathrm{Cu}_{2} \mathrm{O}\right]^{2+}$ and $2 \mathrm{Cu}(\mathrm{I})$ active sites stabilized in a $10 \mathrm{MR}$ of ZSM5. Reproduced from reference 135 with permission. Copyright 2014, American Chemical Society.

and exogenous electrons from spectator sites

are not required (unlike $\mathrm{O}_{2}$ activation, vide supra). To define the geometric and electronic structural requirements for $\mathrm{N}_{2} \mathrm{O}$ activation by Cu-zeolites, DFT models were constructed of $\left[\mathrm{Cu}_{2} \mathrm{O}\right]^{2+}$ intermediates stabilized by each of the ten possible Al-O-Si-O- 
Si-O-Al sequences in a 10-membered ring of the ZSM-5 lattice (see figure $31 \mathrm{~A}$, right). ${ }^{135}$ These all feature three-coordinate $\mathrm{Cu}(\mathrm{II})$ centers, each ligated by two lattice oxygen atoms derived from an Al T-site, as well as the bridging oxo ligand. The geometric structures and predicted spectroscopic features $(\mathrm{Abs} / \mathrm{rR})$ of the ten $\left[\mathrm{Cu}_{2} \mathrm{O}\right]^{2+}$ models are given in table 6 , and these are highly similar across the ten models. The corresponding $2 \mathrm{Cu}(\mathrm{I})$ sites were generated by deleting the bridging $\mathrm{O}$ atoms of $\left[\mathrm{Cu}_{2} \mathrm{O}\right]^{2+}$ cores and geometry optimizing the resulting structures. There is significant variability in the $\mathrm{Cu}(\mathrm{I})$ coordination environment across the ten $2 \mathrm{Cu}(\mathrm{I})$ models (see table 6 and figure $40 \mathrm{~b}$ ), and this leads to a large spread in relative stabilities of the $2 \mathrm{Cu}(\mathrm{I})$ sites $(>40 \mathrm{kcal} / \mathrm{mol}$, see figure $40 \mathrm{a}$, left). This is in contrast to the $\left[\mathrm{Cu}_{2} \mathrm{O}\right]^{2+}$ models, where their similar geometric structures lead to a small spread in relative energies (15 kcal/mol, figure $40 \mathrm{a}$, right). It was also found that $2 \mathrm{Cu}(\mathrm{I})$ sites with approximately linear $\mathrm{Cu}(\mathrm{I})$ coordination were found to be most stable, while two- and threecoordinate $\mathrm{Cu}(\mathrm{I})$ structures featuring more acute $\mathrm{O}-\mathrm{Cu}-\mathrm{O}$ angles were destabilized (figure 40b). ${ }^{135}$

The most stable $\left[\mathrm{Cu}_{2} \mathrm{O}\right]^{2+}$ and $2 \mathrm{Cu}(\mathrm{l})$ sites (site 6 in table 6) correspond to the active site discussed in 4.1 , which was initially identified as a candidate structure based on results from X-ray 2014, American Chemical Society.

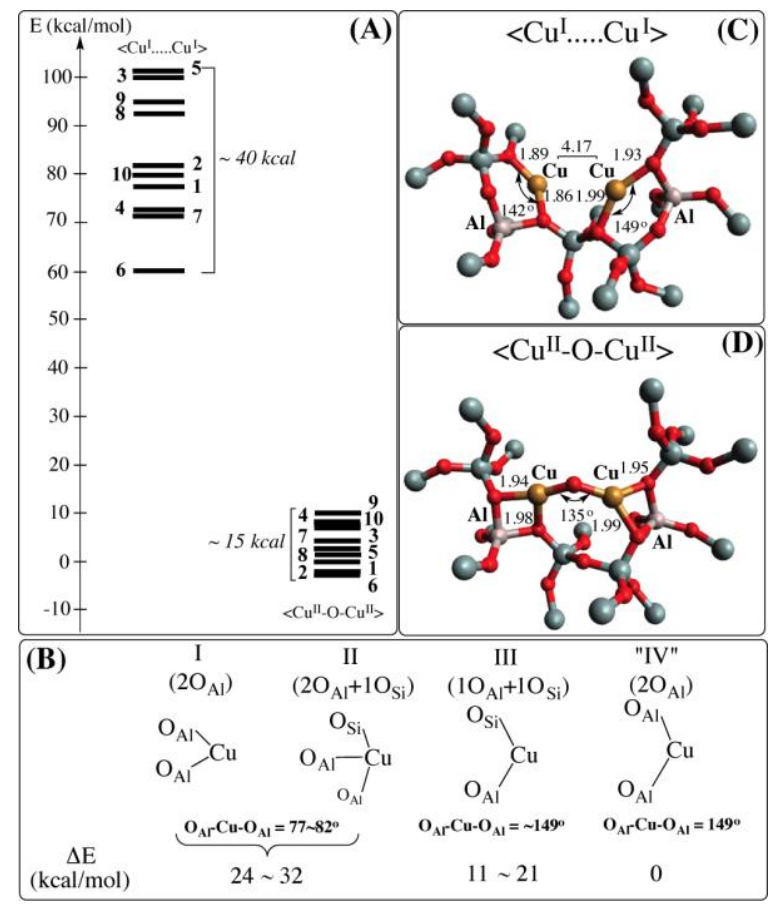

Figure 40. A) relative energies of the reduced and oxidized active site DFT models and B) their $\mathrm{Cu}(\mathrm{I})$ coordination modes. Structures of the reduced $(C)$ and oxidized (D) model assigned to the active site seen in experiment. Si atoms are colored dark grey, Al atoms white. Reproduced from reference ${ }^{135}$ with permission. Copyright 

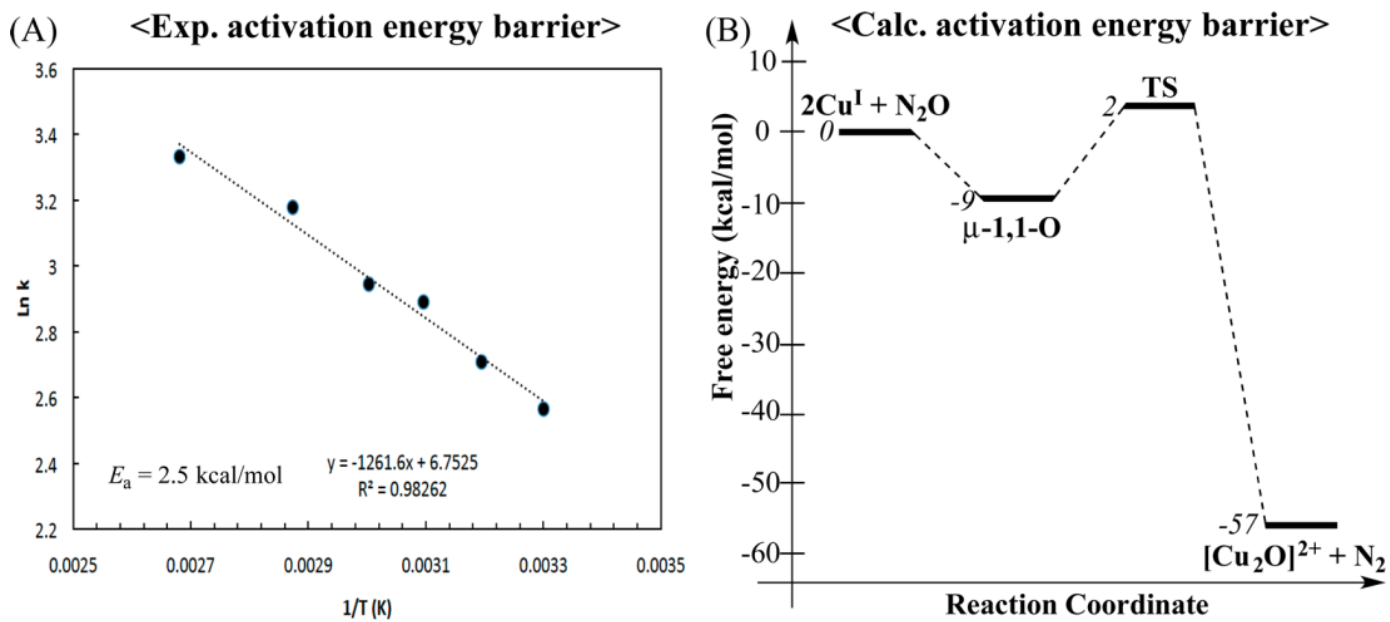

Figure 41. A) Arrhenius plot for $\mathrm{N}_{2} \mathrm{O}$ activation by $\mathrm{Cu}(\mathrm{I})-\mathrm{ZSM}-5$ to form $\left[\mathrm{Cu}_{2} \mathrm{O}\right]^{2+}$ and $\mathrm{B}$ ) summary of DFT reaction coordinate for $\mathrm{N}_{2} \mathrm{O}$ activation. Reproduced from reference ${ }^{135}$ with permission. Copyright 2014 American Chemical Society.

diffraction. ${ }^{37,135,143}$ This $2 \mathrm{Cu}(\mathrm{I})$ site is at least $12 \mathrm{kcal} / \mathrm{mol}$ more stable than the others, and the corresponding $\left[\mathrm{Cu}_{2} \mathrm{O}\right]^{2+}$ accurately reproduces the spectral features of the reactive intermediate defined experimentally (see 4.1). ${ }^{37,135}$ Based on this, the $\left[\mathrm{Cu}_{2} \mathrm{O}\right]^{2+}$ site observed experimentally was assigned to site 6 , and the reduced and oxidized structures of this site are shown in figure $40 \mathrm{c}$ and $40 \mathrm{~d}$, respectively. ${ }^{135}$ In the reduced site, both $\mathrm{Cu}(\mathrm{I})$ centers are bound in an approximately linear fashion by one lattice $\mathrm{O}$ atom derived from an Al T-site, and another derived from the neighboring Si T-site (i.e. motif III in figure 40b). The Cu centers are separated by $4.17 \AA$, which is sufficiently short to enable bridging required for facile $\mathrm{N}_{2} \mathrm{O}$ reduction (vide infra). ${ }^{135}$

The reduced $2 \mathrm{Cu}(\mathrm{I})$ active site model was used to evaluate the mechanism of $\mathrm{N}_{2} \mathrm{O}$ activation by $\mathrm{Cu}$ -

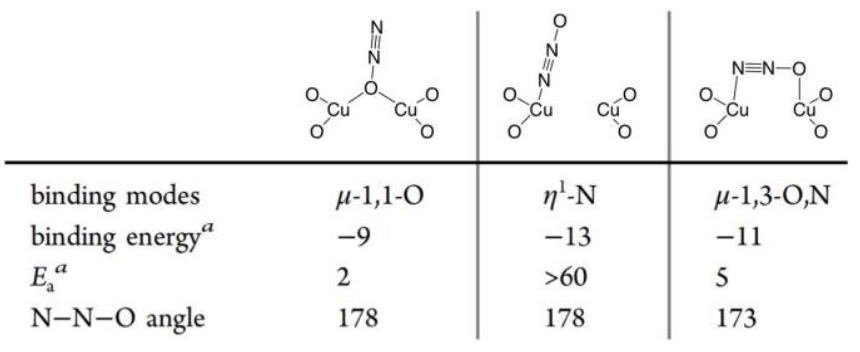
ZSM-5. ${ }^{135}$ First, the kinetics of $\mathrm{N}-\mathrm{O}$ Figure 42. Binding modes of $\mathrm{N}_{2} \mathrm{O}$, their associated cleavage were evaluated for $\mathrm{N}-\mathrm{O}$ cleavage. a given in $\mathrm{kcal} / \mathrm{mol}$ 
experimentally by tracking the growth of the $22,700 \mathrm{~cm}^{-1}$ during reaction of $\mathrm{Cu}(\mathrm{I})-\mathrm{ZSM}-5$ with $\mathrm{N}_{2} \mathrm{O}$. The resulting Arrhenius plot shown in figure $41 \mathrm{~A}$ gave an apparent activation barrier $E_{\mathrm{a}}=2.5 \mathrm{kcal} / \mathrm{mol}$. Note that this apparent barrier includes the enthalpy of $\mathrm{N}_{2} \mathrm{O}$ adsorption, and is therefore reduced from the value for $\mathrm{N}-\mathrm{O}$ cleavage. As shown in figure $42, \mathrm{~N}_{2} \mathrm{O}$ can bind in three ways to the $2 \mathrm{Cu}(\mathrm{I})$ active site. In all cases, $\mathrm{N}_{2} \mathrm{O}$ binding is exothermic by $9-13 \mathrm{kcal} / \mathrm{mol}$. While monohaptic binding through the terminal nitrogen to a single $\mathrm{Cu}(\mathrm{I})$ center is most stable, there is no reaction coordinate for O-atom transfer resulting in a $\left[\mathrm{Cu}_{2} \mathrm{O}\right]^{2+}$ for this intermediate. The $\mu-1,1-\mathrm{O}$ and $\mu-1,3-\mathrm{O}, \mathrm{N}$ structures in which $\mathrm{N}_{2} \mathrm{O}$ bridges adjacent $\mathrm{Cu}(\mathrm{I})$ centers, on the other hand, lead to low apparent barriers $(2-5 \mathrm{kcal} / \mathrm{mol})$ that are consistent with experiment $(2.5 \mathrm{kcal} / \mathrm{mol}) .{ }^{135}$

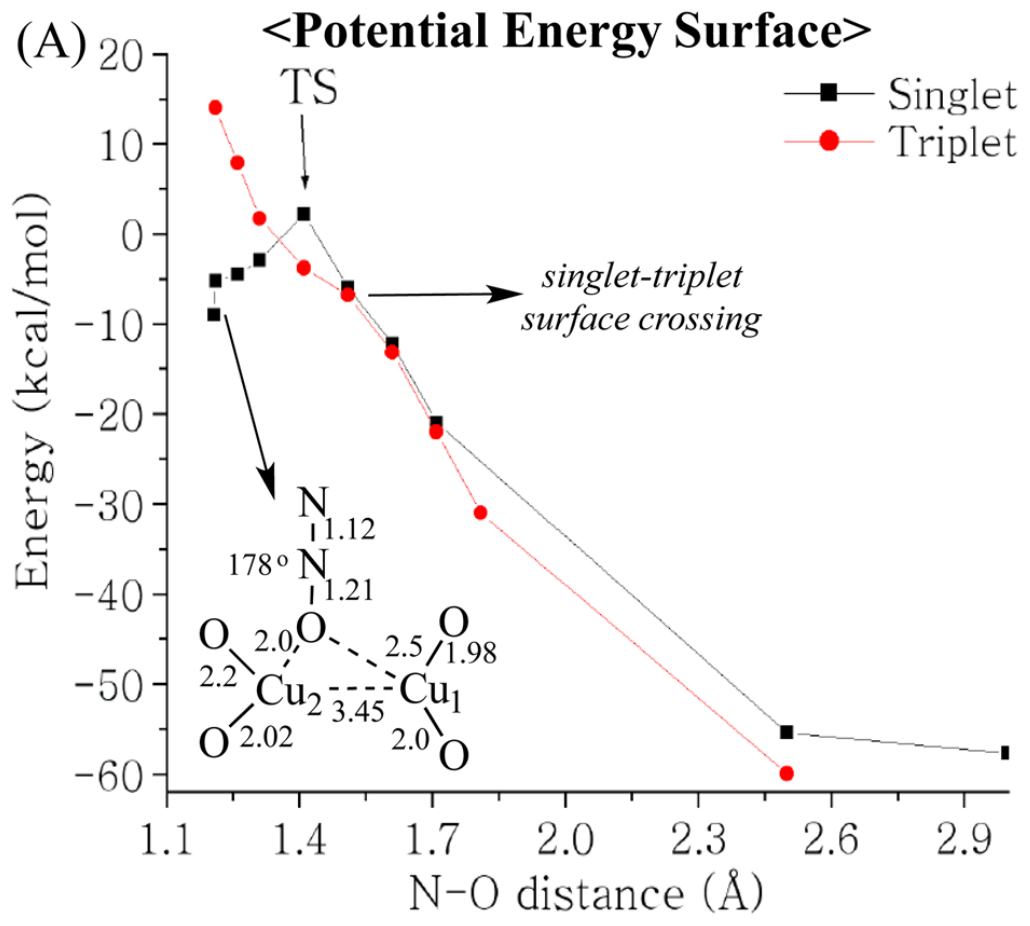

(B) <TS-Geometry>
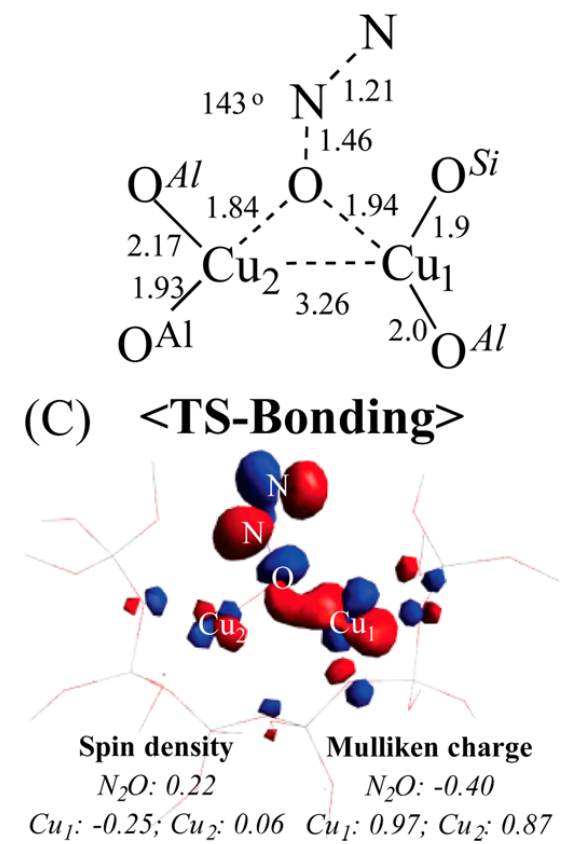

Figure 43. A) Singlet (black) and triplet (red) potential energy surfaces associated with $\mathrm{N}-\mathrm{O}$ elongation. B) Geometric structure of the N-O cleavage transition state, with the associated electronic structure (C) showing delocalization of the $\mathrm{N}_{2} \mathrm{O} \pi^{*}$ LUMO into the $\mathrm{Cu}$ centers of the active site. Reproduced from reference ${ }^{135}$ with permission. Copyright 2014, American Chemical Society. 
The $\mu-1,1-\mathrm{O}$ intermediate leads to the lowest barrier for $\mathrm{N}-\mathrm{O}$ cleavage (figures 41B, 42). ${ }^{135}$ As shown in figure $43 \mathrm{~A}$, the transition state for $\mathrm{N}-\mathrm{O}$ cleavage lies on the singlet surface, with the $\mathrm{N}-\mathrm{O}$ bond elongated from its equilibrium length of $1.21 \AA$ to $1.46 \AA$. At the transition state geometry (shown in figure 43B), the $\mathrm{N}-\mathrm{N}-\mathrm{O}$ angle is no longer linear, but instead bent to $143^{\circ}$. The electronic structure associated with this geometry is shown in figure $43 \mathrm{C}$, where the $\pi^{*} \mathrm{LUMO}$ of $\mathrm{N}_{2} \mathrm{O}$ is significantly delocalized onto one of the $\mathrm{Cu}$ centers. This reflects transfer of a single electron from $\mathrm{Cu}_{1}$ to $\mathrm{N}_{2} \mathrm{O}$ at the transition state. With further $\mathrm{N}-\mathrm{O}$ elongation past the transition state, the second electron is transferred from $\mathrm{Cu}_{2}$, completing the reaction and releasing $\mathrm{N}_{2}$. Two factors contribute to the low barrier for $\mathrm{N}-\mathrm{O}$ cleavage. First, the $2 \mathrm{Cu}(\mathrm{l})$ site has a sufficiently short $\mathrm{Cu}$... $\mathrm{Cu}$ separation to enable $\mathrm{N}_{2} \mathrm{O}$ to bridge adjacent $\mathrm{Cu}(\mathrm{I})$ centers. This geometric structure provides a direct orbital pathway for the sequential two-electron reduction of $\mathrm{N}_{2} \mathrm{O}$, whereby each $\mathrm{Cu}(\mathrm{l})$ center donates a single electron to cleave the $\mathrm{N}$ $\mathrm{O}$ bond. On the other hand, for longer $\mathrm{Cu}$...Cu distances where $\mathrm{N}_{2} \mathrm{O}$ cannot bridge the $\mathrm{Cu}(\mathrm{I})$ centers, there is no such orbital pathway for transfer of the second electron and this results in a $14 \mathrm{kcal} / \mathrm{mol}$ higher barrier for $\mathrm{N}-\mathrm{O}$ cleavage. Second, the formation of two strong $\mathrm{Cu}(\mathrm{II})$-oxo bonds during O-atom transfer provides the strong driving force for $\mathrm{N}-\mathrm{O}$ cleavage, tuning down the reaction barrier. ${ }^{135}$ In nature, the same reaction is also performed with a low barrier using a multinuclear $\mathrm{Cu}$ center - the $\mathrm{Cu}_{z}$ cluster of nitrous oxide reductase. ${ }^{151}$ Parallels and differences between $\mathrm{Cu}_{z}$ and the $\mathrm{Cu}(\mathrm{I})$-zeolites are discussed in section 6.1 .

4.5 - Other $\mathrm{Cu}_{x} \mathrm{O}_{y}$ Active Sites of Methane Hydroxylation. Thus far, the $\left[\mathrm{Cu}_{2} \mathrm{O}\right]^{2+}$ sites in MFI and MOR (vide supra) are the only active Cu-O species identified in zeolites 
with site selective spectroscopy. However, other reactive $\mathrm{Cu}-\mathrm{O}$ species of varying nuclearity and with variable activation and reaction constraints have been proposed. ${ }^{36,66,77,152,153}$ This is indicated by the extraction of additional methanol after high temperature activation with $\mathrm{O}_{2}$ or $\mathrm{N}_{2} \mathrm{O}$ and methane reaction at $\geq 473 \mathrm{~K}$, or at elevated pressure, in several copper-zeolites (e.g. FER, SSZ-13, SSZ-16, SSZ-39, MOR, ZSM-5, BEA, FAU) and zeotypes (SAPO-34) 66,84,152,154,155 which lack known spectroscopic features. Only recently, anaerobic reoxidation of the active site with $\mathrm{H}_{2} \mathrm{O}$ was observed, forming $\mathrm{H}_{2}$ as a side product. ${ }^{156}$ The clarification of the unknown active site structures and reactivity is a work in progress, and an overview will be given in this section. The interest in these active sites is linked to their attractive properties from an engineering point of view. Tomkins et al, for example, detected methanol (up to 55.4 $\mu \mathrm{mol} / \mathrm{g}$ ) upon steaming of over-exchanged Cu-ZSM-5, Cu-MOR and $\mathrm{Cu}-\mathrm{Y}$ after $\mathrm{O}_{2}$ treatment at $200^{\circ} \mathrm{C}$ and methane reaction at $200^{\circ} \mathrm{C}$ and 36 bar. ${ }^{155,157}$ The isothermal conditions of oxidation and methane activation, as well as the consistent yield in subsequent reaction cycles are promising characteristics for industrial applications.

Influential parameters in obtaining different active sites are the zeolite topology, aluminium distribution and counterbalancing cations, exchange conditions $(\mathrm{pH}$, concentration, salt precursor) and total copper loading. These are discussed in sections 2.2-2.5. Copper speciation is furthermore dependent on environmental conditions, such as temperature and atmosphere composition and pressure. ${ }^{76}$ Therefore, operando characterization techniques are imperative for reliable detection of active sites. Often, a multitude of reactive sites is encountered within the same material ${ }^{66,152,154}$, the majority 
of which lacks site-specific spectroscopy for unambiguous identification of the active sites.

A high spin density localized on the reactive oxygen consistently appears to be crucial in determining the reactivity of $\mathrm{Cu}$ active sites in zeolites. Also the stabilization of the oxidized product and confinement effects induced by the zeolite lattice play a role (vide infra). Extra-lattice oxygen sites with radical character in copper zeolites have already been described by the group of Ismagilov et al. in a 2002 study in the context of NO decomposition. ${ }^{158}$ They reported the formation of near-linear copper-oxygen chains in the channels of the MFI zeolite. Upon high temperature vacuum treatment, a distinctive EPR signal $\left(g_{\|}=2.02, g_{\perp}=2.05\right)$ lacking typical copper hyperfine splitting is formed and attributed to an anionic oxyl radical $\left[\mathrm{O}^{\cdot-}\right] .{ }^{159}$ The origin is proposed to be the autoreduction of linear $\left[\ldots-\mathrm{Cu}^{2+-} \mathrm{O}^{2-}-\ldots\right]$ chains in the zeolite channels to form a new mixed $\mathrm{Cu}^{2+} / \mathrm{Cu}^{+}$species with $\left[\ldots-\mathrm{Cu}^{+}-\mathrm{O}^{--}-\ldots\right]$. An absorption feature at $15800 \mathrm{~cm}^{-1}$ in the vacuum treated sample is linked to $\mathrm{Cu}^{2+}-\mathrm{Cu}^{+}$intervalence transitions (IVT) in the partially autoreduced copper oxide linear chains.

4.5.1 - Trinuclear $\left[\mathrm{Cu}_{3} \mathrm{O}_{3}\right]^{2+}$ Active Sites. The active site of particulate methane monooxygenase is presently defined as binuclear by the X-ray crystallography studies of Rosenzweig et al. ${ }^{160-162}$ However, due to challenges involving the instability of the enzyme in preparatory steps prior to analysis, arguments for a trimeric copper oxide cluster are postulated by Chan et al. ${ }^{163-166}$ Furthermore, A mononuclear active site resembling that of the lytic polysaccharide monooxygenases is also under 
consideration. ${ }^{21}$ In zeolites, a tricopper oxide species with high reactivity toward methane was recently proposed by Grundner et al..$^{55}$

Single-site tricopper species were synthesized in copper exchanged MOR and ZSM-5 67,79 zeolites by aqueous exchange at a controlled $\mathrm{pH}$ of 5,7 followed by activation at $450^{\circ} \mathrm{C}$ in $\mathrm{O}_{2}$ (section 2.4). A linear 1:3 relation of oxidised $\mathrm{CH}_{4}$ (calculated from the carbon balance in oxidised products $\mathrm{CH}_{3} \mathrm{OH}, \mathrm{CO}_{2}, \mathrm{CO}$ and $\left.\left(\mathrm{CH}_{3}\right)_{2} \mathrm{O}\right)$ versus the total copper content and a linear 2:3 ratio of consumed BAS per added copper is observed over a range of copper loadings on Cu-MOR (figure 44A). This is an indication for a single-site cationic cluster composed of three coppers per two exchanged protons, and is compatible with an extra-framework divalent tricopper cluster at a cation exchange site with two nearby lattice Al tetrahedra. A broad absorption band around $31000 \mathrm{~cm}^{-1}$
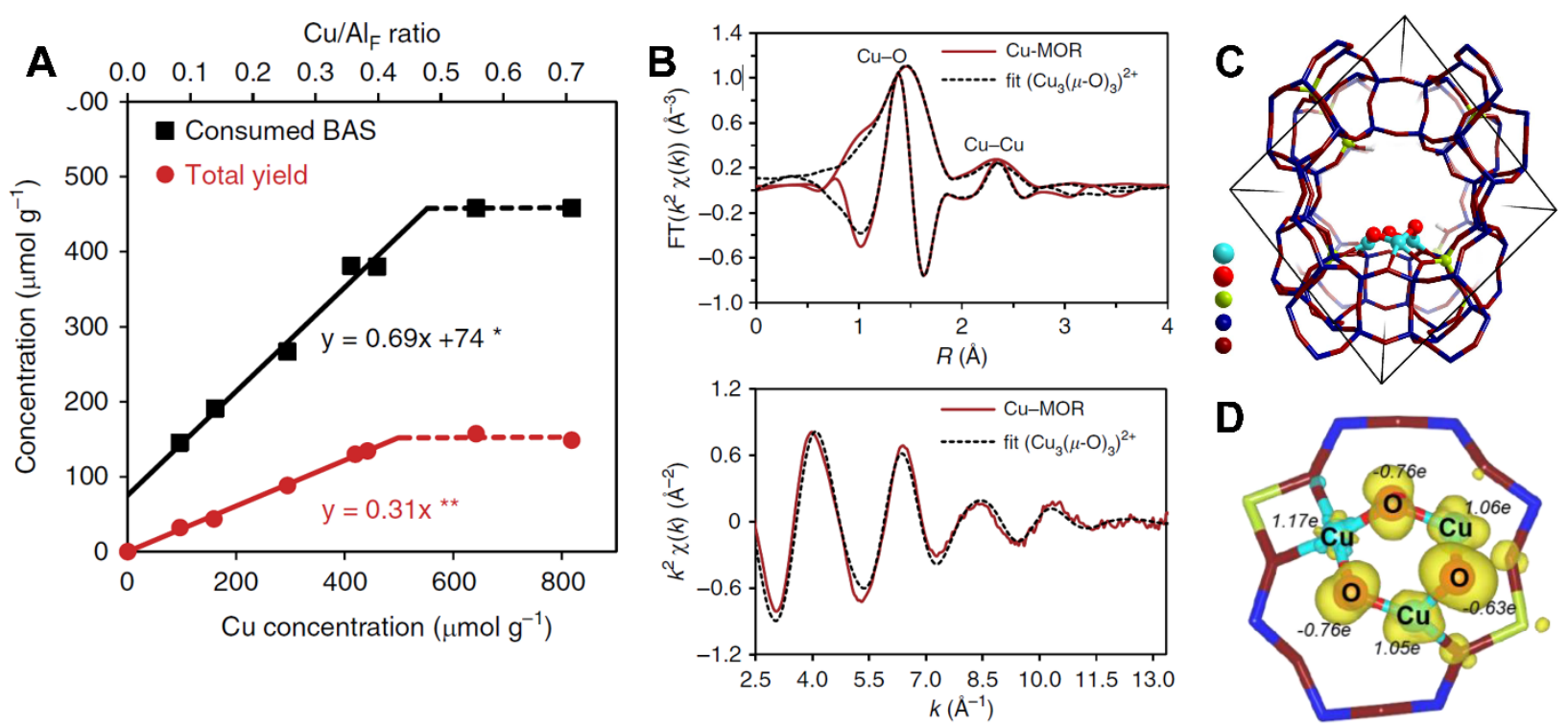

Figure 44. A) The relation between copper concentration in the MOR zeolite versus consumed BAS and the quantity of methane converted into oxygenated products $\left(\mathrm{CO}_{2}, \mathrm{CO}, \mathrm{CH}_{3} \mathrm{OH}\right.$ and $\left(\mathrm{CH}_{3}\right)_{2} \mathrm{O}$. B) The experimental EXAFS spectrum (red) and the EXAFS spectrum calculated for the model in D of this figure (black). C) the proposed location of the trinuclear copper cluster in the sidepocket of the MOR framework. D) The DFT model of the trinuclear copper cluster with Bader charges indicated. Al atoms are in yellow color, $\mathrm{Si}$ atoms in dark blue and $\mathrm{Cu}$ atoms in light blue. ${ }^{55}$ Adapted with permission from reference ${ }^{55}$. Copyright 2015 Nature Publishing Group 
in the UV-Vis spectrum has been linked to the tricopper site in the initial paper reporting the tricopper site, although without computational support. The band was observed to dissipate upon stoichiometric reaction with methane and to re-emerge upon reoxidation of the catalyst with $\mathrm{O}_{2 .}{ }^{55}$

A structure of the tricopper site was proposed by DFT simulation and is depicted in figure $44 \mathrm{C}, \mathrm{D}$. Formally, the charge on the atoms can be written as [Cu(II), $2 \mathrm{Cu}(\mathrm{III})$, $3 \mathrm{O}(-\mathrm{II})]^{2+}$ or $[3 \mathrm{Cu}(\mathrm{II}), 2 \mathrm{O}(-\mathrm{I}), \mathrm{O}(-\mathrm{II})]^{2+}$. The latter was preferred because it expresses the oxyl character of the extra-framework oxygen ions, proposed by Woertink et al. to be necessary in the transition state for methane $\mathrm{C}-\mathrm{H}$ activation by mono- $\mu$-oxo dicopper. ${ }^{37}$ This is also reflected by the spin densities of the calculated structure. ${ }^{55}$ The structure assignment is further supported by fitting the experimental Cu K-edge EXAFS spectrum of the material with the simulated spectrum of the $\left[\mathrm{Cu}_{3} \mathrm{O}_{3}\right]^{2+}$ (figure $44 \mathrm{~B}$ ). The EXAFS spectrum below $2 \AA$ can be fitted with two distinct $\mathrm{Cu}-\mathrm{O}$ metal-ligand bonds, corresponding to the Cu-OFramework and the Cu-OExtraframework bonds..$^{55,79}$

The assignment of the $\left[\mathrm{Cu}_{3} \mathrm{O}_{3}\right]^{2+}$ active site was based on sample averaging $\mathrm{X}-$ ray techniques and reactivity studies, using the reaction products of methane as a titrant for active site quantification (figure 44A). This method is reliant on the presence of a single site only. It is worthwhile to note that similar arguments were made in initial attempts to identify the $\alpha-\mathrm{Fe}$ site ${ }^{88}$, first identified by Panov et al., as a binuclear iron site. Per contra, thorough spectroscopic investigation recently showed this site to be mononuclear ${ }^{35}$ (see section 5). Site selective spectroscopy on the trinuclear cluster would circumvent the necessity of a single site and is required for the full characterization of the $\left[\mathrm{Cu}_{3} \mathrm{O}_{3}\right]^{2+}$ cluster in $\mathrm{Cu}-\mathrm{MOR}, \mathrm{Cu}-\mathrm{ZSM}-5$ and other materials. 
Moreover, no spectroscopic data in support of the absence of any spectator copper are given (e.g. UV-Vis, EPR). Additionally, $\mathrm{CO}_{2}$ and $\mathrm{CO}$ were observed in the reaction products, while the oxidation sites required to further oxidise the activated methane species to deeply oxidized $\mathrm{CO}$ and $\mathrm{CO}_{2}$ were not accounted for.

Finally, given the susceptibility of copper speciation to reaction conditions, operando techniques should be employed to ensure that the observed $\left[\mathrm{Cu}_{3} \mathrm{O}_{3}\right]^{2+}$ site, if present, is the actual active site at the basis of the catalytic function and not a precursor of, for example, a dinuclear active site formed under reducing $\mathrm{CH}_{4}$ atmosphere.

4.5.2 - Other $\mathrm{Cu}_{n} \mathrm{O}_{x}$ Copper Clusters, $\mathrm{n}>3$. Larger copper oxide clusters, with a nuclearity greater than three, have also been suggested as reactive sites. ${ }^{155}$ These clusters form within the zeolite micropores of over-exchanged Cu-zeolites by exchange of aqueous copper hydroxo oligomers with the zeolite Brönsted acid sites (Si(OH)Al) and silanol nests $(\mathrm{Si}(\mathrm{OH}))$. This process is influenced by solution $\mathrm{pH}$ and the parent material's Si/Al ratio. Alternatively, intraporous copper oxide nanoclusters can be formed during dehydration and calcination of over-exchanged copper zeolites via migration and sintering of $\mathrm{CuOH}^{+}$species (vide supra, section 2.3). In ZSM-5, copper clusters have been detected in the $5.5 \AA$ micropores by $\mathrm{N}_{2}$ sorption and magnetic susceptibility measurements. ${ }^{167}$

No experimental study has so far demonstrated a conclusive link between methane $\mathrm{C}-\mathrm{H}$ activation and large $(n>3) \mathrm{Cu}_{n} \mathrm{O}_{x}$ clusters in the zeolitic micropores. Tomkins et al. suggest highly dispersed, sub-nanometer copper oxide clusters as active sites based on TEM and EXAFS results ${ }^{155}$, and XPS confirms the reduction of a large 
fraction of the $\mathrm{Cu}^{2+}$ in the sample over 13 hours of methane reaction at $200^{\circ} \mathrm{C}$ at 6 bar. The same oxidized copper clusters may also be active in the catalytic methane to methanol conversion reported by Narsimhan et al. ${ }^{154}$ They observe absorption bands at 20800, 26800 and $30000 \mathrm{~cm}^{-1}$ during steady-state methanol production with in situ DRUV-Vis spectroscopy, which may be related to sub-nanoscale copper oxide species. DFT results indicate a positive effect of increased nuclearity on the activation of methane and support the stability of $\mathrm{Cu}_{n} \mathrm{O}_{x}, 2 \leq x \leq 5$ species in the $8 \mathrm{MR}$ channels of MOR and ZSM-5, with an increase in stability with increasing nuclearity. ${ }^{54}$ Verma et al. showed with DFT that $\mathrm{Cu}_{x} \mathrm{O}_{x}, \mathrm{x} \leq 8$ clusters can grow from nucleated dimers in the 8MR of $\mathrm{CHA} .{ }^{168}$ It should be noted that the aluminium atoms in this DFT model are placed on opposite sides of the 8MR. This is unlike the Al-Si-O-Si-O-Al chain in the 8MR, proposed to preferentially stabilize the dinuclear $\left[\mathrm{Cu}_{2} \mathrm{O}\right]^{2+}$ active site. ${ }^{37}$

4.5.3 - Comparative Reactivity of $\mathrm{Cu}_{n} \mathrm{O}_{x}$ Clusters. The mono- $\mu$-oxo dicopper sites and tricopper sites identified on Cu-MFI and Cu-MOR and discussed respectively in section 4.1 and section 4.5.1 require high temperature activation to form a reactive oxygen which abstracts a hydrogen atom from methane at $\mathrm{T} \leq 200^{\circ} \mathrm{C}$. At this reaction temperature, a strongly chemisorbed product is formed rendering the reaction stoichiometric. At reactivation temperature, the product is thermally decomposed to form $\mathrm{CO}_{2}$ and $\mathrm{H}_{2} \mathrm{O}$. Yields of methanol upon extraction and apparent activation energies of HAA are given in table 7 for various materials. Regardless of the existence of a stoichiometric regime, some copper exchanged zeolites perform methane activation under a catalytic regime at $\mathrm{T}>200^{\circ} \mathrm{C}$ in the presence of $\mathrm{CH}_{4}, \mathrm{O}_{2}$ and steam, although 
conversion and selectivity towards methanol remain very low. Active catalysts and their corresponding reaction rates are given in table 8 . The active sites in the catalytic regime are unlikely to be the same as those of the stoichiometric regime, and it has to be investigated whether the di- and trinuclear clusters are stable under reaction conditions above 473K.

Table 7. Yields of methanol and activation energies for the stoichiometric conversion.

$\begin{array}{llllll}\text { catalyst } & \text { Si/Al } & \text { Cu/Al } & \text { Yield }\left(\mu \mathrm{mol} \mathrm{g}^{-1}\right) & \mathrm{E}_{\mathrm{a}}(\mathrm{kcal} / \mathrm{mol}) & \text { Ref. } \\ \text { CuNaMFI } & 12 & 0.58 & 8.2^{E} ; 21.7^{B} & 15.8^{X} & 36,37 \\ \text { CuHMFI } & 14 & \leq 0.67 & \leq 89^{M} & 15.1^{T} & 67,79 \\ \text { CuNaMFI } & 13.6 & 0.37 & 37^{S} & / & 154 \\ \text { CuHMFI } & 13.2 & 0.31 & 82^{S} & / & 154 \\ \text { CuNaMOR } & 8.8 & 0.43 & 11.34^{E} & \text { MOR1: } 14.7 ; \text { MOR2: } 11.1^{X} & 36,63 \\ \text { CuHMOR } & 11 & \leq 0.40 & \leq 140^{M} & 17.7^{T} & 55,79\end{array}$

$E$ Yield as measured by methanol from liquid phase extraction; ${ }^{B}$ Yield as measured by oxidized carbon desorption upon thermal treatment; ${ }^{X}$ Experimental apparent activation enthalpy; ${ }^{M}$ Yield as measured by total activated methane; ${ }^{T}$ calculated activation energy; ${ }^{S}$ Yield as measured by methanol from steam extraction at $483 \mathrm{~K}, \mathrm{P}_{\mathrm{H}} \mathrm{O}=3.2 \mathrm{kPa}$.

Table 8. Rates of methanol formation in the catalytic regime

$\begin{array}{lllllll}\text { catalyst } & \text { Si/Al } & \text { Cu/Al } & \text { Temperature }(\mathrm{K}) & \text { Rate }\left(\mu \mathrm{mol} \mathrm{h}^{-1} \mathrm{~g}^{-1}\right)^{a} & \mathrm{E}_{\mathrm{a}}^{\mathrm{Ap}}(\mathrm{kcal} / \mathrm{mol}) & \begin{array}{l}\text { Ref. } \\ \text { CuNaMFI }\end{array} \\ 13.6 & 0.37 & 483 & 0.88 & 12.9 \pm 1.2 & 154 \\ \text { CuNaMFI } & 13.1 & 0.17 & 483 & 0.51 & 11.2 \pm 0.5 & 154 \\ \text { CuHMFI } & 14.1 & 0.38 & 483 & 1.51 & 19.1 \pm 0.5 & 154 \\ \text { CuHMFI } & 13.9 & 0.13 & 483 & 0.84 & 21 \pm 1.4 & 154 \\ \text { CuNaMOR } & 11.4 & 0.14 & 483 & 0.30 & 22 \pm 0.7 & 154 \\ \text { CuHMOR } & 11.1 & 0.14 & 483 & 0.84 & 35.6 \pm 0.5 & 154 \\ \text { CuHMFI } & 11.5 & 0.44 & 543 & 6 \pm 1 & / & 152 \\ \text { CuNaMOR } & 5 & 0.33 & 543 & 2 \pm 0.1 & / & 152 \\ \text { CuHMOR } & 5 & 0.30 & 543 & 10 \pm 3 & / & 152 \\ \text { CuHSSZ-13 } & 13.8 & 0.50 & 483 & 3.12 \pm 0.01 & 23.9 \pm 0.5 & 154 \\ \text { CuHSSZ-13 } & 12 & 0.41 & 543 & 28 \pm 1 & 34 \pm 7 & 152\end{array}$

${ }^{a}$ Rate is given in $\mu \mathrm{mol}$ methanol desorbed from the catalyst per hour per gram of catalyst at $483 \mathrm{~K}, \mathrm{P}_{\text {H2O }}=$ $3.2 \mathrm{kPa}$.

The most reliable apparent activation energies are those determined from the decrease of the LMCT band of the $\left[\mathrm{Cu}_{2} \mathrm{O}\right]^{2+}$ band around $22000 \mathrm{~cm}^{-1}$ identified on CuNaMOR and CuNaMFI (figure 35), for which the corresponding mechanism was discussed in section 
4.2. For the proposed trinuclear active site only a theoretical activation energy is calculated. The tricopper $\left[\mathrm{Cu}_{3}(\mu-\mathrm{O})_{3}\right]^{2+}$ active species is expected to hold a substantial radical character on the extra-framework oxygen ligands in the ground state, resembling the radical character found in the transition state of the mono- $\mu$-oxo dicopper site. It is proposed that a SOMO mainly consisting of the p-orbital of a bridging oxygen ligand cleaves the $\mathrm{C}-\mathrm{H}$ bond through interaction with the empty antibonding $\sigma^{*}(\mathrm{CH})$ orbital with an activation barrier of $17.7 \mathrm{kcal} / \mathrm{mol} .{ }^{55}$ The proposed orbital interaction is nucleophilic in nature, however the reaction results in reduction of a bridging oxyl to a hydroxide-level species. This is electrophilic chemistry, consistent with other metal/oxo active sites in zeolites ${ }^{35,37,134}$ and other late transition metals ${ }^{169}$. A similar activation energy of 17 $\mathrm{kcal} / \mathrm{mol}$ was calculated for the hydroxylated $\left[\mathrm{Cu}_{3}(\mu-\mathrm{OH})_{2}(\mu-\mathrm{O})\right]$ species. ${ }^{79}$ The methyl radical produced in HAA can either form an adsorbed methanol via a rebound ${ }^{170}$ of the hydroxyl, bind to another extra-framework oxygen, bind to a framework oxygen, or heterolytically bind to one of the coppers in the cluster. ${ }^{79}$ An equivalent rebound mechanism was proposed for the mono- $\mu$-oxo dicopper sites. ${ }^{37}$

The data in tables 7 and 8 reveal two trends. First, at comparable Si/Al and $\mathrm{Cu} / \mathrm{Al}$, the methanol yield of the $\mathrm{CuH}$-zeolites exceeds that of the CuNa-zeolites both in the stoichiometric and in the catalytic regime. Second, the apparent activation energies measured for the CuH-zeolites are higher than those for the CuNa-zeolites in the catalytic regime. Two reasons can be advanced to explain the difference in activation energy of $\mathrm{CuH}$-zeolites and CuNa-zeolites; the first is a difference in active site composition and structure; the second is a difference in the siting of the active site in the zeolite structure. In H-zeolites the protons are preferentially located in the most open 
parts of the zeolitic structures. ${ }^{81-83}$ This means that the di- or trinuclear $\mathrm{Cu}$ clusters are formed in the more confined parts of the zeolitic structures, where confinement effects more strongly influence the stability of reaction products and transition states. For MOR these are the $8 \mathrm{MR}$ side pockets. Analogous to observations on the mono- $\mu$-oxo dicopper in Cu-MOR, a 50\% lower methanol yield obtained per copper from CuHZSM-5 hosted $\left[\mathrm{Cu}_{3}(\mu-\mathrm{O})_{3}\right]^{2+}$ active sites compared to that from CuHMOR hosted $\left[\mathrm{Cu}_{3}(\mu-\mathrm{O})_{3}\right]^{2+}$ was hypothesized to be related to confinement effects. ${ }^{67}$ In mordenite, the preferential substitution of $\mathrm{Al}^{3+}$ in the $8 \mathrm{MR}$ side-pocket pore-mouth would limit the speciation of $\left[\mathrm{Cu}_{3}(\mu-\mathrm{O})_{3}\right]^{2+}$ to these specific locations, where confinement effects enable their reactivity and selectivity. In ZSM-5, aluminium pairs can be situated in various zeolite positions, only half of which would sustain $\left[\mathrm{Cu}_{3}(\mu-\mathrm{O})_{3}\right]^{2+}$ in a suitable environment to enable its reactivity towards methane activation at $200^{\circ} \mathrm{C}$ and 1 atm. ${ }^{67}$ Periodic DFT calculations on $\left[\mathrm{Cu}_{2}(\mu-\mathrm{O})\right]^{2+}$ sites in MFI and small-pore zeolites $\mathrm{CHA}, \mathrm{AEI}$ and $\mathrm{AFX}$ further indicate the influence of zeolite confinement on the site geometry and on the activation barrier for methane $\mathrm{C}-\mathrm{H}$ cleavage. ${ }^{171}$

Expansion of the copper oxide cluster model to $\left[\mathrm{Cu}_{4} \mathrm{O}_{4}\right]^{2+}$ and $\left[\mathrm{Cu}_{5} \mathrm{O}_{5}\right]^{2+}$ demonstrates a further stabilization of the bound $\mathrm{CH}_{3}$ and $\mathrm{OH}$ fragments resulting from methane HAA with increasing cluster size. It is suggested that larger clusters, with more linkages between copper atoms and extra-framework oxygen ligands can be expected to be more resistant to disturbances caused by the stabilization of reaction intermediates ${ }^{54}$. This does not, however, take into account the energy of the transition state in activating the $\mathrm{C}-\mathrm{H}$ bond, and therefore cannot be seen as a direct indication for improved active site reactivity. It is possible that reactivity of such sites is enhanced by 
under-coordinated oxygen species

chemisorbed to the cluster upon high

temperature oxygen treatment. Such Figure 45. left: The high energy $\mathrm{CuO}(010)$ surface of bulk $\mathrm{CuO}$ with two-coordinated surface oxygen oxygen species attached to the high atoms, right: DFT model of $\mathrm{CH}_{4}$ activation over the $\mathrm{CuO}(010)$ surface. Reproduced from reference 172 energy $\mathrm{CuO}(010)$ surface of bulk copper with permission. Copyright 2016, Royal Society of Chemistry.

oxide were calculated by Varghese et al. to cleave the methane $\mathrm{C}-\mathrm{H}$ bond with a barrier of only $14.4 \mathrm{kcal} / \mathrm{mol}$ in a DFT simulation (figure 45 ). ${ }^{172}$ In this case, high surface area copper oxide particles, preferably with a large availability of the most active surface face, would be the only requisite regardless of the support material. The zeolite matrix then does not contribute to the intrinsic reactivity of the active site, and its role would be limited to the stabilization of the most active copper phase. All considerations about the reactivity of these copper clusters remain, however, purely computational, and experimental corroboration is needed.

4.5.4 - Mononuclear $[\mathrm{Cu}(\mathrm{OH})]^{+}$Active Sites. Mononuclear copper sites in zeolites are usually considered spectator species in partial methane oxidation, or their role is limited to a co-catalyst effect. ${ }^{173}$ With the observation of undefined, water stable active sites in various microporous materials in mind $84,152,154,173$, researchers should, however, keep the option open. $[\mathrm{CuOH}]^{+}$extra-framework cations have been experimentally confirmed to be present in $\mathrm{Cu}-\mathrm{CHA}$ upon high temperature activation in an $\mathrm{O}_{2}$ atmosphere by the observation of the $\mathrm{v}(\mathrm{OH})$ stretch vibration of $[\mathrm{CuOH}]^{+}$at $3650 \mathrm{~cm}^{-1}$ in FTIR ${ }^{72,76}$ and have been proposed as active sites in low temperature SCR. ${ }^{70}$ The location of $[\mathrm{CuOH}]^{+}$ in the $\mathrm{CHA} 8 \mathrm{MR}$ with a single $\mathrm{Al}^{3+}$ substitution was proposed by a combination of 
experimental (XAS, FTIR, chemical titration) and theoretical DFT work. ${ }^{71} \mathrm{~A}[\mathrm{CuOH}]^{+}$site has been calculated to be stable in the 8MR of the CHA topology in the presence of moisture, and is predicted to exhibit a reactivity towards methane HAA comparable to the experimental Cu-SSZ-13 catalyst ${ }^{154}$ by quantum chemical calculations ${ }^{74}\left(E_{a}=26.3\right.$ $\mathrm{kcal} / \mathrm{mol}$ by DFT vs $\mathrm{E}^{\mathrm{ap}}=23.9 \mathrm{kcal} / \mathrm{mol}$, table 7). Likewise, ligated $[\mathrm{CuO}]^{+}$and $[\mathrm{CuOH}]^{+}$ sites for methane $\mathrm{C}-\mathrm{H}$ activation have been identified outside of zeolite catalysis ${ }^{174-176}$, and their reactivity and electronic properties have been evaluated with theoretical postHartree-Fock and DFT methods. ${ }^{177}$ Nevertheless, a conclusive experimental link between $[\mathrm{CuOH}]^{+}$and methane activation is still lacking. 


\section{5 - Fe/O Active Sites in Zeolites.}

5.1 - Introduction. The most widely studied Fe-zeolite, $\mathrm{N}_{2} \mathrm{O}$-activated Fe-ZSM-5, is notable for oxidizing methane to methanol rapidly and selectively at room temperature and pressure. ${ }^{33,178-180} \mathrm{Fe}$-zeolites also catalyze the low temperature hydroxylation of benzene to form phenol, ${ }^{33,94,181,182}$ and for this reaction, $\mathrm{Li}$ and coworkers have identified a spectroscopic intermediate assigned as a ferric-phenolate species. ${ }^{94}$ Selective hydroxylation of methane and benzene occurs at an extra-lattice active site called $\alpha$ $\mathrm{Fe}(\mathrm{II})$, which is activated by nitrous oxide to form the reactive intermediate $\alpha-0 .{ }^{181,183}$ Analogous active sites are stabilized in a number of other lattice topologies. ${ }^{58,181,182}$ The geometric and electronic structures of these sites, and factors contributing to their reactivity were defined recently using VTVH-MCD spectroscopy (see section 3.3) coupled to experimentally-validated DFT models - the first application of MCD to distinguishing an active site in a heterogeneous system. ${ }^{35}$ These studies showed the exceptional reactivity of $\alpha-O$ derives from a constrained coordination geometry enforced by the rigid zeolite lattice (see 5.3.1, 5.3.2), producing what is referred to in bioinorganic chemistry as an 'entatic' state (see 6.3.3). ${ }^{35,118}$ It has also been shown that Fe-ZSM-5 converts methane to methanol using aqueous peroxide at low temperature (see 5.4.1). ${ }^{92}$ Here, reactivity occurs at active site(s) that are distinct from $\alpha-\mathrm{Fe}(\mathrm{II}) / \alpha-\mathrm{O}$, but the nature of the active Fe component in this system is unclear. ${ }^{93,184}$ 


\section{2 - The $\alpha-\mathrm{Fe}$ (II) Active Site}

5.2.1 $-\alpha-\mathrm{Fe}$ (II) Structure. Combined spectroscopic and computational studies of Fezeolites have defined $\alpha-\mathrm{Fe}(\mathrm{II})$ to be a mononuclear, high-spin $\mathrm{Fe}(\mathrm{II})$ center with square planar geometry (figure 46). ${ }^{35}$ This extra-lattice site binds in beta-type 6-memebered rings $\left(\beta-6 \mathrm{MR}\right.$ - figure 46 left), ${ }^{35}$ which are present in a number of lattice topologies (notably MFI, *BEA, and FER). ${ }^{40,185,186} \alpha-\mathrm{Fe}(\mathrm{II})$ binds to the $\beta$-6MR through two Al Tsites, each of which binds in a bidentate fashion. Thus the Fe(II) center is bound to four AIOsi ligands, each carrying a partial negative charge. Other configurations of AI T sites within the active site fail to reproduce the spectroscopically-defined spin Hamiltonian parameters of $\alpha-\mathrm{Fe}(\mathrm{II})$ (see 5.2.2). Currently, no structural data exist for $\alpha-\mathrm{Fe}(\mathrm{II})$, although spectroscopically-calibrated DFT models predict Fe-O bond lengths of 1.99$2.01 \AA$ for $\alpha-\mathrm{Fe}(\mathrm{II})$ in $\mathrm{Fe}(\mathrm{II})-\mathrm{BEA}{ }^{35}$ In most $\mathrm{Fe}(\mathrm{II})$-zeolites, one face of $\alpha$ - $\mathrm{Fe}(\mathrm{II})$ is sterically unencumbered, while the other is sterically protected. ${ }^{185}$ (An exception is FER,
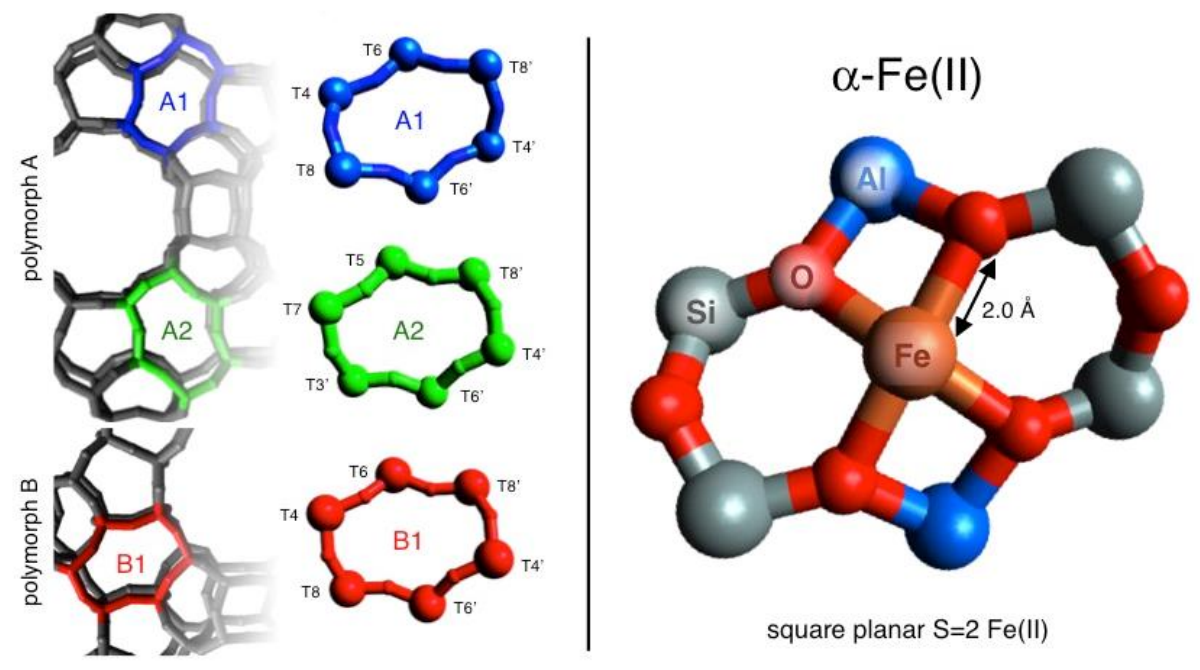

Figure 46. $\beta-6 \mathrm{MR}$ motifs in BEA, with T-site labels (left). The predicted structure of $\alpha-\mathrm{Fe}(\mathrm{II})$ sited in ring A1 is shown to the right, with Al T-sites at positions T6 and T6'. Adapted from reference ${ }^{35}$. 
whose $\beta-6 \mathrm{MRs}$ are accessible from both faces). ${ }^{187} \alpha-\mathrm{Fe}(\mathrm{II})$ therefore has a readilyaccessible open coordination site that is required for function: accepting an $\mathrm{O}$ atom from $\mathrm{N}_{2} \mathrm{O}$ to form $\alpha-\mathrm{O}$, the reactive intermediate in selective hydrocarbon oxidation (vide infra).

Based on variations in $\beta-6 \mathrm{MR}$ structure, some structural perturbations are expected for $\alpha-\mathrm{Fe}(\mathrm{II})$ across different zeolite lattices. The crystal structure of BEA shows three species of $\beta-6 \mathrm{MR}$ (figure 46 , left). ${ }^{186}$ Ring $A 1$ and $A 2$ are rigorously $C_{2-}$ symmetric, while ring $\mathrm{B} 1$ is slightly perturbed from ideal $\mathrm{C}_{2}$ symmetry. The $\mathrm{T}$-sites that bind Fe are separated by 5.1-5.6 $\AA$, and the plane defined by the bidentate bite of these T-sites is angled out of the plane defined by the ring. Spectroscopy coupled to DFT shows this leads to rhombic distortions of the active site, resulting in two inequivalent pairs of (trans) Fe-O bonds, and an out-of-plane displacement of the $\mathrm{Fe}(\mathrm{II})$ center. $^{35}$ In the MFI lattice (ZSM-5), the $\beta$-6MRs are C1-symmetric, and also show significant distortions from planarity. ${ }^{188}$ In contrast to the BEA and MFI topologies, a single species

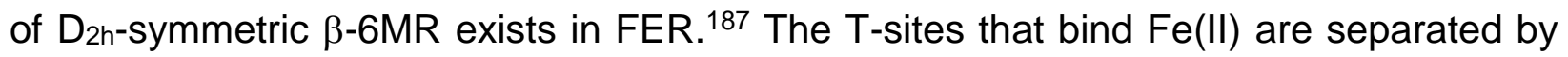
$5.9 \AA$, and the plane defined by the bidentate bite of these T-sites lies in the plane defined by the ring. The face-to-face separation of neighboring $\beta-6 M R s$ in FER is also relatively short (7.5 $\AA$ versus $>9 \AA$ in other lattices), and it has been proposed that this allows neighboring $\alpha$ - $\mathrm{Fe}(\mathrm{II})$ sites in FER to act cooperatively in the activation of $\mathrm{N}_{2} \mathrm{O} \cdot{ }^{58,189}$ For FER and $\mathrm{MFI}$, it is not yet understood how these differences in lattice geometry perturb the geometric/electronic structure and reactivity $\alpha-\mathrm{Fe}(\mathrm{II})$. 
5.2.2 - $\alpha-\mathrm{Fe}$ (II) Spectroscopy. Several spectroscopic methods have been used to focus on the reduced $\alpha-F e(I I)$ active site and define its geometric and electronic structure. ${ }^{35}$ These studies have defined unique spectral features that reflect the unusual combination of square planar geometry and high spin $(S=2)$ ferrous electronic
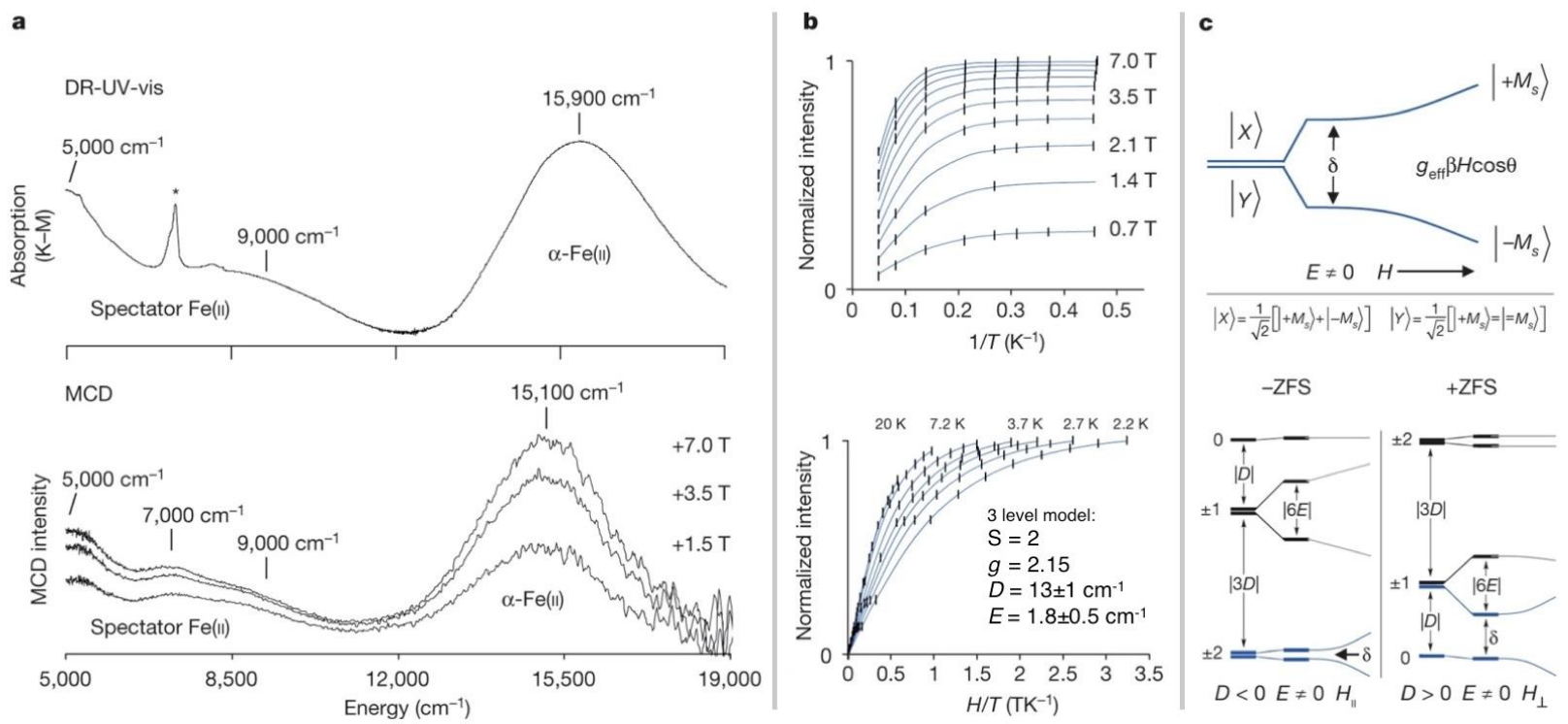

Figure 47. a) Comparison of $298 \mathrm{~K}$ DR-UV-vis data $\left({ }^{*}=\mathrm{OH}\right.$ overtone) and variable-field $3 \mathrm{~K}$ MCD data from $\mathrm{Fe}(\mathrm{II})-\mathrm{BEA}(\mathrm{Si} / \mathrm{Al}=12,0.3 \mathrm{wt} \% \mathrm{Fe})$. b) Top, Saturation magnetization isofields for the $15,100 \mathrm{~cm}^{-1}$ band of $\alpha-\mathrm{Fe}(\mathrm{II})(1 \sigma$ error bars are in black; the fit is in blue). Bottom, Saturation magnetization isotherms for $\alpha-F e(I I)$ (fit in blue), including best-fit spin Hamiltonian parameters. c) Top, effects of rhombic ZFS $(\delta)$ and external magnetic field on a non-Kramers doublet. Bottom, comparison of + ZFS and $-\mathrm{ZFS} S=2$ spin manifolds, including effects due to axial $(D \neq 0)$ and rhombic $(E \neq 0) Z F S$. Levels identified with the nonKramers doublet model shown in the top of the figure are highlighted in blue. Adapted with permission from reference ${ }^{35}$. Copyright 2016 Nature Publishing Group

structure. ${ }^{35,190-195} \mathrm{Fe}(\mathrm{II})$-BEA contains the most extensively characterized $\alpha-\mathrm{Fe}(\mathrm{II})$ site, while limited data exist for $\alpha-\mathrm{Fe}(\mathrm{II})$ in other zeolite lattices. In DR-UV-vis (figure $47 a$, top), $\alpha-\mathrm{Fe}(\mathrm{II})$ in $\mathrm{Fe}(\mathrm{II})-\mathrm{BEA}$ exhibits a single, high energy ligand field band at $15,900 \mathrm{~cm}^{-}$

1. As shown in figure 48, a similar feature is observed in Fe-ZSM-5 and Fe-FER but not Fe-MOR. ${ }^{35}$ Based on Abs spectroscopy of structurally-defined high spin $\mathrm{Fe}(\mathrm{II})$ sites coupled to electronic structure calculations, the red band of $\alpha-\mathrm{Fe}(\mathrm{II})$ was assigned as 
the $3 d_{z 2} \rightarrow 3 d_{x 2-y z}$ ligand field transition of a square planar site. ${ }^{35,190,193,195}$ The high energy of this transition reflects the axial/equatorial anisotropy of the ligand field provided by the $\beta-6 \mathrm{MR}$, as well as the unique stability of $3 d_{z 2}$ in square planar geometry brought about by $3 d_{z 2} / 4 s$ mixing in the absence of axial ligands. DR-UV-vis bands at lower energy $\left(<12,000 \mathrm{~cm}^{-1}\right)$ are also present in $\mathrm{Fe}(\mathrm{II})$-zeolites, but these are assigned as ligand field bands of spectator Fe(II) sites. ${ }^{35}$

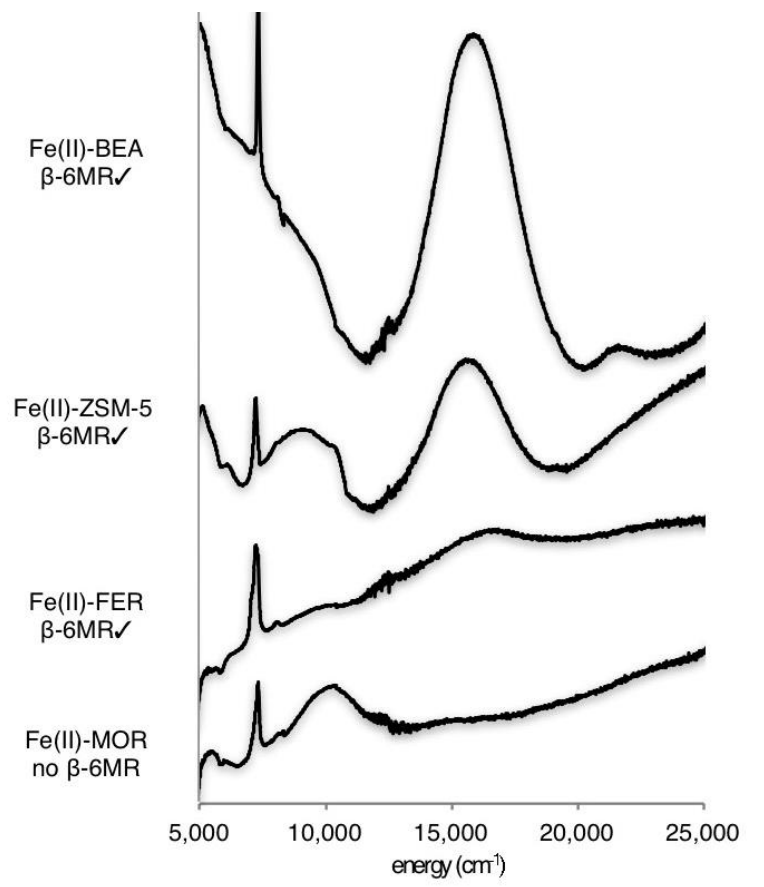

Figure 48. Ligand field spectra of three zeolites known to stabilize $\alpha-\mathrm{Fe}$ (II) (BEA, ZSM-5, FER) as well as $\mathrm{Fe}(\mathrm{II})-\mathrm{MOR}$, which does not contain $\alpha$ $\mathrm{Fe}(\mathrm{II})$. Adapted with permission from reference ${ }^{35}$. Copyright 2016 Nature Publishing Group
In low-temperature MCD (figure 47a, bottom), the $3 d_{z 2} \rightarrow 3 d_{x 2-y z}$ transition of $\alpha-$ $\mathrm{Fe}(\mathrm{II})$ in $\mathrm{Fe}(\mathrm{II})-\mathrm{BEA}$ appears as a positive band at $15,100 \mathrm{~cm}^{-1} .35$ This feature is sensitive to both temperature and field due to the paramagnetic ground state of $\alpha$ $\mathrm{Fe}(\mathrm{II})$. It gains intensity through what is formally a temperature-dependent $B$-term mechanism (see 3.3.2), as is evident from VTVH-MCD isofields from the $3 d_{z 2} \rightarrow 3 d_{x 2-y 2}$ transition (figure 47b, top), which show field dependence in the low-temperature

saturation limit. This reflects changes to the ground state wavefunction brought about by field-induced mixing of Zeeman sublevels - a characteristic behavior of non-Kramers (integer spin) systems. ${ }^{113,116}$ At zero field, a non-Kramers doublet ground state is split by the amount $\delta$ due to rhombic ZFS (figure 47c, top). The $\pm \mathrm{M}_{\mathrm{s}}$ wavefunctions also mix equally, forming linear combinations $|\mathrm{X}\rangle$ and $|\mathrm{Y}\rangle$. The levels undergo an additional 
Zeeman splitting in the presence of a magnetic field, and the pure $\left|-M_{s}\right\rangle$ and $\left|+M_{s}\right\rangle$ states are recovered in high fields (figure $47 \mathrm{c}$, top). A $g_{\text {eff }}=8.6$ indicates $\alpha$-Fe(II) is an $\mathrm{S}=2$ system, and a large $\delta=9.0 \mathrm{~cm}^{-1}$ indicates this is a positive zero-field split system (see figure $47 \mathrm{c}$, bottom). VTVH-MCD isotherms from the $15,100 \mathrm{~cm}^{-1}$ transition (figure 47b, bottom) are highly nested due to a large and positively-signed axial zero-field splitting $\left(D=13 \pm 1 \mathrm{~cm}^{-1}\right)$ of the $\mathrm{S}=2$ ground state of $\alpha-\mathrm{Fe}(\mathrm{II}) \cdot{ }^{35}$ This large positive $D-\mathrm{a}$ characteristic of high-spin square planar $\mathrm{Fe}(\mathrm{II})$ - is a consequence of spin-orbit coupling between the $d_{z 2}$ ground state and low-lying $d_{x z} / d_{y z}$ ligand field excited states. VTVHMCD data also indicate a rhombic site distortion $(E / D=0.14)$ of $\alpha-F e(I I)$ due to deviations from ideal square-planar

geometry (see 6.2.1).

In room-temperature Mössbauer spectroscopy (figure 49), $\alpha-\mathrm{Fe}(\mathrm{II})$ in $\mathrm{Fe}(\mathrm{II})-\mathrm{BEA}$ exhibits a single quadrupole-split doublet with an isomer shift of ca. $\delta=0.89 \mathrm{~mm} / \mathrm{s}$ and quadrupole splitting $\left|\Delta E_{\mathrm{Q}}\right|=0.55 .{ }^{35}$ Similar features have been reported for $\mathrm{Fe}(\mathrm{II})-\mathrm{FER}\left(\delta=0.94 \mathrm{~mm} / \mathrm{s},\left|\Delta E_{\mathrm{Q}}\right|=0.69\right.$ $\mathrm{mm} / \mathrm{s})$ and $\mathrm{Fe}(\mathrm{II})-\mathrm{ZSM}-5\left(\delta=0.89 \mathrm{~mm} / \mathrm{s},\left|\Delta E_{\mathrm{Q}}\right|=0.74\right.$ $\mathrm{mm} / \mathrm{s}$ ), but at the time these were not assigned to $\alpha$ $\mathrm{Fe}(\mathrm{II}){ }^{180}$ These are unusually small quadrupole splittings for $S=2$ ferrous sites, where $\left|\Delta E_{Q}\right|$ typically

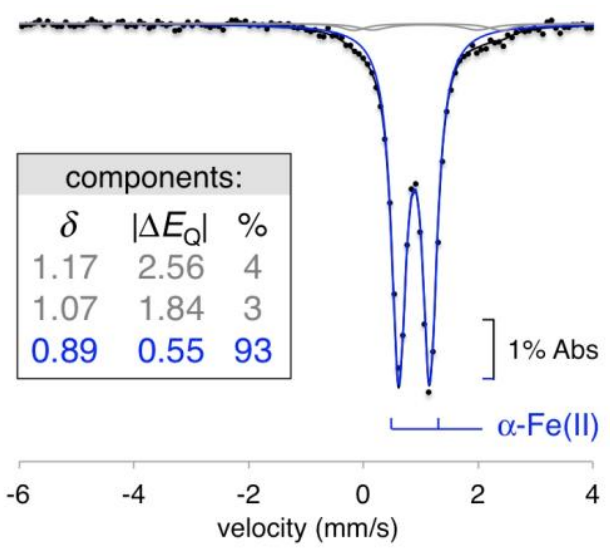

Figure 49. $300 \quad \mathrm{~K}$ Mössbauer spectrum of $\mathrm{Fe}(\mathrm{II})-\mathrm{BEA}(\mathrm{Si} / \mathrm{Al}=12,0.3$ wt\% $\mathrm{Fe}$ ), with the $\alpha-\mathrm{Fe}(\mathrm{II})$ doublet shown in blue. Adapted with permission from reference 35 . Copyright 2016 Nature Publishing Group

exceeds $1.5 \mathrm{~mm} / \mathrm{s}$. For $\mathrm{S}=2$ square planar $\mathrm{Fe}(\mathrm{II})$ sites, however, an equatorial distribution of ligand electron density together with an axial distribution of $d$ electron 
density due to a doubly-occupied $3 \mathrm{~d}_{z 2}$ orbital (see section 3.1.2) leads to nearcancellation of large, oppositely signed lattice and valence contributions (respectively) to $\Delta E_{\mathrm{Q} .}{ }^{194}$

5.2.3 - Reactivity of $\alpha-\mathrm{Fe}$ (II). Studies of the reactivity of $\alpha-\mathrm{Fe}(\mathrm{II})$ are currently limited to its role in O-atom transfer from $\mathrm{N}_{2} \mathrm{O} .^{58,189}$ In this reaction, $\alpha-\mathrm{Fe}(\mathrm{II})$ donates two electrons to cleave the $\mathrm{N}-\mathrm{O}$ bond of $\mathrm{N}_{2} \mathrm{O}$, releasing $\mathrm{N}_{2}$ and forming $\alpha-\mathrm{O}$. This occurs rapidly at moderate temperatures (e.g. $200 \mathrm{C}$ ), with an apparent activation enthalpy of $10 \mathrm{kcal} / \mathrm{mol}$ in Fe-ZSM-5. ${ }^{32}$ Similar reactivity is observed for $\mathrm{Fe}(\mathrm{II})-\mathrm{BEA} .{ }^{58,189} \mathrm{Fe}(\mathrm{II})$-FER has improved reactivity relative to other $\mathrm{Fe}(\mathrm{II})$-zeolites (vide infra). ${ }^{58,189}$ Molecular-level insight into the activation of $\mathrm{N}_{2} \mathrm{O}$ by $\alpha-\mathrm{Fe}(\mathrm{II})$ is lacking, however two general factors enabling rapid $\mathrm{N}_{2} \mathrm{O}$ reduction have been defined in studies of Cu-zeolites (Cu-ZSM-5) and the $\mathrm{Cu}_{z}$ cluster of $\mathrm{N}_{2} \mathrm{OR} \cdot{ }^{135,151}$ First, $\mathrm{N}_{2} \mathrm{O}$ must bind to the active site with a geometry that facilitates the transfer of two electrons required to cleave the $\mathrm{N}-\mathrm{O}$ bond. (Unlike the multinuclear $\mathrm{Cu}$ active sites of $\mathrm{N}_{2} \mathrm{O}$ reduction, however, the mononuclear $\alpha$ $\mathrm{Fe}$ (II) active site can reduce $\mathrm{N}_{2} \mathrm{O}$ by two electrons without the participation of a second metal center.) Second, the formation of a strong metal-oxo/hydroxo bond in the oxidized product creates a large driving force for $\mathrm{O}$ atom transfer.

The thermodynamics of $\mathrm{N}_{2} \mathrm{O}$ reduction have been explored computationally for $\alpha$ $\mathrm{Fe}(\mathrm{II})$ sites in $\mathrm{Fe}(\mathrm{II})-\mathrm{FER} .{ }^{189} \mathrm{~N}_{2} \mathrm{O}$ can bind to $\alpha$-Fe(II) terminally by either the $\mathrm{O}$ or $\mathrm{N}$ atom, forming a square pyramidal intermediate. The $\mathrm{N}$-bound sites are predicted to be more stable $(8-12 \mathrm{kcal} / \mathrm{mol}$ binding energy versus $6-9 \mathrm{kcal} / \mathrm{mol}$ for $\mathrm{O}$ binding), but are unproductive in $\mathrm{O}$ atom transfer. The $\mathrm{O}$-bound intermediates feature axial $\mathrm{Fe}-\mathrm{O}$ bond 
lengths of 2.16-2.26 $\AA$, bent Fe-O-N angles (ca. $120^{\circ}-130^{\circ}$ ), and minimal changes to O$\mathrm{N}$ and $\mathrm{N}-\mathrm{N}$ bond lengths. The predicted electronic structure of this intermediate was not discussed. The transition state for O-atom transfer lies $18-19 \mathrm{kcal} / \mathrm{mol}$ above the ground state, translating to an apparent activation enthalpy of $9-13 \mathrm{kcal} / \mathrm{mol}$. This agrees well with the value from $\mathrm{Fe}(\mathrm{II})-\mathrm{ZSM}-5,{ }^{32}$ however no experimental kinetic parameters exist for $\mathrm{N}_{2} \mathrm{O}$ activation by $\mathrm{Fe}(\mathrm{II})-\mathrm{FER}$. Electronic structure contributions to $\mathrm{N}-\mathrm{O}$ cleavage also have not yet been defined, and the strength of the product iron-oxo bond has not been evaluated (however see section 5.3). The geometry of the FER lattice is unique in that it may allow neighboring $\alpha-\mathrm{Fe}(\mathrm{II})$ sites to act cooperatively in $\mathrm{N}_{2} \mathrm{O}$ reduction. This has been proposed as an explanation for the $\sim 3$-fold enhancement of rate in $\mathrm{N}_{2} \mathrm{O}$ reduction for $\mathrm{Fe}(\mathrm{II})-\mathrm{FER}$ relative to $\mathrm{Fe}-\mathrm{BEA}$ and Fe-ZSM-5 (at $230 \mathrm{C}$ ). ${ }^{58,189}$ In this scheme, $\mathrm{N}_{2} \mathrm{O}$ forms a $\mu-1,3-\mathrm{O}, \mathrm{N}$ bridge between adjacent $\alpha-\mathrm{Fe}(\mathrm{II})$ centers. The apparent barrier for O-atom transfer calculated for this bridged intermediate is $3-4$ $\mathrm{kcal} / \mathrm{mol}$ (versus $9-13 \mathrm{kcal} / \mathrm{mol}$ for the mononuclear site). No explanation is provided for how $\mathrm{N}_{2} \mathrm{O}$ bridging tunes down the barrier, however, and no experimental kinetics data exist for comparison.

While the $\mathrm{N}_{2} \mathrm{O}$ reactivity of Fe-zeolites has been studied extensively, it is currently accepted that $\mathrm{O}_{2}$ does not function in $\mathrm{O}$ atom transfer to $\alpha-\mathrm{Fe}(\mathrm{II}) \cdot{ }^{32,183}\left(\mathrm{O}_{2}\right.$ may react with sites distinct from $\alpha-\mathrm{Fe}(\mathrm{II})$, however. $)^{95}$ This is in contrast to the Cu-zeolites, where $\mathrm{O}_{2}$ does transfer an $\mathrm{O}$ atom to the $2 \mathrm{Cu}(\mathrm{I})$ active sites of $\mathrm{Cu}-\mathrm{ZSM}-5$ and $\mathrm{Cu}-\mathrm{MOR}$ (see section 4.3). ${ }^{37,134,136} \mathrm{~A}$ key intermediate step to $\mathrm{O}$ atom transfer in the Cu-zeolites is reduction of $\mathrm{O}_{2}$ by two electrons to form a peroxy-level $\left[\mathrm{Cu}_{2}\left(\mathrm{O}_{2}\right)\right]^{2+}$ intermediate. ${ }^{136} \mathrm{In}$ this case, the $\mathrm{Cu}(\mathrm{l})$ centers are oxidized by one electron, meaning two electrons can be 
readily transferred to $\mathrm{O}_{2}$ without the formation of a strong metal-oxo bond. (This is in contrast to the reduction of $\mathrm{N}_{2} \mathrm{O}$, where $\mathrm{Fe}(\mathrm{IV})$-oxo or $\mathrm{Cu}(\mathrm{II})_{2}$-oxo bond formation drives electron transfer). The ability of $\alpha-\mathrm{Fe}(\mathrm{II})$ to transfer two electrons to $\mathrm{O}_{2}$ has not yet been evaluated. Many mononuclear non-heme $\mathrm{Fe}(\mathrm{II})$ centers that bind $\mathrm{O}_{2}$ exist in biology and model chemistry, but in the absence of exogenous electrons these generally activate $\mathrm{O}_{2}$ at the superoxy level, and do not go on to form stable peroxo-Fe(IV) species. Whether $\alpha-\mathrm{Fe}(\mathrm{II})$ fits with this trend remains to be experimentally evaluated.

\section{3 - The $\alpha$-O Reactive Intermediate}

5.3.1 - $\alpha-0$ Structure. Combined spectroscopic and computational studies of $\mathrm{N}_{2} \mathrm{O}$-activated Fe-zeolties have defined $\alpha-O$ to be a mononuclear, high-spin $(S=2)$

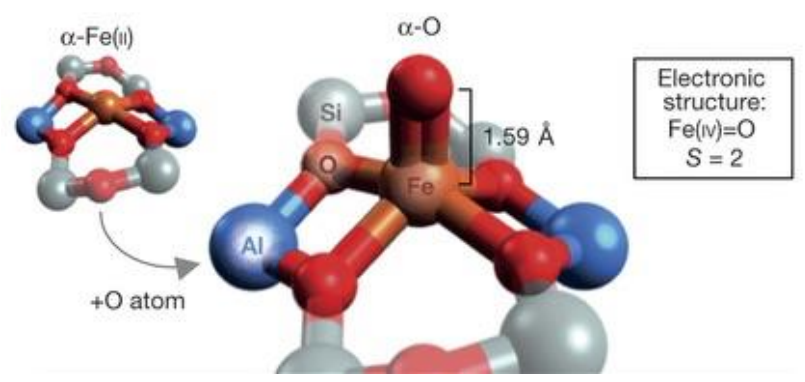
$\mathrm{Fe}(\mathrm{IV})=\mathrm{O}$ species $($ figure 50$) .{ }^{35} \mathrm{In}$ this square pyramidal site, equatorial ligation

Figure 50. DFT-predicted structure of $\alpha-\mathrm{O}$ in FeBEA. Adapted with permission from reference ${ }^{35}$. Copyright 2016 Nature Publishing Group

is provided by the $\beta-6 \mathrm{MR}$. The oxo ligand

occupies the axial position of the exposed face of the active site, and is thus readily accessible to substrates as large as benzene. Spectroscopically-calibrated DFT models of $\alpha-\mathrm{O}$ in Fe-BEA predict equatorial Fe-Olattice bond lengths of $2.00-2.02 \AA$ - highly similar to the $\mathrm{Fe}-\mathrm{O}_{\text {lattice }}$ bonds of $\alpha-\mathrm{Fe}(\mathrm{II})$. The $\mathrm{Fe}=\mathrm{O}$ bond is predicted to be short (1.59 $\AA$ ) and highly covalent due to the absence of a trans axial ligand. A similar geometric structure has been predicted for $\alpha-\mathrm{O}$ in Fe-ZSM-5. ${ }^{178}$ Currently, no structural data exist for $\alpha-O$ in any zeolite lattice, however. 
$\alpha-\mathrm{O}$ is the first $\mathrm{S}=2 \mathrm{Fe}(\mathrm{IV})=\mathrm{O}$ site with axial square pyramidal geometry to be identified. This geometry is inherently unstable for a high spin $\mathrm{d}^{4}$ site with a single strongly-bound ligand (oxo) and four weak ligands (lattice O). ${ }^{196,197}$ As shown in figure 51, shifting an equatorial lattice $O$ ligand to the vacant trans axial position would exchange a destabilizing equatorial $\sigma$-antibonding interaction (occupied $\sigma^{*} d_{x^{2}-y^{2}}$ ) for a stabilizing axial $\sigma$-bonding interaction (vacant $\sigma^{*} d_{z^{2}}$ ), resulting in stronger bonding between the $\mathrm{Fe}(\mathrm{IV})$ and the zeolite lattice. The strong ligand therefore prefers to bind in the equatorial plane. This creates a driving force for geometric distortion (ca. $6 \mathrm{kcal} / \mathrm{mol}$ by DFT) toward square pyramidal geometry with the oxo ligand in the equatorial plane. ${ }^{35}$ The role of the zeolite lattice is therefore twofold. First, the weak equatorial ligand field provided by the zeolite lattice stabilizes the $\mathrm{S}=2 \mathrm{Fe}(\mathrm{IV})=0$ electronic structure of $\alpha-O$. The rigid $\beta$ $6 \mathrm{MR}$ then opposes the distorting force associated with this electronic structure, enforcing the vacant trans axial position that is key to function. This defines an (right).

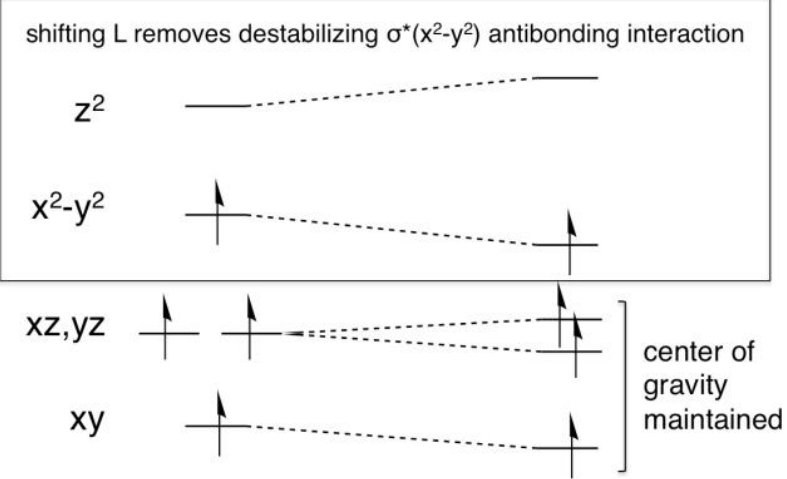

Figure 51. Walsh diagram illustrating the ligand field origin of the distorting force experienced by an axial square pyramidal $\mathrm{S}=2 \mathrm{Fe}(\mathrm{IV})=\mathrm{O}$ site (left) toward an equatorial square pyramidal structure

entatic state for $\alpha-O$, whereby rigid lattice constraints maintain an electronic structure that is associated with high reactivity (see 6.3.3 for detail). 
5.3.2 $-\alpha-O$ Spectroscopy. $\alpha-O$ has been studied in detail by DR-UV-vis, MCD, VTVHMCD, and Mossbauer spectroscopy. ${ }^{35}$ Fe(II)-BEA contains the most extensively characterized $\alpha-\mathrm{O}$ site, but some data also exist on $\alpha$-O in Fe-ZSM-5 (including DR-UV-vis, and resonance Raman). ${ }^{94}$ These studies have defined unique spectral features that reflect the $(S=2)$ ferryl oxo electronic structure of $\alpha-O$. In

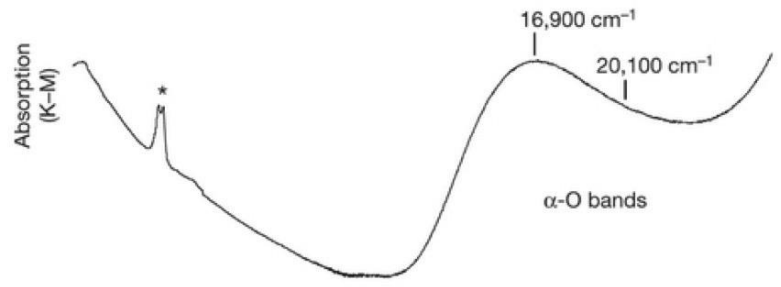
DR-UV-vis (figure 52, top), $\alpha-O$ in Fe-BEA exhibits three absorption features below $22,000 \mathrm{~cm}^{-1}$. The shoulder of a weak, low energy feature is seen between $5000-9000$ $\mathrm{cm}^{-1}$. At higher energy, there is a more intense $16,900 \mathrm{~cm}^{-1}$ band with a high energy shoulder at $\sim 20,000 \mathrm{~cm}^{-1}$. A similar absorption band is seen in $\mathrm{N}_{2} \mathrm{O}$ -
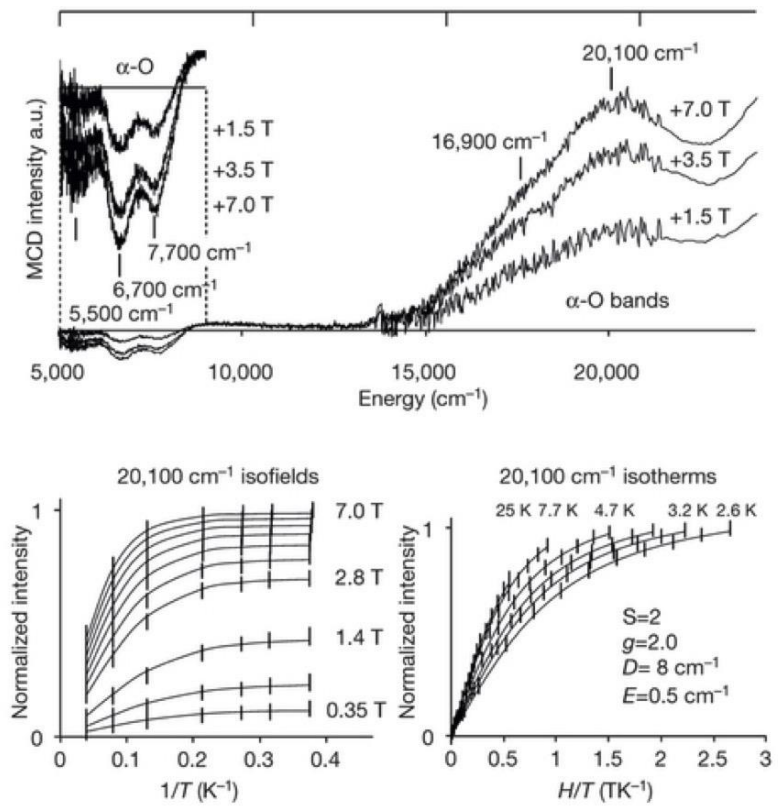

Figure 52. Comparison of $298 \mathrm{~K}$ DR-UV-vis data (top, ${ }^{*}=\mathrm{OH}$ overtone) and variable-field $3 \mathrm{~K} \mathrm{MCD}$ data (middle) from $\mathrm{N}_{2} \mathrm{O}$-activated $\mathrm{Fe}-\mathrm{BEA}(\mathrm{Si} / \mathrm{Al}=$ $12,0.3 \mathrm{wt} \% \mathrm{Fe}$ ), along with saturation magnetization isofields for the $20,100 \mathrm{~cm}^{-1}$ band of $\alpha-O$ (bottom left, $1 \sigma$ error bars are in black; the fit is in blue) and saturation magnetization isotherms for $\alpha-\mathrm{Fe}$ (II) (bottom right, fit in blue) with best-fit spin Hamiltonian parameters. Adapted with permission from reference ${ }^{35}$. Copyright 2016 Nature Publishing Group activated Fe-ZSM-5 at $16,500 \mathrm{~cm}^{-1}$, but this feature has not yet been correlated to $\alpha-\mathrm{O}$ through methane reactivity. ${ }^{94}$ In Raman spectroscopy of Fe-ZSM-5 ( $\left.v_{\text {ex }}=16,500 \mathrm{~cm}^{-1}\right), a$ feature at $867 \mathrm{~cm}^{-1}$ is observed that is not present before $\mathrm{N}_{2} \mathrm{O}$ activation, and decays in the presence of benzene. This feature has been assigned to $\alpha-O$, but is misattributed as the $\mathrm{O}-\mathrm{O}$ stretch of a binuclear oxo/peroxo-bridged diferric site. ${ }^{94}$ The ${ }^{16 / 18} \mathrm{O}$ sensitivity of the $867 \mathrm{~cm}^{-1}$ vibration feature has also not been evaluated, and it has not yet been 
shown to be in resonance with the $16500 \mathrm{~cm}^{-1}$ absorption band of $\alpha-\mathrm{O}$.

In low temperature MCD (figure 52, middle), the lowest energy absorption band of $\alpha-O$ in Fe-BEA appears as a weak negative feature in the $5,000-9,000 \mathrm{~cm}^{-1}$ region with fine structure ${ }^{35}$ For mononuclear non-heme $\mathrm{S}=2 \mathrm{Fe}(\mathrm{IV})=\mathrm{O}$ sites, the lowest energy MCD features are the $d \pi \rightarrow d_{z^{2}}$ ligand field excited states, which show fine structure due to vibronic progressions in the Fe-O stretch. ${ }^{198,199}$ However, these transitions generally occur at energies $>12,000 \mathrm{~cm}^{-1}$. The low energy of the $\alpha-O$ band may result from the stability of $d_{z^{2}}$ in the absence of a trans axial ligand, reflecting the entatic geometry of the active site. This possibility has not yet been evaluated. At higher energy, there is a positive band at $20,100 \mathrm{~cm}^{-1}$ with a low-energy shoulder at ca. $16900 \mathrm{~cm}^{-1}$. These MCD bands correspond to the DR-UV-vis features at 16,900 and $\sim 20,000 \mathrm{~cm}^{-1}$, but with an inverted intensity ratio.

Like $\alpha-\mathrm{Fe}(\mathrm{II})$, the MCD features of $\alpha-\mathrm{O}$ are temperature-dependent non-linear $B$ terms (see 5.2.2 and 3.3.2). ${ }^{35,116}$ This is evident from field dependence in the lowtemperature saturation limit of VTVH-MCD isofields for the $20,100 \mathrm{~cm}^{-1}$ transition shown in the bottom left of figure 52. VTVH-MCD isotherms (figure 52, bottom right) are nested due to the positively-signed axial zero-field splitting $\left(D=8 \pm 1 \mathrm{~cm}^{-1}\right)$ of the $S=2$ ground state of $\alpha-\mathrm{O}$. This value of $D$ is comparable to other $\mathrm{S}=2$ mononuclear $\mathrm{Fe}(\mathrm{IV})=\mathrm{O}$ sites, where spin-orbit coupling to low-lying triplet excited states contributes dominantly to the zero-field splitting. ${ }^{200}$ Importantly, VTVH-MCD indicates $\alpha-\mathrm{O}$ is not an $\mathrm{Fe}(\mathrm{III})-\mathrm{O}^{-}$ species as has been proposed extensively in the literature, as a $D=8 \pm 1 \mathrm{~cm}^{-1}$ is well outside the range possible for this electronic structure $\left(\mathrm{D}<2 \mathrm{~cm}^{-1}\right) \cdot{ }^{19,109}$ 
5.3.3 - Reactivity of $\alpha-0 . \alpha-O$ is remarkable for its ability to hydroxylate the strong, unactivated $\mathrm{C}-\mathrm{H}$ bonds of methane and benzene rapidly at room temperature, making $\alpha-O$ one of the most reactive metal-oxo sites known. Due to the extreme reactivity of $\alpha$ O, no experimental estimates of activation barriers for selective hydrocarbon oxidation currently exist. The barriers for methane hydroxylation here are significantly lower than those observed for the Cu-zeolites $\left(\Delta \mathrm{H}^{*}=11-16 \mathrm{kcal} / \mathrm{mol}\right.$ - see section 4.2), which react only sluggishly at room temperature. The H/D KIEs for methane and benzene hydroxylation have been evaluated in Fe-ZSM-5 using mixed-isotope substrates (e.g. $\mathrm{CH}_{2} \mathrm{D}_{2}$ and $\mathrm{C}_{6} \mathrm{H}_{3} \mathrm{D}_{3}$ - intramolecular KIEs are not susceptible to $\mathrm{H} / \mathrm{D}$ isotope effects on diffusion). ${ }^{33}$ The intramolecular $\mathrm{H} / \mathrm{D}$ KIE for $\mathrm{CH}_{2} \mathrm{D}_{2}$ hydroxylation is 1.9 at $100 \mathrm{C}$, increasing to 5.5 at $-50{ }^{\circ} \mathrm{C}$ (the temperature dependence of the KIE indicates an H/D $\Delta \Delta \mathrm{H}^{*}$ of $1.2 \pm 0.1 \mathrm{kcal} / \mathrm{mol}$ ), indicating the rate-limiting step in methane hydroxylation is $\mathrm{C}-\mathrm{H}$ cleavage. On the other hand, the intramolecular $\mathrm{H} / \mathrm{D}$ KIE for $\mathrm{C}_{6} \mathrm{H}_{3} \mathrm{D}_{3}$ hydroxylation is close to unity at $25^{\circ} \mathrm{C}(1.05)$, indicating $\mathrm{C}-\mathrm{H}$ cleavage does not occur in the ratelimiting step of benzene hydroxylation, characteristic of an electrophilic aromatic substitution mechanism (EAS, vide infra). For Fe-zeolites, the methane reaction is generally considered to be stoichiometric (although conditions enabling multiple turnovers have recently been reported). ${ }^{201} \alpha-\mathrm{Fe}-\mathrm{OH}$ and $\alpha-\mathrm{Fe}-\mathrm{OCH}_{3}$ sites have been identified by isotopically-perturbed IR studies following reaction with methane. ${ }^{202}$ The $\mathrm{OCH}_{3}$ species are described as product-trapped sites formed by methyl radical recombination with $\alpha-O$. These must be hydrolyzed with steam, releasing methanol. The benzene reaction on the other hand does not suffer from product trapping. ${ }^{181}$ There are however issues related to coke formation with aromatic substrates. 
Spectroscopically-validated DFT

models of $\alpha-\mathrm{O}$ have provided insight into how its entatic state (vide supra) leads to high reactivity with methane. ${ }^{35}$ Reacting $\alpha-\mathrm{O}(\mathrm{BEA})$ with $\mathrm{CH}_{4}$ in silico shows the first fundamental step in the hydroxylation reaction coordinate is $\mathrm{H}$-atom abstraction, which yields an $\mathrm{Fe}(\mathrm{III})-\mathrm{OH}$ product and a

methyl radical (figure 53). HAA occurs on the quintet surface with a low activation barrier of $3.6 \mathrm{kcal} / \mathrm{mol}$ (figure 53 , top)consistent with the room-temperature $\mathrm{CH}_{4}$
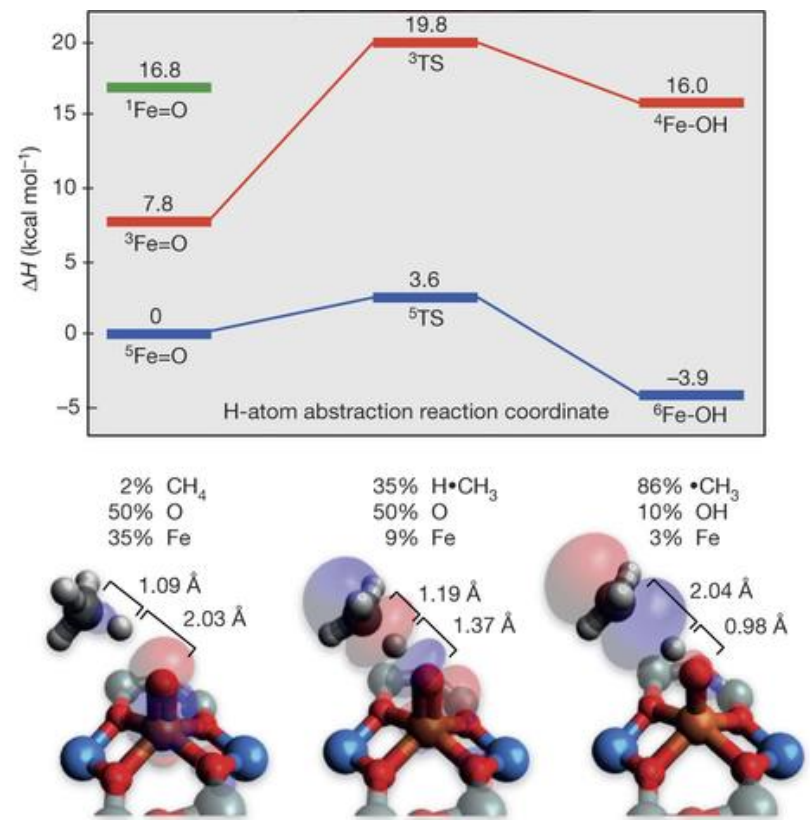

Figure 53. $\alpha-\mathrm{O} / \mathrm{CH}_{4}$ HAA abstraction reaction coordinate (top), including geometric structures and reactive FMOs for the reactants (bottom left), transition state (bottom middle), and products (bottom right). Adapted with permission from reference ${ }^{35}$. Copyright 2016 Nature Publishing Group

reactivity of $\alpha$-O. A similar barrier is predicted for Fe-ZSM-5. ${ }^{178}$ Two factors contribute to this exceptionally low barrier. First, the HAA reaction is nearly isoenthalpic, indicating the $\mathrm{O}-\mathrm{H}$ bond of the $\mathrm{Fe}(\mathrm{III})-\mathrm{OH}$ first product and the $\mathrm{C}-\mathrm{H}$ bond of $\mathrm{CH}_{4}$ are comparable in strength. There is therefore an unusually large driving force for $\mathrm{O}-\mathrm{H}$ bond formation with $\alpha-\mathrm{O}$, and this substantially lowers the barrier for HAA. The exceptional strength of the product $\mathrm{O}-\mathrm{H}$ bond is related to the entatic geometry of $\alpha-\mathrm{O}$ (see 5.3.1), which destabilizes the $\mathrm{S}=2 \mathrm{Fe}(\mathrm{IV})=\mathrm{O}$ active site over the $\mathrm{S}=5 / 2 \mathrm{Fe}(\mathrm{III})-\mathrm{OH}$ product, increasing the driving force for $\mathrm{O}-\mathrm{H}$ bond formation by about $6 \mathrm{kcal} / \mathrm{mol}$. This additional driving force would be quenched by adding a trans axial ligand, or by spin pairing (i.e. for the reaction on the $S=1$ surface, see top of figure 53). The enatic state of $\alpha-O$ also leads to high intrinsic reactivity in HAA. The site's entatic geometry (vacant trans axial position) 
stabilizes the $\mathrm{Fe}\left(3 \mathrm{~d}_{z^{2}}\right)$ orbital, resulting in a highly covalent $\mathrm{Fe}=\mathrm{O}$ unit. This, combined with a large exchange stabilization of the $\mathrm{Fe}(3 \mathrm{~d})$ manifold (associated with the $\mathrm{S}=2$ electronic structure), lead to an $\mathrm{Fe}(\mathrm{IV})=\mathrm{O}$ bond that strongly spin-polarizes towards an $\mathrm{Fe}(\mathrm{III})-\mathrm{O}^{--}$electronic structure at the transition-state geometry for HAA (where the FeO bond elongates from $1.59 \AA$ to $1.72 \AA$ ).

Unlike methane, the reaction of benzene with $\alpha-O$ (ZSM-5) results in the formation of a spectroscopic intermediate with abroad absorption feature centered at $14,500 \mathrm{~cm}^{-1} .{ }^{94}$ Using $16500 \mathrm{~cm}^{-1}$ laser excitation, Raman vibrations are observed that have been assigned to a ferric phenolate chromophore. How this spectroscopic intermediate fits with the catalytic cycle for benzene hydroxylation is not yet understood, although it has been proposed that this represents a product-trapped state. ${ }^{94}$ The reaction of $\alpha-\mathrm{O}$ with benzene has not yet been explored using a spectroscopicallyvalidated DFT model. For mononuclear $\mathrm{S}=2$ non-heme $\mathrm{Fe}(\mathrm{IV})=\mathrm{O}$ sites, the rate-limiting step in aromatic hydroxylation is believed to be the initial electrophilic attack of the oxo on the aromatic ring. ${ }^{196,203}$ This occurs through a radical EAS mechanism, forming a $\sigma$ complex with an $\mathrm{S}=5 / 2 \mathrm{Fe}(\mathrm{III})$ center. Whether $\alpha-\mathrm{O}$ follows this trend remains to be seen. In this case, the same factors that activate $\alpha-O$ for HAA would be relevant, as the radical EAS mechanism and HAA both generate an axial square pyramidal $\mathrm{S}=5 / 2 \mathrm{Fe}$ (III) first product.

5.4 - Selective Hydrocarbon Oxidation with $\mathrm{H}_{2} \mathrm{O}_{2}$. In addition to the gas-phase oxidation chemistry performed by Fe- and Cu-zeolites using $\mathrm{N}_{2} \mathrm{O}_{/} \mathrm{O}_{2}$, Fe-ZSM-5 has been shown to perform aqueous-phase methane hydroxylation using $\mathrm{H}_{2} \mathrm{O}_{2}$ at low 
temperature and high pressures (e.g. $50 \stackrel{\circ}{\circ}$ at 30.5 bar). ${ }^{92}$ In contrast to the gas-phase chemistry, the $\mathrm{H}_{2} \mathrm{O}_{2} / \mathrm{Fe}-\mathrm{ZSM}-5$ system is catalytic (TOF $=14,000 \mathrm{hr}^{-1}$ ). The initial steps in methane activation are also distinct here, forming $\mathrm{MeOOH}$ instead of $\mathrm{MeOH}$. (MeOH is formed via subsequent decomposition of the $\mathrm{MeOOH}$ first product.) It is believed that $\mathrm{MeOOH}$ formation proceeds via $\mathrm{H}$-atom abstraction. ${ }^{204}$ The activation of methane is unaffected by the presence of radical traps. This indicates free peroxyl or hydroxyl radicals (from Fenton chemistry) do not contribute to $\mathrm{MeOOH}$ formation, and that reactivity occurs at a bound $\mathrm{Fe} / \mathrm{O}$ or $\mathrm{Fe}-\mathrm{OOH}$ intermediate. $\mathrm{H}$-atom abstraction is proposed to be mediated by an activated $\mathrm{Fe} / \mathrm{O}$ intermediate with an inactive peroxide ligand that does not participate directly in $\mathrm{C}-\mathrm{H}$ cleavage. ${ }^{204}$ The methyl radical product of $\mathrm{H}$-atom abstraction is then proposed to recombine with the peroxide ligand to form $\mathrm{MeOOH}$. This represents a deviation from the mechanisms proposed for the $\mathrm{N} 2 \mathrm{O} / \mathrm{O}_{2}$ gas-phase oxidation of methane, where the methyl radical recombines directly with the active oxygen. In addition to $\mathrm{MeOOH}$ and $\mathrm{MeOH}$, higher oxygenates (formate and $\mathrm{CO}_{2}$, but not formaldehyde) are observed in the product distribution - sometimes contributing dominantly. This issue has been resolved with the addition of $\mathrm{Cu}$ as a co-cation. ${ }^{92}$ Methanol selectivities exceeding $95 \%$ have been achieved for this system, referred to as Cu-promoted Fe-ZSM-5. The role of $\mathrm{Cu}$ in improving selectivity is not currently understood.

The iron active site(s) in the $\mathrm{H}_{2} \mathrm{O}_{2} / \mathrm{Fe}-\mathrm{ZSM}-5$ system are extra-lattice species that are distinct from $\alpha-\mathrm{Fe}(\mathrm{II}) / \alpha$ -

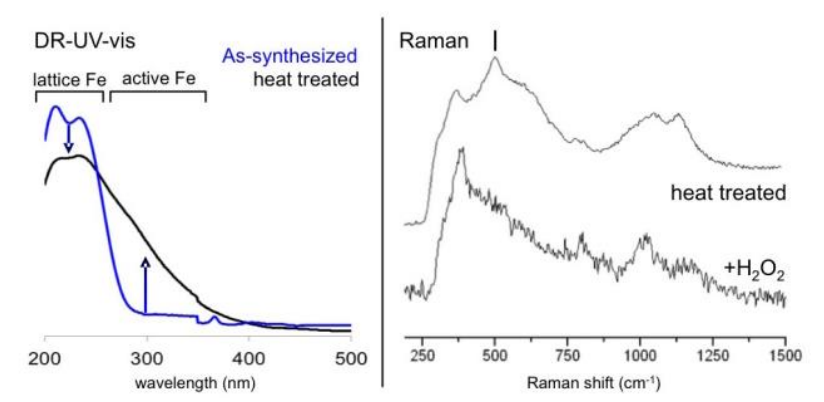

Figure 54. DR-UV-vis changes during heat treatment (left); and UV Raman features ( $\lambda_{\text {ex }}=325 \mathrm{~nm}$ ) correlated with $\mathrm{H}_{2} \mathrm{O}_{2}$ activation (right, marked peak eliminated). ${ }^{93,205}$ 
O. ${ }^{205}$ The nature of the active iron component and its activation by $\mathrm{H}_{2} \mathrm{O}_{2}$ are not currently understood. Active sites are generated during heat treatment of Fe-ZSM-5 containing isomorphously substituted lattice $\mathrm{Fe}$ (III) sites. This causes some $\mathrm{Fe}$ (III) to be extruded from lattice, forming cationic (extra-lattice) $\mathrm{Fe}(\mathrm{III})$ sites. The migration of $\mathrm{Fe}$ (III) is evident from DR-UV-vis spectral data shown in figure 54 (left). Tetrahedral lattice $\mathrm{Fe}(\mathrm{III})$ sites exhibit a pair of lattice $\rightarrow \mathrm{Fe}$ (III) CT transitions in the $40,000-50,000$ $\mathrm{cm}^{-1}$ region, and these decrease in intensity following heat treatment. ${ }^{205}$ This is associated with the growth of a new band centered around $33,000 \mathrm{~cm}^{-1}$ that is correlated with activity. While the peroxide sensitivity of this band has not yet been evaluated, tuning a laser $(325 \mathrm{~nm})$ into this absorption feature enhances a vibrations at $521 \mathrm{~cm}^{-1}$ that is not present before heat treatment and decays in the presence of peroxide (figure 54, right). ${ }^{93}$ This vibration may be related to the $\mathrm{Fe}$ (III) precursor to the reactive intermediate in $\mathrm{MeOOH}$ synthesis. Peroxide-induced loss of the $521 \mathrm{~cm}^{-1}$ vibration is correlated with growth of a new vibration at $631 \mathrm{~cm}^{-1}$ seen only with visible excitation $(514 \mathrm{~nm})$. It has been proposed that this is the $\mathrm{Fe}-\mathrm{O}$ stretch of a $\mathrm{Fe}(\mathrm{III})-\mathrm{OOH}$ site. This proposal has not been fully evaluated.

6. Correlation of Metallozeolites to Metalloenzymes. Parallels have been drawn between metallozeolite and metalloenzyme catalysis, particularly with respect to selective oxidation of methane. Analogies have been proposed between Cu-zeolites and the dicopper active site of particulate methane monooxygenase (pMMO), ${ }^{66,160,206}$ and between Fe-zeolites and the diiron active site of soluble methane monooxygenase 
(sMMO). ${ }^{33,94,202,207}$ These analogies can now be evaluated and amended based on emerging insight into metallozeolite and metalloenzyme active sites.

6.1 - Cu-Zeolites and pMMO. Particulate methane monooxygenase is an enzyme expressed across methanotrophs that performs the first step of their metabolism, oxidizing methane to methanol under

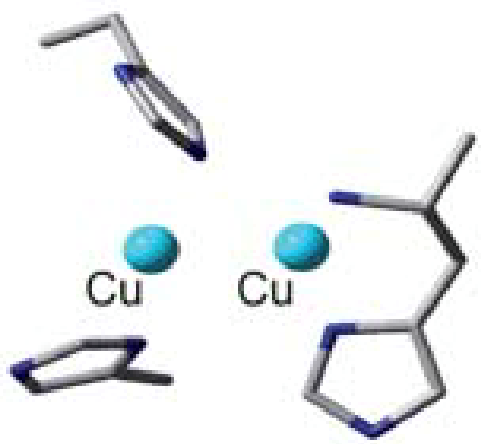

physiological conditions. ${ }^{21,208}$ Like the Cu-zeolites, Figure 55. Binuclear $\mathrm{Cu}$ active site of pMMO. Reproduced methane hydroxylation in pMMO is believed to occur at a $\begin{aligned} & \text { from reference }{ }^{212} \text { with } \\ & \text { permission from the Royal }\end{aligned}$ binuclear $\mathrm{Cu}$ active site. ${ }^{161,162}$ In resting (as isolated) Society of Chemistry. pMMO, crystallography and ${ }^{14} \mathrm{~N}$ ENDOR indicate bis-His ligation for one $\mathrm{Cu}$ center, while the other is bound by a terminal histidine and its amine (figure 55). ${ }^{162,209}$ No water/hydroxide ligand is detected by ESEEM. ${ }^{209}$ Finally, in the reduced $2 \mathrm{Cu}(\mathrm{I})$ state of the pMMO active site, EXAFS shows a short Cu-Cu separation of $2.65 \AA$ (versus $4.2 \AA$ for the geometry optimized reduced $2 \mathrm{Cu}(\mathrm{I})$ active site in Cu-zeolites). ${ }^{135,210}$ 
Adding $\mathrm{O}_{2}$ or peroxide to the reduced active site of pMMO results in formation of an absorption feature at $345 \mathrm{~nm}$ that decays in the presence of methane (figure 56). ${ }^{211}$ (Note that only the solubilized active protomer of pMMO reacts with $\mathrm{O}_{2}$, and not the full membrane-bound protein - an

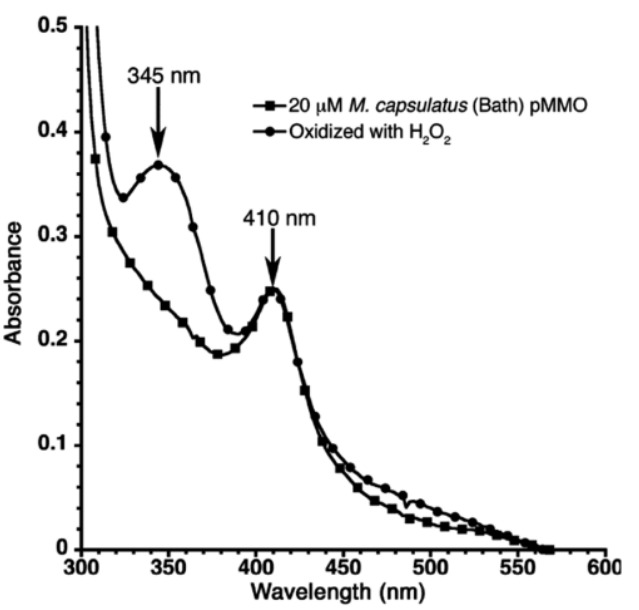
$\alpha_{3} \beta_{3} \gamma_{3}$ trimer which only reacts with $\mathrm{H}_{2} \mathrm{O}_{2}$ ). This Abs feature is suggested to derive from a $\mu-\eta^{2}: \eta^{2}$ peroxodicopper(II) intermediate, but this possibility

Figure 56. Comparison of absorption data before and after $\mathrm{H}_{2} \mathrm{O}_{2}$ binding in solubilized pMMO from Methyloc. capsulatus (Bath) (absorption at $410 \mathrm{~nm}$ due to heme contaminants). Reproduced from reference 211 with permission. Copyright 2012, American has not been evaluated experimentally. It is also not Chemical Society.

yet understood how the $\mathrm{O}_{2} / \mathrm{H}_{2} \mathrm{O}_{2}$-derived species relates to the reactive intermediate in methanol synthesis. In Cu-ZSM-5, a $345 \mathrm{~nm}$ Abs feature also forms in the presence of $\mathrm{O}_{2}$ at room temperature, and using isotopically-perturbed $r R$ spectroscopy, this was shown to correspond to a $\mu-\eta^{2}: \eta^{2}$ peroxodicopper(II) species that upon heating converts to the $\left[\mathrm{Cu}_{2} \mathrm{O}\right]^{2+}$ reactive intermediate (see section 4.3). ${ }^{136}$ Since the $\left[\mathrm{Cu}_{2} \mathrm{O}\right]^{2+}$ cores in Cu-zeolites are the only spectroscopically characterized dicopper sites that react with methane, there is interest in evaluating whether a $\left[\mathrm{Cu}_{2} \mathrm{O}\right]^{2+}$ core is a viable intermediate in pMMO

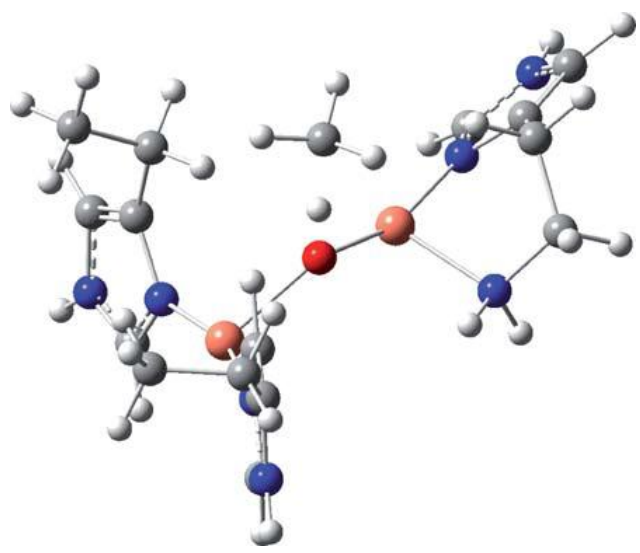
catalysis. The methane reactivity of a Figure 57. Transition state structure for $\mathrm{H}$-atom abstraction by a $\left[\mathrm{Cu}_{2} \mathrm{O}\right]^{2+}$ core stabilized at the $\left[\mathrm{Cu}_{2} \mathrm{O}\right]^{2+}$ core stabilized in the pMMO active active site of $\mathrm{pMMO}$. Reproduced from reference ${ }^{212}$ with permission from the Royal Society of Chemistry. 
site has been explored using DFT, and like Cu-ZSM-5, it was found that polarization toward a $2 \mathrm{Cu}(\mathrm{I} / \mathrm{II})$-oxyl electronic structure was key to the high reactivity of this model (figure 57)..212 The lack of spectroscopic data on the reactive intermediate in $\mathrm{pMMO}$ makes it difficult to evaluate this proposal, however.

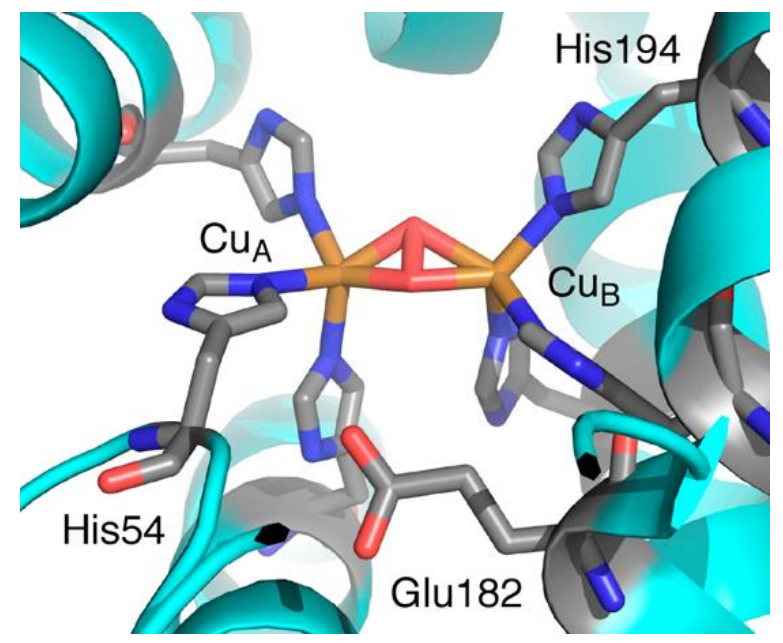

A more complete comparison can be made to the binuclear Cu enzyme tyrosinase,

Figure 58. Active site of oxytyrosinase. Adapted from reference 18 with permission. Copyright 2014, American Chemical Society.

where extensive spectroscopic and structural data already exist. ${ }^{18}$ Tyrosinase activates $\mathrm{O}_{2}$ to hydroxylate monophenols and oxidize the resulting diphenols to quinones. The active site of tyrosinase ligates each $\mathrm{Cu}$ center with three His residues (figure 58), and in the reduced active site the Cu centers are separated by ca. $4 \AA$ - similar to the $\mathrm{Cu}$ -
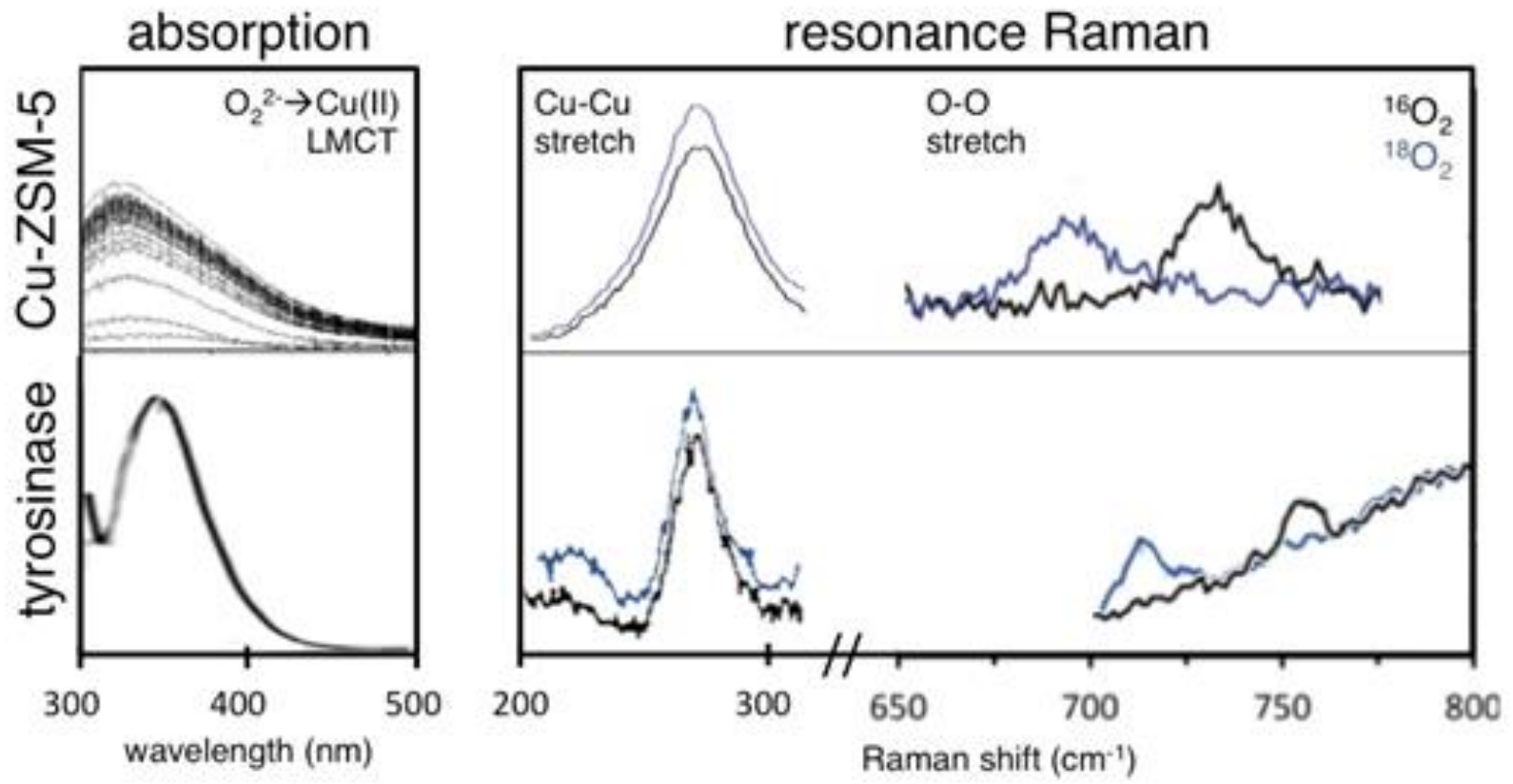

Figure 59. Comparison of absorption (left) and ${ }^{16 / 18} \mathrm{O}$-perturbed resonance Raman spectra (right, $\lambda_{\text {ex }}=364$ $\mathrm{nm}$ ) of the $\left[\mathrm{Cu}_{2}\left(\mathrm{O}_{2}\right)\right]^{2+}$ cores of oxy-Cu-ZSM-5 (top) and oxytyrosinase (bottom). 
zeolites. ${ }^{135,213}$ Like Cu-ZSM-5, $\mathrm{O}_{2}$ binds to the reduced active site in a bridging peroxo fashion, forming a side-on $\mu-\eta^{2}: \eta^{2}$ peroxodicopper(II) species that is the reactive intermediate in aromatic hydroxylation. ${ }^{213}$ Both the low temperature oxy-Cu-ZSM-5 and oxytyrosinase intermediates are associated with intense $29,000 \mathrm{~cm}^{-1} \mathrm{O}_{2}{ }^{2-} \rightarrow \mathrm{Cu}$ (II) CT transitions, and tuning a laser into these charge transfer bands enhances similar $r R$ vibrations (figure 59). ${ }^{136,214}$ For oxytyrosinase, the O-O stretch of the peroxide core appears at $755 \mathrm{~cm}^{-1}\left(\Delta^{16 / 18} \mathrm{O}_{2}=41 \mathrm{~cm}^{-1}\right)$, along with an isotope insensitive $\mathrm{Cu}-\mathrm{Cu}$ stretch at $274 \mathrm{~cm}^{-1} .{ }^{214,215}$ For Cu-ZSM-5, analogous features appear at $736 \mathrm{~cm}^{-1}$ $\left(\Delta^{16 / 18} \mathrm{O}_{2}=41 \mathrm{~cm}^{-1}\right)$ and $269 \mathrm{~cm}^{-1}$. The geometric and electronic structures of these sites are therefore highly similar. The activation of the Cu-ZSM-5 peroxo core for aromatic hydroxylation has not yet been explored, and it will be interesting to see how this compares to oxytyrosinase. However in contrast to the enzyme, in the zeolite there are spectator $\mathrm{Cu}$ sites that can donate two electrons to the $\mu-\eta^{2}: \eta^{2}$ peroxodicopper(II) intermediate to reductively cleave the $\mathrm{O}-\mathrm{O}$ bond and generate a $\left[\mathrm{Cu}_{2} \mathrm{O}\right]^{2+}$ core. $^{136}$

A second role fulfilled by binuclear active sites in Cu-zeolites, in addition to selective hydrocarbon oxidation, is reduction of $\mathrm{N}_{2} \mathrm{O} \cdot{ }^{37,135}$ While the reactivity of pMMO with $\mathrm{N}_{2} \mathrm{O}$ has not been explored, direct comparisons can be made between Cu-zeolites and nitrous oxide reductase $\left(\mathrm{N}_{2} \mathrm{OR}\right)$, a Cu-containing enzyme that catalyzes reduction of

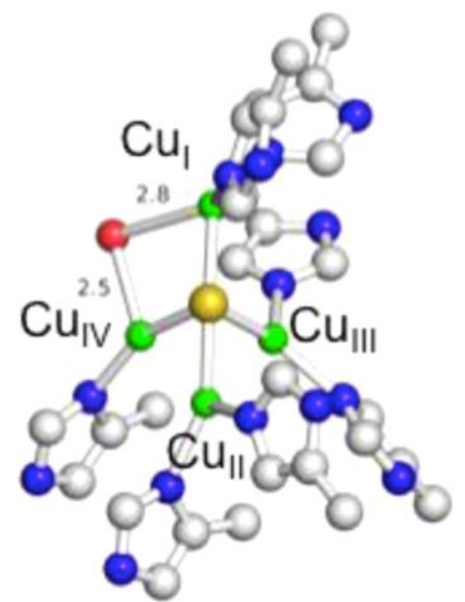

Figure 60. Resting structure of $\mathrm{Cu}_{z}$ cluster from $P$. denitrificans $\mathrm{N}_{2} \mathrm{OR}$ (purified aerobically).217 Copper is colored in green, sulfide in yellow, iodine in purple, and oxygen in red. Adapted from reference 18 with permission. Copyright 2014, American Chemical Society. 
$\mathrm{N}_{2} \mathrm{O}$ to $\mathrm{N}_{2}$ and $\mathrm{H}_{2} \mathrm{O}$ in the final step in bacterial denitrification. ${ }^{216}$ The active site of $\mathrm{N}_{2} \mathrm{OR}$ is a tetranuclear $\mathrm{Cu}_{4} \mathrm{~S}$ cluster $\left(\mathrm{Cu}_{z}\right)$, which features a $\mu 4$-sulfide and $\mathrm{Cu}$ ligation provided by histidine residues (figure 60). ${ }^{217,218}$ The reduced $4 \mathrm{Cu}(\mathrm{l})$ form of $\mathrm{Cu}_{z}$ has a vacancy on the CulCuiv edge that is the site of $\mathrm{N}_{2} \mathrm{O}$ activation. ${ }^{218,219}$ As with Cu-ZSM-5, the
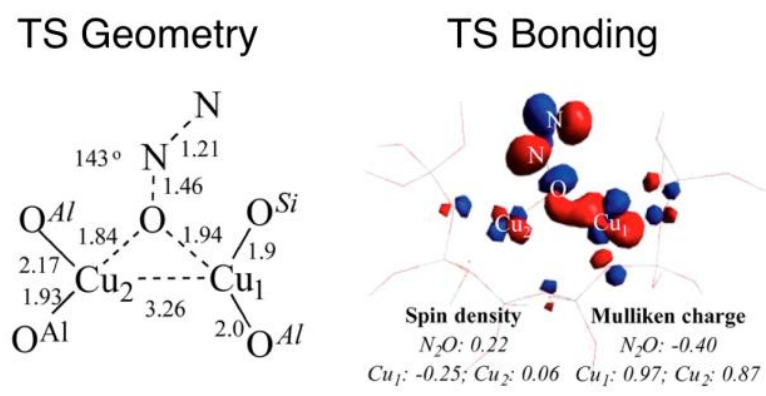
ability of $\mathrm{N}_{2} \mathrm{O}$ to bridge the adjacent
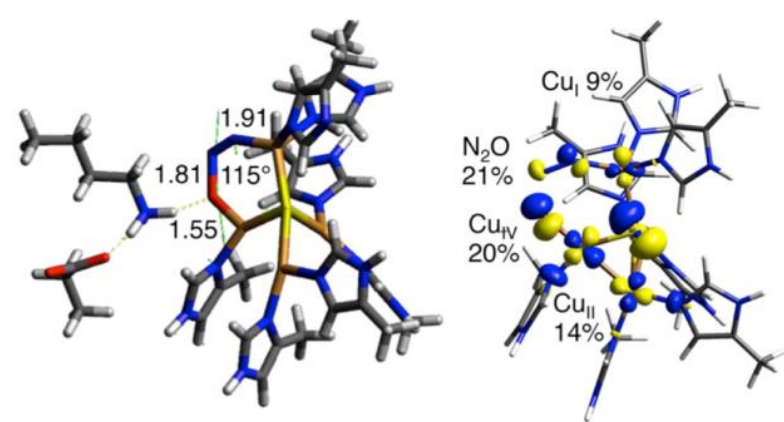

$\mathrm{Cu} /$ CuIv centers is key to function,

Figure 61. Comparison of transition state structures (left) and bonding (right) for $\mathrm{N}-\mathrm{O}$ cleavage by reduced Cu-ZSM-5 (top) and reduced Cuz (bottom).

facilitating 2e- reduction during $\mathrm{N}-\mathrm{O}$ cleavage (figure 61 ). ${ }^{135,151,219}$ The mechanisms of $\mathrm{N}_{2} \mathrm{OR}$ and $\mathrm{Cu}-\mathrm{ZSM}-5$ are not comparable, however. In Cu-ZSM-5, N-O cleavage is calculated to proceed from a $\mu-1,1-\mathrm{O}$ bridged intermediate, where sequential transfer of two electrons from different copper centers to cleave the $\mathrm{N}-\mathrm{O}$ bond is driven by the formation of strong $\mathrm{Cu}(\mathrm{II})-\mathrm{oxo}$ bonds. ${ }^{135}$ In $\mathrm{N}_{2} \mathrm{OR}, \mathrm{N}_{2} \mathrm{O}$ bridges in a $\mu-1,3-\mathrm{O}, \mathrm{N}$ fashion and a proton is transferred from a second-sphere residue to the $\mathrm{O}$ atom of $\mathrm{N}_{2} \mathrm{O}$ during $\mathrm{N}-\mathrm{O}$ cleavage (figure 61 , bottom), and the electrons are transferred sequentially through one $\mathrm{Cu}$ of the $\mathrm{Cu}_{z}$ cluster to $\mathrm{N}_{2} \mathrm{O} .{ }^{151}$ The driving force for $\mathrm{O}-\mathrm{H}$ bond formation is therefore an important contribution to $\mathrm{N}-\mathrm{O}$ cleavage in $\mathrm{N}_{2} \mathrm{OR}$. This highlights an important difference between enzyme and zeolite catalysis. Whereas proton-coupled electron transfer and sequential proton/electron transfer mechanisms are commonplace 
in biology, protons are not readily available in gas-phase metallozeolite chemistry and likely do not contribute to reactivity.

6.2 - Fe-Zeolites and sMMO. Soluble methane monooxygenase (sMMO) is a diiron enzyme expressed across methanotrophs that oxidizes methane to form methanol using atmospheric $\mathrm{O}_{2}{ }^{21}$ Based on reactivity, structural analogies have been proposed between sMMO and the active site of methane hydroxylation in Fe-zeolites. ${ }^{33,94,202,207}$ Combined results from crystallography and spectroscopy have defined the geometric and electronic structure of key catalytic intermediates in sMMO. In contrast to the mononuclear active site of the Fe-zeolites, the sMMO active site is binuclear. The reduced $2 \mathrm{Fe}(\mathrm{II})$ state features two five-coordinate $\mathrm{Fe}$ centers with histidine and carboxylate ligation, along with two bridging carboxylates, one binding in a $\mu-1,3$ fashion, the other binding $\mu-1,1$ (figure 62 , left). ${ }^{220} \mathrm{O}_{2}$ binds to the vacant coordination positions of the $2 \mathrm{Fe}(\mathrm{II})$ active site forming intermediate $\mathrm{P}$, a cis- $\mu-1,2$ peroxodiferric site

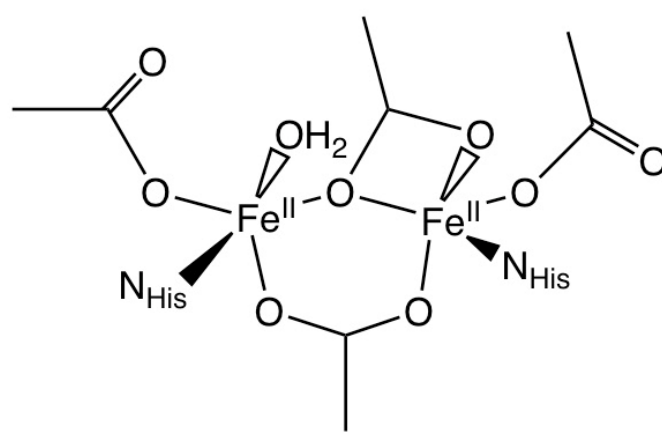

reduced SMMO active site

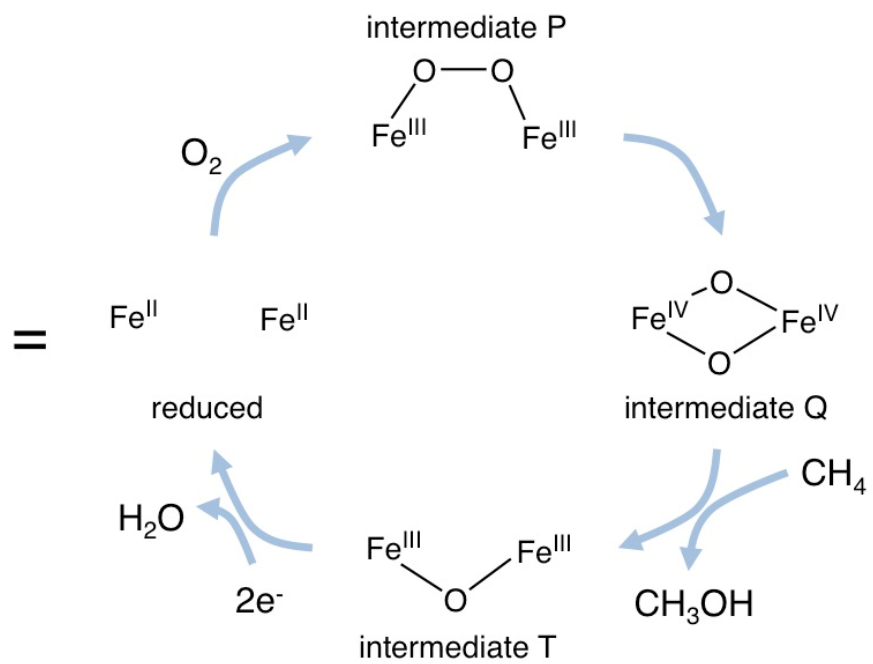

Figure 62. Structure of the reduced SMMO active site (left), along with key intermediates in catalysis (right). 
(figure 62, right). ${ }^{221}$ This converts to the active species in methanol synthesis, the highvalent bis- $\mu$-oxo diferryl intermediate $Q$, which features antiferromagnetically-coupled high spin $\mathrm{Fe}(\mathrm{IV})$ centers. ${ }^{222,223}$ Intermediate $\mathrm{Q}$ reacts rapidly with methane at room temperature $(\Delta \mathrm{H}=8.5 \pm 0.3 \mathrm{kcal} / \mathrm{mol})$ with a $\mathrm{H} / \mathrm{D}$ KIE of $23 \pm 1$ indicating $\mathrm{C}-\mathrm{H}$ cleavage (with tunneling) as the rate-limiting step. 224 This forms methanol, as well as the oxobridged diferric intermediate T. ${ }^{221}$

The ability to bind and reduce $\mathrm{O}_{2}$ by four electrons is key to reactivity in sMMO. This is in contrast to Fe-zeolites, where the reduced $\alpha-\mathrm{Fe}(\mathrm{II})$ active site does not bind $\mathrm{O}_{2},{ }^{32}$ but does bind and reduce $\mathrm{N}_{2} \mathrm{O}$ by two electrons to form $\alpha-\mathrm{O}$, a mononuclear $\mathrm{S}=2$ terminal $\mathrm{Fe}(\mathrm{IV})=\mathrm{O}$ species. ${ }^{35}$ The geometric and electronic structures of $\alpha-\mathrm{O}$ and sMMO $\mathrm{Q}$ are distinct, and there are differences in the strategies used by each site to achieve high reactivity. Whereas $\alpha-O$ is a mononuclear site held in a reactive 'entatic' geometry by the rigid zeolite lattice (see section 6.3 .3 for detail), ${ }^{35}$ the reactivity of $s M M O Q$ is related to the intrinsic activation of the $\left[\mathrm{Fe}_{2} \mathrm{O}_{2}\right]^{4+}$ diamond core motif having two antiferromagnetically coupled high spin $\mathrm{Fe}(\mathrm{IV})$ centers. Like $\alpha-\mathrm{O}$, it is likely that one of the bridging oxos in sMMO Q evolves oxyl character at the methane HAA transition state geometry. ${ }^{225}$ However the mechanism of FMO polarization, the driving force for $\mathrm{O}$ $\mathrm{H}$ bond formation, and how these relate to the coupling between the Fe centers has not yet been presented.

For $\alpha-\mathrm{O}$, a more direct comparison to biology can be made with the mononuclear $\mathrm{S}=2 \mathrm{Fe}(\mathrm{IV})=\mathrm{O}$ active intermediates in the $\alpha$-keto acid-dependent non-heme Fe enzymes 4-hydroxyphenylpyruvate dioxygenase (HPPD) and 4-hydroxymandelate synthase (HmaS). ${ }^{226,227}$ HPPD and HmaS have comparable active site structures and act on the 

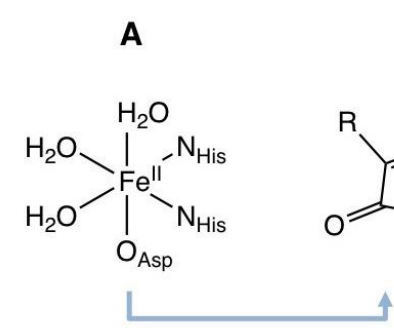

$-3 \mathrm{H}_{2} \mathrm{O},+\mathrm{HPP}$
B

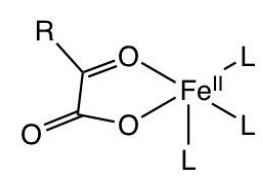

C

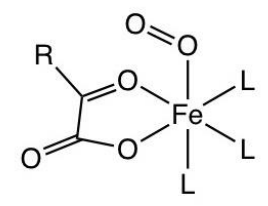

D

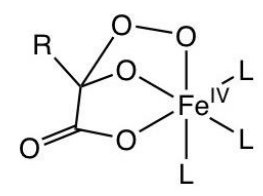

$E$



Figure 63. Analogous catalytic intermediates for HPPD and HmaS ( $R=4$-hydroxybenzyl). same substrate (4-hydroxyphenylpyruvate - HPP), but react differently: HPPD performs electrophilic aromatic hydroxylation, while HmaS performs aliphatic hydroxylation through $\mathrm{H}$-atom abstraction. This difference in function is ascribed to differences in the orientation of the substrate by the HPPD/HmaS active site pockets, and not to differences in intrinsic reactivity of the $\mathrm{Fe}(\mathrm{IV})=\mathrm{O}$ cores. ${ }^{196} \mathrm{HPPD} / \mathrm{HmaS}$ can therefore be compared directly to $\alpha-\mathrm{O}$, which is also a mononuclear $\mathrm{S}=2 \mathrm{Fe}(\mathrm{IV})=\mathrm{O}$ intermediate active in aliphatic and aromatic hydroxylation. ${ }^{33,35}$

For HPPD/HmaS, the reduced Fe(II) form of the active site is six-coordinate, with three protein-derived ligands ( 2 His, 1 carboxylate) and three weakly coordinated waters (figure 63A). ${ }^{228,229}$ The HPP substrate binds in a bidentate fashion to the reduced active site, displacing two bound water molecules, and labilizing the third (figure 63B). This results in a mixture of water-unbound (five-coordinate) and bound (six-coordinate) forms. ${ }^{230}$ The coordinatively unsaturated five-coordinate site then reacts with $\mathrm{O}_{2}$ (figure 63C). It is believed that this forms a transient $\mathrm{FeO}_{2}$ species that nucleophilically attacks the $\alpha$ carbon of the $\alpha$-keto acid substrate, forming a peroxide bridge that subsequently decays through oxidative decarboxylation to generate an $\mathrm{S}=2 \mathrm{Fe}(\mathrm{IV})=\mathrm{O}$ reactive intermediate with a monodentate-bound hydryoxyphenyl acetate (HPA; figure 63D,E). ${ }^{196}$

For these $\mathrm{Fe}(\mathrm{IV})=\mathrm{O}$ intermediates, two different reaction pathways are possible depending on the orientation of the benzyl group of the HPA substrate (R in figure 63) 
imposed by the active site protein pocket. ${ }^{196}$

For HPPD, the ipso carbon of the HPA aromatic ring is oriented along the $\mathrm{d}_{z^{2}}$-derived $\alpha$ LUMO of the $\mathrm{S}=2 \mathrm{Fe}(\mathrm{IV})=\mathrm{O}$ core, which has significant $(28 \%) O\left(p_{z}\right)$ character (figure 64, top left). This orbital pathway for reactivity defines the $\sigma$ channel in HPPD, which is associated with a $16 \mathrm{kcal} / \mathrm{mol}$ predicted barrier for $\mathrm{C}-\mathrm{O}$ bond formation (transition state shown in figure 64, top right). ${ }^{196}$ For HmaS, a benzylic hydrogen of HPA is oriented along a $d_{\pi}$-derived $\beta$ LUMO, which has significant $(32 \%) O\left(p_{\pi}\right)$ character
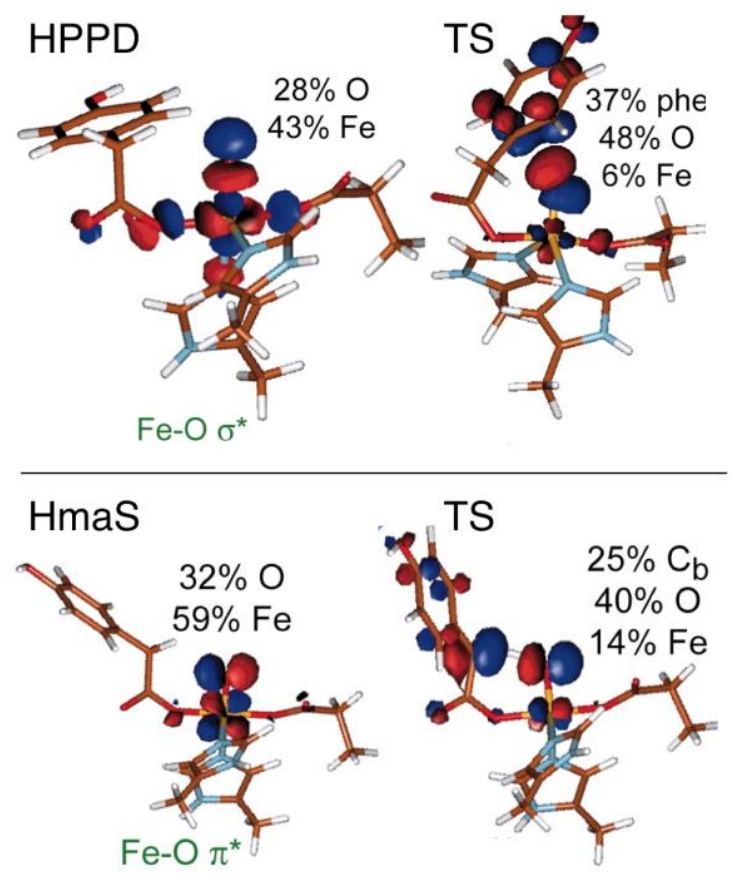

Figure 64. The active sites of HPPD (top) and HmaS (bottom) orient different atoms of the substrate toward different reactive molecular orbitals of the $\mathrm{Fe}(\mathrm{IV})=0$ core, resulting in different reactivity (electrophilic aromatic hydroxylation - top right, $\mathrm{H}$-atom abstraction bottom right). Adapted from reference ${ }^{196 .}$ Copyright 2006, National Academy of Sciences.

(figure 64, bottom left). This defines the $\pi$ channel in HmaS, which is associated with a $14 \mathrm{kcal} / \mathrm{mol}$ predicted barrier for $\mathrm{H}$-atom abstraction (transition state shown in figure 64 , bottom right). Thus low barriers are accessible through both the $\sigma$ and $\pi$ channels, and in both cases an important contribution to reactivity is evolution of ferric-oxyl character with $\mathrm{Fe}=\mathrm{O}$ elongation at the transition state. ${ }^{196}$ 
Compared to HPPD/HmaS, the $\mathrm{S}=2$ $\mathrm{Fe}(\mathrm{IV})=\mathrm{O}$ core of $\alpha-\mathrm{O}$ is significantly more reactive due to its constrained geometry. ${ }^{35}$ The enzyme intermediates are predicted to have square pyramidal geometry with an equatorial oxo ligand, ${ }^{196}$ and this is the most
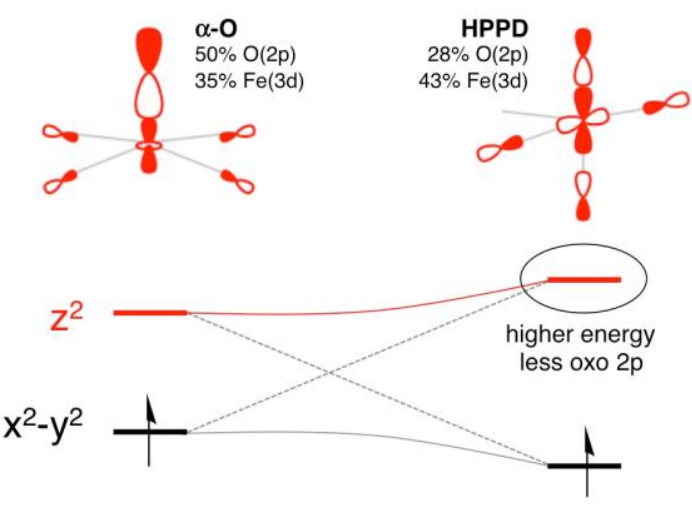
stable geometry for a 5-coordinate $S=2$ $\mathrm{Fe}(\mathrm{IV})=\mathrm{O}$ site. ${ }^{196,197}$ In contrast, the axial

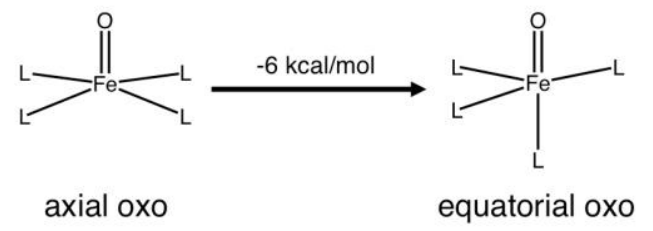

square pyramidal geometry of $\alpha-O$ is inherently unstable (figure 51), and is

Figure 65. Variation of the reactive LUMO (red) of a square pyramidal $\mathrm{S}=2 \mathrm{Fe}(\mathrm{IV})=\mathrm{O}$ site upon shifting the oxo ligand from the axial (left) to the equatorial position (right).

enforced by rigid constraints from the zeolite lattice. ${ }^{35}$ Differences in geometric structure lead to differences in electronic structure and thus reactivity. With respect to $\mathrm{H}$-atom abstraction, placing the oxo in the axial position destabilizes the $\mathrm{S}=2 \mathrm{Fe}(\mathrm{IV})=\mathrm{O}$ over the $\mathrm{S}=5 / 2 \mathrm{Fe}(\mathrm{III})-\mathrm{OH}$ product, increasing the driving force for $\mathrm{O}-\mathrm{H}$ bond formation. ${ }^{35}$ As shown in figure 65 , this geometry also leads to higher $\mathrm{Fe}=\mathrm{O}$ covalency, resulting in a more stable $\alpha$-LUMO with higher $O\left(p_{z}\right)$ content $(50 \%$ for $\alpha$-O versus $28 \%$ for HPPD). ${ }^{35,196}$ The $\alpha-O$ electronic structure also evolves ferric-oxyl character at shorter Fe-O distances relative to HmaS, resulting in a significantly lower barrier for HAA from a much stronger $\mathrm{C}-\mathrm{H}$ bond $(14 \mathrm{kcal} / \mathrm{mol}$ barrier for a $\sim 90 \mathrm{kcal} / \mathrm{mol}$ benzylic $\mathrm{C}-\mathrm{H}$ bond for HmaS versus a $4 \mathrm{kcal} / \mathrm{mol}$ barrier for a $104 \mathrm{kcal} / \mathrm{mol}$ methane $\mathrm{C}-\mathrm{H}$ bond for $\alpha-O) .{ }^{35,196}$

With respect to electrophilic aromatic hydroxylation, it is known that $\alpha-O$ is extremely reactive, converting benzene to phenol under mild reaction conditions. ${ }^{33,94,181,182}$ The molecular-level details of the reaction mechanism for $\alpha-O$ 
have not yet been determined. Whereas the approach of methane along the $\pi$ channel is sterically unencumbered, the $\sigma$ channel may be important for the more sterically demanding benzene substrate. When experimental insight into the benzene reaction becomes available, it will be interesting to draw comparisons to electrophilic aromatic hydroxylation by HPPD - particularly with respect to $\sigma$ versus $\pi$ channel reactivity, which has not yet been evaluated experimentally or computationally for $\alpha-\mathrm{O}$.

\section{3 - From Bioinorganic to Zeolite Catalysis: Tuning the Reactivity of Metal Active}

Sites. While metallozeolite and metalloenzyme active sites are not structurally comparable (vide supra), there are important parallels regarding their activation for reactivity. These include high metal-oxygen covalency (6.3.1), tuning of reactivity by second-sphere atoms (6.3.2), and entatic effects (6.3.3). These parallels, emerging from recent spectroscopic insight into metallozeolite and metalloenzyme active sites, highlight potentially useful mechanisms to tune the reactivity of catalysts.

6.3.1 - Metal-Oxygen Covalency. Reactive oxygen intermediates across metalloenzymes and metallozeolites exhibit spectroscopic features reflecting high metal-oxygen covalency (e.g. intense, low energy oxygen $\rightarrow$ metal charge transfer transitions in absorption spectroscopy). ${ }^{18,19}$ Combined spectroscopic and computational studies have shown that this is an important contribution the activation of these intermediates, increasing the oxygen $2 p$ content of the unoccupied metal $3 d$-derived orbitals that participate in electrophilic reactivity. Nature has therefore evolved active 
site architectures that lead to highly covalent $\mathrm{Cu}$ and non-heme $\mathrm{Fe}$ reactive oxygen intermediates.

For Fe enzymes, these involve ligands that donate strongly enough to stabilize high-valent oxidation states (e.g. ferryl iron), but also weakly enough to stabilize highspin electronic structures. Zeolites can also perform this balancing act, stabilizing highvalent high-spin $\mathrm{Fe}(\mathrm{IV})=\mathrm{O}$ intermediate using a weak zeolite lattice ligand field $(\alpha-\mathrm{O}) .{ }^{35}$ For both the Fe enzymes and zeolites, high oxidation states are associated with electron-deficient metal centers, and exogenous oxygen ligands will donate strongly to compensate for this, resulting in high metal-oxygen covalency and low-lying unoccupied acceptor orbitals. For the mononuclear $\mathrm{Fe}(\mathrm{IV})=\mathrm{O}$ intermediates, a high spin electronic structure makes it possible to achieve low barriers through covalency-activated $\pi$ and $\sigma$ channels. ${ }^{35,131,196,199,200}$ (This is in contrast to $\mathrm{S}=1 \mathrm{Fe}(\mathrm{IV})=\mathrm{O}$ intermediates, where only the $\pi$ channel is accessible). ${ }^{196}$ For the binuclear $\left[\mathrm{Fe}_{2}(\mathrm{O})_{2}\right]^{4+}$ intermediate $\mathrm{Q}$ in sMMO, it is not yet understood how the exchange coupled high spin state of the $\mathrm{Fe}(\mathrm{IV})$ centers relates to the activation of this site for $\mathrm{H}$-atom abstraction from methane.

For both the $\mathrm{Cu}$ enzymes and Cu-zeolites, there is no evidence that high-valent $\mathrm{Cu}$ (III) contributes to catalysis. Instead, high reactivity is achieved with $\mathrm{Cu}$ (II) species. ${ }^{18}$ For example, a direct comparison can be made between oxytyrosinase and oxy-CuZSM-5, which contain highly similar $\mu-\eta^{2}: \eta^{2}$ peroxodicopper(II) cores (vide supra). For these sites, high covalency is achieved through good orbital overlap and energy match between the filled $\pi^{*}$ orbitals of peroxide and the $3 d_{x^{2}-y^{2}}$ holes of the $\mathrm{Cu}(I I)$ centers. The role of the zeolite lattice in tuning the covalency of the mono-oxo $\left[\mathrm{Cu}_{2} \mathrm{O}\right]^{2+}$ site is different, however, and not comparable to the Cu enzymes. In this case, the $O(2 p)$ 
orbitals of the bridging oxo are stabilized relative to a bridging peroxide ligand. To counteract this, the zeolite lattice keeps the $\mathrm{Cu}$ (II) centers under-coordinated, and thus electron deficient. This brings the unfilled $3 d$ orbitals of the $\mathrm{Cu}(\mathrm{II})$ centers closer in energy to the filled $\mathrm{O}(2 \mathrm{p})$ orbitals, tuning up covalency. (This is in contrast to small molecule $\left[\mathrm{Cu}_{2} \mathrm{O}\right]^{2+}$ sites in the literature, which are coordinated by three or more strongly-donating nitrogen ligands, and display significantly reduced reactivity.) ${ }^{231-235}$ While the use of a low coordination number to achieve a high covalency is not significant in $\mathrm{Cu} / \mathrm{O}$ biochemistry, ${ }^{18}$ this is a key facet of the the blue Cu electron transfer site (see 6.3.3). ${ }^{117,121}$

6.3.2 - Second Sphere Effects. Atoms outside of the first coordination sphere can contribute significantly to reactivity in metalloenzyme active sites. This was seen in 6.1 , where $\mathrm{N}_{2} \mathrm{OR}$ facilitates $\mathrm{N}-\mathrm{O}$ cleavage using a proton transferred from a second-sphere lysine residue, ${ }^{151}$ and also in 6.2 , where the steric environments of the active site pockets of HPPD/HmaS guide reactivity through substrate orientation effects. ${ }^{196}$ The latter effect - second sphere influences based on sterics - may have direct parallels in metallozeolite chemistry. ${ }^{134}$ This builds on the concept of shape-selective catalysis, ${ }^{236}$ where the zeolite lattice and protein fold play analogous roles in guiding entry of substrate and release of product. For zeolites, this has mostly focused on the role of the lattice in tuning product distributions, especially with respect to hydrocarbon isomerization and alkylation catalyzed by acid sites. Recent studies of Cu-MOR have extended this concept to include second-sphere influence on the reactivity of metal sites in zeolites. ${ }^{134}$ In Cu-MOR (Si/Al=5), two $\left[\mathrm{Cu}_{2} \mathrm{O}\right]^{2+}$ cores are present with highly similar 
absorption and resonance Raman spectral features, and thus highly similar geometric and electronic structures. The reactivity of these sites with $\mathrm{CH}_{4}$ is distinct, however. One site has kinetic parameters similar to Cu-ZSM-5 $\left(\Delta \mathrm{H}^{\ddagger}=15 \mathrm{kcal} / \mathrm{mol}, \Delta \mathrm{S}^{\ddagger}=-36\right.$ $\mathrm{cal} / \mathrm{molK})$, while the other has a lower barrier $\left(\Delta \mathrm{H}^{\ddagger}=11 \mathrm{kcal} / \mathrm{mol}\right)$ and larger entropy of activation $\left(\Delta S^{\ddagger}=-44 \mathrm{cal} / \mathrm{molK}\right)$. Spectroscopy shows this difference in reactivity does not derive from differences in electronic structure. Instead, it was observed that site with the smaller $\Delta \mathrm{H}^{\ddagger}=11 \mathrm{kcal} / \mathrm{mol}$ has a larger $\Delta \mathrm{S}^{\ddagger}=-44$, and this entropic compensation is consistent with the confined reaction environment predicted for this site (buried in the side pockets or compressed 8-MR channels of the MOR lattice). Here, there is a direct parallel to the coupled binuclear $\mathrm{Cu}$ enzymes hemocyanin, tyrosinase, and catechol oxidase. ${ }^{18}$ These stabilize $\mu-\eta^{2}: \eta^{2}$ peroxodicopper(II) cores with highly similar spectral features yet different reactivity based on how the active site pocket guides the approach of substrate. The nature of the second-sphere effect in Cu-MOR is not yet understood, however. This may be related to steric effects, or alternatively to direct participation of second-sphere atoms in tuning the HAA reaction coordinate.

6.3.3 - The Entatic State. The entatic concept evolved from studies of metalloenzyme active sites, where protein constraints enforce an otherwise unstable geometry that is associated with high activity. ${ }^{118}$ This was first defined for the type 1 'blue' $\mathrm{Cu}$ active site of biological electron transfer found in plastocyanin and the multicopper oxidases, among others (see figure 66, top right). ${ }^{117}$ This is a mononuclear Cu center with trigonal pyramidal geometry, where the trigonal plane is defined by two histidine ligands and a cysteinate with a short $(2.13 \AA)$, covalent Cu-S bond. ${ }^{117}$ At $\sim 2.9 \AA$, there is an axial 


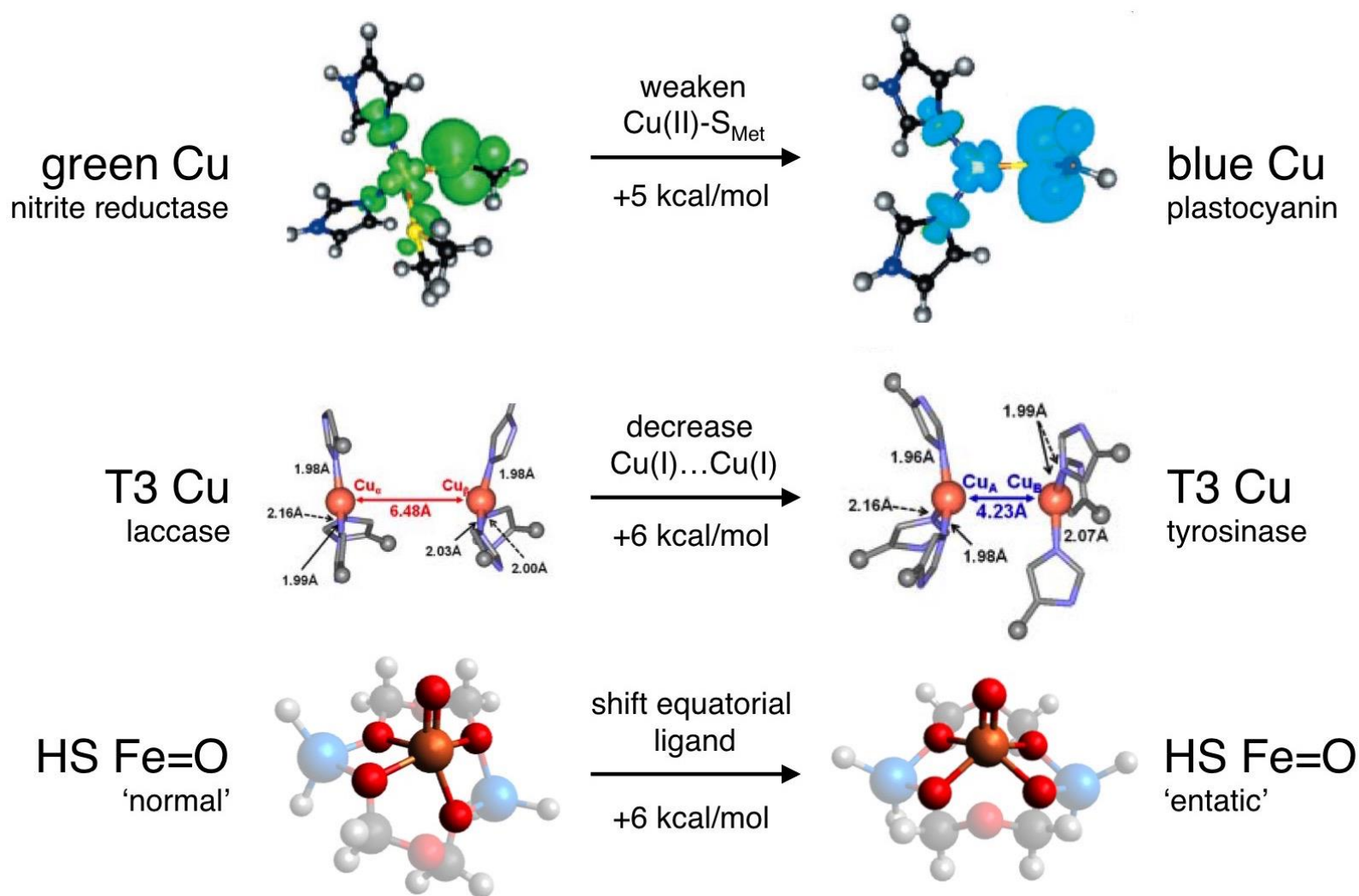

Figure 66. Comparison of 'normal' (left) and 'entatic' (right) metal active sites. Each 'normal' site undergoes a 5-6 kcal distortion that primes the corresponding 'entatic' site for function.

methionine that tunes down the reduction potential of the site to match biological electron transfer partners. Comparison to the analogous green $\mathrm{Cu}$ site (figure 66, top left), which has a less constrained $\mathrm{Cu}(\mathrm{II})-\mathrm{S}_{\mathrm{Met}}$ bond, showed constraints from the protein backbone keep the weak $\mathrm{Cu}(\mathrm{II})$-S $\mathrm{Met}$ bond in the blue $\mathrm{Cu}$ site intact by opposing the entropic contribution to the Gibbs free energy for Met dissociation. ${ }^{121}$ Protein constraints play a similar role in enforcing the weak axial Fe-SMet bond in cytochrome $c$, keeping the axial Met bound to the iron center under physiological conditions. ${ }^{237}$ The entatic stabilization of the Fe-SMet bond has been directly quantified at $\sim 4 \mathrm{kcal} / \mathrm{mol}$, and this prevents ligation by a nearby His residue or water, which would lower the reduction

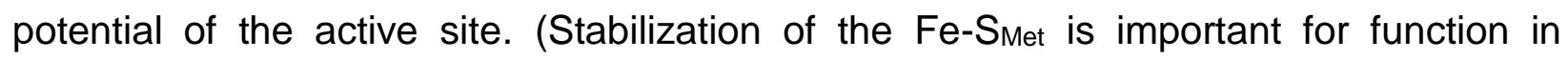
electron transfer, however the bond remains weak enough to be broken, which activates 
cyt $c$ for its role in initiating apoptosis). ${ }^{237}$ Protein constraints also play an important role in tuning the $\mathrm{O}_{2}$ binding affinity of the binuclear type $3 \mathrm{Cu}$ active sites in hemocyanin and the multi-copper oxidases (figure 66, middle). ${ }^{238}$ In hemocyanin (figure 66, middle right), the active site enforces a short $\mathrm{Cu}(\mathrm{I}) \ldots \mathrm{Cu}(\mathrm{I})$ separation of $\sim 4 \AA$ and this allows for high-affinity $\mathrm{O}_{2}$ binding. In the multi-copper oxidases (figure 66, middle left), the type 3 Cu site has a longer $\mathrm{Cu}(\mathrm{I}) \ldots \mathrm{Cu}(\mathrm{I})$ separation of $\sim 6 \AA$, and this causes $\mathrm{O}_{2}$ binding to be thermodynamically disfavored. ${ }^{238}$ (The type 3 site of the MCOs is part of a trinuclear cluster that only reacts with $\mathrm{O}_{2}$ with the participation of the $3^{\text {rd }}$ metal center.) Relative to the $2 \mathrm{Cu}(\mathrm{I})$ site in the MCOs, protein constraints in hemocyanin destabilize the $2 \mathrm{Cu}(\mathrm{I})$ site by $\sim 6 \mathrm{kcal} / \mathrm{mol}$ due to increased electrostatic repulsion, and this increases the driving force for $\mathrm{O}_{2}$ binding. ${ }^{238}$ Thus entatic contributions of $\sim 5 \mathrm{kcal} / \mathrm{mol}$ play an important role in activating a range of metalloenzyme active sites for function.

Similar concepts emerge for the $\alpha-\mathrm{O}$ site in Fe-zeolites and its activation for rapid $\mathrm{H}$-atom transfer (see section 6.3). ${ }^{35} \alpha-\mathrm{O}$ is a mononuclear, high spin $\mathrm{Fe}(\mathrm{IV})=\mathrm{O}$ site with square pyramidal geometry, where the oxo ligand bound in the axial position (as in figure 66 , bottom right). This geometry coupled to a high spin $\mathrm{d}^{4}$ electronic structure activates $\alpha-\mathrm{O}$ for rapid $\mathrm{H}$-atom abstraction from strong $\mathrm{C}-\mathrm{H}$ bonds (e.g. methane). The high spin state results in occupation of the $d_{x^{2}-y^{2}}$ orbital, which is strongly antibonding with respect to the equatorial ligands. The weak interaction with the equatorial ligands coupled to a vacant trans axial position is compensated for by strong donation of the oxo, resulting in an unusually covalent $\mathrm{Fe}=\mathrm{O}$ bond that efficiently polarizes toward an ferric-oxyl electronic structure with minimal Fe-O elongation. This results in high oxo $2 p$ content in the reactive molecular orbitals, making $\alpha-\mathrm{O}$ intrinsically activated for $\mathrm{H}$-atom 
transfer. There is also an unusually strong $\mathrm{O}-\mathrm{H}$ bond in the $\mathrm{Fe}(\mathrm{III})-\mathrm{OH}$ first product (over $100 \mathrm{kcal} / \mathrm{mol}$ ), and this results in a large driving force for of $\mathrm{H}$-atom transfer associated with axial square pyramidal geometry.

The geometric/electronic structure of $\alpha-\mathrm{O}$ that leads to high reactivity is inherently unstable, as a high spin $d^{4}$ square pyramidal site with a strong axial ligand will experience a distorting force to shift an equatorial ligand to fill the vacant trans axial position (see figure 5.6 and figure 66 , bottom left). ${ }^{35,197}$ This distortion would result in stronger bonding to the zeolite lattice, stabilizing the site by $6 \mathrm{kcal} / \mathrm{mol}$. Placing a ligand trans to the oxo would result in a weaker $\mathrm{Fe}=\mathrm{O}$ bond, and thus a deactivated electronic structure, as well as a decreased driving force for $\mathrm{O}-\mathrm{H}$ bond formation. The zeolite lattice therefore activates $\alpha-O$ by providing enough distortion energy to enforce a high spin square pyramidal site with an axial oxo - similar in magnitude to the entatic effects quantified for metalloenzyme active sites (vide supra). 
7 - Concluding Comments. In this review, we have discussed the current state of the art in selective hydrocarbon oxidation by metallozeolites. These catalysts carry out remarkable chemistry, activating $\mathrm{O}_{2}\left(\right.$ and $\left.\mathrm{N}_{2} \mathrm{O}\right)$ to form intermediates that selectively hydroxylate the highly inert $\mathrm{C}-\mathrm{H}$ bonds of methane and benzene under mild conditions. Copper and iron active sites have taken a central role in this review, as these are associated with the highest activity in selective hydrocarbon oxidation. We note that $\mathrm{Cu}$ - and Fe-zeolites perform a number of other interesting reactions not discussed here, including selective catalytic reduction of $\mathrm{NO}_{x}$ pollutants. ${ }^{239}$ Zeolites containing other metals are also known to activate methane, but at higher temperatures and with diminished selectivity for methanol. Studies discussed in this review demonstrate that molecular-level insight from site selective spectroscopy is vital for understanding the geometric and electronic structure, and therefore reactivity of metal active sites in zeolites. While we have focused on the coupling spectroscopic data to initial steps in $\mathrm{H}$ atom abstraction and electrophilic aromatic hydroxylation, we anticipate spectroscopy will soon elucidate subsequent steps in these reaction coordinates (i.e. C-O bond formation in methanol and phenol synthesis). These relate directly to issues with catalyst performance, such as deactivation through product trapping.

Advances in spectroscopic methodology discussed in this review have elevated the insight available for heterogeneous active sites to the level achieved in bioinorganic chemistry. $\mathrm{Cu}$ - and Fe-zeolites (Cu-ZSM-5/MOR and Fe-BEA, in particular) are now among the most thoroughly characterized systems that activate methane and benzene. This has enabled direct comparison to biology, where iron and copper active sites are also used to activate methane and aromatic compounds (figure 67). While there are 
significant differences between metallozeolites and metalloenzymes - in particular, that $\alpha-O$ is mononuclear while intermediate $Q$ in sMMO is binuclear - we have also highlighted three parallels between heterogeneous and biological chemistry: 1) the tuning of metal-oxygen covalency to access highly reactive intermediates; 2) second sphere effects on catalysis; and 3) entatic effects leading to active site geometric and electronic structures that are associated with high reactivity. It will be interesting to see how these features can be used to tune the reactivity of metallozeolite catalysts.

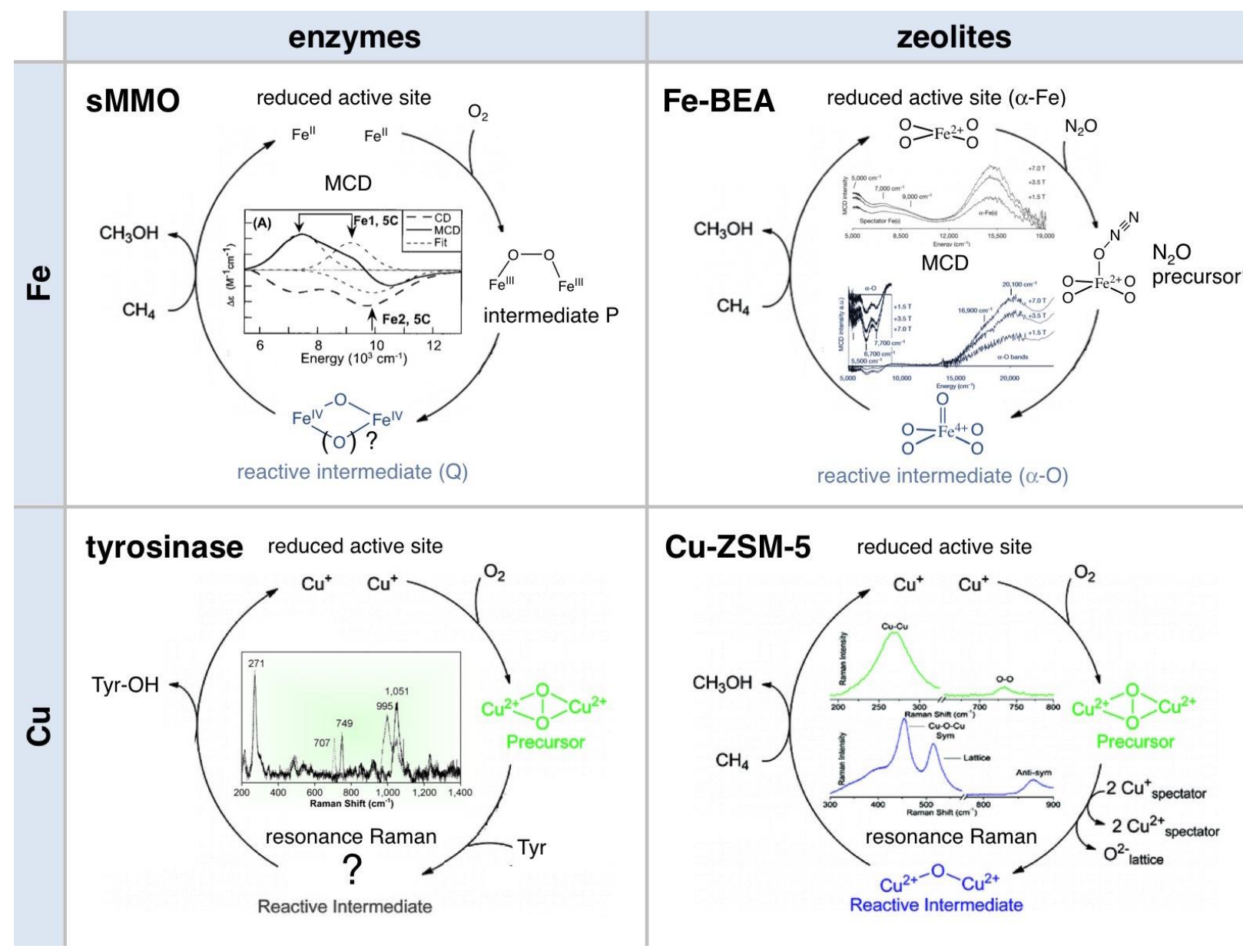

Figure 67. Comparison of $\mathrm{Fe}$ and $\mathrm{Cu}$ enzymes and zeolites that hydroxylate strong $\mathrm{C}-\mathrm{H}$ bonds at low temperature, including their active site structures and catalytic intermediates defined from spectroscopy. 


\section{Author Information}

\section{Corresponding Authors}

*E-mail: edward.solomon@stanford.edu, bert.sels@biw.kuleuven.be, robert.schoonheydt@biw.kuleuven.be

\section{Notes}

The authors declare no competing financial interests.

Acknowledgements. B.E.R.S. acknowledges support from the National Science Foundation Graduate Research Fellowship Program under grant DGE-11474, and from the Munger, Pollock, Reynolds, Robinson, Smith \& Yoedicke Stanford Graduate Fellowship. Funding for this work was provided by the National Science Foundation (grant CHE-1660611 to E.I.S.), and Research Foundation-Flanders (FWO grant G0A2216N to B.F.S.). 


$\begin{array}{ll}\text { Abbreviations } & \\ \text { Abs } & \text { absorption spectroscopy } \\ \text { BAS } & \text { Brönsted acid sites } \\ \text { CASPT } & \text { Complete active space perturbation theory } \\ \text { CD } & \text { circular dichroism } \\ \text { CEC } & \text { cation exchange capacity } \\ \text { CHA } & \text { chabazite } \\ \text { DFT } & \text { density functional theory } \\ \text { DR } & \text { diffuse reflectance } \\ \text { ENDOR } & \text { electron nuclear double resonance } \\ \text { EPR } & \text { electron paramagnetic resonance } \\ \text { ESEEM } & \text { electron spin echo envelope modulation } \\ \text { FAU } & \text { faujasite } \\ \text { FER } & \text { ferrierite } \\ \text { HAA } & \text { H-atom abstraction } \\ \text { HmaS } & \text { hydroxymandelate synthase } \\ \text { HPPD } & \text { hydroxyphenylpyruvate dioxygenase } \\ \text { KIE } & \text { kinetic isotope effect } \\ \text { LF } & \text { ligand field } \\ \text { LMCT } & \text { ligand to metal charge transfer } \\ \text { LTA } & \text { Linde type A zeolite } \\ \text { MCD } & \text { magnetic circular dichroism } \\ \text { MFI } & \text { mordenite framework, inverted } \\ \text { MOR } & \text { mordenite } \\ \text { MR } & \text { membered ring } \\ \text { NIH } & \text { National Institutes of Health, named after the place where the NIH- } \\ \text { NIR } & \text { shift was first observed } \\ \text { NRVS } & \text { near ifrared } \\ \text { pMMO } & \text { nuclear resonance vibrational spectroscopy } \\ \text { rR } & \text { particulate methane monooxygenase } \\ \text { SMMO } & \text { resonance Raman spectroscopy } \\ \text { SOC } & \text { soluble methane monooxygenase } \\ \text { TEM } & \text { spin-orbit coupling } \\ \text { TMI } & \text { transmission electron microscopy } \\ \text { UV } & \text { transition metal ion } \\ \text { Vis } & \text { ultraviolet } \\ \text { VTVH } & \text { visible } \\ \text { XAS } & \text { variable-temperature variable-field } \\ \text { XPS } & \text { X-ray absorption spectroscopy } \\ \text { XANES } & \text { X-ray photoelectron spectroscopy } \\ \text { EXAFS } & \text { X-ray absorption near edge spectroscopy } \\ \text { ZFS } & \text { extended X-ray absorption fine structure } \\ \text { ZSM-5 } & \text { zocony Mobil-5 } \\ & \end{array}$




\section{References}

(1) Berkowitz, J.; Greene, J. P.; Cho, H.; Ruščić, B. Photoionization Mass Spectrometric Studies of SiHn (n=1-4). J. Chem. Phys. 1987, 86, 1235-1248.

(2) Brocławik, E. Density Functional Theory in Catalysis: Activation and Reactivity of a Hydrocarbon Molecule on a Metallic Active Site. Adv. Quantum Chem. 1998, 33, 349-367.

(3) Olivos-Suarez, A. I.; Szécsényi, À.; Hensen, E. J. M.; Ruiz-Martinez, J.; Pidko, E. A.; Gascon, J. Strategies for the Direct Catalytic Valorization of Methane Using Heterogeneous Catalysis: Challenges and Opportunities. ACS Catal. 2016, 6, 2965-2981.

(4) Periana, R. A.; Taube, D. J.; Evitt, E. R.; Löffler, D. G.; Wentrcek, P. R.; Voss, G.; Masuda, T. A Novel, High Yield System for the Oxidation of Methane to Methanol. Stud. Surf. Sci. Catal. 1994, 81, 533-544.

(5) Periana, R. A.; Bhalla, G.; Tenn, W. J.; Young, K. J. H.; Liu, X. Y.; Mironov, O.; Jones, C.; Ziatdinov, V. R. Perspectives on Some Challenges and Approaches for Developing the next Generation of Selective, Low Temperature, Oxidation Catalysts for Alkane Hydroxylation Based on the $\mathrm{CH}$ Activation Reaction. J. Mol. Catal. A Chem. 2004, 220, 7-25.

(6) Shilov, A. E.; Shul'pin, G. B. Activation of C-H Bonds by Metal Complexes. Chem. Rev. 1997, 97, 2879-2932.

(7) Pérez, P. Alkane C-H Activation by Single-Site Metal Catalysis; Pérez, P. J., Ed.; Catalysis by Metal Complexes; Springer Netherlands: Dordrecht, 2012; Vol. 38.

(8) Periana, R. A. Platinum Catalysts for the High-Yield Oxidation of Methane to a 
Methanol Derivative. Science 1998, 280, 560-564.

(9) Stahl, S. S.; Labinger, J. A.; Bercaw, J. E. Homogeneous Oxidation of Alkanes by Electrophilic Late Transition Metals. Angew. Chemie Int. Ed. 1998, 37, 21802192.

(10) Hammond, C.; Conrad, S.; Hermans, I. Oxidative Methane Upgrading. ChemSusChem 2012, 5, 1668-1686.

(11) Olah, G. A.; Goeppert, A.; Prakash, G. K. S. Beyond Oil and Gas : The Methanol Economy; Wiley-VCH, 2009.

(12) Horn, R.; Schlögl, R. Methane Activation by Heterogeneous Catalysis. Catal. Lett. 2015, 145, 23-39.

(13) Khan, M. S.; Park, J. H.; Chaniago, Y. D.; Lee, M. Energy Efficient Process Structure Design of LNG/NGL Recovery for Offshore FLNG Plant. Energy Procedia 2014, 61, 599-602.

(14) Gao, J.; Zheng, Y.; Jehng, J.-M.; Tang, Y.; Wachs, I. E.; Podkolzin, S. G. Identification of Molybdenum Oxide Nanostructures on Zeolites for Natural Gas Conversion. Science 2015, 348, 686-690.

(15) Liptáková, B.; Báhidský, M.; Hronec, M. Preparation of Phenol from Benzene by One-Step Reaction. Appl. Catal. A Gen. 2004, 263, 33-38.

(16) Stahl, S. S.; Alsters, P. L. Liquid Phase Aerobic Oxidation Catalysis : Industrial Applications and Academic Perspectives; Wiley-VCH, 2016.

(17) Labinger, J. A.; Bercaw, J. E. Understanding and Exploiting C-H Bond Activation. Nature 2002, 417, 507-514.

(18) Solomon, E. I.; Heppner, D. E.; Johnston, E. M.; Ginsbach, J. W.; Cirera, J.; 
Qayyum, M.; Kieber-Emmons, M. T.; Kjaergaard, C. H.; Hadt, R. G.; Tian, L. Copper Active Sites in Biology. Chemical Reviews. 2014, pp 3659-3853.

(19) Solomon, E. I.; Brunold, T. C.; Davis, M. I.; Kemsley, J. N.; Lee, S.-K.; Lehnert, N.; Neese, F.; Skulan, A. J.; Yang, Y.-S.; Zhou, J. Geometric and Electronic Structure/function Correlations in Non-Heme Iron Enzymes. Chem. Rev. 2000, 100, 235-350.

(20) Guroff, G.; Renson, J.; Udenfriend, S.; Daly, J. W.; Jerina, D. M.; Witkop, B. Hydroxylation-Induced Migration: The NIH Shift. Science 1967, 157, 1524-1530.

(21) Ross, M. O.; Rosenzweig, A. C. A Tale of Two Methane Monooxygenases. J. Biol. Inorg. Chem. 2017, 22, 307-319.

(22) Hagen, J. Industrial Catalysis: A Practical Approach, 2nd ed.; Wiley-VCH Verlag GmbH \& Co. KGaA: Weinheim, FRG, 2006.

(23) Soussan, L.; Pen, N.; Belleville, M. P.; Marcano, J. S.; Paolucci-Jeanjean, D. Alkane Biohydroxylation: Interests, Constraints and Future Developments. Journal of Biotechnology. 2016, pp 117-142.

(24) Gunsalus, N. J.; Koppaka, A.; Park, S. H.; Bischof, S. M.; Hashiguchi, B. G.; Periana, R. A. Homogeneous Functionalization of Methane. Chem. Rev. 2017, 117, 8521-8573.

(25) Erb, T. J.; Jones, P. R.; Bar-Even, A. Synthetic Metabolism: Metabolic Engineering Meets Enzyme Design. Curr. Opin. Chem. Biol. 2017, 37, 56-62.

(26) Xiao, D. J.; Bloch, E. D.; Mason, J. A.; Queen, W. L.; Hudson, M. R.; Planas, N.; Borycz, J.; Dzubak, A. L.; Verma, P.; Lee, K.; et al. Oxidation of Ethane to Ethanol by N2O in a Metal-organic Framework with Coordinatively Unsaturated iron(II) 
Sites. Nat. Chem. 2014, 6, 590-595.

(27) Li, Z.; Peters, A. W.; Bernales, V.; Ortuño, M. A.; Schweitzer, N. M.; DeStefano, M. R.; Gallington, L. C.; Platero-Prats, A. E.; Chapman, K. W.; Cramer, C. J.; et al. Metal-Organic Framework Supported Cobalt Catalysts for the Oxidative Dehydrogenation of Propane at Low Temperature. ACS Cent. Sci. 2017, 3, 3138.

(28) Herron, N. Zeolite Catalysts as Enzyme Mimics. Toward Silicon-Based Life? Biocatal. Biomimetics 1989, 141-154.

(29) Xuereb, D. J.; Raja, R. Design Strategies for Engineering Selectivity in BioInspired Heterogeneous Catalysts. Catal. Sci. Technol. 2011, 1, 517-534.

(30) Xuereb, D. J.; Dzierzak, J.; Raja, R. From Zeozymes to Bio-Inspired Heterogeneous Solids: Evolution of Design Strategies for Sustainable Catalysis. Catal. Today 2012, 198, 19-34.

(31) Srinivas, D.; Sivasanker, S. Ã. Biomimetic Oxidations Using Transition Metal Complexes Encapsulated in Zeolites. 2003, 7, 121-132.

(32) Panov, G. I.; Sobolev, V. I.; Kharitonov, A. S. The Role of Iron in N2O Decomposition on ZSM-5 Zeolite and Reactivity of the Surface Oxygen Formed. J. Mol. Catal. 1990, 61, 85-97.

(33) Dubkov, K. A.; Sobolev, V. I.; Talsi, E. P.; Rodkin, M. A.; Watkins, N. H.; Shteinman, A. A.; Panov, G. I. Kinetic Isotope Effects and Mechanism of Biomimetic Oxidation of Methane and Benzene on FeZSM-5 Zeolite. J. Mol. Catal. A Chem. 1997, 123, 155-161.

(34) Notté, P. P. The AlphOxTM Process or the One- step Hydroxylation of Benzene 
into Phenol by Nitrous Oxide. Understanding and Tuning the ZSM- 5 Catalyst Activities. Top. Catal. 2000, 13, 387-394.

(35) Snyder, B. E. R.; Vanelderen, P.; Bols, M. L.; Hallaert, S. D.; Böttger, L. H.; Ungur, L.; Pierloot, K.; Schoonheydt, R. A.; Sels, B. F.; Solomon, E. I. The Active Site of Low-Temperature Methane Hydroxylation in Iron-Containing Zeolites. Nature 2016, 536, 317-321.

(36) Groothaert, M. H.; Smeets, P. J.; Sels, B. F.; Jacobs, P. A.; Schoonheydt, R. A. Selective Oxidation of Methane by the Bis $(\mu$-Oxo)dicopper Core Stabilized on ZSM-5 and Mordenite Zeolites. J. Am. Chem. Soc. 2005, 127, 1394-1395.

(37) Woertink, J. S.; Smeets, P. J.; Groothaert, M. H.; Vance, M. A.; Sels, B. F.; Schoonheydt, R. A.; Solomon, E. I. A [Cu2O]2+ Core in Cu-ZSM-5, the Active Site in the Oxidation of Methane to Methanol. Proc. Natl. Acad. Sci. 2009, 106, 18908-18913.

(38) International Zeolite Association. IZA Structure database http://europe.izastructure.org/IZA-SC/ftc_table.php (accessed Apr 25, 2017).

(39) Gounaris, C. E.; First, E. L.; Wei, J.; Floudas, C. A.; Ranjan, R.; Tsapatsis, M. ZEOMICS: Zeolites and Microporous Structures Characterization http://helios.princeton.edu/zeomics/ (accessed May 6, 2017).

(40) Dědeček, J.; Sobalík, Z.; Wichterlová, B. Siting and Distribution of Framework Aluminium Atoms in Silicon-Rich Zeolites and Impact on Catalysis. Catal. Rev. 2012, 54, 135-223.

(41) Gábová, V.; Dědeček, J.; Čejka, J. Control of Al Distribution in ZSM-5 by Conditions of Zeolite Synthesis. Chem. Commun. 2003, No. 10, 1196-1197. 
(42) Di lorio, J. R.; Gounder, R. Controlling the Isolation and Pairing of Aluminum in Chabazite Zeolites Using Mixtures of Organic and Inorganic Structure-Directing Agents. Chem. Mater. 2016, 28, 2236-2247.

(43) Van Bokhoven, J. A.; Lee, T.-L.; Drakopoulos, M.; Lamberti, C.; Thieß, S.;

Zegenhagen, J. Determining the Aluminium Occupancy on the Active T-Sites in Zeolites Using X-Ray Standing Waves. Nat. Mater. 2008, 7, 551-555.

(44) Mccusker, L. B.; Von Dreele, R. B.; Cox, D. E.; Louër, D.; Scardi, P. Rietveld Refnement Guidelines. J. Appl. Crystallogr. 1999, 32, 36-50.

(45) Rietveld, H. M. A Profile Refinement Method for Nuclear and Magnetic Structures. J. Appl. Crystallogr. 1969, 2, 65-71.

(46) Papoular, R. J.; Cox, D. E. Model-Free Search for Extra-Framework Cations in Zeolites Using Powder Diffraction. Europhys. Lett. 1995, 32, 337-342.

(47) Dalconi, M. C.; Cruciani, G.; Alberti, A.; Ciambelli, P. Co- and Ni-Exchanged Ferrierite: The Contribution of Synchrotron X-Ray Diffraction Data to Siting of TMls. Catal. Today 2005, 110, 345-350.

(48) Barrer, R. M. Zeolites and Clay Minerals as Sorbents and Molecular Sieves; Academic Press: London, 1978.

(49) Mortier, W.; Structure Commission of the International Zeolite Association. Compilation of Extra Framework Sites in Zeolites; Butterworth Scientific Limited: Leuven, 1982.

(50) Esemann, H.; Förster, H.; Geidel, E.; Krause, K. Exploring Cation Siting in Zeolite ZSM-5 by Infrared Spectroscopy, EXAFS and Computer Simulations. Microporous Mater. 1996, 6, 321-329. 
(51) Dalconi, M. C.; Alberti, A.; Cruciani, G.; Ciambelli, P.; Fonda, E. Siting and Coordination of Cobalt in Ferrierite: XRD and EXAFS Studies at Different Co Loadings. Microporous Mesoporous Mater. 2003, 62, 191-200.

(52) Benco, L.; Bucko, T.; Hafner, J.; Toulhoat, H. Periodic DFT Calculations of the Stability of $\mathrm{Al} / \mathrm{Si}$ Substitutions and Extraframework $\mathrm{Zn}{ }^{2+}$ Cations in Mordenite and Reaction Pathway for the Dissociation of $\mathrm{H} 2$ and CH4. J. Phys. Chem. B 2005, 109, 20361-20369.

(53) Dědeček, J.; Kaucký, D.; Wichterlová, B. Co2+ Ion Siting in Pentasil-Containing Zeolites, Part 3.: Co2+ Ion Sites and Their Occupation in ZSM-5: A VIS Diffuse Reflectance Spectroscopy Study. Microporous Mesoporous Mater. 2000, 35, 483-494.

(54) Palagin, D.; Knorpp, A. J.; Pinar, A. B.; Ranocchiari, M.; van Bokhoven, J. A. Assessing the Relative Stability of Copper Oxide Clusters as Active Sites of a CuMOR Zeolite for Methane to Methanol Conversion: Size Matters? Nanoscale 2017, 9, 1144-1153.

(55) Grundner, S.; Markovits, M. A. C.; Li, G.; Tromp, M.; Pidko, E. A.; Hensen, E. J. M.; Jentys, A.; Sanchez-Sanchez, M.; Lercher, J. A. Single-Site Trinuclear Copper Oxygen Clusters in Mordenite for Selective Conversion of Methane to Methanol. Nat. Commun. 2015, 6, 7546.

(56) Battiston, A. A.; Bitter, J. H.; Koningsberger, D. C. Reactivity of Binuclear Fe Complexes in over-Exchanged Fe/ZSM5, Studied by in Situ XAFS Spectroscopy 2. Selective Catalytic Reduction of NO with Isobutane. J. Catal. 2003, 218, 163177. 
(57) Dubkov, K. A.; Ovanesyan, N. S.; Shteinman, A. A.; Starokon, E. V.; Panov, G. I. Evolution of Iron States and Formation of $\alpha$-Sites upon Activation of FeZSM-5 Zeolites. J. Catal. 2002, 207, 341-352.

(58) Jiša, K.; Nováková, J.; Schwarze, M.; Vondrová, A.; Sklenák, S.; Sobalik, Z. Role of the Fe-Zeolite Structure and Iron State in the N2O Decomposition: Comparison of Fe-FER, Fe-BEA, and Fe-MFI Catalysts. J. Catal. 2009, 262, 27-34.

(59) Iwamoto, M.; Yahiro, H.; Tanda, K.; Mizuno, N.; Mine, Y.; Kagawa, S. Removal of Nitrogen Monoxide through a Novel Catalytic Process. 1. Decomposition on Excessively Copper-Ion-Exchanged ZSM-5 Zeolites. J. Phys. Chem. 1991, 95, 3727-3730.

(60) Occhiuzzi, M.; Fierro, G.; Ferraris, G.; Moretti, G. Unusual Complete Reduction of Cu2+ Species in Cu-ZSM-5 Zeolites under Vacuum Treatment at High Temperature. Chem. Mater. 2012, 24, 2022-2031.

(61) Larsen, S. C.; Aylor, A.; Bell, A. T.; Reimer, J. A. Electron Paramagnetic Resonance Studies of Copper lon-Exchanged ZSM-5. J. Phys. Chem. 1994, 98, 11533-11540.

(62) Jacobs, P. A.; Beyer, H. K. Evidence for the Nature of True Lewis Sites in Faujasite-Type Zeolites. J. Phys. Chem. 1979, 83, 1174-1177.

(63) Vanelderen, P.; Vancauwenbergh, J.; Tsai, M.-L.; Hadt, R. G.; Solomon, E. I.; Schoonheydt, R. A.; Sels, B. F. Spectroscopy and Redox Chemistry of Copper in Mordenite. ChemPhysChem 2015, 15, 91-99.

(64) Moretti, G.; Ferraris, G.; Fierro, G.; Jacono, M. Lo; Morpurgo, S.; Faticanti, M. Dimeric $\mathrm{Cu}(\mathrm{I})$ Species in Cu-ZSM-5 Catalysts: The Active Sites for the NO 
Decomposition. J. Catal. 2005, 232, 476-487.

(65) Groothaert, M. H.; Lievens, K.; Leeman, H.; Weckhuysen, B. M.; Schoonheydt, R. A. An Operando Optical Fiber UV-vis Spectroscopic Study of the Catalytic Decomposition of NO and N2O over Cu-ZSM-5. J. Catal. 2003, 220, 500-512.

(66) Smeets, P. J.; Groothaert, M. H.; Schoonheydt, R. A. Cu Based Zeolites: A UVvis Study of the Active Site in the Selective Methane Oxidation at Low Temperatures. Catal. Today 2005, 110, 303-309.

(67) Markovits, M. A. C.; Jentys, A.; Tromp, M.; Sanchez-Sanchez, M.; Lercher, J. A. Effect of Location and Distribution of AI Sites in ZSM-5 on the Formation of CuOxo Clusters Active for Direct Conversion of Methane to Methanol. Top. Catal. 2016, 59, 1554-1563.

(68) Dědeček, J.; Kaucký, D.; Wichterlová, B.; Gonsiorová, O. Co(II) lons as Probes of Al Distribution in the Framework of Zeolites. ZSM-5 Study. Phys. Chem. Chem. Phys. 2002, 4, 5406-5413.

(69) Sazama, P.; Dědeček, J.; Gábová, V.; Wichterlová, B.; Spoto, G.; Bordiga, S. Effect of Aluminium Distribution in the Framework of ZSM-5 on Hydrocarbon Transformation. Cracking of 1-Butene. J. Catal. 2008, 254, 180-189.

(70) Gao, F.; Walter, E. D.; Kollar, M.; Wang, Y.; Szanyi, J.; Peden, C. H. F. Understanding Ammonia Selective Catalytic Reduction Kinetics over Cu/SSZ-13 from Motion of the Cu lons. J. Catal. 2014, 319, 1-14.

(71) Paolucci, C.; Parekh, A. A.; Khurana, I.; Di lorio, J. R.; Li, H.; Albarracin Caballero, J. D.; Shih, A. J.; Anggara, T.; Delgass, W. N.; Miller, J. T.; et al. Catalysis in a Cage: Condition-Dependent Speciation and Dynamics of 
Exchanged Cu Cations in SSZ-13 Zeolites. J. Am. Chem. Soc. 2016, 138, 60286048.

(72) Borfecchia, E.; Lomachenko, K. A.; Giordanino, F.; Falsig, H.; Beato, P.; Soldatov, A. V.; Bordiga, S.; Lamberti, C. Revisiting the Nature of Cu Sites in the Activated Cu-SSZ-13 Catalyst for SCR Reaction. Chem. Sci. 2015, 6, 548-563.

(73) Zhao, Z.-J.; Kulkarni, A.; Vilella, L.; Nørskov, J. K.; Studt, F. Theoretical Insights into the Selective Oxidation of Methane to Methanol in Copper-Exchanged Mordenite. ACS Catal. 2016, 6, 3760-3766.

(74) Kulkarni, A. R.; Zhao, Z.-J.; Siahrostami, S.; Nørskov, J. K.; Studt, F. Monocopper Active Site for Partial Methane Oxidation in Cu-Exchanged 8MR Zeolites. ACS Catal. 2016, 6, 6531-6536.

(75) Kwak, J. H.; Varga, T.; Peden, C. H. F.; Gao, F.; Hanson, J. C.; Szanyi, J. Following the Movement of Cu lons in a SSZ-13 Zeolite during Dehydration, Reduction and Adsorption: A Combined in Situ TP-XRD, XANES/DRIFTS Study. J. Catal. 2014, 314, 83-93.

(76) Giordanino, F.; Vennestrøm, P. N. R.; Lundegaard, L. F.; Stappen, F. N.; Mossin, S.; Beato, P.; Bordiga, S.; Lamberti, C. Characterization of Cu-Exchanged SSZ13: A Comparative FTIR, UV-Vis, and EPR Study with Cu-ZSM-5 and Cu-\$B\$ with Similar Si/Al and Cu/AI Ratios. Dalt. Trans. 2013, 42, 12741-12761.

(77) Grundner, S.; Luo, W.; Sanchez-Sanchez, M.; Lercher, J. Synthesis of Single-Site Copper Catalysts for Methane Partial Oxidation. Chem. Commun. 2015, 52, 1-17.

(78) Narsimhan, K.; Michaelis, V. K.; Mathies, G.; Gunther, W. R.; Griffin, R. G.; Román-Leshkov, Y. Methane to Acetic Acid over Cu-Exchanged Zeolites: 
Mechanistic Insights from a Site-Specific Carbonylation Reaction. J. Am. Chem. Soc. 2015, 137, 1825-1832.

(79) Li, G.; Vassilev, P.; Sanchez-Sanchez, M.; Lercher, J. A.; Hensen, E. J. M.; Pidko, E. A. Stability and Reactivity of Copper Oxo-Clusters in ZSM-5 Zeolite for Selective Methane Oxidation to Methanol. J. Catal. 2016, 338, 305-312.

(80) Veefkind, V. A.; Smidt, M. L.; Lercher, J. A. On the Role of Strength and Location of Brønsted Acid Sites for Ethylamine Synthesis on Mordenite Catalysts. Appl. Catal. A Gen. 2000, 194, 319-332.

(81) Mortier, W. J.; Schoonheydt, R. A. Surface and Solid State Chemistry of Zeolites. Prog. Solid State Chem. 1985, 16, 1-125.

(82) Baekelandt, B. G.; Mortier, W.; Schoonheydt, R. Structural Chemistry of Zeolites : Solid State Electronegativity and Sensitivity Analysis; Catlow, R. A., Ed.; Academic Press: London, 1992.

(83) De Tavernier, S. G.; Schoonheydt, R. A.; Mortier, W. J. Zeolites for the Nineties: Recent Progress Reports on the 8th Int. Zeolite Conf.; Elsevier,: Amsterdam ;, 1989.

(84) Wulfers, M. J.; Teketel, S.; Ipek, B.; Lobo, R. F. Conversion of Methane to Methanol on Copper-Containing Small-Pore Zeolites and Zeotypes. Chem. Commun. 2015, 51, 4447-4450.

(85) Zecchina, A.; Rivallan, M.; Berlier, G.; Lamberti, C.; Ricchiardi, G. Structure and Nuclearity of Active Sites in Fe-Zeolites: Comparison with Iron Sites in Enzymes and Homogeneous Catalysts. Phys. Chem. Chem. Phys. 2007, 9, 3483-3499.

(86) Mauvezin, M.; Delahay, G.; Coq, B.; Kieger, S.; Jumas, J. C.; Olivier-Fourcade, J. 
Identification of Iron Species in Fe-BEA: Influence of the Exchange Level. J. Phys. Chem. B 2001, 105, 928-935.

(87) Lobree, L. J.; Hwang, I.-C.; Reimer, J. A.; Bell, A. T. Investigations of the State of Fe in H-ZSM-5. J. Catal. 1999, 186, 242-253.

(88) Panov, G. I.; Uriarte, A. K.; Rodkin, M. A.; Sobolev, V. I. Generation of Active Oxygen Species on Solid Surfaces. Opportunity for Novel Oxidation Technologies over Zeolites. Catal. Today 1998, 41, 365-385.

(89) Marturano, P.; Drozdová, L.; Pirngruber, G. D.; Kogelbauer, A.; Prins, R. The Mechanism of Formation of the Fe Species in Fe/ZSM-5 Prepared by CVD. Phys. Chem. Chem. Phys. 2001, 3, 5585-5595.

(90) Chen, H.-Y.; Sachtler, W. M. . Activity and Durability of Fe/ZSM-5 Catalysts for Lean Burn NOx Reduction in the Presence of Water Vapor. Catal. Today 1998, 42, 73-83.

(91) Voskoboinikov, T. V; Chen, H.-Y.; Sachtler, W. M. . On the Nature of Active Sites in Fe/ZSM-5 Catalysts for NOx Abatement. Appl. Catal. B Environ. 1998, 19, 279287.

(92) Hammond, C.; Forde, M. M.; Ab Rahim, M. H.; Thetford, A.; He, Q.; Jenkins, R. L.; Dimitratos, N.; Lopez-Sanchez, J. A.; Dummer, N. F.; Murphy, D. M.; et al. Direct Catalytic Conversion of Methane to Methanol in an Aqueous Medium by Using Copper-Promoted Fe-ZSM-5. Angew. Chemie Int. Ed. 2012, 51, 51295133.

(93) Hammond, C.; Hermans, I.; Dimitratos, N. Biomimetic Oxidation with Fe-ZSM-5 and $\mathrm{H}_{2} \mathrm{O} 2$ ? Identification of an Active, Extra-Framework Binuclear Core and an 
$\mathrm{Fe}(\mathrm{III}) \mathrm{OOH}$ Intermediate with Resonance-Enhanced Raman Spectroscopy.

ChemCatChem 2015, 7, 434-440.

(94) Xia, H.; Sun, K.; Sun, K.; Feng, Z.; Li, W. X.; Li, C. Direct Spectroscopic

Observation of $\mathrm{Fe}(\mathrm{III})$-Phenolate Complex Formed From the Reaction of Benzene

With Peroxide Species on Fe/ZSM-5 At Room Temperature. J. Phys. Chem. C

2008, 112, 9001-9005.

(95) Gao, Z.-X.; Kim, H.-S.; Sun, Q.; Stair, P. C.; Sachtler, W. M. H. UV- Raman

Characterization of Iron Peroxo Adsorbates on Fe/MFI Catalyst with High Activity

for NO X Reduction. J. Phys. Chem. B 2001, 105, 6186-6190.

(96) Klier, K.; Hutta, P. J.; Kellerman, R. Electronic Structure and Stability of Transition Metal Ions in Zeolites. In Molecular Sieves_Il; Webster: New York, 1977; pp $108-119$.

(97) Klier, K.; Kellerman, R.; Hutta, P. J. Spectra of Synthetic Zeolites Containing Transition Metal lons. V. ${ }^{*} \Pi$ Complexes of Olefins and Acetylene with Co(II)A Molecular Sieve. J. Chem. Phys. 1974, 61, 4224-4234.

(98) Schoonheydt, R. A. Transition Metal Ions in Zeolites: Siting and Energetics of Cu2+. Catal. Rev. 1993, 35, 129-168.

(99) Delabie, A.; Pierloot, K.; Groothaert, M. H.; Weckhuysen, B. M.; Schoonheydt, R. A. The Siting of $\mathrm{Cu}(\mathrm{II})$ in Mordenite: A Theoretical Spectroscopic Study. Phys. Chem. Chem. Phys. 2002, 4, 134-145.

(100) Pierloot, K.; Delabie, A.; Groothaert, M. H.; Schoonheydt, R. A. A Reinterpretation of the EPR Spectra of $\mathrm{Cu}(\mathrm{II})$ in Zeolites A, Y and ZK4, Based on Ab Initio Cluster Model Calculations. Phys. Chem. Chem. Phys. 2001, 3, 2174-2183. 
(101) Groothaert, M. H.; Pierloot, K.; Delabie, A.; Schoonheydt, R. A. Identification of $\mathrm{Cu}$ (ii) Coordination Structures in Cu-ZSM-5, Based on a DFT/ab Initio Assignment of the EPR Spectra. Phys. Chem. Chem. Phys. 2003, 5, 2135-2144.

(102) Delabie, A.; Pierloot, K.; Groothaert, M. H.; Schoonheydt, R. A.; Vanquickenborne, L. G. The Coordination of Cull in Zeolites - Structure and Spectroscopic Properties. Eur. J. Inorg. Chem. 2002, 2002, 515-530.

(103) Mignon, P.; Pidko, E. A.; Van Santen, R. A.; Geerlings, P.; Schoonheydt, R. A. Understanding the Reactivity and Basicity of Zeolites: A Periodic DFT Study of the Disproportionation of N2O4 on Alkali-Cation-Exchanged Zeolite Y. Chem. - A Eur. J. 2008, 14, 5168-5177.

(104) Evgeny, P. A.; Mignon, P.; Geerlings, P.; Schoonheydt, R. A.; van Santen, R. A. A Periodic DFT Study of N2O4 Disproportionation on Alkali-Exchanged Zeolites X. J. Phys. Chem. C 2008, 112, 5510-5519.

(105) Schoonheydt, R. A.; Geerlings, P.; Pidko, E. A.; van Santen, R. A. The Framework Basicity of Zeolites. J. Mater. Chem. 2012, 22, 18705.

(106) Hammond, C. Intensification Studies of Heterogeneous Catalysts: Probing and Overcoming Catalyst Deactivation during Liquid Phase Operation. Green Chem. 2017, 19, 2711-2728.

(107) Solomon, E. I.; Hodgson, K. O. Spectroscopic Methods in Bioinorganic Chemistry; ACS Publications: Washington D.C., 1998.

(108) Weltner, W. Magnetic Atoms and Molecules; Courier Corporation: Mineola, NY, 1989.

(109) Kahn, O. Molecular Magnetism; VCH Publishers: New York, 1993. 
(110) Abragam, A.; Bleaney, B. Electron Paramagnetic Resonance of Transition Ions; OUP Oxford, 2012.

(111) Ballhausen, C. J.; Weiner, M. A. Introduction to Ligand Field Theory. J. Electrochem. Soc. 1963, 110, 97C-97C.

(112) Hoffman, B. M. ENDOR of Metalloenzymes. Acc. Chem. Res. 2003, 36, 522-529.

(113) Campochiaro, C.; Pavel, E. G.; Solomon, E. I. Saturation Magnetization Magnetic Circular-Dichroism Spectroscopy of Systems with Positive Zero-Field SplittingsApplication to Fesif6-Center-Dot-6H (2) O. Inorg. Chem. 1995, 34, 4669-4675.

(114) Levy, P. M. Antisymmetric Exchange. Phys. Rev. Lett. 1968, 20, 1366-1370.

(115) Bencini, A.; Gatteschi, D. Electron Paramagnetic Resonance of Exchange Coupled Systems; Springer Science \& Business Media: Mineola, NY, 2012.

(116) Solomon, E. I.; Pavel, E. G.; Loeb, K. E.; Campochiaro, C. Magnetic Circular Dichroism Spectroscopy as a Probe of the Geometric and Electronic Structure of Non-Heme Ferrous Enzymes. Coord. Chem. Rev. 1995, 144, 369-460.

(117) Solomon, E. I. Spectroscopic Methods in Bioinorganic Chemistry: Blue to Green to Red Copper Sites. Inorg. Chem. 2006, 45, 8012-8025.

(118) Vallee, B. L.; Williams, R. J. Metalloenzymes: The Entatic Nature of Their Active Sites. Proc. Natl. Acad. Sci. 1968, 59, 498-505.

(119) Solomon, E. I.; Scott, R. A.; King, R. B.; Lever, A. B. P.; Leznoff, C. C.; Schmidbauer, H.; Lee, J. D.; Moorhouse, J.; Karlin, K. D.; Zhai, T. Inorganic Electronic Structure and Spectroscopy, Volume I: Methodology; Wiley Interscience: Hoboken, 2006.

(120) Tang, J.; Albrecht, A. C. Developments in the Theories of Vibrational Raman 
Intensities. In Raman spectroscopy; Springer, 1970; pp 33-68.

(121) Ghosh, S.; Xie, X.; Dey, A.; Sun, Y.; Scholes, C. P.; Solomon, E. I.

Thermodynamic Equilibrium between Blue and Green Copper Sites and the Role of the Protein in Controlling Function. Proc. Natl. Acad. Sci. 2009, 106, 49694974.

(122) Neese, F.; Solomon, E. I. MCD C-Term Signs, Saturation Behavior, and Determination of Band Polarizations in Randomly Oriented Systems with Spin S $\geq$ 1/2. Applications to $S=1 / 2$ and $S=5 / 2$. Inorg. Chem. 1999, 38, 1847-1865.

(123) Piepho, S. B.; Schatz, P. N. Group Theory in Spectroscopy: With Applications to Magnetic Circular Dichroism; Wiley-Interscience monographs in chemical physics; Wiley: Hoboken, 1983.

(124) Schatz, P. N.; Mowery, R. L.; Krausz, E. R. MCD/MCPL Saturation Theory with Application to Molecules in Dœ H and Its Subgroups. Mol. Phys. 1978, 35, 15371557.

(125) Stephens, P. J. Magnetic Circular Dichroism. Adv. Chem. Phys. 1976, 35, 197264.

(126) Whittaker, J. W.; Solomon, E. I. Spectroscopic Studies on Ferrous Non-Heme Iron Active Sites: Magnetic Circular Dichroism of Mononuclear Fe Sites in Superoxide Dismutase and Lipoxygenase. J. Am. Chem. Soc. 1988, 110, 5329-5339.

(127) Münck, E.; Ksurerus, K.; Hendrich, M. P. [17] Combining Mössbauer Spectroscopy with Integer Spin Electron Paramagnetic Resonance. Methods Enzymol. 1993, 227, 463-479.

(128) Hendrich, M. P.; Debrunner, P. G. Integer-Spin Electron Paramagnetic 
Resonance of Iron Proteins. Biophys. J. 1989, 56, 489-506.

(129) Sturhahn, W.; Toellner, T. S.; Alp, E. E.; Zhang, X.; Ando, M.; Yoda, Y.; Kikuta, S.; Seto, M.; Kimball, C. W.; Dabrowski, B. Phonon Density of States Measured by Inelastic Nuclear Resonant Scattering. Phys. Rev. Lett. 1995, 74, 3832-3835.

(130) Scheidt, W. R.; Durbin, S. M.; Sage, J. T. Nuclear Resonance Vibrational spectroscopy-NRVS. J. Inorg. Biochem. 2005, 99, 60-71.

(131) Wong, S. D.; Srnec, M.; Matthews, M. L.; Liu, L. V; Kwak, Y.; Park, K.; Bell III, C. B.; Alp, E. E.; Zhao, J.; Yoda, Y. Elucidation of the Fe (IV)= O Intermediate in the Catalytic Cycle of the Halogenase SyrB2. Nature 2013, 499, 320-323.

(132) Wong, S. D.; Bell, C. B.; Liu, L. V; Kwak, Y.; England, J.; Alp, E. E.; Zhao, J.; Que, L.; Solomon, E. I. Nuclear Resonance Vibrational Spectroscopy on the FeIV $\square$ $\mathrm{OS}=2$ Non- Heme Site in TMG3tren: Experimentally Calibrated Insights into Reactivity. Angew. Chemie Int. Ed. 2011, 50, 3215-3218.

(133) Bell, C. B.; Wong, S. D.; Xiao, Y.; Klinker, E. J.; Tenderholt, A. L.; Smith, M. C.; Rohde, J.; Que, L.; Cramer, S. P.; Solomon, E. I. A Combined NRVS and DFT Study of FelV=O Model Complexes: A Diagnostic Method for the Elucidation of Non- Heme Iron Enzyme Intermediates. Angew. Chemie Int. Ed. 2008, 47, 90719074.

(134) Vanelderen, P.; Snyder, B. E. R.; Tsai, M.-L.; Hadt, R. G.; Vancauwenbergh, J.; Coussens, O.; Schoonheydt, R. A.; Sels, B. F.; Solomon, E. I. Spectroscopic Definition of the Copper Active Sites in Mordenite: Selective Methane Oxidation. J. Am. Chem. Soc. 2015, 137, 6383-6392.

(135) Tsai, M.-L.; Hadt, R. G.; Vanelderen, P.; Sels, B. F.; Schoonheydt, R. A.; 
Solomon, E. I. [Cu2O]2+ Active Site Formation in Cu-ZSM-5: Geometric and Electronic Structure Requirements for N2O Activation. J. Am. Chem. Soc. 2014, 136, 3522-3529.

(136) Smeets, P. J.; Hadt, R. G.; Woertink, J. S.; Vanelderen, P.; Schoonheydt, R. A.; Sels, B. F.; Solomon, E. I. Oxygen Precursor to the Reactive Intermediate in Methanol Synthesis by Cu-ZSM-5. J. Am. Chem. Soc. 2010, 132, 14736-14738.

(137) Henson, M. J.; Mukherjee, P.; Root, D. E.; Stack, T. D. P.; Solomon, E. I. Spectroscopic and Electronic Structural Studies of the $\mathrm{Cu}$ (III) 2 Bis- $\mu$-Oxo Core and Its Relation to the Side-on Peroxo-Bridged Dimer. J. Am. Chem. Soc. 1999, $121,10332-10345$.

(138) Mahapatra, S.; Halfen, J. A.; Wilkinson, E. C.; Pan, G.; Cramer, C. J.; Que, L. J.; Tolman, W. B. A New Intermediate in Copper Dioxygen Chemistry: Breaking the OO Bond To Form a \{Cu2 (. Mu.-O) 2\} 2+ Core. J. Am. Chem. Soc. 1995, 117, 8865-8866.

(139) Maiti, D.; Fry, H. C.; Woertink, J. S.; Vance, M. A.; Solomon, E. I.; Karlin, K. D. A 1: 1 Copper- Dioxygen Adduct Is an End-on Bound Superoxo Copper (II) Complex Which Undergoes Oxygenation Reactions with Phenols. J. Am. Chem. Soc. 2007, 129, 264-265.

(140) Chen, P.; Root, D. E.; Campochiaro, C.; Fujisawa, K.; Solomon, E. I. Spectroscopic and Electronic Structure Studies of the Diamagnetic Side-On CullSuperoxo Complex Cu (O2)[HB (3-R-5-I Prpz) 3]: Antiferromagnetic Coupling versus Covalent Delocalization. J. Am. Chem. Soc. 2003, 125, 466-474.

(141) Chen, P.; Fujisawa, K.; Solomon, E. I. Spectroscopic and Theoretical Studies of 
Mononuclear Copper (II) Alkyl-and Hydroperoxo Complexes: Electronic Structure Contributions to Reactivity. J. Am. Chem. Soc. 2000, 122, 10177-10193.

(142) Czernuszewicz, R. S.; Sheats, J. E.; Spiro, T. G. Resonance Raman Spectra and Excitation Profile for Bis (Acetato) Bis (Hydrotripyrazolylborato) Oxodiiron, a Hemerythrin Analog. Inorg. Chem. 1987, 26, 2063-2067.

(143) Mentzen, B. F.; Bergeret, G. Crystallographic Determination of the Positions of the Copper Cations in Zeolite MFI. J. Phys. Chem. C 2007, 111, 12512-12516.

(144) Pieterse, J. A. Z.; Pirngruber, G. D.; van Bokhoven, J. A.; Booneveld, S. Hydrothermal Stability of Fe-ZSM-5 and Fe-BEA Prepared by Wet lon-Exchange for N 2 O Decomposition. Appl. Catal. B Environ. 2007, 71, 16-22.

(145) Nováková, J.; Schwarze, M.; Sobalík, Z. Role of Zeolitic Oxygens During the Decomposition of 15 N. Catal. Lett. 2005, 104, 157-162.

(146) Fu, C. M.; Korchak, V. N.; Hall, W. K. Decomposition of Nitrous Oxide on FeY Zeolite. J. Catal. 1981, 68, 166-171.

(147) Corma, A.; García, H. Lewis Acids as Catalysts in Oxidation Reactions: From Homogeneous to Heterogeneous Systems. Chem. Rev. 2002, 102, 3837-3892.

(148) García, H.; Roth, H. D. Generation and Reactions of Organic Radical Cations in Zeolites. Chem. Rev. 2002, 102, 3947-4008.

(149) Yoon, K. B. Electron-and Charge-Transfer Reactions within Zeolites. Chem. Rev. 1993, 93, 321-339.

(150) Ipek, B.; Wulfers, M. J.; Kim, H.; Göltl, F.; Hermans, I.; Smith, J. P.; Booksh, K. S.; Brown, C. M.; Lobo, R. F. Formation of [Cu2O2]2+ and [Cu2O]2+ toward C-H Bond Activation in Cu-SSZ-13 and Cu-SSZ-39. ACS Catal. 2017, 7, 4291-4303. 
(151) Johnston, E. M.; Carreira, C.; Dell'Acqua, S.; Dey, S. G.; Pauleta, S. R.; Moura, I.; Solomon, E. I. Spectroscopic Definition of the CuZ ${ }^{\circ}$ Intermediate in Turnover of Nitrous Oxide Reductase and Molecular Insight into the Catalytic Mechanism. J. Am. Chem. Soc. 2017, 139, 4462-4476.

(152) Ipek, B.; Lobo, R. F. Catalytic Conversion of Methane to Methanol on Cu-SSZ-13 Using N2O as Oxidant. Chem. Commun. 2016, 52, 13401-13404.

(153) Sheppard, T.; Daly, H.; Goguet, A.; Thompson, J. M. Improved Efficiency for Partial Oxidation of Methane by Controlled Copper Deposition on SurfaceModified ZSM-5. ChemCatChem 2016, 8, 562-570.

(154) Narsimhan, K.; lyoki, K.; Dinh, K.; Román-Leshkov, Y. Catalytic Oxidation of Methane into Methanol over Copper-Exchanged Zeolites with Oxygen at Low Temperature. ACS Cent. Sci. 2016, 2, 424-429.

(155) Tomkins, P.; Mansouri, A.; Bozbag, S. E.; Krumeich, F.; Park, M. B.; Alayon, E. M. C.; Ranocchiari, M.; van Bokhoven, J. A. Isothermal Cyclic Conversion of Methane into Methanol over Copper-Exchanged Zeolite at Low Temperature. Angew. Chemie Int. Ed. 2016, 55, 5467-5471.

(156) Sushkevich, V. L.; Palagin, D.; Ranocchiari, M.; van Bokhoven, J. A. Selective Anaerobic Oxidation of Methane Enables Direct Synthesis of Methanol. Science 2017, 356, 523-527.

(157) Tomkins, P.; Ranocchiari, M.; van Bokhoven, J. A. Direct Conversion of Methane to Methanol under Mild Conditions over Cu-Zeolites and beyond. Acc. Chem. Res. 2017, 50, 418-425.

(158) Anufrienko, V. F.; Bulgakov, N. N.; Vasenin, N. T.; Yashnik, S. A.; Tsikoza, L. T.; 
Vosel', S. V; Ismagilov, Z. R. Detection of O- Radical Anions in Cu-ZSM-5

Zeolites after Heat Treatment. Dokl. Chem. 2002, 386, 273-276.

(159) Yashnik, S. A.; Ismagilov, Z. R.; Anufrienko, V. F. Catalytic Properties and Electronic Structure of Copper lons in Cu-ZSM-5. Catal. Today 2005, 110, 310322.

(160) Himes, R. A.; Barnese, K.; Karlin, K. D. One Is Lonely and Three Is a Crowd: Two Coppers Are for Methane Oxidation. Angew. Chemie Int. Ed. 2010, 49, 67146716.

(161) Lieberman, R. L.; Rosenzweig, A. C. Crystal Structure of a Membrane-Bound Metalloenzyme That Catalyses the Biological Oxidation of Methane. Nature 2005, $434,177-182$.

(162) Balasubramanian, R.; Smith, S. M.; Rawat, S.; Yatsunyk, L. A.; Stemmler, T. L.; Rosenzweig, A. C. Oxidation of Methane by a Biological Dicopper Centre. Nature 2010, 465, 115-119.

(163) Chan, S. I.; Chen, K. H. C.; Yu, S. S. F.; Chen, C. L.; Kuo, S. S. J. Toward Delineating the Structure and Function of the Particulate Methane Monooxygenase from Methanotrophic Bacteria. Biochemistry 2004, 43, 44214430.

(164) Ng, K.-Y.; Tu, L.-C.; Wang, Y.-S.; Chan, S. I.; Yu, S. S. F. Probing the Hydrophobic Pocket of the Active Site in the Particulate Methane Monooxygenase (pMMO) fromMethylococcus Capsulatus (Bath) by Variable Stereoselective Alkane Hydroxylation and Olefin Epoxidation. ChemBioChem 2008, 9, 11161123. 
(165) Chan, S. I.; Wang, V. C. C.; Lai, J. C. H.; Yu, S. S. F.; Chen, P. P. Y.; Chen, K. H. C.; Chen, C.-L.; Chan, M. K. Redox Potentiometry Studies of Particulate Methane Monooxygenase: Support for a Trinuclear Copper Cluster Active Site. Angew. Chemie Int. Ed. 2007, 46, 1992-1994.

(166) Chan, S. I.; Lu, Y.-J.; Nagababu, P.; Maji, S.; Hung, M.-C.; Lee, M. M.; Hsu, I.-J.; Minh, P. D.; Lai, J. C.-H.; Ng, K. Y.; et al. Efficient Oxidation of Methane to Methanol by Dioxygen Mediated by Tricopper Clusters. Angew. Chemie Int. Ed. 2013, 52, 3731-3735.

(167) Fierro, G.; Ferraris, G.; Moretti, G. CuO Nanoparticles Entrapped in MFI Framework: Investigation of Textural, Magnetic and Catalytic Properties of CuZSM-5 and Cu-S-1 Catalysts. Appl. Catal. B Environ. 2009, 91, 499-506.

(168) Verma, A. A.; Bates, S. A.; Anggara, T.; Paolucci, C.; Parekh, A. A.; Kamasamudram, K.; Yezerets, A.; Miller, J. T.; Delgass, W. N.; Schneider, W. F.; et al. NO Oxidation: A Probe Reaction on Cu-SSZ-13. J. Catal. 2014, 312, 179190.

(169) Roudesly, F.; Oble, J.; Poli, G. Metal-Catalyzed CH Activation/functionalization: The Fundamentals. J. Mol. Catal. A Chem. 2017, 426, 275-296.

(170) Groves, J. T. Key Elements of the Chemistry of Cytochrome P-450: The Oxygen Rebound Mechanism. J. Chem. Educ. 1985, 62, 928.

(171) Mahyuddin, M. H.; Staykov, A.; Shiota, Y.; Miyanishi, M.; Yoshizawa, K. Roles of Zeolite Confinement and $\mathrm{Cu}-\mathrm{O}-\mathrm{Cu}$ Angle on the Direct Conversion of Methane to Methanol by [Cu2( $\mu-O)] 2+-E x c h a n g e d ~ A E I, C H A, A F X$, and MFI Zeolites. ACS Catal. 2017, 7, 3741-3751. 
(172) Varghese, J. J.; Trinh, Q. T.; Mushrif, S. H. Insights into the Synergistic Role of Metal-lattice Oxygen Site Pairs in Four-Centered C-H Bond Activation of Methane: The Case of CuO. Catal. Sci. Technol. 2016, 6, 3984-3996.

(173) Alayon, E. M.; Nachtegaal, M.; Ranocchiari, M.; van Bokhoven, J. A. Catalytic Conversion of Methane to Methanol over Cu-mordenite. Chem. Commun. 2012, 48, 404-406.

(174) Donoghue, P. J.; Tehranchi, J.; Cramer, C. J.; Sarangi, R.; Solomon, E. I.; Tolman, W. B. Rapid C-H Bond Activation by a Monocopper(III)-Hydroxide Complex. J. Am. Chem. Soc. 2011, 133, 17602-17605.

(175) Yin, G.; Danby, Andrew, M.; Kitko, D.; Carter, J. D.; Scheper, W. M.; Busch, D. H. Understanding the Selectivity of a Moderate Oxidation Catalyst: Hydrogen Abstraction by a Fully Characterized, Activated Catalyst, the Robust Dihydroxo Manganese(IV) Complex of a Bridged Cyclam. J. Am. Chem. Soc. 2007, 129, 1512-1513.

(176) Yin, G.; Danby, A. M.; Kitko, D.; Carter, J. D.; Scheper, W. M.; Busch, D. H. Oxidative Reactivity Difference among the Metal Oxo and Metal Hydroxo Moieties: pH Dependent Hydrogen Abstraction by a Manganese(IV) Complex Having Two Hydroxide Ligands. J. Am. Chem. Soc. 2008, 130, 16245-16253.

(177) Rezabal, E.; Ruipérez, F.; Ugalde, J. M. Quantum Chemical Study of the Catalytic Activation of Methane by Copper Oxide and Copper Hydroxide Cations. Phys. Chem. Chem. Phys. 2013, 15, 1148-1153.

(178) Rosa, A.; Ricciardi, G.; Baerends, E. J. Is [FeO] 2+ the Active Center Also in Iron Containing Zeolites? A Density Functional Theory Study of Methane 
Hydroxylation Catalysis by Fe-ZSM-5 Zeolite. Inorg. Chem. 2010, 49, 3866-3880.

(179) Dubkov, K. A.; Sobolev, V. I.; Panov, G. I. Low-Temperature Oxidation of Methane to Methanol on FeZSM-5 Zeolite. Kinet. Catal. 1998, 39, 72-79.

(180) Ovanesyan, N. S.; Shteinman, A. A.; Dubkov, K. A.; Sobolev, V. I.; Panov, G. I. The State of Iron in the Fe-ZSM-5-N2O System for Selective Oxidation of Methane to Methanol from Data of Mössbauer Spectroscopy. Kinet. Catal. 1998, 39, 792-797.

(181) Panov, G. I. Advances in Oxidation Catalysis; Oxidation of Benzene to Phenol by Nutrous Oxide. CATTECH 2000, 4, 18-31.

(182) Centi, G.; Genovese, C.; Giordano, G.; Katovic, A.; Perathoner, S. Performance of Fe-BEA Catalysts for the Selective Hydroxylation of Benzene with N2O. Catal. Today. 2004, 91-92, 17-26.

(183) Smeets, P. J.; Woertink, J. S.; Sels, B. F.; Solomon, E. I.; Schoonheydt, R. A. Transition-Metal Ions in Zeolites: Coordination and Activation of Oxygen. Inorg. Chem. 2010, 49, 3673-3583.

(184) Hammond, C.; Dimitratos, N.; Jenkins, R. L.; Lopez-sanchez, J. A.; Kondrat, S. A.; Hasbi, M.; Forde, M. M.; Thetford, A.; Taylor, S. H.; Hagen, H.; et al. Elucidation and Evolution of the Active Component within Cu/Fe/ ZSM - 5 for Catalytic Methane Oxidation: From Synthesis to Catalysis. ACS Catal. 2013, 3, 689-699.

(185) Baerlocher, C.; McCusker, L. B.; Olson, D. H. Atlas of Zeolite Framework Types; Elsevier: Amsterdam, 2007.

(186) Newsam, J. M.; Treacy, M. M.; Koetsier, W. T.; De Gruyter, C. B. Structural 
Characterization of Zeolite Beta. Proc. R. Soc. London A Math. Phys. Eng. Sci. 1988, 420, 375-405.

(187) Vaughan, P. A. The Crystal Structure of the Zeolite Ferrierite. Acta Crystallogr. 1966, 21, 983-990.

(188) Kokotailo, G. T.; Lawton, S. L.; Olson, D. H. Structure of Synthetic Zeolite ZSM-5. Nature 1978, 272, 437-438.

(189) Sklenak, S.; Andrikopoulos, P. C.; Boekfa, B.; Jansang, B.; Nováková, J.; Benco, L.; Bucko, T.; Hafner, J.; Dědeček, J.; Sobalík, Z. \{N2O\} Decomposition over FeZeolites: Structure of the Active Sites and the Origin of the Distinct Reactivity of Fe-Ferrierite, Fe-ZSM-5, and Fe-Beta. A Combined Periodic $\{$ DFT $\}$ and Multispectral Study. J. Catal. 2010, 272, 262-274.

(190) Burns, R. G.; Clark, M. G.; Stone, A. J. Vibronic Polarization in the Electronic Spectra of Gillespite, a Mineral Containing Iron (II) in Square-Planar Coordination. Inorg. Chem. 1966, 5, 1268-1272.

(191) Cantalupo, S. A.; Fiedler, S. R.; Shores, M. P.; Rheingold, A. L.; Doerrer, L. H. High- Spin Square- Planar Coll and Fell Complexes and Reasons for Their Electronic Structure. Angew. Chemie Int. Ed. 2012, 51, 1000-1005.

(192) Pinkert, D.; Demeshko, S.; Schax, F.; Braun, B.; Meyer, F.; Limberg, C. A Dinuclear Molecular Iron (II) Silicate with Two High- Spin Square- Planar FeO4 Units. Angew. Chemie Int. Ed. 2013, 52, 5155-5158.

(193) Pascualini, M. E.; Di Russo, N. V; Thuijs, A. E.; Ozarowski, A.; Stoian, S. A.; Abboud, K. A.; Christou, G.; Veige, A. S. A High-Spin Square-Planar Fe(II) Complex Stabilized by a Trianionic Pincer-Type Ligand and Conclusive Evidence 
for Retention of Geometry and Spin State in Solution.

(194) Clark, M. G.; Bancroft, G. M.; Stone, A. J. Mössbauer Spectrum of Fe2+ in a Square- Planar Environment. J. Chem. Phys. 1967, 47, 4250-4261.

(195) Wurzenberger, X.; Piotrowski, H.; Klüfers, P. A Stable Molecular Entity Derived from Rare Iron (II) Minerals: The Square- Planar High- Spin- d6 FellO4 Chromophore. Angew. Chemie Int. Ed. 2011, 50, 4974-4978.

(196) Neidig, M. L.; Decker, A.; Choroba, O. W.; Huang, F.; Kavana, M.; Moran, G. R.; Spencer, J. B.; Solomon, E. I. Spectroscopic and Electronic Structure Studies of Aromatic Electrophilic Attack and Hydrogen-Atom Abstraction by Non-Heme Iron Enzymes. Proc. Natl. Acad. Sci. 2006, 103, 12966-12973.

(197) Rossi, A. R.; Hoffmann, R. Transition Metal Pentacoordination. Inorg. Chem. $1975,14,365-374$.

(198) Srnec, M.; Wong, S. D.; Matthews, M. L.; Krebs, C.; Bollinger, J. M.; Solomon, E. I. Electronic Structure of the Ferryl Intermediate in the $\alpha$-Ketoglutarate Dependent Non-Heme Iron Halogenase SyrB2: Contributions to H Atom Abstraction Reactivity. J. Am. Chem. Soc. 2016, 138, 5110.

(199) Srnec, M.; Wong, S. D.; England, J.; Que, L.; Solomon, E. I. T-Frontier Molecular Orbitals in $\mathrm{S}=2$ Ferryl Species and Elucidation of Their Contributions to Reactivity. Proc. Natl. Acad. Sci. 2012, 109, 14326-14331.

(200) McDonald, A. R.; Que, L. High-Valent Nonheme Iron-Oxo Complexes: Synthesis, Structure, and Spectroscopy. Coord. Chem. Rev. 2013, 257, 414-428.

(201) Starokon, E. V; Parfenov, M. V; Arzumanov, S. S.; Pirutko, L. V; Stepanov, A. G.; Panov, G. I. Oxidation of Methane to Methanol on the Surface of FeZSM-5 
Zeolite. J. Catal. 2013, 300, 47-54.

(202) Panov, G. I.; Dubkov, K. A.; Paukshtis, Y. A. Identification of Active Oxygen Species over Fe Complexes in Zeolites. In Catalysis by Unique Metal Ion Structures in Solid Matrices: From Science to Application; Centi, G., Wichterlová, B., Bell, A. T., Eds.; Springer Netherlands: Dordrecht, 2001; pp 149-163.

(203) de Visser, S. P.; Oh, K.; Han, A.-R.; Nam, W. Combined Experimental and Theoretical Study on Aromatic Hydroxylation by Mononuclear Nonheme Iron (IV)Oxo Complexes. Inorg. Chem. 2007, 46, 4632-4641.

(204) Hammond, C.; Jenkins, R. L.; Dimitratos, N.; Lopez-sanchez, J. A.; Forde, M. M.; Thetford, A.; Murphy, D. M.; Hagen, H.; Stangland, E. E.; Moulijn, J. M.; et al. Catalytic and Mechanistic Insights of the Low-Temperature Selective Oxidation of Methane over Cu-Promoted Fe-ZSM-5. Chem. - A Eur. J. 2012, 18, 1573515745.

(205) Hammond, C.; Dimitratos, N.; Lopez-Sanchez, J. A.; Jenkins, R. L.; Whiting, G.; Kondrat, S. A.; Ab Rahim, M. H.; Forde, M. M.; Thetford, A.; Hagen, H.; et al. Aqueous-Phase Methane Oxidation over Fe-MFI Zeolites; Promotion through Isomorphous Framework Substitution. ACS Catal. 2013, 3, 1835-1844.

(206) Vanelderen, P.; Hadt, R. G.; Smeets, P. J.; Solomon, E. I.; Schoonheydt, R. A.;

Sels, B. F. Cu-ZSM-5: A Biomimetic Inorganic Model for Methane Oxidation. J. Catal. 2011, 284, 157-164.

(207) Panov, G. I.; Sobolev, V. I.; Dubkov, K. A.; Parmon, V. N.; Ovanesyan, N. S.; Shilov, A. E.; Shteinman, A. A. Iron Complexes in Zeolites as a New Model of Methane Monooxygenase. React. Kinet. Catal. Lett. 1997, 61, 251-258. 
(208) Hanson, R. S.; Hanson, T. E. Methanotrophic Bacteria. Microbiol. Rev. 1996, 60, $439-471$.

(209) Culpepper, M. A.; Cutsail, G. E.; Gunderson, W. A.; Hoffman, B. M.; Rosenzweig, A. C. Identification of the Valence and Coordination Environment of the Particulate Methane Monooxygenase Copper Centers by Advanced EPR Characterization. J. Am. Chem. Soc. 2014, 136, 11767-11775.

(210) Lieberman, R. L.; Kondapalli, K. C.; Shrestha, D. B.; Hakemian, A. S.; Smith, S. M.; Telser, J.; Kuzelka, J.; Gupta, R.; Borovik, A. S.; Lippard, S. J.; et al. Characterization of the Particulate Methane Monooxygenase Metal Centers in Multiple Redox States by X-Ray Absorption Spectroscopy. Inorg. Chem. 2006, 45, 8372-8381.

(211) Culpepper, M. A.; Cutsail, G. E.; Hoffman, B. M.; Rosenzweig, A. C. Evidence for Oxygen Binding at the Active Site of Particulate Methane Monooxygenase. J. Am. Chem. Soc. 2012, 134, 7640-7643.

(212) Solomon, E. I.; Ginsbach, J. W.; Heppner, D. E.; Kieber-Emmons, M. T.;

Kjaergaard, C. H.; Smeets, P. J.; Tian, L.; Woertink, J. S. Copper Dioxygen (Bio)inorganic Chemistry. Faraday Discuss. 2011, 148, 11-39.

(213) Matoba, Y.; Kumagai, T.; Yamamoto, A.; Yoshitsu, H.; Sugiyama, M. Crystallographic Evidence That the Dinuclear Copper Center of Tyrosinase Is Flexible during Catalysis. J. Biol. Chem. 2006, 281, 8981-8990.

(214) Eickman, N. C.; Solomon, E. I.; Larrabee, J. A.; Spiro, T. G.; Lerch, K. Ultraviolet Resonance Raman Study of Oxytyrosinase. Comparison with Oxyhemocyanins. J. Am. Chem. Soc. 1978, 100, 6529-6531. 
(215) Henson, M. J.; Mahadevan, V.; Stack, T. D. P.; Solomon, E. I. A New Cu (II) Sideon Peroxo Model Clarifies the Assignment of the Oxyhemocyanin Raman Spectrum. Inorg. Chem. 2001, 40, 5068-5069.

(216) Matsubara, T.; Frunzke, K.; Zumft, W. G. Modulation by Copper of the Products of Nitrite Respiration in Pseudomonas Perfectomarinus. J. Bacteriol. 1982, 149, 816-823.

(217) Brown, K.; Djinovic-Carugo, K.; Haltia, T.; Cabrito, I.; Saraste, M.; Moura, J. G.; Moura, I.; Tegoni, M.; Cambillau, C. Revisiting the Catalytic CuZ Cluster of Nitrous Oxide (N2O) Reductase Evidence of a Bridging Inorganic Sulfur. J. Biol. Chem. 2000, 275, 41133-41136.

(218) Brown, K.; Tegoni, M.; Prudêncio, M.; Pereira, A. S.; Besson, S.; Moura, J. J.; Moura, I.; Cambillau, C. A Novel Type of Catalytic Copper Cluster in Nitrous Oxide Reductase. Nat. Struct. Mol. Biol. 2000, 7, 191-195.

(219) Chen, P.; Cabrito, I.; Moura, J. J. G.; Moura, I.; Solomon, E. I. Spectroscopic and Electronic Structure Studies of the $\mu 4$-Sulfide Bridged Tetranuclear CuZ Cluster in N2O Reductase: Molecular Insight into the Catalytic Mechanism. J. Am. Chem. Soc. 2002, 124, 10497-10507.

(220) Rosenzweig, A. C.; Nordlund, P.; Takahara, P. M.; Frederick, C. A.; Lippard, S. J. Geometry of the Soluble Methane Monooxygenase Catalytic Diiron Center in Two Oxidation States. Chem. Biol. 1995, 2, 409-418.

(221) Lee, S.-K.; Lipscomb, J. D. Oxygen Activation Catalyzed by Methane Monooxygenase Hydroxylase Component: Proton Delivery during the O- O Bond Cleavage Steps. Biochemistry 1999, 38, 4423-4432. 
(222) Shu, L.; Nesheim, J. C.; Kauffmann, K.; Münck, E.; Lipscomb, J. D.; Que, L. An Fe2IVO2 Diamond Core Structure for the Key Intermediate $Q$ of Methane Monooxygenase. Science. 1997, 275, 515-518.

(223) Banerjee, R.; Proshlyakov, Y.; Lipscomb, J. D.; Proshlyakov, D. A. Structure of the Key Species in the Enzymatic Oxidation of Methane to Methanol. Nature 2015, 518, 431-434.

(224) Ambundo, E. A.; Friesner, R. A.; Lippard, S. J. Reactions of Methane Monooxygenase Intermediate $Q$ with Derivatized Methanes. J. Am. Chem. Soc. 2002, 124, 8770-8771.

(225) Baik, M.-H.; Gherman, B. F.; Friesner, R. A.; Lippard, S. J. Hydroxylation of Methane by Non-Heme Diiron Enzymes: Molecular Orbital Analysis of C- H Bond Activation by Reactive Intermediate Q. J. Am. Chem. Soc. 2002, 124, 1460814615.

(226) Choroba, O. W.; Williams, D. H.; Spencer, J. B. Biosynthesis of the Vancomycin Group of Antibiotics: Involvement of an Unusual Dioxygenase in the Pathway to (S)-4-Hydroxyphenylglycine. J. Am. Chem. Soc. 2000, 122, 5389-5390.

(227) Johnson-Winters, K.; Purpero, V. M.; Kavana, M.; Nelson, T.; Moran, G. R. (4Hydroxyphenyl) Pyruvate Dioxygenase from Streptomyces Avermitilis: The Basis for Ordered Substrate Addition. Biochemistry 2003, 42, 2072-2080.

(228) Fritze, I. M.; Linden, L.; Freigang, J.; Auerbach, G.; Huber, R.; Steinbacher, S. The Crystal Structures of Zea Mays and Arabidopsis 4-Hydroxyphenylpyruvate Dioxygenase. Plant Physiol. 2004, 134, 1388-1400.

(229) Koehntop, K. D.; Emerson, J. P.; Que, L. The 2-His-1-Carboxylate Facial Triad: A 
Versatile Platform for Dioxygen Activation by Mononuclear Non-Heme iron(II) Enzymes. J. Biol. Inorg. Chem. 2005, 10, 87-93.

(230) Neidig, M. L.; Kavana, M.; Moran, G. R.; Solomon, E. I. CD and MCD Studies of the Non-Heme Ferrous Active Site in (4-Hydroxyphenyl) Pyruvate Dioxygenase: Correlation between Oxygen Activation in the Extradiol and a-KG-Dependent Dioxygenases. J. Am. Chem. Soc. 2004, 126, 4486-4487.

(231) Davies, G.; El-Sayed, M. A. Stoichiometry and Kinetics of the Oxidation of Halo (Pyridine) Copper (I) Complexes by Dioxygen in Aprotic Solvents. Effects of Copper (I) Reactant Molecularity on the Rate Law and Evidence for LigandDependent Product Structures. Inorg. Chem. 1983, 22, 1257-1266.

(232) Lapinte, C.; Riviere, H.; Roselli, A.; Fabre, C. Role of a Copper Complex in an Oxygen-Atom Transfer Reaction from Dioxygen to Triphenylphosphine. J. Chem. Soc. Chem. Commun. 1981, No. 21, 1109-1110.

(233) Karlin, K. D.; Gultneh, Y.; Hayes, J. C.; Zubieta, J. Copper (I)-Dioxygen Reactivity. II: Reaction of a Three-Coordinate Copper (I) Complex with O2, with Evidence for a Binuclear Oxo-Copper (II) Species: Structural Characterization of a ParallelPlanar Dihydroxo-Bridged Dimer. Inorg. Chem. 1984, 23, 519-521.

(234) Kitajima, N.; Koda, T.; Hashimoto, S.; Kitagawa, T.; Morooka, Y. Synthesis and Characterization of the Dinuclear copper(II) Complexes $[\mathrm{Cu}(\mathrm{HB}(3,5-\mathrm{Me} 2 \mathrm{pz}) 3)] 2 \mathrm{X}$ (X = O2-, (OH)22-, CO22-, O22-). J. Am. Chem. Soc. 1991, 113, 5664-5671.

(235) Haack, P.; Kärgel, A.; Greco, C.; Dokic, J.; Braun, B.; Pfaff, F. F.; Mebs, S.; Ray, K.; Limberg, C. Access to a Cull-O-Cull Motif: Spectroscopic Properties, Solution Structure, and Reactivity. J. Am. Chem. Soc. 2013, 135, 16148-16160. 
(236) Csicsery, S. M. Shape-Selective Catalysis in Zeolites. Zeolites 1984, 4, 202-213.

(237) Kroll, T.; Hadt, R. G.; Wilson, S. A.; Lundberg, M.; Yan, J. J.; Weng, T.-C.;

Sokaras, D.; Alonso-Mori, R.; Casa, D.; Upton, M. H. Resonant Inelastic X-Ray

Scattering on Ferrous and Ferric Bis-Imidazole Porphyrin and Cytochrome c:

Nature and Role of the Axial Methionine-Fe Bond. J. Am. Chem. Soc. 2014, 136, 18087-18099.

(238) Yoon, J.; Fujii, S.; Solomon, E. I. Geometric and Electronic Structure Differences between the Type 3 Copper Sites of the Multicopper Oxidases and Hemocyanin/tyrosinase. Proc. Natl. Acad. Sci. 2009, 106, 6585-6590.

(239) Traa, Y.; Burger, B.; Weitkamp, J. Zeolite-Based Materials for the Selective Catalytic Reduction of NOx with Hydrocarbons. Microporous Mesoporous Mater. 1999, 30, 3-41.

(240) Sutherlin, K. D.; Liu, L. V; Lee, Y.-M.; Kwak, Y.; Yoda, Y.; Saito, M.; Kurokuzu, M.; Kobayashi, Y.; Seto, M.; Que, L.; et al. Nuclear Resonance Vibrational Spectroscopic Definition of Peroxy Intermediates in Nonheme Iron Sites. J. Am. Chem. Soc. 2016, 138, 14294-14302. 


\section{Author Biographies}

Benjamin E.R. Snyder received his B.S. in chemistry and B.A. in mathematics from the University of Rochester in 2012. He is now an NSF predoctoral fellow and Stanford Graduate Fellow pursuing his Ph.D. in physical inorganic chemistry at Stanford University under the supervision of Prof. Edward Solomon. His research focuses on spectroscopic and computational elucidation of $\mathrm{Cu}$ and Fe active sites in zeolites, their activation for selective hydrocarbon oxidation, and their correspondence to $\mathrm{Cu}$ and $\mathrm{Fe}$ active sites in biology.

Max L. Bols received his B.S. and M.S. in bioscience engineering at the KU Leuven in 2015, doing his Master's thesis on the identification of the active site in iron-zeolites for methane activation. Upon graduation, he started a Ph.D. with prof. Bert Sels on the synthesis, kinetics and spectroscopic characterization of transition metal active sites in zeolites, in the framework of an FWO research grant.

Robert A. Schoonheydt is emeritus professor of the KU Leuven, where he obtained his bioengineering degree in 1966 and his Ph.D. in 1970 under the guidance of prof. Jan B. Uytterhoeven. He did a postdoc in the chemistry department of the Texas A\&M University under guidance of Jack $\mathrm{H}$. Lunsford on adsorption of $\mathrm{SO}_{2}$ on $\mathrm{MgO}$. His research interests at the $\mathrm{KU}$ Leuven are the surface chemistry of clay minerals and zeolites with special emphasis on transition metal ions and spectroscopy. Presently he is a member of an international research collaboration of zeolite chemists, 
spectroscopists and quantum chemists on active sites in $\mathrm{Cu}$ - and Fe-zeolites for selective oxidation, $\mathrm{NO}_{x}$ abatement and electrophilic aromatic substitution reactions. He has been dean of the faculty of bioscience engineering from 1998 till 2004 and president of the International Association for the Study of Clays from 2001 till 2005.

Bert F. Sels (1972), currently full professor at KU Leuven (Belgium), obtained his Ph.D. in 2000 in the field of heterogeneous oxidation catalysis under guidance of professor Pierre Jacobs. He was awarded the DSM Chemistry Award in 2000, the Incentive Award by the Belgian Chemical Society in 2005, and the international Green Chemistry Award in 2015. He is currently director of the Centre for Surface Chemistry and Catalysis (COK), and active in designing heterogeneous catalysts for future challenges in industrial organic and environmental catalysis. His expertise includes heterogeneous catalysis in bio-refineries, design of hierarchical zeolites and carbons, and the spectroscopic and kinetic study of active sites for small-molecule activation. He is cochair of the Catalysis Commission of the International Zeolite Association (IZA) and cofounder of European Research Institute of Catalysis (ERIC). He is also member of the European Academy of Sciences and Arts, member of the international advisory board of ChemSusChem (Wiley) and associate editor of ACS Sustainable Chemistry \& Engineering.

Edward I. Solomon Edward I. Solomon grew up in North Miami Beach, FL, received his Ph.D. at Princeton (with D. S. McClure), and was a postdoctoral fellow at The Ørsted Institute (with C. J. Ballhausen) and then at Caltech (with H. B. Gray). He was a 
professor at the Massachusetts Institute of Technology until 1982, when he joined the faculty at Stanford University, where he is now the Monroe E. Spaght Professor of Humanities and Sciences and Professor of Photon Science at the SLAC National Accelerator Lab. He has been an invited professor in Argentina, Australia, Brazil, China, France, India, and Japan. Prof. Solomon's research is in the fields of physicalinorganic chemistry and bioinorganic chemistry with emphasis on the application of a wide range of spectroscopic methods combined with QM calculations to elucidate the electronic structure of transition-metal sites and its contribution to physical properties and reactivity. He has received a wide range of medals and awards and is a member of the National Academy of Sciences and the American Academy of Arts and Sciences and a fellow in the American Association for the Advancement of Science and the American Chemical Society. 